\title{
Low-Dimensional Physics of Organic-Inorganic Multilayers
}

by

Bridget Ingham

A thesis

submitted to the Victoria University of Wellington in fulfilment of the requirements for the degree of Doctor of Philosophy in Physics

Victoria University of Wellington

August 2005 


\begin{abstract}
This thesis demonstrates the rich low-dimensional physics associated with the class of organic-inorganic hybrid materials based on atomic layers of a metal oxide separated by organic spacer molecules. Hybrid materials based on tungsten oxide and also transition metal tungstates (with manganese, iron, cobalt, nickel and copper) were synthesised and characterised using a variety of techniques. The materials in question represent one example of the huge variety of systems classed as 'organic-inorganic hybrids' and have the potential to combine the high-electron mobility of the metal oxide layers with the propensity for self-assembly of the organic layers.

The crystal structures of the compounds were investigated using powder X-ray diffraction and electron diffraction, and compared with structural information obtained using IR, Raman, and extended X-ray absorption fine structure (EXAFS) spectroscopies. This data confirmed the presence of a 2dimensional layered structure. The electronic properties of the hybrids were studied using optical spectroscopy and confirmed via $a b$ initio calculations. The band gaps of the tungsten oxide hybrids were found to be independent of interlayer spacing, and in all cases were larger than that observed in the three dimensional $\mathrm{WO}_{3}$ 'parent' material. For the transition metal tungstate hybrids there appeared to be significant interactions between the organic amines and the transition metal ions within the inorganic layers.

The magnetic properties of the hybrids incorporating transition metal ions were also studied in detail. Many of these metal tungstate hybrids display magnetic transitions at low temperatures indicating a crossover from 2-dimensional to 3-dimensional behaviour. This illustrates the importance of the low-dimensional nature of the inorganic layers in these hybrid materials
\end{abstract}


and thus their potential in nano-structural applications. 


\section{Foreword}

"Do you not know? Have you not heard? The LORD is the everlasting God, the Creator of the ends of the earth. He will not grow tired or weary, and His understanding no-one can fathom. He gives strength to the weary and increases the power of the weak. Even youths grow tired and weary, and young men stumble and fall; but those who hope in the LORD will renew their strength. They will soar on wings like eagles; they will run and not grow weary, they will walk and not be faint." - Isaiah 40:28-31

This work forms the written component of the research undertaken in the period January 2002 to February 2005, to fulfill the requirements of the Victoria University of Wellington PhD degree. The work presented in this thesis has also been published in the following publications. Others are in preparation or are planned.

1. B. Ingham, S.V. Chong and J.L. Tallon, 'Novel Materials Based on Organic-Tungsten Oxide Hybrid Systems' in Self-Assembled Nanostructured Materials, Materials Research Society Symposia Proceedings 775 (2003) p. 165-168.

2. S.V. Chong, B. Ingham and J.L. Tallon, 'Novel Materials Based on Organic-Tungsten Oxide Hybrid Systems: I. Synthesis and Characterisation', Current Applied Physics 4 (2004) p. 197-201.

3. B. Ingham, S.V. Chong and J.L. Tallon, 'Novel Materials Based on Organic-Tungsten Oxide Hybrid Systems: II. Electronic Properties of the W-O Framework', Current Applied Physics 4 (2004) p. 202-205. 
4. B. Ingham, S.V. Chong and J.L. Tallon, 'Layered Tungsten OxideBased Hybrid Materials Incorporating Transition Metal Ions', in Organic/Inorganic Hybrid Materials, Materials Research Society Symposia Proceedings 847 (2005) EE9.31.

5. S.V. Chong, B. Ingham and J.L. Tallon, 'Two Synthetic Routes to the Formation of Tungsten Oxide Hybrid Compounds', in Organic/Inorganic Hybrid Materials, Materials Research Society Symposia Proceedings, 847 (2005) EE13.14.

6. B. Ingham, S.V. Chong and J.L. Tallon, 'Layered Tungsten Oxide-Based Organic-Inorganic Hybrid Materials: An Infrared and Raman Study', Journal of Physical Chemistry B 109 (2005) p. 4936-4940.

7. B. Ingham, S.V. Chong and J.L. Tallon, 'Organic-Inorganic Layered Hybrid Materials', Invited chapter contribution to 'Soft Condensed Matter: New Research', Nova Publishers (accepted for publication).

8. B. Ingham, S.C. Hendy, S.V. Chong and J.L. Tallon, 'Density functional studies of tungsten trioxide, tungsten bronzes, and related systems', Physical Review B (accepted for publication).

Results herein have been presented on the following occasions:

1. Invited seminars given at:

(a) Industrial Research Limited, Lower Hutt, New Zealand (2004).

(b) Cambridge University, Cambridge, United Kingdom (August 2004).

(c) Max Planck Institut für Festkörperforschung (Max Planck Institute for Solid State Research), Stuttgart, Germany (August 2004).

2. Contributed presentations given at:

(a) Advanced Materials and Nanotechnology, 2nd International Conference (AMN-2), Queenstown, New Zealand (February 2005, oral presentation). 
(b) Materials Research Society Fall Meeting, Boston, Massachusetts, USA (November 2004, poster presentation).

(c) Wilhelm und Else Heraeus Summer School, Lutherstadt-Wittenberg, Germany (August 2004, poster presentation).

(d) New Zealand Institute of Physics, 11th National Conference, Palmerston North, New Zealand (July 2003, oral presentation).

(e) Advanced Materials and Nanotechnology, 1st International Conference (AMN-1), Wellington, New Zealand (February 2003, oral presentation). 


\section{Conventions and Abbreviations used}

Abbreviations have been used extensively throughout this thesis to represent the chemical composition of the organic-inorganic hybrid samples. Table 1 illustrates the notation used to represent the organic part.

The most common organic molecules used are the bidentate diaminoalkanes, DAn. The monodentate counterparts have therefore been labelled MAn.

When referring to the hybrid material, the metal ion(s) present are written first, for example, W-DA6 and $(\mathrm{Cu}, \mathrm{W})$-phen refer to tungsten oxide diaminohexane and copper tungstate phenethylamine respectively. It is implicitly assumed that the metal ion is present as an oxide species.

Many different experimental techniques have been used to characterise the hybrid materials and measure their properties. While these are given along with their abbreviation in the text, it is helpful to include a reference table here (Table 2).

SI units have been used throughout, although in the sections on magnetism the odd CGS unit may creep in as a result of using the raw data (e.g. applied field given in Oe $\left(10^{4}\right.$ Oersted $\equiv 1$ Tesla $)$, raw data magnetisation as $\mathrm{emu})$.

\begin{tabular}{|l|l|l|}
\hline Abbreviation & Name & Chemical formula \\
\hline MAn $(n=1,2, \ldots)$ & methyl/ethyl/...-amine & $\mathrm{H}_{2} \mathrm{~N}\left(\mathrm{CH}_{2}\right)_{n-1} \mathrm{CH}_{3}$ \\
DAn $\left(n=2,4, \ldots{ }^{1}\right)$ & diamino-eth/but/...-ane & $\mathrm{H}_{2} \mathrm{~N}\left(\mathrm{CH}_{2}\right)_{n} \mathrm{NH}_{2}$ \\
phen & phenethylamine & $\left(\mathrm{C}_{6} \mathrm{H}_{5}\right)\left(\mathrm{CH}_{2}\right)_{2} \mathrm{NH}_{2}$ \\
DAphen & phenylenediamine & $\mathrm{H}_{2} \mathrm{~N}\left(\mathrm{C}_{6} \mathrm{H}_{4}\right) \mathrm{NH}_{2}$ \\
\hline
\end{tabular}

Table 1: Abbreviations of organic molecules used throughout this thesis.

${ }^{1} \mathrm{n}=$ even only. 


\begin{tabular}{ll}
\hline Abbreviation & Full name \\
\hline \hline EDX & Energy Dispersive X-ray analysis \\
EXAFS & Extended X-ray Absorption Fine Structure \\
IR & Infrared \\
SAED & Selected Area Electron Diffraction \\
SEM & Scanning Electron Microscopy \\
SQUID & Superconducting QUantum Interference Device \\
TEM & Transmission Electron Microscopy \\
TGA & Thermo-gravimetric analysis \\
UV & Ultraviolet \\
VASP & Vienna Ab initio Simulation Package \\
XANES & X-ray Absorption Near Edge Spectroscopy \\
XRD & X-ray diffraction \\
\hline
\end{tabular}

Table 2: Abbreviations of experimental techniques used throughout this thesis, listed in alphabetical order. 


\section{Acknowledgements}

There are many people who have helped either me or the project in various measures. This page contains far less than an exhaustive list, but the following people immediately come to mind:

My supervisors Jeff Tallon and Alan Kaiser. Alan, who handled the administrative side of my $\mathrm{PhD}$ and was always enthusiastic and willing to help at a moment's notice. Jeff, whom it has been a pleasure to work for and with during the last five years. Many thanks for the opportunities, past and present, that I have received that have got me to where I am today.

Thanks to Shen Chong, who stuck with the chemistry of the project right through (and who did the electrochemistry work); Hana Robson-Marsden and Natalie Robinson (nee de Frere) who started the work off for us in the first place in 2001.

The following people who helped with various technical matters (listed alphabetically): Tony Bittar for some of the UV-vis interpretation, Kay Card for all the SEM, Dave Cochrane for all the quartz pieces and thin film evaporation, Graeme Gainsford for his immense patience with regards to solving the structure with difficult data, Peter Gilberd for getting me started on the TEM, John Hamlin for running so many UV-vis samples for us, Shaun Hendy for teaching me VASP, Ling-Yun Jang and Ru-Shi Liu for the XANES and EXAFS experiments, Ben Ruck for the UV-vis analysis, Nick Strickland for the IR reflectance work, Richard Tilley for the TEM at Victoria, Joe Trodahl for the Raman experiments and analysis, and Grant Williams for the Squid work.

Thanks for helpful discussions also go to Ashton Partridge, Tony Woolhouse, Neil Ashcroft, and the many researchers at Cambridge and MPI 
Stuttgart whose names I can't remember.

Thanks to the entire HTS team at IRL for a fun 5 years.

To my family who put up with me being a student for so long, they will be glad to know it all paid off!

And lastly and most importantly, my thanks to God for the peace, support and encouragement He has given me over this time, in the good times and bad, through circumstances and sending the right people across my path at the right time. For planning the course of my life so far, from being in the New Zealand Physics Olympiad team, to not getting in the air force so I would stick with physics, to starting as a summer student in 1999 working for Jeff by 'accident' - and for knowing exactly what He has in store for me in the future. To Him be glory forever and ever... amen. May the words of my mouth and the meditation of my heart be acceptable in Your sight, my Jesus, my Lord, my friend, my Saviour. 


\section{Contents}

Foreword $\quad$ v

Acknowledgements $\quad x i$

1 Introduction 1

1.1 Strongly Correlated Electronic Systems . . . . . . . . . . . 2

1.1.1 Basic Theory . . . . . . . . . . . . . . 2

1.1 .2 Examples ................... 4

1.2 Organic-Inorganic Hybrid Materials . . . . . . . . . . . . 10

1.3 Tungsten Oxide Systems . . . . . . . . . . . . . . . . . . 14

1.4 Thesis outline . . . . . . . . . . . . . . . . . . 21

2 Experimental 25

2.1 Synthesis of tungsten oxide-based hybrids . . . . . . . . 25

2.1.1 Solution-based method . . . . . . . . . . . 26

2.1 .2 'Melt method' . . . . . . . . . . . . . . . 26

2.1.3 Chimie douce method . . . . . . . . . . . . 27

2.1.4 Thin film preparation . . . . . . . . . . . . . . 27

2.2 Hybrids with transition metals . . . . . . . . . . . 28

2.3 Preparation of hybrids for electrochemical doping . . . . . . . 30

2.4 Measurement techniques and parameters . . . . . . . . . . 30

3 Composition and Basic Structure 35

3.1 Synthesis . . . . . . . . . . . . . . . . . 35 
3.1.1 Comparison of synthesis methods . . . . . . . . . 35

$3.1 .2 \mathrm{pH}$ dependence . . . . . . . . . . . . . . . 40

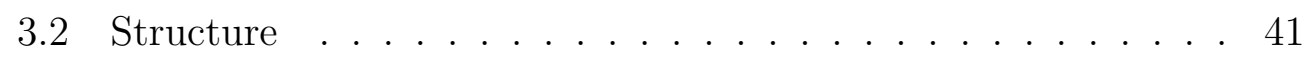

3.2.1 X-ray diffraction . . . . . . . . . . . . . 41

3.2 .2 Electron microscopy . . . . . . . . . . . . . . . . . 44

3.2 .3 Microanalysis . . . . . . . . . . . . . 46

3.2.4 Thermogravimetric analysis . . . . . . . . . 47

3.3 Infrared and Raman spectroscopy . . . . . . . . . . . . . . . 48

3.3.1 $\mathrm{WO}_{3}, \mathrm{H}_{2} \mathrm{WO}_{4}$ and W-DAn samples . . . . . . . 48

3.3 .2 W-MAn samples . . . . . . . . . . . 56

3.3.3 Summary . . . . . . . . . . . . . . . 60

3.4 Structural and composition summary . . . . . . . . . . 60

4 Ab Initio Computations $\quad 63$

4.1 Introduction to technique . . . . . . . . . . . . 64

4.1.1 Computational details . . . . . . . . . . 67

4.2 Previous studies . . . . . . . . . . . . . . . . . . 68

4.3 Cubic and hexagonal alkali bronzes . . . . . . . . . . . 69

4.3 .1 Structure ..................... 69

4.3 .2 Charge-density . . . . . . . . . . . . . 72

4.3.3 Energies of formation . . . . . . . . . . . . . 72

4.3.4 Density of states . . . . . . . . . . . . . 75

4.4 Cubic Sodium Tungsten Bronze Series . . . . . . . . . . . 79

4.5 Sub-stoichiometric $\mathrm{WO}_{3-x} \ldots \ldots \ldots$. . . . . . . . . 81

4.6 Organic-Inorganic Layered Hybrids . . . . . . . . . . . . . . . 85

4.6 .1 Structure . . . . . . . . . . . . 86

4.6.2 Density of states . . . . . . . . . . . . . 89

4.7 UV-visible spectroscopy . . . . . . . . . . . . . . . 90

4.7.1 Powder diffuse reflectance . . . . . . . . . . . . . . 91

4.7.2 Spectroscopy of thin films . . . . . . . . . . . . 92

5 Transition Metal Hybrids $\quad 95$

5.1 Synthesis . . . . . . . . . . . . . . . . . 96 
5.1.1 Comparison of methods . . . . . . . . . . . . 99

5.2 Results . . . . . . . . . . . . . . . . . . 103

5.2 .1 X-ray diffraction . . . . . . . . . . . . . 103

5.2 .2 Electron microscopy . . . . . . . . . . . . . . . 108

5.2.3 Elemental microanalysis . . . . . . . . . . . . . 113

5.2.4 IR and Raman spectroscopy . . . . . . . . . . . . . . 114

5.2.5 XANES and EXAFS . . . . . . . . . . . 117

5.2.6 UV-visible spectroscopy . . . . . . . . . . . . . 123

5.3 Summary . . . . . . . . . . . . . . . . . . . . 128

6 Magnetic Properties 131

6.1 Introduction to magnetic behaviour . . . . . . . . . . . . . 131

6.2 Low-dimensional models . . . . . . . . . . . . . . . . . . 134

6.3 Tungsten oxide hybrids . . . . . . . . . . . . . . . . 136

6.4 Transition metal tungstate hybrids . . . . . . . . . . 137

6.4.1 Manganese tungstate hybrids . . . . . . . . . . 138

6.4.2 Iron tungstate hybrids . . . . . . . . . . . . . . . 142

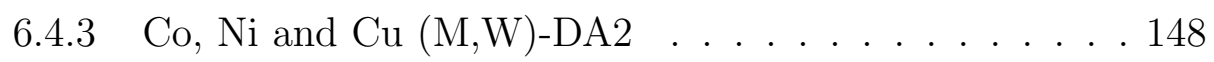

6.4.4 Copper tungstate hybrid . . . . . . . . . . . 150

6.4.5 Nickel tungstate hybrid . . . . . . . . . . . . . 153

6.5 Summary . . . . . . . . . . . . . . . . . 155

7 Doping $\quad 159$

7.1 Background . . . . . . . . . . . . . . . . . . 159

7.2 Previous studies . . . . . . . . . . . . . . . . . . . . 162

7.3 Electrochemical Doping. . . . . . . . . . . . . . 163

7.3.1 $\mathrm{WO}_{3} \ldots \ldots \ldots \ldots \ldots$

7.3.2 $\mathrm{H}_{2} \mathrm{WO}_{4} \ldots \ldots \ldots \ldots \ldots . \ldots \ldots$

7.3.3 W-DA2 and W-DA6 hybrids . . . . . . . . . 168

7.4 Summary . . . . . . . . . . . . . . . . . 171

8 Outlook and Conclusion $\quad 173$

8.1 Tungsten oxide hybrids . . . . . . . . . . . . . . . . . 173

8.2 Transition metal tungstates . . . . . . . . . . . 176 
8.3 Magnetism . . . . . . . . . . . . . . . . . . 177

8.4 Doping . . . . . . . . . . . . . . . . . . . 179

8.5 Soft-mode behaviour . . . . . . . . . . . . . . . . . . . 179

8.6 Conclusion . . . . . . . . . . . . . . . . . . . . 180

A Literature Examples of Hybrid Systems 183

B VASP Input Files 189 


\section{List of Tables}

1 Abbreviations of organic molecules ... . . . . . . . viii

2 Abbreviations of experimental techniques . . . . . . . . ix

2.1 Solvents used to dissolve organic reagents . . . . . . . . . . . 27

3.1 Synthesis methods for various intercalates . . . . . . . . . 37

3.2 Microanalytical results for W-DAn hybrids . . . . . . . . . . 46

3.3 Thermogravimetric analysis . . . . . . . . . . . . . . . . 48

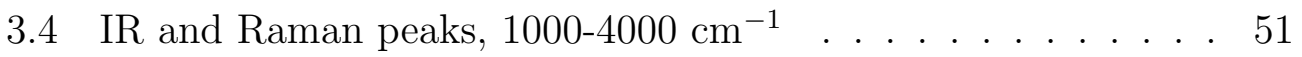

3.5 IR and Raman peaks, $0-1000 \mathrm{~cm}^{-1} \ldots . . . . . . . .552$

3.6 W-MAn IR peaks . . . . . . . . . . . . . . . 57

4.1 Valence states . . . . . . . . . . . . . . . . . . 67

4.2 Cell parameters for cubic and hexagonal tungsten bronzes . . 71

4.3 Calculated cell volume for tungsten bronzes . . . . . . . . . 72

4.4 Energies of formation of tungsten bronzes . . . . . . . . . 73

4.5 Compositional ranges of tungsten oxide species . . . . . . . . . 82

4.6 Energies of formation of $\mathrm{WO}_{3-x} \ldots \ldots \ldots$. . . . . . . 83

4.7 Calculated cell parameters of W-DA2 . . . . . . . . . . 88

4.8 Energies of formation of W-DAn . . . . . . . . . . . . 89

4.9 UV-visible spectra of powder samples . . . . . . . . . . . . . 92

5.1 Synthesis methods for transition metal tungstate hybrids . . . 100

5.2 Elemental analysis results for transition metal hybrids . . . . . 114

5.3 EXAFS results for manganese tungstate hybrids . . . . . . . . 118

6.1 Weiss temperatures for transition metal tungstate hybrids . . 138 
6.2 Curie-Weiss parameters for (M,W)-DA2 hybrids . . . . . . . . 149

7.1 Electrochemically intercalated $\mathrm{H}_{2} \mathrm{WO}_{4} \ldots \ldots$. . . . . 167

A.1 Abbreviations of techniques used in this section . . . . . . . 183

A.2 Abbreviations of organic molecules used in this section. . . . . 184

A.3 Examples of organic-inorganic hybrids . . . . . . . . . . . 186

A.4 Examples of $\mathrm{MX}_{4}$-based hybrids . . . . . . . . . . . . . . 187 


\section{List of Figures}

1.1 Structure of cubic tungsten bronze . . . . . . . . . . . 16

1.2 Structure of tetragonal tungsten trioxide . . . . . . . . . . 19

1.3 Structure of hexagonal tungsten trioxide . . . . . . . . . . . 20

1.4 Structure of pyrochlore tungsten trioxide . . . . . . . . . 20

1.5 Structure of tungstic acid, $\mathrm{H}_{2} \mathrm{WO}_{4} \ldots \ldots \ldots$

3.1 XRD comparison of synthesis methods . . . . . . . . . 37

3.2 SEM comparison of synthesis methods . . . . . . . . . 38

3.3 IR comparison of synthesis methods . . . . . . . . . . . . . . 39

3.4 XRD patterns of the W-DAn series . . . . . . . . . . . . 42

3.5 Diagram of W-DAn structure . . . . . . . . . . . . . . 43

3.6 Electron diffraction images of W-DAn . . . . . . . . . . . . 45

3.7 Thermogravimetry of W-DAn . . . . . . . . . . 47

3.8 Infrared spectra of tungsten oxide-related materials . . . . . . 50

3.9 Raman spectra of tungsten oxide-related materials . . . . . . . 50

$3.10 \mathrm{pK}_{a}$ versus number of carbon atoms in DAn . . . . . . . . 55

3.11 Infrared spectra of W-MAn hybrids . . . . . . . . . . . 58

4.1 Cell parameter of cubic tungsten bronzes . . . . . . . . . . 70

4.2 Charge density maps of tungsten bronzes . . . . . . . . . . 73

4.3 Energies of formation of tungsten bronzes . . . . . . . . . . 74

4.4 Density of states for cubic and hexagonal $\mathrm{WO}_{3} \ldots \ldots$. . . . 76

4.5 Density of states for cubic tungsten bronzes . . . . . . . . 78

4.6 Band structure diagrams of cubic $\mathrm{WO}_{3}$ and $\mathrm{NaWO}_{3} \ldots \ldots .79$

4.7 Cell parameter of cubic $\mathrm{Na}_{x} \mathrm{WO}_{3}$. . . . . . . . . . . 80 
4.8 Band structure of $\mathrm{Na}_{x} \mathrm{WO}_{3}$ near the Fermi level . . . . . . . . 81

4.9 Cell parameter for $\mathrm{WO}_{3-x} \ldots \ldots \ldots$. . . . . . . . . . . 82

4.10 Density of states for $\mathrm{WO}_{3-x} \ldots \ldots \ldots$. . . . . . . . . 84

4.11 Two bonding schemes of hybrids . . . . . . . . . . . . . 86

4.12 Calculated structure of W-MA1 . . . . . . . . . . . . . 87

4.13 Calculated structure of W-DA2 . . . . . . . . . . . 88

4.14 Density of states for W-DAn . . . . . . . . . . . . . 90

$4.15 \mathrm{UV}$-visible spectra of tungsten oxide-based materials . . . . . 91

4.16 Absorption coefficient analysis of thin films . . . . . . . . . . . 93

5.1 XRD comparison of (Mn,W)-DA6 via two synthesis methods . 96

5.2 XRD patterns of transition metal tungstate hydrates . . . . . 98

5.3 SEM micrographs of transition metal tungstate hydrates . . . 98

5.4 Infrared spectra of transition metal tungstate hydrates . . . . 99

5.5 SEM comparison of transition metal hybrids . . . . . . . . . 101

5.6 XRD comparison of synthesis methods . . . . . . . . . . . . . 102

5.7 XRD patterns of various hybrids with long organic intercalates 104

5.8 XRD patterns of $(\mathrm{Mn}, \mathrm{W})$-DAn hybrids . . . . . . . . . . . 105

5.9 Diagram of $(\mathrm{Mn}, \mathrm{W})-\mathrm{DAn}$ structure . . . . . . . . . . 106

5.10 XRD pattern of $(\mathrm{Mn}, \mathrm{W})$-DAphen . . . . . . . . . . . . 108

5.11 XRD patterns of transition metal hybrids, (M,W)-DA2 . . . . 109

5.12 XRD patterns of transition metal hybrids, (M,W)-DA6 . . . . 109

5.13 SEM micrographs of transition metal tungstate hybrids . . . . 110

5.14 High-resolution TEM image of (Mn,W)-DA6 . . . . . . . . . . 112

5.15 Electron diffraction image of $(\mathrm{Mn}, \mathrm{W})-\mathrm{DA} 6 \ldots . . . .113$

5.16 Infrared spectra of manganese tungstate-based materials . . . 115

5.17 Raman spectra of manganese tungstate-based materials . . . . 115

5.18 XANES spectra of (Mn,W)-DAn at the W L1-edge . . . . . . 117

5.19 XANES spectra of $(\mathrm{Mn}, \mathrm{W})$-DAn at the Mn K-edge . . . . . . 118

5.20 EXAFS spectra for $(\mathrm{Mn}, \mathrm{W})$-DAn from the W L1-edge . . . . 119

5.21 EXAFS spectra for $(\mathrm{Mn}, \mathrm{W})$-DAn from the Mn K-edge . . . . 119

5.22 Proposed structure for $(\mathrm{Mn}, \mathrm{W})-\mathrm{DAn} \ldots \ldots$. . . . . . . . . 121

$5.23 \mathrm{UV}$-visible spectra of Mn and Fe tungstate hybrids . . . . . . 124 
5.24 UV-visible spectra of Co and Ni tungstate hybrids . . . . . . . 125

$5.25 \mathrm{UV}$-visible spectra of $\mathrm{Cu}$ tungstate hybrids . . . . . . . . . 125

6.1 DC magnetisation of W-DA6 . . . . . . . . . . 137

6.2 Values of $P^{e f f}$ for transition metal tungstate hybrids . . . . . 139

6.3 DC susceptibility of $(\mathrm{Mn}, \mathrm{W})$-DAn series . . . . . . . . . . 139

6.4 Spin-ladder diagram . . . . . . . . . . . . . . . . . . 140

6.5 DC susceptibility of (Mn,W)-DA2 with fitting curves . . . . . 141

$6.6 \mathrm{M} / \mathrm{H}$ curves for $(\mathrm{Fe}, \mathrm{W})-\mathrm{DAn}$ series . . . . . . . . . . 143

6.7 AC susceptibility for $(\mathrm{Fe}, \mathrm{W})-\mathrm{DAn}$ series . . . . . . . . . . . 144

6.8 Hysteresis loops of $(\mathrm{Fe}, \mathrm{W})-\mathrm{DA} 2$ and $(\mathrm{Fe}, \mathrm{W})-\mathrm{DA} 6 \ldots . . .146$

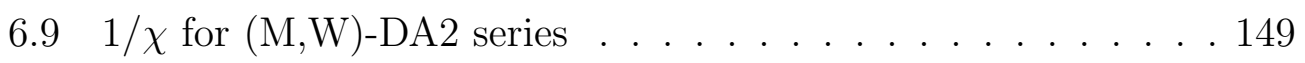

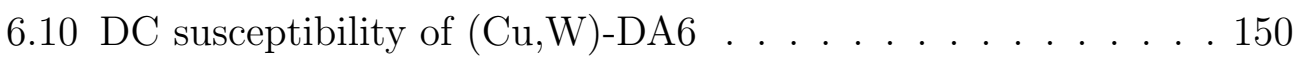

6.11 DC $M / H$ curves for $(\mathrm{Ni}, \mathrm{W})-\mathrm{DA} 6 \ldots \ldots$

6.12 Hysteresis loops of $(\mathrm{Ni}, \mathrm{W})-\mathrm{DA} 6 \ldots \ldots$. . . . . . . . . . 154

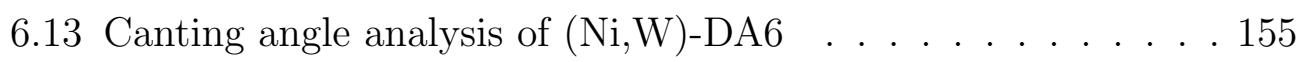

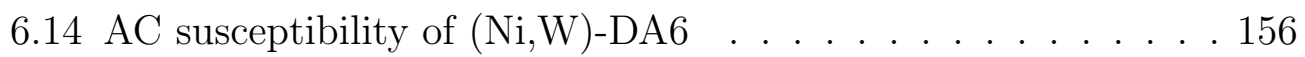

7.1 Cyclic voltammetry curves of $\mathrm{WO}_{3} \ldots \ldots$. . . . . . . 164

7.2 XRD patterns of electrochemically intercalated $\mathrm{Na}_{x} \mathrm{WO}_{3}$. . 165

7.3 Cyclic voltammetry curves of $\mathrm{H}_{2} \mathrm{WO}_{4} \ldots \ldots$. . . . . . 166

7.4 XRD patterns of electrochemically intercalated $\mathrm{H}_{2} \mathrm{WO}_{4}$. . . 167

7.5 Cyclic voltammetry curves of W-DAn with $\mathrm{NaCl}$. . . . . . . 168

7.6 Cyclic voltammetry curves of W-DAn with $\mathrm{H}_{2} \mathrm{SO}_{4} \ldots \ldots$ 


\section{Chapter 1}

\section{Introduction}

This thesis describes the synthesis and characterisation of novel organicinorganic hybrid materials based on atomic layers of tungsten oxide separated by organic amine spacer molecules. The inorganic layers are, in effect, isolated by the insulating organic 'spacer' molecules, and thus are expected to behave as a two-dimensional system with little or no electronic coupling in the direction perpendicular to the layers (although in principle conducting organic molecules could be also utilised). This makes for an interesting physical system, particularly if one is able to dope the inorganic layers and induce conductivity within the two-dimensional planes.

This chapter will explore some motivations for studying the system in question, beginning with a survey of various phenomena observed in lowdimensional and strongly correlated electronic systems. Section Two will provide some background on historical and recent reports in the very broad field of organic-inorganic hybrid materials. Section Three will discuss the physical and chemical properties of tungsten oxide systems, which form the basis for the layered organic-inorganic materials discussed throughout this thesis. 


\subsection{Strongly Correlated Electronic Systems}

\subsubsection{Basic Electronic Theory in Solids}

A systematic survey of electronic behaviour in solids given in solid state textbooks (e.g. [9]) usually begins by considering the free electron model with the Drude and then the Sommerfeld theories.

The Drude theory was proposed in 1900 for the electrical and thermal conduction behaviour of metals, considering the metal as a 'gas' of electrons and applying the known kinetic theory of gases to this. Positive charges were assumed to be immobile and consisted of what is now known as the nucleus plus the core electrons. The valence (or conduction) electrons were free to move throughout the solid and had a compensating negative charge so the charge on the metal is neutral overall. Electron-electron and electron-nucleus interactions are neglected, except for collisions, which last for a negligible amount of time and occur at an average interval, $\tau$, which is independent of the position and velocity of the electrons. This leads to the fundamental relations for the frequency-dependent conductivity

$$
\sigma(\omega)=\sigma_{0}(1+j \omega \tau)^{-1}
$$

where the DC conductivity $\sigma_{0}$ is given by

$$
\sigma_{0}=n e^{2} \tau / m
$$

$n$ is the volume density of electrons and $m$ is their mass. The form of Equation 1.1 results in a so-called 'Drude peak' occurring in the conductivity at low frequency. In optical spectroscopy this is interpreted as a signature of free-carrier density, namely metallic character.

Because the Drude theory is based on the classical kinetics of gases, it uses the Maxwell-Boltzmann distribution to describe the velocity of the electrons at a given temperature. However, quantum theory demonstrates that electrons are subject to the Pauli exclusion principle. In 1928 Sommerfeld proposed that the Maxwell-Boltzmann distribution be replaced with the FermiDirac distribution to account for the quantum nature underlying the energy of electrons in a system. 
In most real systems, electrons do not behave as a non-interacting gas but are influenced by each other to varying degrees and by the periodic ionic lattice of the solid itself. This consideration led to the development of Bloch's theorem in 1928 [28].

Bloch's theorem states that the eigenstates $\psi$ of the Hamiltonian $H$ for a single electron in a periodic potential can be chosen to have the form of a plane wave multiplied by a function corresponding to the periodicity of the Bravais lattice. Thus, Schrödinger's equation

$$
H \psi=-\left(\frac{\hbar^{2}}{2 m} \nabla^{2}+U(\mathbf{r})\right) \psi=\varepsilon \psi
$$

is satisfied by

$$
\psi_{n \mathbf{k}}(\mathbf{r})=e^{i \mathbf{k} \cdot \mathbf{r}} u_{n \mathbf{k}}(\mathbf{r})
$$

where both

$$
u_{n \mathbf{k}}(\mathbf{r}+\mathbf{R})=u_{n \mathbf{k}}(\mathbf{r})
$$

and

$$
U(\mathbf{r})=U(\mathbf{r}+\mathbf{R})
$$

in Equation 1.2 hold for all $\mathbf{R}$ in the Bravais lattice.

Bloch's theorem also led to the concept of energy bands. Due to the Pauli exclusion principle, electrons will occupy states $\psi_{n \mathbf{k}}$ of energies $\varepsilon_{n \mathbf{k}}$ for each $n$, up to the number of electrons. When all electrons occupy their lowest possible states, the highest occupied energy is then denoted the Fermi level or Fermi energy, $\varepsilon_{F}$.

If the Fermi level lies between a completely filled valence band and a completely empty conduction band, then the resulting material is rendered semiconducting, with a band gap defined as the difference in energy between the top of the highest filled band and the bottom of the lowest empty band. ${ }^{1}$

\footnotetext{
${ }^{1}$ These bands have several different names; in physics - and therefore throughout this thesis - the highest filled band is called the valence band, and the lowest empty band is called the conduction band. In chemistry, the former is called the HOMO (highest occupied molecular orbital) and the latter is called the LUMO (lowest unoccupied molecular orbital).
} 
Electrons can be stimulated across the band gap by increasing their energy via photons (X-rays, UV etc.), phonons (temperature), or externally applied electric or magnetic fields.

If the Fermi level lies within a partially-filled band (or bands), the system has conducting (or metallic) properties. In these band(s) a constant-energy surface can be found in $k$-space that separates occupied and unoccupied levels. This surface is called the Fermi surface, and the energy is the Fermi energy.

In many 'normal' metals the weak repulsive electron-electron Coulombic interactions are 'screened' by the presence of the positive ions in the crystal. This results in the electron-electron repulsion being damped exponentially so that it is negligible over distances of the order of the ionic spacing. Mathematical formalism describes such systems in terms of quasiparticles. These are not physical particles like electrons, but there is a one-to-one correspondence between the number of quasiparticles and electrons, and over short distances they interact like very weak electrons.

Up until this point, the assumption has been made that the electronelectron interaction is small and can be treated perturbationally. For ideal systems, and some real ones, this is sufficient to describe their electronic behaviour. However for the vast majority of real systems the electron-electron interaction is not just a first-order correction, and this increases the complexity of the model required. Such systems where the electron-electron interaction is significant are called strongly correlated electronic systems.

\subsubsection{Examples}

To fully describe strongly correlated electronic systems would require the many-body problem to be solved exactly. The lack of any such exact solution makes understanding these systems one of the most challenging aspects of solid state physics.

Strong electronic interactions lead to a rich variety of possible phases, each of which could be exemplified in the present hybrid materials, under 
suitable circumstances. Some examples of such systems and their behaviour are as follows:

\section{Magnetic materials}

Magnetism arises, in general, from the presence of unpaired electrons associated with a single nucleus, resulting in a net spin excess. When considering the spins of electrons, the Pauli exclusion principle is complemented by Hund's Rules. These allow us to determine the lowest energy magnetic state with appropriate quantum numbers $S, L$, and $J$, describing the spin, orbital and total angular momentum of the atom or ion, respectively. Once again, only the valence electrons contribute to the observed behaviour (magnetism), because the lower-energy core electrons are paired in alternate spins $(\uparrow \downarrow)$ because of the Pauli exclusion principle. There can also be magnetic interaction between the unpaired electrons and the nucleus, resulting in hyperfine magnetic field phenomena.

Within the broad scope of 'magnetic materials' there are many different behaviours observed. The two most common types of magnetically ordered systems are ferromagnetic, where all spins point in the same direction; and antiferromagnetic, where (as the name suggests) adjacent spins alternate direction in one or more dimensions. Magnetic correlations are discussed in more detail in Chapter 6 .

\section{Quantum Hall effect}

The Nobel Prize in Physics in 1985 was awarded to Klaus von Klitzing for the discovery of the quantum Hall effect [269]. In the classical Hall effect, when a magnetic field is applied perpendicular to a current flowing through a metal, a transverse voltage is observed in the third orthogonal direction that is proportional to the current and the applied field. At low temperatures $(O(m K))$ the Hall resistance is observed to increase in discrete quanta as the field is increased (hence, the quantum Hall effect).

The phenomenon can be explained by the formation of Landau levels which alter the density of states. In an applied magnetic field the electrons' 
trajectories will be a series of concentric circles about the axis of the field, and will be quantised according to the cyclotron frequency $\omega_{c}=e B / m^{*}$. The density of states reverts to a series of discrete levels which are smeared out with temperature, and their positions are dependent on the applied field. As the field increases, a Landau level may pass through the Fermi energy and only at that point is conduction possible.

\section{Heavy Fermion compounds}

The electronic heat capacity can be calculated from the free-electron Fermi gas model. In most systems the electronic heat capacity is much lower than the atomic heat capacity, and the electronic contribution can normally only be observed at low temperatures. At low temperatures the heat capacity of the total system is written as $C=\gamma T+A T^{3}$, namely the sum of the linear electronic contribution and the phonon contribution. $\gamma$ is expected to have a value of $\frac{1}{2} \pi^{2} N k_{B} / T_{F}$ (where $T_{F}$ is the 'Fermi temperature', $k_{B} T_{F} \equiv \varepsilon_{F}$ ) and while most systems are within the correct order of magnitude, they do not agree closely. It is common practice to assign to the electrons an effective mass, $m^{*}$, relative to the electron mass, given by the ratio of the observed and expected $\gamma$ values.

Some metallic compounds (many including cerium) have been observed to have effective electronic masses several orders of magnitude greater than the electronic mass $[214,252,253]$. This effect is thought to be due to weak overlapping of the $f$-electron wavefunctions on adjacent ions within the compound, leading to strong electron-electron interactions. The free carrier density can be changed by the application of pressure and the temperaturepressure phase diagram is generally rich, revealing various magneticallyordered states as well as superconductivity [191].

\section{Bose-Einstein condensates and Mott insulators}

Atoms or particles cooled to ultra-low temperatures can exhibit one of two ground states: Bose-Einstein condensates or Mott insulators, depending on the strength of the atomic interactions. In a Bose-Einstein condensate the 
very weak repulsive interactions between the atoms allow all the wavefunctions of the particles to overlap coherently over the entire group of particles. Each one then behaves as a wave spread over the entire lattice [4, 221].

Mott insulators are almost the complete opposite, in that the atoms behave as particles confined to their appropriate lattice sites. The repulsive interactions between them are strong enough to prevent the movement of electrons through the lattice.

For example, cuprate high-temperature superconductors at low or zero doping are naïvely expected to have a half-filled valence band and therefore be metallic. (This is the $t_{2 g}$ band, the highest band arising from crystalfield-splitting.) But the strong Coulomb repulsion associated with an electron hopping to an adjacent site leads to a splitting of the band into an empty upper Mott-Hubbard band and a filled lower Mott-Hubbard band. The system is then an antiferromagnetic insulator [5].

\section{Kondo effect}

The Kondo effect refers to a phenomenon seen in the resistance of magnetic alloys, which instead of decreasing monotonically with decreasing temperature, has been observed to decrease to a broad minimum at low temperatures and then rise again, dependent on the concentration of magnetic species.

This was explained by J. Kondo in 1963 [141] in relation to the scattering of conduction electrons from the magnetic atoms. There is an exchange between the two which results in a scattering event where the spin of the electron is flipped. This behaviour dominates at the lowest temperatures as the phonon contribution normally responsible for resistance becomes sufficiently small. The temperature (or energy) scale, $T_{K}$, is set by the Fermi temperature $T_{F}$, the exchange coupling constant $J$ and the population of the density of states at the Fermi level [265].

\section{Colossal magneto-resistance}

Colossal magnetoresistance (CMR) is a property of many perovskite manganites, $\mathrm{A}_{x} \mathrm{~B}_{1-x} \mathrm{MnO}_{3}$, where, at a certain temperature, the resistivity can 
be observed to increase by many orders of magnitude when a relatively small magnetic field is applied [76, 154, 227, 238]. At this temperature a paramagnetic to ferromagnetic transition is observed, along with an insulator to metal transition. The composition, $x$, is chosen to lie close to a magnetic phase transition boundary which is traversed by the application of a magnetic field. Such materials have applications as magnetic recording devices.

An explanation of this effect is still being sought. There is evidence to suggest it is due in part to interactions between $\mathrm{Mn}^{3+}$ and $\mathrm{Mn}^{4+}$ ions, the concentrations of which are determined by the doping level $x$. However, this mechanism ('double exchange') alone is not enough to predict the high resistivities observed. There are also thought to be electron-phonon interactions due to Jahn-Teller distortions of the manganese ions [194].

\section{Luttinger liquids}

Luttinger liquids are a theoretical low-dimensional counterpart to Fermi liquids, where the particles behave in a collective manner that is different from that of the original particles [5]. In the electronic case, electrons (which are fermions) in a Luttinger liquid do not behave as fermions but rather as bosons. Two systems thought to resemble Luttinger liquids are conducting polymers and carbon nanotubes. Both of these display additional interesting low-dimensional properties due to their 1-dimensional structure.

Carbon nanotubes in particular have a wide range of electronic properties which vary from semiconducting to metallic depending on their chirality [11]. They have been observed to display ballistic electron transport, that is, the conductance is free of scattering and hence does not depend on the length of the tube [280]. This is due to the interaction of electrons in a delocalised, coherent fashion along the nanotube.

\section{High-temperature superconductivity}

High-temperature superconductors (HTS) are perhaps the epitome of strongly correlated electronic systems, and are certainly the most extensively studied [259]. Superconductivity arises through the formation of Cooper pairs, 
where electrons with opposing spins and momenta pair up to form a bosonlike quasiparticle. Unlike fermions, bosons are not restricted as to how many may occupy the ground state and therefore all the Cooper pairs condense to the lowest-lying energy state at the Fermi level, establishing phase coherence throughout the entire sample (which may be kilometres long!). This coherence allows the electrons (as Cooper pairs) to move throughout the lattice without scattering and hence without resistance.

Most high-temperature superconductors are based on structures with one or more corner-shared square planar copper oxygen $\mathrm{CuO}_{2}$ planes separated by (essentially) insulating layers. It is also possible in systems such as $\mathrm{Ru}_{2} \mathrm{Sr}_{2} \mathrm{RECu}_{2} \mathrm{O}_{8}(\mathrm{RE}=$ rare earth $)$ to have magnetism present in the $\mathrm{RuO}_{2}$ layers that competes or even co-exists with the superconductivity in the $\mathrm{CuO}_{2}$ layers [25, 223].

In each of these cases the phenomena observed are due to the significant interactions between electrons and electrons, electrons and ions, or electrons and phonons. They cannot be explained by any one simple model using standard perturbation approaches.

Another point to note is that, particularly in systems such as carbon nanotubes and HTS cuprates, the low-dimensionality of the system plays an important part in its observed properties. Carbon nanotubes, metal nanowires and conducting polymers are examples of quasi-one-dimensional systems. HTS cuprates, thin film multilayers, self-assembled monolayers (SAMs) and, as we shall see, certain organic-inorganic layered hybrid materials, are all examples of quasi-two-dimensional systems - that is, properties such as electron transport and magnetism operate with little or no coupling between layers, at least in certain energy (e.g. temperature) regimes. Each layer is nearly independent and isolated from any adjacent layers. These behaviours can often be easily explained by a 1- or 2-dimensional model with simple temperaturedependent coupling. An alternative way of viewing such systems is to assign an anisotropic effective mass tensor. For in-plane transport $m^{*}$ may be close to the electronic mass, $m_{e}$, while for the out-of-plane transport, $m^{*}$ may be much larger. 
One method of designing a system to have low dimensionality is to separate an inorganic material in a low-dimensional form by insulating organic molecules. These molecules may have functionality themselves or may simply be space-filling. We will now discuss some aspects of the broad range of compounds classed as 'organic-inorganic hybrid materials'.

\subsection{Organic-Inorganic Hybrid Materials}

The concept of organic-inorganic hybrid materials is based upon combining the aspects of the two fundamental ingredients: the electrical, magnetic, structural and thermal stability properties of the inorganic part with the flexibility, functionality and templating ability of the organic part. An analogy can be drawn in that the properties of various alloys are often much better than those of the individual metals of which they are composed. Different hybrid materials have been the subject of research in semiconducting electronics, gas/molecule sensors, drug/biomolecule capture and delivery, superconductivity, and many other areas.

In literature, the title 'organic-inorganic hybrid material' is used very broadly and has been applied to systems ranging from metal-organic complexes to macrostructural inorganic particles in an organic matrix. Between these two extremes, organic-inorganic components can be found which are comprised of molecular units in 3-dimensional networks, 2-dimensional layered structures, 1-dimensional chains or even 0-dimensional clusters. Of these, one can find a variety of systems from examples where the organic component is used merely as a templating agent for the inorganic layer, to cases where several different organic species are used in a structural sense via co-ordination to the inorganic species. The organic molecule can be chosen to have a broad range of functionality, provided it can be incorporated within the inorganic structure. The lower the dimensionality of the inorganic framework, the greater the variety of organic molecules that can be included. The bonding nature between the organic and inorganic species also varies in strength from very weak van der Waals bonding to ionic and co-ordination (or 'direct') bonds. The bond strength impacts upon the structure and elec- 
tronic properties of the system.

Various classes of organic molecules can be chosen to provide the resulting hybrid with specific functionality - for example, polymerisable species (for structural and/or electronic cross-linking), conjugated molecules (for extra conduction and enhanced interlayer coupling), dyes, chromophores, and luminescent species (for non-linear optical responses), and others. Organic molecules can range in complexity from simple straight-chain (saturated) alkanes and aromatic molecules through to organic dyes, polymers, and biomolecules. In some cases the organic molecule acts merely as a templating agent and is later removed to form a porous material; such systems will not be discussed here. Also not discussed here are "macro-scale" systems consisting of inorganic nanoparticles in an organic matrix, and the many different Si-O-based systems, which, for the most part, are structurally and chemically similar to C-based systems.

There is a variety of synthesis methods utilised to make the various hybrid materials, including slow crystallisation from solution, evaporation of solvent, hydro- or solvo-thermal methods, chimie douce ("soft chemistry"), gas-phase reactions, sol-gel precursors, electrochemical crystal growth, and solid state reactions. A selection of hybrid systems with their dimensionality, bonding nature and synthesis methods is shown in Appendix A. These have been separated into several major categories, as follows:

\section{Phosphate-based organic/inorganic hybrid materials}

These are normally synthesised using hydro- or solvo-thermal methods and typically form as networks (1-, 2- or 3-D) of metal atoms linked via the oxygen atoms of the $\mathrm{PO}_{4}{ }^{3-}$ tetrahedra linkages $[137,249]$. Aminoalkanes are the most common organic intercalate [81]. The size of the organic molecules and the synthesis conditions ( $\mathrm{pH}$, temperature, pressure, concentrations) have a strong influence on the structure of the inorganic layer. 


\section{Expanded perovskite-based organic/inorganic hybrid materials}

Many different layered perovskites have been used as the inorganic layers in organic-inorganic hybrids formed by chimie douce methods. These include the perovskite $\mathrm{Ca}_{2} \mathrm{Nb}_{3} \mathrm{O}_{10}$ and its derivatives [122], the Aurivillius phases $\mathrm{Bi}_{2} \mathrm{~W}_{n} \mathrm{O}_{3 n+3}$ [152], the $\mathrm{MnO}_{2}$ 'slab' structures birnessite and buserite [88, 175], the layered double hydroxide (LDH) system, based on alumino-silicate clays [131], and even the HTS $\mathrm{Bi}_{2} \mathrm{Sr}_{2} \mathrm{Ca}_{n-1} \mathrm{Cu}_{n} \mathrm{O}_{2 n+4}$ superconductor series $[50,52,53,80,139]$. The organic molecules act primarily to separate the inorganic layers, and they may be present as neutral species or positively charged to maintain charge balance.

\section{Hybrids based on cluster ions}

"Cluster ions" is a broad term used here to refer to heteropoly anions, including Keggin ions and Wells-Dawson structures, which consist of clusters of bonded $\mathrm{MO}_{6}$ octahedra $(\mathrm{M}=\mathrm{Mo}$ or $\mathrm{W})$. Such structures normally form at low pH synthesis conditions $[134,254]$ and in hybrid materials are connected via the organic molecules. These are essentially zero-dimensional systems as the cluster ions for the most part do not interact with each other. The hybrids may be produced via a range of techniques, including hydrothermal synthesis [230], precipitation from solution [244], sol-gel method [295], or successive layers built up by dip-coating [273].

\section{Other miscellaneous systems}

There are of course many other possible combinations for forming organicinorganic hybrid materials, however in literature these appear to be for the most part individual structures that are not part of any series, in contrast to the other 'classes' listed here. One system worthy of mention however is the vanadate hybrid structures, which are able to form a variety of structures, most of them quite complicated. Ref. [98] gives an extensive review of these vanadate hybrids. 


\section{Metal halide layer-based hybrids}

There are a wide variety of these materials, which are comprised of $\mathrm{MX}_{6}$ octahedra which are corner-shared to form simple regular 2-dimensional sheets, $\mathrm{MX}_{4}{ }^{2-}\left(\mathrm{M}=\mathrm{M}^{2+}\right.$ metal ion, $\left.\mathrm{X}=\mathrm{Cl}, \mathrm{Br}, \mathrm{I}\right)$. The layers are then separated by amine-terminated organic molecules. Ref [196] is an excellent review of these materials, displaying the wide variety of inorganic species and organic amine molecules that together can form this relatively simple structure. These systems have been studied for over 70 years [232] and have been shown to display remarkable photoluminescence [79, 202] and have been applied as materials in thin film transistors [130]. Their magnetic properties have also been extensively studied and represent a near-perfect 2-dimensional magnetic system $[71,72,206,264]$.

Layered metal halide-based hybrid materials have been synthesised with the halides $\mathrm{Cl}, \mathrm{Br}$ and I, and the metal ions thus far incorporated include the transition metals $\mathrm{Cr}, \mathrm{Mn}, \mathrm{Fe}, \mathrm{Ni}, \mathrm{Cu}, \mathrm{Pd}, \mathrm{Cd}$ [196]; Group 14 metals Ge, $\mathrm{Sn}, \mathrm{Pb}$ [195, 196]; and rare earth Eu [202]. Copper halide compounds in particular demonstrate a wide variety of possible structures due to Jahn-Teller distortions [283]. It is also possible to vary the thickness of the inorganic layer, and above approximately three inorganic octahedral layers the materials change from semiconducting to metallic [200].

The simplest compounds incorporate straight-chain mono- or di-aminoalkanes as the organic component, whose primary function is to vary the inorganic interlayer spacing. Electron-rich conjugated (aromatic and aryl) organics can alter the electrical properties of the hybrids through doping effects. Organic dyes may add chromogenic properties, and polymerisable molecules provide additional cohesion, and also the possibility of introducing conducting polymers [46, 196].

The nature of the bonding is influenced by the length of the organic molecule (affecting charge separation), its saturation (relating to delocalised electrons) and the terminal group used to bind to the inorganic layer (ammonium ion, pyridinium ion etc.). The bonding nature also impacts on the structure of the hybrid as most organics will then be oriented at a partic- 
ular angle from the normal, which is the most energetically favoured. The metal halide hybrids are normally synthesised by dissolving stoichiometric amounts of the metal halide $\mathrm{MX}_{2}$ salt and the organic (or organic salt) separately in the appropriate concentrated acid HX, with heating and under an inert atmosphere. When both are dissolved the solutions are combined, and a crystalline precipitate is formed. Thin films for device testing can be made by dissolving the product in an appropriate solvent and spin-coating [130] or dip-coating [171]; by melt-processing [199], or by single- or dual-source thermal ablation $[78,205]$. The desired functionality is achieved by choosing an appropriate organic molecule.

One major limitation of the metal halide-organic amine system is that the compounds are easily oxidised in air and therefore must be synthesised, stored and handled under an inert atmosphere. If one were able to form an identical structure with an appropriate metal oxide, this issue could be overcome. Such an oxide structure in its simplest form requires a hexavalent metal ion. This can be seen by ionic counting. The square-planar, cornershared assembly of $\mathrm{WO}_{6}$ octahedra has the formula $\mathrm{MO}_{4}$ to which two R$\mathrm{NH}_{3}{ }^{+}$groups are added. Charge balance for $\mathrm{MO}_{4}\left(\mathrm{RNH}_{3}{ }^{+}\right)_{2}$ requires $\mathrm{M}^{6+}$. Hence, this nominally limits such structures to molybdenum and tungsten oxides.

Molybdenum and tungsten oxides can form layered hybrid materials, and the oxide systems themselves display interesting properties. This is discussed in detail in the following section.

\subsection{Tungsten Oxide Systems}

Tungsten trioxide, $\mathrm{WO}_{3}$, has long been studied for its interesting structural, electronic, and electrochromic properties. The basic structure of tungsten trioxide consists of a three-dimensional network of $\mathrm{WO}_{6}$ octahedra, shared at all their corners, otherwise known as the $\mathrm{ReO}_{3}$ structure. However, unlike $\mathrm{ReO}_{3}$ which exhibits a high degree of symmetry and hence a simple cubic cell, the $\mathrm{WO}_{6}$ octahedra are slightly distorted in terms of $\mathrm{W}-\mathrm{O}$ bond lengths 
and $\mathrm{W}-\mathrm{O}-\mathrm{W}$ bond angles, due to antiferroelectric displacement of the $\mathrm{W}$ atoms and subsequent rotation of the $\mathrm{WO}_{6}$ octahedra [35, 82]. At room temperature tungsten trioxide adopts a monoclinic cell, but exhibits phase changes as the temperature is increased or decreased [163, 233].

The structure of $\mathrm{WO}_{3} / \mathrm{ReO}_{3}$ can also be described as a perovskite structure, $\mathrm{ABO}_{3}$. The basic definition of the perovskite structure is a cubic cell, with $\mathrm{A}$ atoms occupying the corner sites, $\mathrm{O}$ atoms in a face-centred cubic arrangement, and a $\mathrm{B}$ atom (in this case, tungsten) occupying the body centre. When the A site is fully or partially occupied by a metal ion, as in Figure 1.1, this is called a "tungsten bronze". There is a wide variety of elements which can be inserted in the A site; these include $\mathrm{Li}, \mathrm{Na}, \mathrm{Mg}, \mathrm{Al}, \mathrm{K}, \mathrm{Ca}, \mathrm{Cu}, \mathrm{Ag}, \mathrm{Tl}$, Pb, La, Ce, Pr, Nd, Sm, Eu, Gd, Tb, Dy, Ho, Er, Tm, Lu [14, 15, 102, 113]. The chemical formula for such compounds is often written as $\mathrm{M}_{x} \mathrm{WO}_{3}$, with $x$ continuously variable between 0 and 1 . In general, the maximum level of intercalation is less than 50\%, and in some cases less than 5\%. Samples with high $x$ contents are quite lustrous and exhibit metallic electronic properties [89], from which the name "tungsten bronzes" is derived. However all such compounds with $x>0$ are termed "bronzes" in literature, although they may not have metallic properties. For smaller atoms such as $\mathrm{Na}^{+}$it is possible for all sites to be occupied, i.e. $x=1$ [255]. The majority of work performed on such compounds has concentrated on alkali metal ion intercalation, with $\mathrm{Na}^{+}$in particular being the most common and the first to be studied [285]. For $\mathrm{Na}_{x} \mathrm{WO}_{3}(x>0.3)$ the cell is cubic, with a linear increase in the cell dimension observed [33] and continuous colour change with increasing $x$, from dark blue to red to golden yellow [255].

Tungsten bronze samples are often prepared by the method of Straumanis [255] which entails placing a stoichiometric mix of $\mathrm{WO}_{3}, \mathrm{Na}_{2} \mathrm{WO}_{4}$, and $\mathrm{W}$ metal inside a closed tube and firing at $950^{\circ} \mathrm{C}$. To produce samples with $x$ values close to 1 , however, an excess of $\mathrm{Na}_{2} \mathrm{WO}_{4}$ is required. Brightly coloured single crystals are formed up to $1 \mathrm{~cm}$ to a side. They can be cleaved along the crystal planes and are not readily attacked by acid or alkali solutions [31].

Even low levels of ion intercalation can cause a huge difference in the ob- 


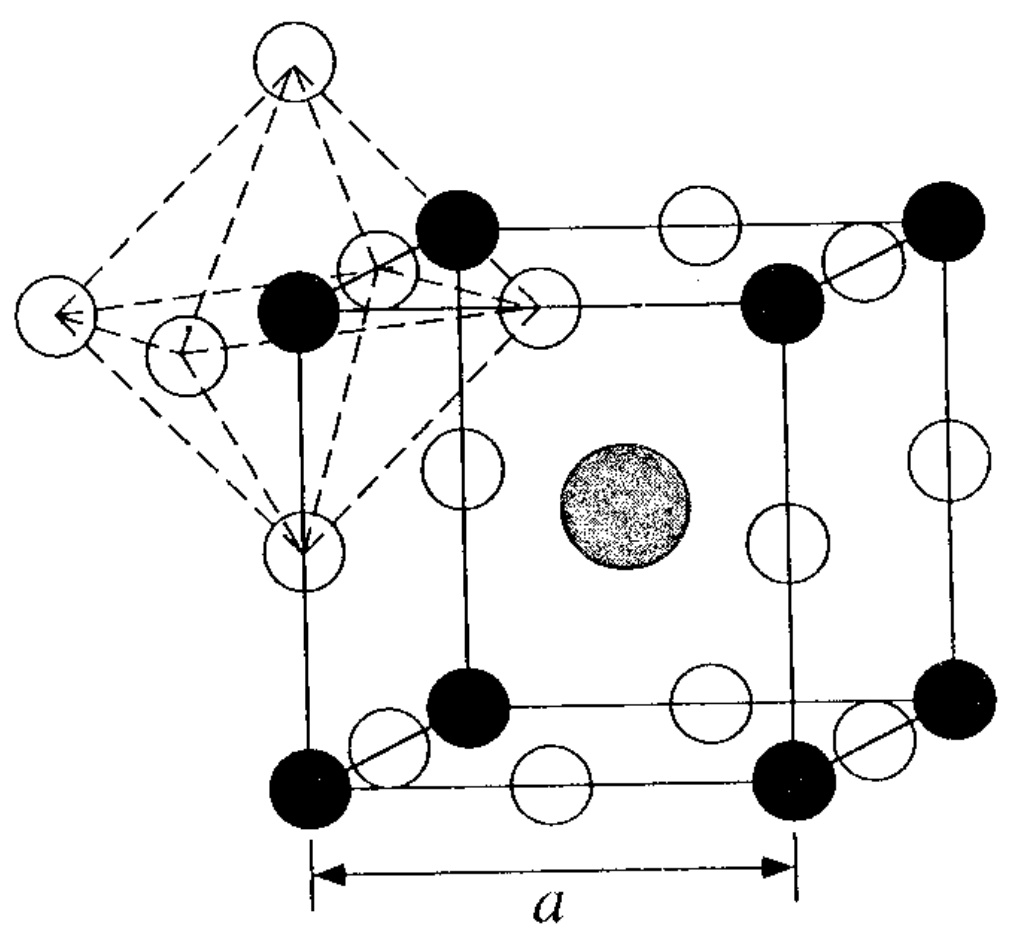

Figure 1.1: A diagram of the cubic tungsten bronze structure, where tungsten atoms are shown as black circles, oxygen as white circles, and the intercalated ion as the grey circle. The octahedral co-ordination of the B-site is shown. From [114]. 
served colour of the sample [92]. This has prompted research into switchable glazings, or "smart windows" which are quite absorbing in the 'on' state but relatively transparent in the 'off' state. Differences of 30\% to $95 \%$ transmission have been observed [1], which are completely reversible. Commercial applications normally involve ion transfer between a $\mathrm{WO}_{3}$ layer and a layer of another electrochromic material, such as $\mathrm{V}_{2} \mathrm{O}_{5}, \mathrm{Ni}(\mathrm{OH})_{2}, \mathrm{Nb}_{2} \mathrm{O}_{5}$ or $\mathrm{In}_{2} \mathrm{O}_{3}$ $[1,96]$ in a solid state reaction. The mechanism for colouring involves two reactions, e.g. [156]:

$$
\begin{array}{r}
\mathrm{Li}_{\mathrm{x}} \mathrm{V}_{2} \mathrm{O}_{5} \text { (uncoloured) } \rightarrow \mathrm{x} \cdot \mathrm{Li}^{+}+\mathrm{V}_{2} \mathrm{O}_{5} \text { (coloured) } \\
\left.\mathrm{WO}_{3} \text { (uncoloured }\right)+\mathrm{x} \cdot \mathrm{Li}^{+} \rightarrow \mathrm{Li}_{\mathrm{x}} \mathrm{WO}_{3} \text { (coloured) }
\end{array}
$$

In this case there are two colouration reactions occurring as the $\mathrm{V}_{2} \mathrm{O}_{5}$ species is depleted of $\mathrm{Li}^{+}$ions which then populate the $\mathrm{WO}_{3}$ layer.

Commercial stoichiometric tungsten trioxide powder is a pale yellow colour, which degrades in atmospheric conditions over 1-2 days to a pale green colour. This is indicative of a loss of oxygen, which can be regained by storing in an oxygen atmosphere or heating in oxygen for a few hours. An increase in oxygen partial pressure, even at room temperature, is sufficient to re-oxygenate the structure. The colouration caused by oxygen non-stoichiometry has long been studied. Thin films of tungsten trioxide deposited by chemical vapour deposition, magnetron sputtering or electronbeam evaporation are often transparent when stoichiometric but become blue-coloured as oxygen is removed [93]. Their conductivity also increases, but for the same level of colouration as a cation-inserted film, the conductivity is much less. This indicates that the mechanism for mobility is not simply due to delocalised electrons/holes but changes in the band structure near the Fermi level, which are different for the two types of material [92, 95].

Optical measurements and Raman studies on tungsten trioxide films [159, 161, 162] yield some insight into the electrochromic mechanism in these films: As cations are introduced or oxygen atoms are removed, $\mathrm{W}^{5+}$ ions are formed to maintain charge neutrality. The optical absorption is due to electron exchange between adjacent $\mathrm{W}^{5+}$ and $\mathrm{W}^{6+}$ ions. In amorphous films there 
also exist $\mathrm{W}^{4+}$ ions due to the disordered structure, which result in a higher colouration efficiency due to the additional $\mathrm{W}^{4+}-\mathrm{W}^{5+}$ transitions [160].

Stoichiometric $\mathrm{WO}_{3}$ is reported to be a semiconductor, with an indirect band gap $[19,93]$. The value of the band gap as measured on thin films varies from $2.5-3.2 \mathrm{eV}$, depending on crystallite size [225]. From X-ray photoelectron spectroscopy (XPS) it is found that the valence band lies solely on the oxygen $2 p$ states, while the conduction band comprises only tungsten $5 d$ states [19]. This is confirmed by computational methods [35, 114, 172, 209] which also show an direct gap of $0.5-1 \mathrm{eV}$ (not observed) and an indirect gap at $2-3 \mathrm{eV}$. The dielectric constant of the material is temperature dependent and changes abruptly at structural phase transitions [163]. A typical given value for the dielectric constant at room temperature is $\sim 300$ [174]. Studies of the optical properties of tungsten trioxide thin films yield an index of refraction, $n$, between 1.9-2.6, depending on thickness, crystallinity, and density. This is compared with the single crystal value for $n$ of 2.5 [68].

While the $\mathrm{ReO}_{3}$-type structured $\mathrm{WO}_{3}$ is the most stable [82], it is possible to form alternative structures under specific synthesis conditions. These all involve different arrangements of the $\mathrm{WO}_{6}$ corner-shared octahedral building blocks.

The tetragonal phase exhibits 4- and 5-sided rings, as shown in Figure 1.2 [14]; while the hexagonal phase exhibits 6-sided rings (Figure 1.3) [207]. These form in two-dimensional sheets which are stacked directly on top of each other, resulting in one-dimensional channels which run throughout the material. It is also possible to form pyrochlore structures, which are open three-dimensional structures based on 6-membered rings (Figure 1.4) [106]. As in the $\mathrm{ReO}_{3}$ structure, it is also possible to intercalate ions into the vacancies in these alternative structures to form bronzes [91, 106].

Bulk superconductivity has been observed in tetragonal sodium tungsten bronzes up to $3 \mathrm{~K}$ [243]. In cubic sodium-doped $\mathrm{WO}_{3}$ single crystals, under strict synthesis conditions it is possible for the sodium to be diffused into the surface ( $<10 \mathrm{~nm}$ depth). In such samples, granular superconductivity has been reported with a critical temperature, $T_{c}$, of around $91 \mathrm{~K}$ [167]. This appears to be a surface effect, indicating that if one were able to form a 


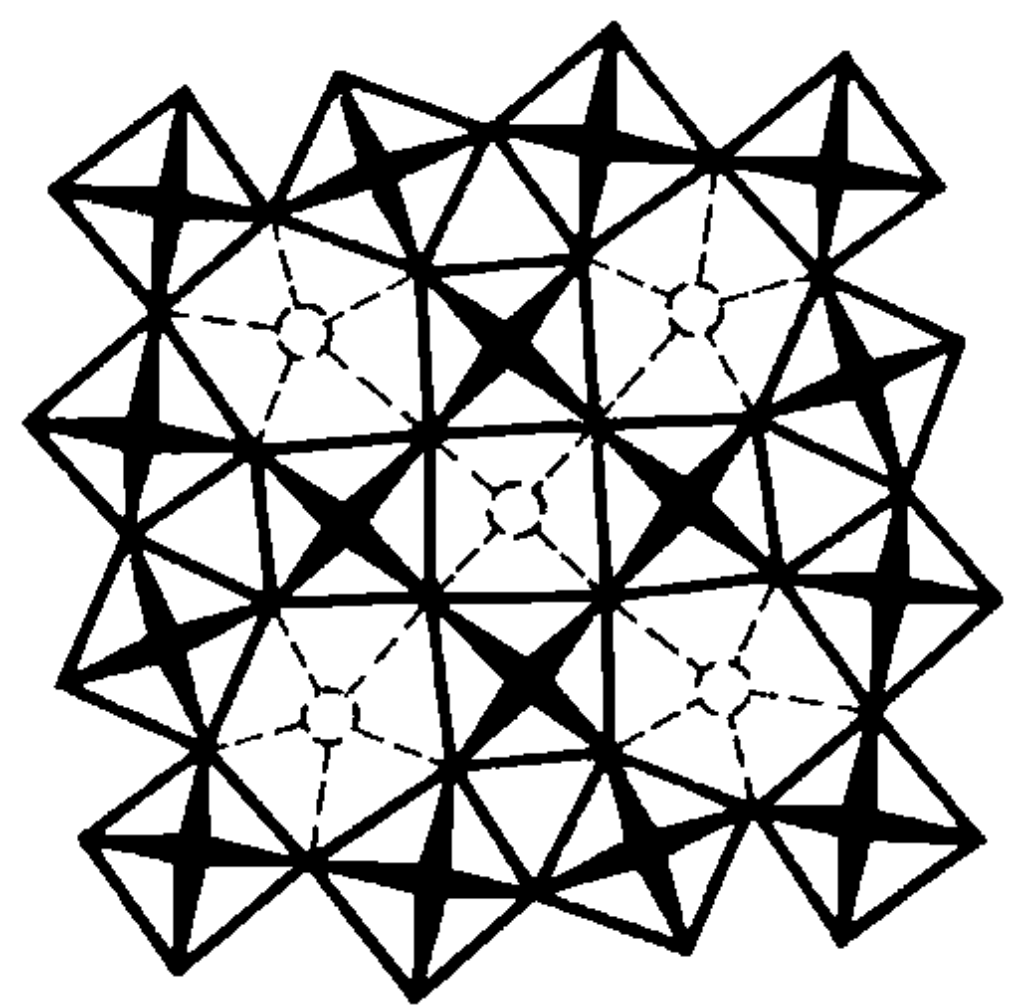

Figure 1.2: A schematic diagram of tetragonal $\mathrm{WO}_{3}$, showing how the cornershared octahedra arrange to form 4- and 5-sided tunnels in the structure. From [14]. 


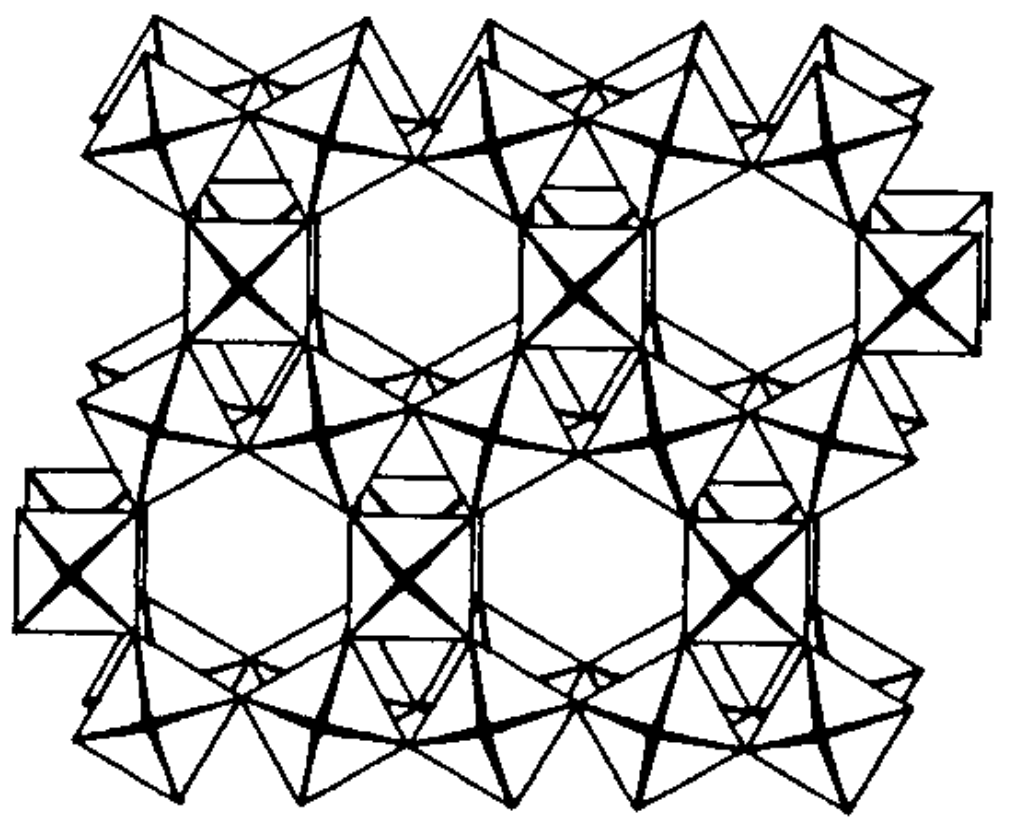

Figure 1.3: A schematic diagram of hexagonal $\mathrm{WO}_{3}$, showing the hexagonal tunnels formed by corner-shared octahedra. From [82].

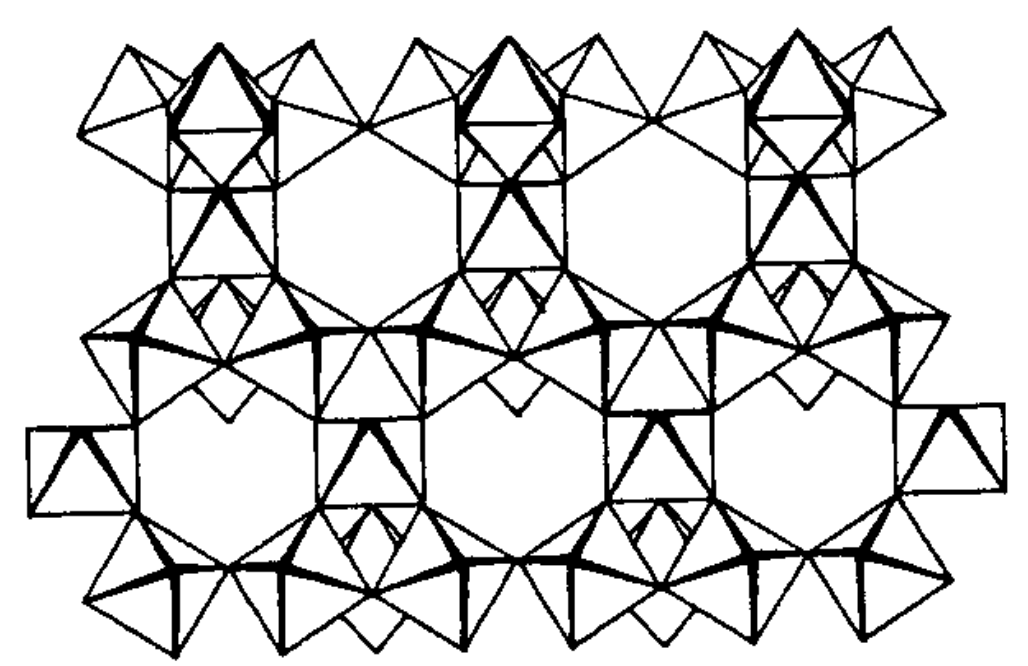

Figure 1.4: A schematic diagram of $\mathrm{WO}_{3}$ pyrochlore structure, illustrating the network of corner-shared octahedra to form 3-dimensional tunnels. From [82]. 
material with electronically decoupled two-dimensional sheets of tungsten oxide, similar interesting behaviour could perhaps be expected. Initially, this was the primary motivation for the research described in this thesis.

It is indeed possible to form layered tungsten oxide structures. Tungsten oxide hydrates, $\mathrm{WO}_{3} \cdot n \mathrm{H}_{2} \mathrm{O}$ (given the term "tungstic acids" - which may be misleading, as the hydrogen atoms do not dissociate as in a true acid), form into layers of corner-shared $\mathrm{WO}_{6}$-like octahedra, with the apical $\mathrm{W}-\mathrm{O}$ bonds alternating between $\mathrm{W}=\mathrm{O}$ and $\mathrm{W}-\mathrm{OH}_{2}$ terminations [257]. This is identical to the structure of $\mathrm{MoO}_{3} \cdot \mathrm{H}_{2} \mathrm{O}$, shown in Figure 1.5 [145]. For $n>$ 1 , the structure has additional $\mathrm{H}_{2} \mathrm{O}$ molecules situated between the layers. Both molybdenum and tungsten oxide layers have been used as the inorganic component of hybrid materials before $[13,99,125]$, but not extensively. This may be due in part to the formation of heteropolytungstate and -molybdate ions at low pH [134]. Several other molybdenum oxide-organic hybrids have been reported $[69,70,157,288]$ but the inorganic frameworks are not simple corner-shared arrangements of the $\mathrm{MoO}_{6}$ octahedra. For this reason, the scope of this work will concentrate mainly on tungsten oxide hybrids that do exhibit this structure.

The quite considerable variety of structure and function of the tungsten oxide system can be extended even further by the intercalation of organic molecules. This is the subject of this thesis, as described in the following chapters.

\subsection{Thesis outline}

Chapter 2 describes all of the experimental techniques and equipment used for the experimental work undertaken in this thesis.

Chapter 3 details the synthesis and initial structural characterisation of the 'parent' tungsten oxide hybrid system. A comprehensive understanding of the structure and behaviour of the simplest system is necessary before continuing on to more complex hybrid systems, with more complex organic molecules, adjusting the inorganic layer, or both. Several synthesis methods are compared using x-ray diffraction, electron microscopy, and infrared 


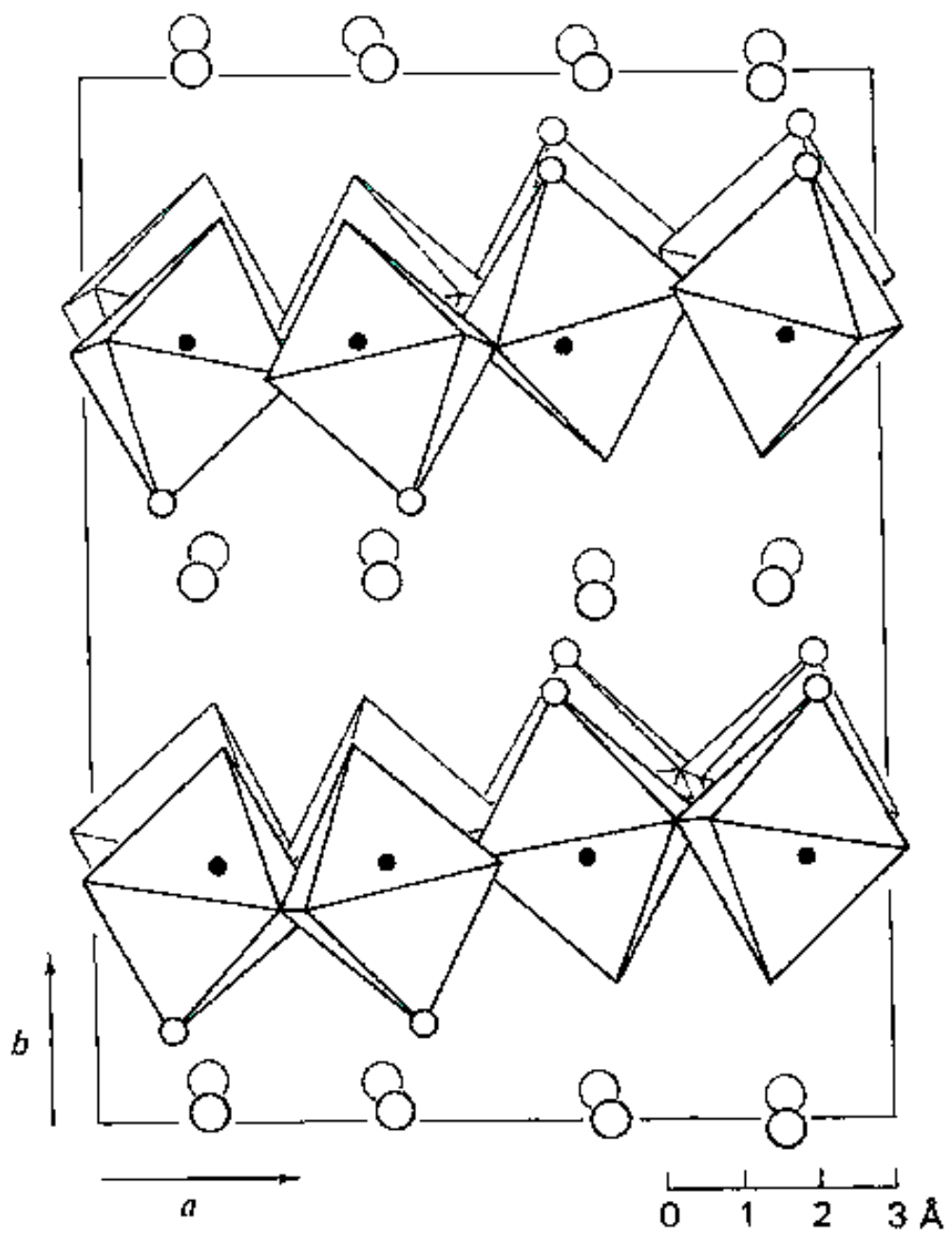

Figure 1.5: The structure of $\mathrm{WO}_{3} \cdot n \mathrm{H}_{2} \mathrm{O}$, where $n=2$. From [145]. 
spectroscopy. These techniques, along with elemental microanalysis, thermogravimetric analysis, and Raman spectroscopy are used to describe the layered structure, chemical composition, and bonding arrangement of the hybrid materials and how these vary systematically across the series of organic molecules intercalated.

When surmising how these materials might behave upon doping, it is useful to study a known, related system. Chapter 4 presents ab initio calculations of a number of tungsten oxide-based systems. An initial examination of a previously well-studied system (both computationally and experimentally), the alkali tungsten bronzes $\left(\mathrm{M}_{x} \mathrm{WO}_{3}\right)$, is provided. This looks at electronic and structural trends across the doping range and also confirms the validity of the computations, which agree well with previous studies. This work is then extended to another doped tungsten oxide system which has been littlestudied computationally, oxygen-deficient $\mathrm{WO}_{3-x}$. Given this background, computations of some experimentally simple (although computationally complex) tungsten oxide hybrids were performed. The electronic band structures of the materials were compared with experimental UV-visible spectroscopy results and are in good agreement. Thus, Chapter 3 provides the structural and composition studies of the 'parent' tungsten oxide hybrids, and Chapter 4 discusses their electronic properties through computation and experiment.

Chapter 5 expounds upon Chapter 3 through the addition of transition metal species to the inorganic layer in an attempt to dope the inorganic layer via substitution. Each transition metal ion series forms a different structure, and again, a variety of techniques was used to determine structure, chemical composition, and bonding arrangement of the resulting hybrids. The compounds formed display a wide variety of interesting properties, including magnetism, which is explored in more detail for each material in Chapter 6 .

Chapter 7 provides a brief exploration into an area of research which is still in progress at the time of writing, that of using electrochemical techniques to attempt to intercalate small metal ions into the hybrid structures. Again, the known systems of $\mathrm{WO}_{3}$ and $\mathrm{H}_{2} \mathrm{WO}_{4}$ are used as comparatives.

This thesis is hardly an exhaustive treatise on tungsten oxide-based organicinorganic hybrid materials. There are thousands more individual compounds 
in hundreds of chemical series that could be studied, and several other techniques not readily available to us that could be used for characterisation. Chapter 8 provides some examples of future work that could be conducted, followed by the final conclusions. 


\section{Chapter 2}

\section{Experimental}

This chapter outlines the synthesis techniques that have been used to form the tungsten oxide hybrid materials and the various methods used to characterise them and measure their properties, used throughout this thesis.

\subsection{Synthesis of tungsten oxide-based hybrids}

Three main synthetic techniques were used to form the tungsten oxide hybrids $[48,49]$ :

- Solution based method (evaporation of solvent),

- 'Melt method' (intercalation via pure liquid amine),

- Chimie douce ('soft chemistry', soaking).

Each of these will be described in detail. We consider first the simple tungsten oxide-based hybrid materials and discuss later the incorporation of transition metal ions (Section 2.2).

The following chemicals were used in the syntheses with no further purification:

Tungstic acid, $\mathrm{H}_{2} \mathrm{WO}_{4}, 99 \%$, Aldrich

Ammonia solution, 30 wt. $\%, \mathrm{BDH}$

1,2-diaminoethane (DA2), $\mathrm{H}_{2} \mathrm{~N}\left(\mathrm{CH}_{2}\right)_{2} \mathrm{NH}_{2}, 99 \%$, BDH GPR

1,4-diaminobutane (DA4), $\mathrm{H}_{2} \mathrm{~N}\left(\mathrm{CH}_{2}\right)_{4} \mathrm{NH}_{2}, 99 \%$, Aldrich 
1,6-diaminohexane (DA6), $\mathrm{H}_{2} \mathrm{~N}\left(\mathrm{CH}_{2}\right)_{6} \mathrm{NH}_{2}, 98 \%$, Sigma

1,8-diaminooctane (DA8), $\mathrm{H}_{2} \mathrm{~N}\left(\mathrm{CH}_{2}\right)_{8} \mathrm{NH}_{2}, 98 \%$, Aldrich

1,12-diaminododecane (DA12), $\mathrm{H}_{2} \mathrm{~N}\left(\mathrm{CH}_{2}\right)_{12} \mathrm{NH}_{2}, 98 \%$, Aldrich

methylamine (MA1), $\mathrm{H}_{2} \mathrm{NCH}_{3}, 25-30 \%$ in $\mathrm{H}_{2} \mathrm{O}, \mathrm{BDH}$

ethylamine (MA2), $\mathrm{H}_{2} \mathrm{NCH}_{2} \mathrm{CH}_{3}, 70 \%$ in $\mathrm{H}_{2} \mathrm{O}$, Aldrich

butylamine (MA4), $\mathrm{H}_{2} \mathrm{~N}\left(\mathrm{CH}_{2}\right)_{3} \mathrm{CH}_{3}, 99.5 \%$, Aldrich

hexylamine (MA6), $\mathrm{H}_{2} \mathrm{~N}\left(\mathrm{CH}_{2}\right)_{6} \mathrm{CH}_{3}, 99 \%$, Aldrich

dodecylamine (MA12), $\mathrm{H}_{2} \mathrm{~N}\left(\mathrm{CH}_{2}\right){ }_{11} \mathrm{CH}_{3}, 98 \%$, Aldrich

aniline, $\mathrm{C}_{6} \mathrm{H}_{5} \mathrm{NH}_{2}, 99 \%$, Aldrich

benzylamine, $\left(\mathrm{C}_{6} \mathrm{H}_{5}\right) \mathrm{CH}_{2} \mathrm{NH}_{2}, 99 \%$, Aldrich

phenethylamine (phen), $\left(\mathrm{C}_{6} \mathrm{H}_{5}\right)\left(\mathrm{CH}_{2}\right)_{2} \mathrm{NH}_{2}, 99.5 \%$, Aldrich

p-phenylenediamine/1,4-diaminobenzene (DAphen), $\mathrm{H}_{2} \mathrm{~N}\left(\mathrm{C}_{6} \mathrm{H}_{4}\right) \mathrm{NH}_{2}, 97 \%$, Aldrich

2-amino-benzylamine, $\mathrm{H}_{2} \mathrm{~N}\left(\mathrm{C}_{6} \mathrm{H}_{4}\right) \mathrm{CH}_{2} \mathrm{NH}_{2}, 98 \%$, Aldrich

\subsubsection{Solution-based method}

Tungstic acid, $\mathrm{H}_{2} \mathrm{WO}_{4}$, was dissolved in aqueous ammonia solution with heating and stirring at $70-80^{\circ} \mathrm{C}$ for approximately 6 hours. In a separate beaker, a 2-fold molar excess of the desired organic was dissolved in an appropriate solvent (see Table 2.1) with heating, and added to the hot tungstate solution. The temperature was maintained at $70-80^{\circ} \mathrm{C}$ and as the solvent was evaporated under nitrogen flow, a white-cream coloured powder formed. In the latter stages of evaporation the flask was transferred to a water bath to ensure even heating. The product was washed and filtered with ethanol and dried in air at $80^{\circ} \mathrm{C}$ overnight. It is possible to remove the saturated solution from the heat before a precipitate forms, and use this to spin- or dip-coat films for applications.

\subsection{2 'Melt method'}

Most simple aliphatic (straight-chain) and aromatic amines melt cleanly at temperatures in the range of -50 to $200^{\circ} \mathrm{C}$ (i.e. using a bench-top labora- 


\begin{tabular}{ll}
\hline Organic Intercalate & Solvent used \\
\hline \hline DA2 & distilled $\mathrm{H}_{2} \mathrm{O}$, ammonia solution, none \\
DA4 & ammonia solution \\
DA6 & distilled $\mathrm{H}_{2} \mathrm{O}$, ammonia solution \\
DA8 & ammonia solution \\
DA12 & ethanol \\
MA4 & heptane \\
MA6 & heptane \\
benzylamine & ethanol \\
phenethylamine & ethanol \\
2-amino-benzylamine & ethanol \\
\hline
\end{tabular}

Table 2.1: Solvents used to dissolve organic reagents

tory hot-plate). An excess of amine was melted and tungstic acid powder added, followed quickly by a few drops of ammonia solution, with vigorous stirring. An immediate colour change from yellow to white-cream was observed. The product was washed and filtered with ethanol and dried in air at $80^{\circ} \mathrm{C}$ overnight.

\subsubsection{Chimie douce method}

Utilising the pre-existing layered structure of $\mathrm{H}_{2} \mathrm{WO}_{4}$, it is possible to apply chimie douce methods to intercalate organic molecules between the $\mathrm{WO}_{4}$ layers.

Tungstic acid was stirred at room temperature for a period of days to weeks in an appropriate solution of the organic amine ${ }^{1}$. The solvent was chosen so that it dissolved the amine at room temperature, but not the tungstic acid nor the hybrid product. As the reaction progressed the colour of the powder was observed to change from yellow to white. The product was filtered, washed with ethanol, and dried in air at $80^{\circ} \mathrm{C}$ overnight.

\subsubsection{Thin film preparation}

Thin films of $\mathrm{WO}_{3}$ were deposited on quartz substrates $1-1.5 \mathrm{~cm}$ to a side via three methods:

\footnotetext{
${ }^{1}$ Toluene for DA6, alcohol for the others.
} 
- Spin-coating of $\mathrm{H}_{2} \mathrm{WO}_{4}$ dissolved in aqueous ammonia solution, ethanolamine, or a mixture of the two, followed by annealing at $550-600^{\circ} \mathrm{C}$ in $\mathrm{O}_{2}$ for 1 hour;

- Sputtering of $\mathrm{W}$ metal films, subsequently heated in $\mathrm{O}_{2}$ at $450^{\circ} \mathrm{C}$ for 6 days;

- Electron-beam deposition of $\mathrm{WO}_{3}$.

Thin films of the hybrid materials were spin-coated from a saturated solution of $\mathrm{H}_{2} \mathrm{WO}_{4}$ in aqueous ammonia with the organic amine added. This was obtained by following the same procedure for making the hybrid powders but halting the reaction before a precipitate forms. The normal procedure is to form the hybrid powder and then redissolve in a suitable solvent [130]. However we have been unable to find a solvent which completely dissolves the hybrid, and from which the layered phase can be recrystallised. This is not surprising given the low solubility of $\mathrm{WO}_{3}$ and $\mathrm{H}_{2} \mathrm{WO}_{4}$ in almost any solvent.

\section{$2.2 \quad$ Hybrids with transition metals}

As in Section 2.1, three methods were used to synthesise the transition metal tungstate hybrids:

- Solution based method (evaporation of solvent),

- 'Melt method' (intercalation via pure liquid amine),

- Chimie douce ('soft chemistry', soaking).

In addition to the chemicals listed in Section 2.1, the following were also used in the syntheses (with no further purification):

$\mathrm{Na}_{2} \mathrm{WO}_{4} \cdot 2 \mathrm{H}_{2} \mathrm{O}, 99 \%$ Ajax Chemicals

$\mathrm{MnCl}_{2} \cdot 4 \mathrm{H}_{2} \mathrm{O}, 99.0 \%$ BDH AnalaR

$\mathrm{FeCl}_{3}, 98 \%$ Ajax Chemicals

$\mathrm{Fe}\left(\mathrm{NO}_{3}\right)_{3} \cdot 9 \mathrm{H}_{2} \mathrm{O}, 99 \%$ BDH AnalaR 
$\mathrm{Co}\left(\mathrm{NO}_{3}\right)_{2} \cdot 6 \mathrm{H}_{2} \mathrm{O}, 99 \%$ Acros

$\mathrm{NiSO}_{4} \cdot 6 \mathrm{H}_{2} \mathrm{O}, 98 \%$ Aldrich

$\mathrm{CuCl}_{2}, 97 \%$ Aldrich

Manganese and iron tungstate hybrids could be made successfully via the solution method as follows:

The tungsten and organic reagents were prepared in hot $\left(80^{\circ} \mathrm{C}\right)$ aqueous ammonia solution as per Section 2.1.1. Nitrogen gas was bubbled at a moderate rate through the stirred solution as a cold (room-temperature) aqueous solution of the manganese or iron salt was quickly added. A precipitate immediately formed that darkened with time. While the initial precipitates were of the desired phase, the best quality products with greatest crystallinity were obtained by evaporating the rest of the solvent as per the tungsten oxide hybrids, with the latter stages of evaporation performed on a water bath. The use of nitrogen gas is essential to prevent the formation of oxide species. The products were filtered and washed with ethanol and dried under vacuum overnight.

When cobalt, nickel, or copper solutions were added to the solution in the above manner, they formed complexes with the ammonia rather than hybrid materials. However, hybrids of copper and cobalt tungstate with DA2 as the organic intercalate have been formed via a solution technique, using $\mathrm{Na}_{2} \mathrm{WO}_{4}$ as the source of tungstate ions, and all reagents dissolved in water. This is indicated in Table 5.1. However, as we shall see in Section 5.2.1 the (M,W)-DA2 compound for $\mathrm{M}=\mathrm{Co}, \mathrm{Ni}$ and $\mathrm{Cu}$ appears to form a completely different structure. Syntheses of (Co,W)-DA6, (Ni,W)-DA6 and $(\mathrm{Cu}, \mathrm{W})-\mathrm{DA} 6$ from $\mathrm{Na}_{2} \mathrm{WO}_{4}$ aqueous solution were unsuccessful. Hence the 'melt' and chimie douce methods were used more extensively for transition metal hybrids with $\mathrm{Co}, \mathrm{Ni}$ and $\mathrm{Cu}$ in particular.

As the 'melt method' and chimie douce method both require a layered structure to directly intercalate the organic molecules, and tungsten oxide hydrate was used for the W-DAn hybrids formed by these methods, we elected to form the hydrates of the metal tungstates to use as the inorganic reagent in these syntheses. 
Transition metal tungstate hydrates, $\mathrm{MWO}_{4} \cdot n \mathrm{H}_{2} \mathrm{O}$, form as precipitates for most metal ions $\mathrm{M}^{2+}$ when an aqueous $\mathrm{M}^{2+}$ solution is added to an aqueous tungstate (normally $\mathrm{Na}_{2} \mathrm{WO}_{4}$ ) solution at room temperature [170]. The precipitates were filtered and washed thoroughly with distilled water to remove excess starting materials and $\mathrm{NaCl}$, then dried under vacuum overnight. They were then used as-is in either the melt or chimie douce method to produce the hybrids. A comparison of these methods is given in Section 5.1.1.

\subsection{Preparation of hybrids for electrochemi- cal doping}

$\mathrm{WO}_{3}, \mathrm{H}_{2} \mathrm{WO}_{4}$, and hybrid material powders were mixed in a 1:6 - 1:8 ratio with an organic polymeric binder (polypropylene carbonate, 'qpac-40', dissolved in cyclohexanone) to form a paste, which was then coated onto a gold foil working electrode $1 \mathrm{~cm}$ to a side.

The electrolyte consisted of a $0.5 \mathrm{M}$ solution of $\mathrm{NaCl}$ in distilled water. Immediately before each experiment the solution was de-oxygenated by bubbling nitrogen gas through for at least ten minutes. $\mathrm{Ag} / \mathrm{AgCl}$ was used as the reference electrode.

Ramp rates of $10-50 \mathrm{mV} \cdot \mathrm{s}^{-1}$ were used over a voltage range of $-400-$ $+400 \mathrm{mV}$. The voltage range is limited by the oxidation and reduction of the $\mathrm{H}_{2} \mathrm{O}$ solvent.

While many samples exhibited an intense colour change (from pale yellow to dark blue), there was not enough material to measure their diffuse reflectance. X-ray diffraction was used to observe any phase changes or lattice expansion due to the incorporation of the additional ions.

\subsection{Measurement techniques and parameters}

The various experimental procedures used in the course of the project are outlined as follows: 


\section{X-ray Diffraction}

X-ray diffraction (XRD) was used as a primary characterisation technique. To avoid preferential alignment of the grains, samples were prepared in the following manner: A small amount of powder was lightly ground and placed on a custom-made quartz slide holder with negligible XRD background along with 5-10 drops of ethanol to make a slurry. The powder particles were spread evenly over the holder and the ethanol allowed to evaporate. Alternatively a small amount of grease was smeared onto the quartz holder and the ground powder sprinkled onto it.

Diffraction patterns were obtained using a Philips PW1700 series powder diffractometer employing Co K $\alpha$ radiation $(\lambda=1.789 \AA)$. Typical parameters for initial characterisation of the powders are an angle range of $4-80^{\circ} 2 \theta$ with total scan time 50 minutes.

\section{Scanning Electron Microscopy}

Scanning electron microscopy (SEM) was performed on powder samples that had been coated with carbon prior to viewing. SEM micrographs were obtained using a Leo 440 microscope with up to 50,000 times magnification capability, with an Oxford ISIS energy dispersive X-ray analysis (EDX) system attached.

\section{Transmission Electron Microscopy}

Transmission electron microscopy (TEM) was undertaken in both imaging and diffraction modes on two separate instruments: a Phillips EM400 with tungsten filament operated at $120 \mathrm{kV}$, and a JEOL 2011 high-resolution instrument with a $\mathrm{LaB}_{6}$ filament operated at $200 \mathrm{kV}$. Samples were lightly ground with a liberal amount of isopropanol with a mortar and pestle, and a suspension of fine particles transferred using a dropper to a standard carbon film supported on a copper grid. 


\section{Microanalysis}

Samples of 5-10 mg were sent to the Campbell Microanalytical Laboratory at Otago University [301] for elemental analysis of C, H and N, using a Carlo Erba Elemental Analyser EA1108. In each case a minimum of two samples were analysed. The absolute experimental uncertainties are estimated from the range of results (given as percentages by weight of the total mass) and are typically $<0.05 \%$ for $\mathrm{C}$ and $\mathrm{N}$ and $<0.1 \%$ for $\mathrm{H}$.

\section{Thermogravimetric analysis}

Thermogravimetric analysis (TGA) was performed on 10-15 mg of sample using a Rheometrics STA1500 TG-DTG/DSC thermogravimetric analyser over temperature ranges up to $800^{\circ} \mathrm{C}$ with a heating rate of $5^{\circ} \mathrm{C} / \mathrm{min}$ under flowing nitrogen gas.

\section{Infrared spectroscopy}

Samples were prepared for infrared measurements using the KBr disc method: $10 \mathrm{mg}$ of sample was incorporated with $0.10-0.15 \mathrm{~g} \mathrm{KBr}$, ground thoroughly using a mortar and pestle, and pressed into discs of $15 \mathrm{~mm}$ diameter at a pressure of $10 \mathrm{MPa}$. The samples, $\mathrm{KBr}$, and pressed discs were kept in a dry box at all times to minimise adsorption of atmospheric water. Infrared spectra were collected on a Bomem DA8 FT spectrometer over the mid-IR range (450-4000 $\left.\mathrm{cm}^{-1}\right)$ with a resolution of $2 \mathrm{~cm}^{-1}$.

\section{Raman spectroscopy}

Samples were prepared for Raman measurements by pressing 8-10 mm pellets ( $0.2 \mathrm{~g}$ ) of ground powder at pressures of 5-15 MPa. Raman spectra were collected using a Jobin-Yvon LabRam HR spectrometer with an excitation wavelength of $632.8 \mathrm{~nm}$ and resolution of $4 \mathrm{~cm}^{-1}$.

\section{UV-visible spectroscopy}

UV-visible spectroscopy was performed on powder samples using a diffuse reflectance integrating sphere, and on thin films using a simultaneous transmit- 
tance and reflectance set-up. Data were collected using a GCA/McPherson 2051 monochromate spectrometer over the range $250-1200 \mathrm{~nm}$ with spectral bandwidth of $1 \mathrm{~nm}$.

Finely-ground powder samples were packed in a custom-made cell with a quartz wedged window $\sim 20 \mathrm{~mm}$ in diameter.

For UV-visible thin film transmittance measurements, films must be deposited on quartz substrates as these are transparent over the wavelength studied.

\section{XANES and EXAFS}

Two manganese tungstate hybrids, (Mn,W)-DA2 and (Mn,W)-DA6, and $\mathrm{MnWO}_{4} \cdot n \mathrm{H}_{2} \mathrm{O}$ were sent to the National Synchrotron Radiation Research Centre in Taiwan for x-ray absorption near edge spectroscopy (XANES) and extended x-ray absorption fine structure (EXAFS) measurements. Spectra were obtained at both the Mn K-edge and W L3-edge in the hope of determining the local environment around each manganese and tungsten atom in the structure. Fits were performed using the FEFF and FEFFIT analysis programs.

\section{Magnetic susceptibility}

A Quantum Design MPMS XL SQUID magnetometer was used to perform various magnetisation measurements on selected materials. The instrument can measure samples in DC fields of up to $7 \mathrm{~T}$ and temperatures from $2-$ $300 \mathrm{~K}$, with a sensitivity of $10^{-8} \mathrm{emu}$. Powder samples were ground and 5-20 mg tightly sealed in a gelatin capsule with negligible magnetic susceptibility. Both field-cooled and zero-field cooled temperature sweeps were performed at constant applied fields. AC susceptibility was measured at frequencies of 30-900 $\mathrm{Hz}$ and an amplitude of 3 Oe (although the instrument frequency range is up to $0.01-1000 \mathrm{~Hz}$ and amplitudes up to 4 Oe). 


\section{Chapter 3}

\section{Composition and Basic Structure}

This chapter compares the various synthesis techniques that have been used to form the tungsten oxide hybrid materials, and discusses the results of the basic structural analysis using various techniques.

\subsection{Synthesis}

As described in Chapter 2, tungsten oxide-based hybrid materials were synthesised by three main synthetic techniques: the solution-based, 'melt' and chimie douce methods. While the solution method was preferred, it was not possible to form some of the hybrids by this method and so other techniques were explored.

First we compare the products formed by each of three methods, and then study the characterisation results.

\subsubsection{Comparison of synthesis methods}

In the present studies, the solution-based evaporation of solvent method has been the most extensively used of the three techniques, as indicated in Table 3.1. Precipitation of a product from solution generally forms larger crystals than a quicker method, as the crystals nucleate relatively slowly in the saturated solution. 
Chimie douce on the other hand preserves the layered structure, and for this reason has been used to synthesise hybrids where a solution-based method would destroy the inorganic layers (e.g. $\mathrm{MnO}_{2}$ birnessite and swelled perovskites, see Appendix A). However, the particle size of the product is limited by the particle size of the inorganic ingredient.

There have been reports of certain metal halide hybrids being prepared from a melt or by a solid state reaction [199, 202]. The 'melt method' is expected to give the lowest quality samples for several reasons. When the $\mathrm{H}_{2} \mathrm{WO}_{4}$ powder is added to the melt, the mixture quickly solidifies and hence the incorporation of the organic molecules within the layered structure is hindered. The whole reaction is very quick, of the order of minutes. There is no solvent present for the dissolution and recrystallising of the product, and in short the reaction is very inhomogeneous. It is therefore quite surprising that not only is the same product formed as in the other two methods, but there are no traces of the starting material $\mathrm{H}_{2} \mathrm{WO}_{4}$. The hybrid structure must therefore be quite structurally stable and diffusion rates for the intercalant quite fast.

Figure 3.1 illustrates the difference in XRD between three samples of W-DA6 formed by each of the three methods presented here, obtained as described in Section 2.4.

As can clearly be seen, the signal-to-noise ratio of the sample produced by the solution-based method is much greater than those by the melt and chimie douce methods. The line widths are fairly similar in this instance, however as will be seen in Chapter 5, the line widths of the samples prepared by the solution-based method are normally much narrower than the other two, indicating better crystallinity. Other variations in line intensity, seen in Figure 3.1, can be due to grain alignment which may intensify some reflections (e.g. $00 \ell)$.

Figure 3.2 shows SEM micrographs of the same three samples (a, solution; c, melt; and d, chimie douce). These were obtained as described in Section 2.4. Figure $3.2 \mathrm{~b}$ is another sample formed by the solution method with methylamine $\left(\mathrm{H}_{2} \mathrm{NCH}_{3}\right)$ added as a structural templating agent. (While the 


\begin{tabular}{|l|l|l|l|}
\hline Organic interca- & Solution & Melt & Chimie douce \\
late & & & \\
\hline DA2 & $\sqrt{ }$ & - & $\sqrt{ }$ \\
DA4 & $\sqrt{ }$ & $\sqrt{ }$ & - \\
DA6 & $\sqrt{ }$ & - \\
DA8 & $\sqrt{ }$ & - & $\sqrt{ }$ \\
DA12 & $\times$ & - & $\times$ \\
MA1 & $\times$ & $\sqrt{ }$ \\
MA2 & $\sqrt{ }$ & - \\
MA4 & $\sqrt{ }$ & - \\
MA6 & $\times$ & - & - \\
MA12 & $\times$ & - \\
aniline & $\sqrt{ }$ & $\sqrt{ }$ \\
benzylamine & $\sqrt{ }$ & $\sqrt{ }$ \\
phen & $\times$ & - & - \\
DAphen & $\sqrt{ }$ & - & $\sqrt{ }$ \\
2-amino- & & - & \\
benzylamine & & & \\
\hline
\end{tabular}

Table 3.1: Synthesis methods used for various organic intercalates. $\sqrt{ }=$ successful, $\times=$ unsuccessful, $-=$ not attempted.

${ }^{1}$ with heating.

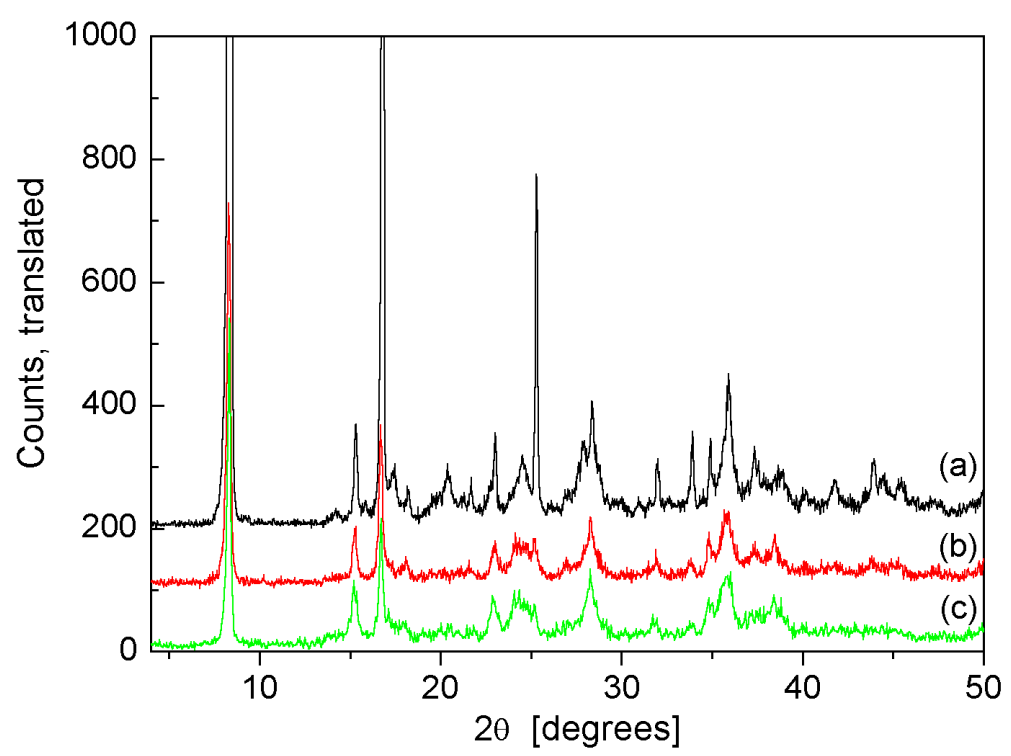

Figure 3.1: XRD patterns of three samples synthesised by (a) solution, (b) melt, and (c) chimie douce methods. The solution method pattern has been truncated; the maximum value is 10400 on the scale. 


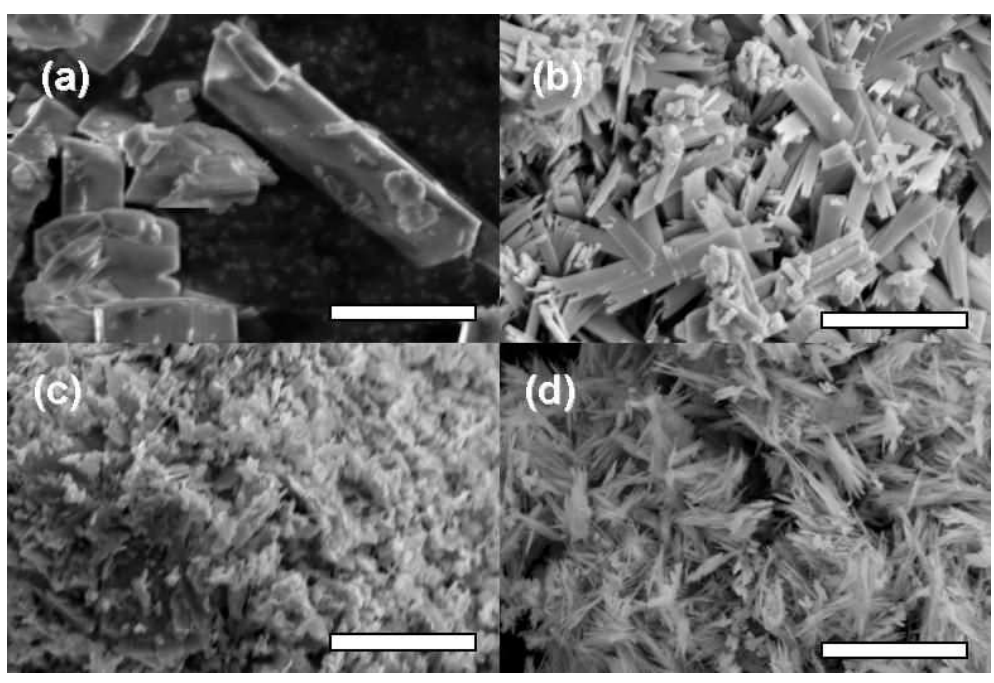

Figure 3.2: SEM micrographs of W-DA6 samples synthesised by (a,b) solution, (c) melt, and (d) chimie douce methods. The scale bars shown correspond to $10 \mu \mathrm{m}$ in each case.

morphology is quite different from the sample shown in Figure 3.2a, XRD confirms the same crystal phase has been formed, and microanalysis indicates the methylamine is not present in the final structure.)

The solution-based method produces well-defined crystals of various sizes (Figure 3.2a) which can be quite platy under slightly different synthesis conditions (Figure 3.2b). The chimie douce method produces crystals of a similar size and morphology to the starting inorganic material, as expected, because the inorganic material is not dissolved and recrystallised. The 'melt' method produces a material with visually the lowest crystallinity of the three methods, with a high degree of disorder due to the rapid formation of the hybrid.

Despite this, the linewidths in the XRD patterns (a measure of crystallinity) are fairly similar. This is probably due to the sample preparation for XRD requiring grinding of the sample, and the larger crystallites are broken up. Manual grinding can reduce powder crystallites to $1-2 \mu \mathrm{m}$ in size, and it is quite conceivable that in the melt method there are crystallites of this size. 


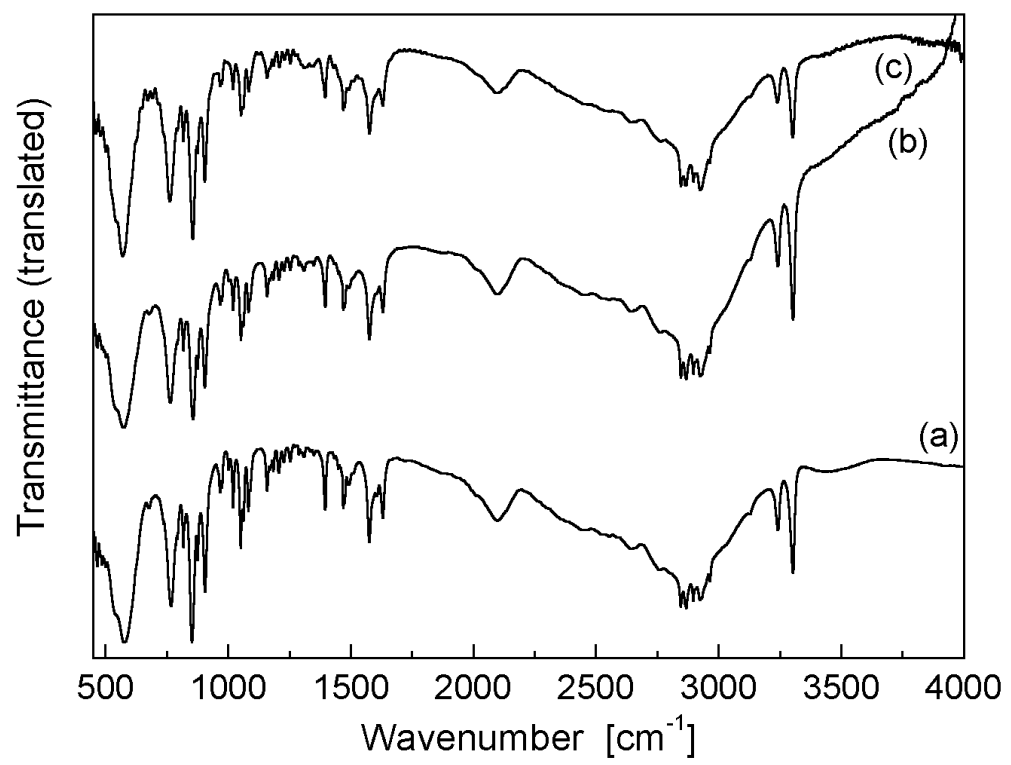

Figure 3.3: IR spectra of W-DA6 samples produced by (a) solution-based method, (b) 'melt' method, and (c)chimie douce method.

Figure 3.3 shows the IR spectra of the same three samples. While a more detailed IR study is given later in Section 3.3, the point to note here is the consistency between the three spectra corresponding to the three synthesis techniques: the peaks do not change in shape or position.

These three methods were used quite extensively in the initial characterisation of the tungsten oxide (and later, transition metal tungstate) hybrid samples. SEM gives a visual indication of morphology, and in the transition metal tungstates EDX can be used at the same time to determine the transition metal to tungsten ratio. XRD gives a semi-quantitative measure of phase purity and crystallinity. While IR is not affected by the quality of the sample in general (provided it is of a single phase), it gives additional structural information (as elaborated in Section 3.3). 


\subsection{2 $\mathrm{pH}$ dependence}

Early in this study an examination of $\mathrm{pH}$ dependence in the synthesis of the hybrids was undertaken. ${ }^{1}$ Considering first the metal halide hybrids, these are synthesised in acid solutions of the halide (i.e. $\mathrm{HCl}, \mathrm{HBr}, \mathrm{HI}$ ), which protonates the amines and provides the extra halide ions to convert the $\mathrm{MX}_{2}$ starting material to $\mathrm{MX}_{4}$ layers [196, 200]. The protonation of the amines is important to retain charge balance, i.e.

$$
\mathrm{MX}_{2}+\mathrm{H}_{2} \mathrm{~N}\left(\mathrm{CH}_{2}\right)_{\mathrm{n}} \mathrm{NH}_{2}+2 \mathrm{HX} \rightarrow \mathrm{MX}_{4}{ }^{2-} \cdot\left[\mathrm{H}_{3} \mathrm{~N}\left(\mathrm{CH}_{2}\right)_{\mathrm{n}} \mathrm{NH}_{3}\right]^{2+}
$$

Slow evaporation, unseeded cooling and seeded cooling were three methods used to grow crystals of the metal halide hybrids of sizes up to $\sim 10 \mathrm{~mm}$ on a side [6].

$\mathrm{H}_{2} \mathrm{WO}_{4}$ is soluble in alkaline solution and $\mathrm{HF}$, with aqueous ammonia solution the most common solvent (and used here). The $\mathrm{pH}$ was altered with $\mathrm{HCl}, \mathrm{HNO}_{3}$ or acetic acid and hybrid syntheses were attempted. At $\mathrm{pH} 6$ crystals were formed that were large enough to perform single crystal x-ray diffraction. It was found that instead of a tungsten oxide layered hybrid compound, a hybrid based on $\left[\mathrm{W}_{12} \mathrm{O}_{42}\right]^{12-}$ Keggin ion clusters was formed [86]. Such hybrids have also been reported by other groups, at $\mathrm{pH}$ values ranging from 2-8 [84, 124].

While the low $\mathrm{pH}$ protonates the amine species, it also forms $\mathrm{W}-\mathrm{OH}$ terminations on the soluble $\mathrm{WO}_{4}{ }^{2-}$ ions. These terminations preferentially lead to the formation of polyoxotungstate cluster ions below $\mathrm{pH} \sim 8[134$, 177].

Therefore, to avoid the formation of the polyoxotungstate ions, a high $\mathrm{pH}$ $(\sim 10)$ must be maintained. This is achieved in the solution-based method with excess ammonia solution required to dissolve $\mathrm{H}_{2} \mathrm{WO}_{4}$. Aqueous ammonia solution boils at $\sim 80^{\circ} \mathrm{C}$, which is less than the boiling point of water $\left(100^{\circ} \mathrm{C}\right)$. Even so, during the stage where most of the ammonia is removed and only water is being evaporated, the presence of aqueous ammonium hydroxide and the organic amines maintain an alkaline $\mathrm{pH}$ of $\sim 9.5$ [27].

\footnotetext{
${ }^{1}$ This work was carried out by Natalie Robinson, Hana Robson Marsden and David Bitauld as a student project.
} 


\section{$3.2 \quad$ Structure}

As discussed in the previous section, different microstructures can be formed from tungstate solutions by varying the $\mathrm{pH}$ - the most noticeable of these being the formation of polyoxotungstate cluster ions.

These cluster ions can also be formed in solutions containing organic amine species which are unable to form layered hybrids due to steric hindrance $^{2}$. This situation was encountered with several aromatic based hybrids where the terminal $-\mathrm{NH}_{2}$ group is directly adjacent to the benzene ring (e.g. aniline and DAphen) [87]. It is possible to synthesise copper halide hybrids with such organic molecules, due to copper displaying a large distortion range arising from the Jahn-Teller effect [180, 196, 283]. While distortions exist in layered $\mathrm{WO}_{3} \cdot n \mathrm{H}_{2} \mathrm{O}$, they are not large enough to allow the accommodation of the bulky aromatic rings, due to steric hindrance by the terminal oxygens. Aromatic molecules with the amine separated from the ring by at least one $-\mathrm{CH}_{2}-$ linkage (e.g. benzylamine, phenethylamine) are able to form hybrids with tungsten oxide.

\subsubsection{X-ray diffraction}

X-ray diffraction (XRD) has been the primary method of characterising the hybrid materials, and is detailed in Section 2.4. Figure 3.4a shows typical XRD patterns for the W-DAn series $(n=2,4,6,8,12)$. These display a series of $00 \ell$ lines, including the intense 001 peak at low $2 \theta$ angles. This is characteristic of layered structures and the $d$-spacing given by the lines corresponds to the interlayer spacing. This is shown in Figure 3.4b plotted versus the length of the organic diamine spacer molecule.

From the gradient and intercept of this line we can determine some basic features of the structure. A linear fit gives the equation $d=1.26 n+4.77$, where $d$ is the interlayer spacing and $n$ denotes the number of carbon atoms in the alkyl chain.

The $\mathrm{C}-\mathrm{C}$ bond length is $1.54 \AA$ and bond angle $109.4^{\circ}[12]$, giving a

\footnotetext{
${ }^{2}$ While hydrothermal synthesis can force such sterically hindered molecules into the tungsten oxide layers, this has not been pursued in the project to date.
} 

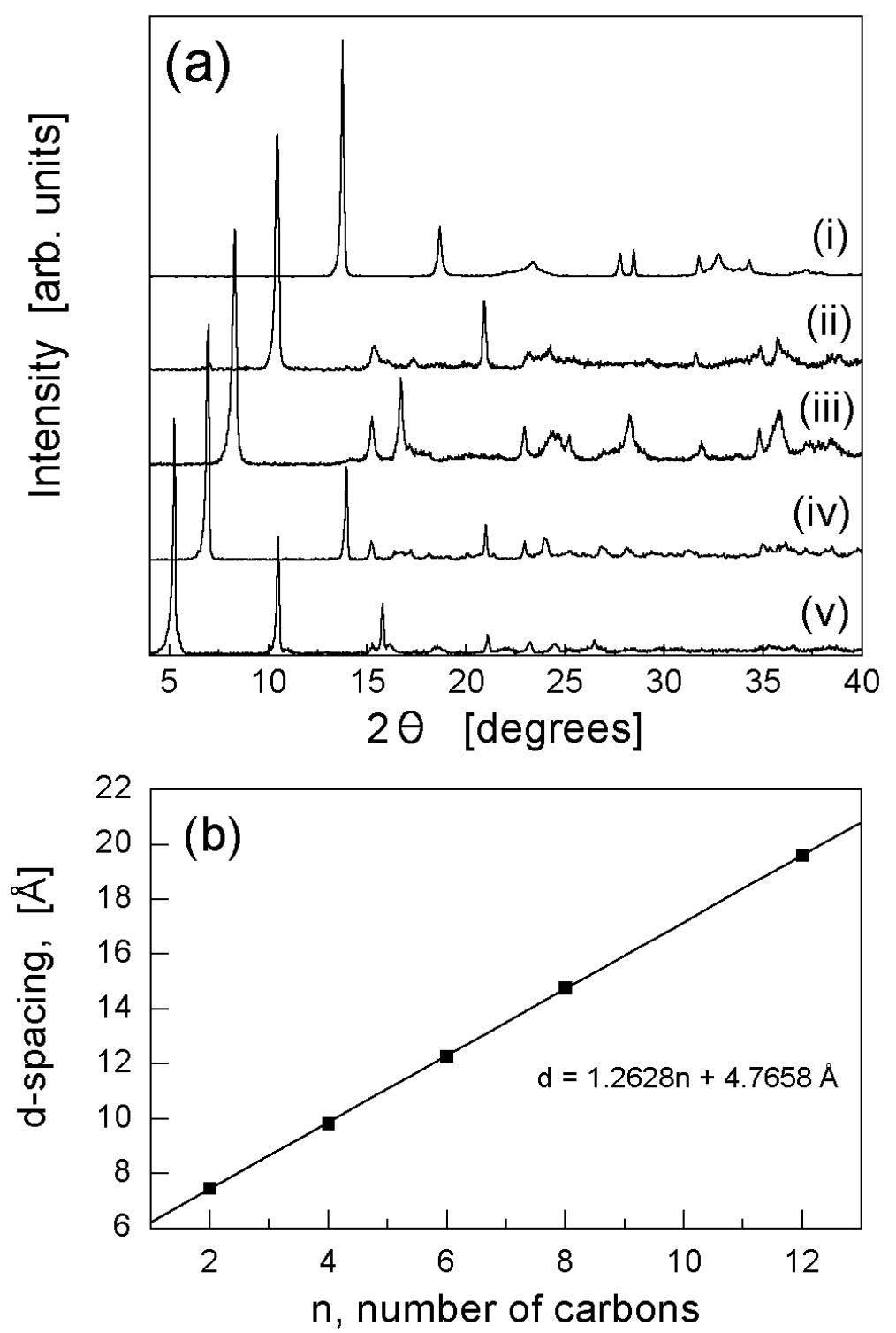

Figure 3.4: a) XRD patterns of W-DAn, (i) $\mathrm{n}=2$, (ii) $\mathrm{n}=4$, (iii) $\mathrm{n}=6$, (iv) $\mathrm{n}=8$, (v) $\mathrm{n}=12$. b) Plot indicating the $d$-spacing as determined from the positions of the $00 \ell$ lines. 


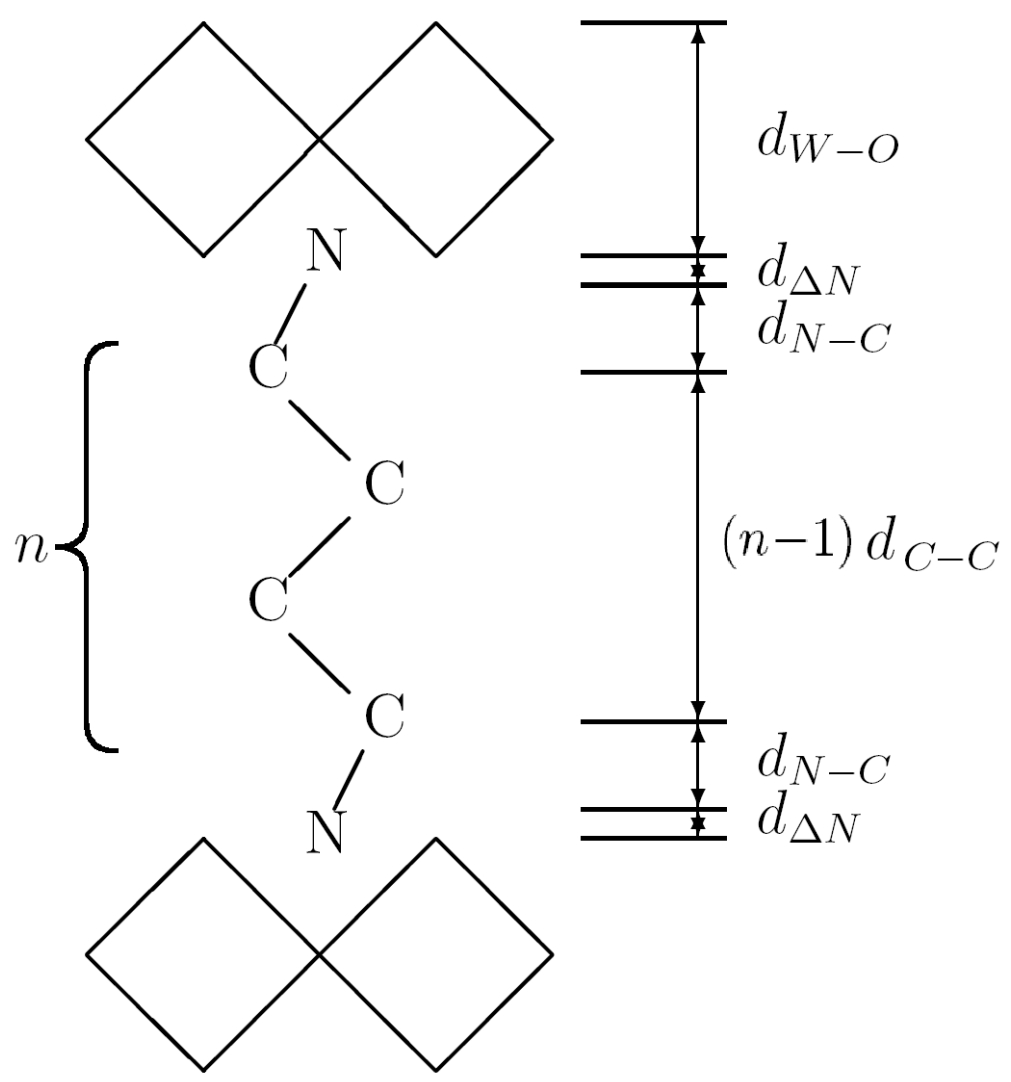

Figure 3.5: Diagram of W-DAn structure.

lengthwise component of $1.26 \AA$ per carbon atom in the chain. The gradient of the line is $1.26 \AA$ per carbon and hence the organic molecules lie perpendicular to the inorganic layers.

From the intercept we can calculate the inorganic layer spacing. There is no physical system corresponding to a zero carbon chain; rather the expected length is given by

$$
d=d_{W-O}+2 d_{\Delta N}+2 d_{N-C}+(n-1) d_{C-C}
$$

as shown in Figure 3.5. Thus when $n \rightarrow 0$, the expression becomes

$$
d=d_{W-O}+2 d_{\Delta N}+2 d_{N-C}-d_{C-C}
$$

Substituting $d=4.77, d_{N-C}=1.47 \cos \left(109^{\circ} / 2\right)$ and $d_{C-C}=1.26 \AA$, we obtain 


$$
d_{W-O}+2 d_{\Delta N}=3.56 \AA
$$

In $\mathrm{WO}_{3}$, the $\mathrm{W}-\mathrm{O}$ layer is $3.74 \AA[262]$ and in $\mathrm{H}_{2} \mathrm{WO}_{4}$ the $\mathrm{W}-\mathrm{O}$ layer is $4.03 \AA$ [257]. $d_{\Delta N}$ is expected to be very small (i.e. the nitrogen atoms of the ammonium groups lying in plane with the apical oxygen atoms) or even negative. Thus the XRD results indicate that for the W-DAn system, there is exactly one $\mathrm{WO}_{4}$ unit per inorganic layer and the organic 'spacer' molecules lie perpendicularly to the inorganic layers. This is also found to be the case in Section 3.2.3.

\subsubsection{Electron microscopy}

SEM images shown in Figure 3.2 (obtained as detailed in Section 2.4) demonstrate that the largest crystals are formed via the evaporation of solvent method. The largest crystals are $\sim 20 \times 10 \times 5 \mu \mathrm{m}$ in size, rendering them too small for conventional single-crystal x-ray diffraction to elucidate their structure. Attempts to measure the diffraction from these microcrystals using a synchrotron source (via the SCrAPS programme) were also fruitless. Powder XRD data does not give enough information for Rietveld refinement without being confident of the unit cell obtained. Powder samples were also sent for neutron diffraction measurements, however the hydrogen atoms were too highly scattering to provide useful data. At the time of writing, fully deuterated forms of the hybrids were being prepared for additional neutron powder diffraction experiments. In the absence of comprehensive X-ray and neutron diffraction data, we performed selected area electron diffraction (SAED) using a transmission electron microscope (TEM), as described in Section 2.4 .

Typical SAED patterns for W-DA2 and W-DA6 are shown in Figure 3.6. These are reasoned to be directly related to the in-plane lattice parameters, because of the crystal morphology (flat plates) and the similarity between the two photographs - there is no evidence of lattice expansion in one direction due to the longer DA6 instead of DA2 organic 'spacer' molecule. (The $c$-axis 

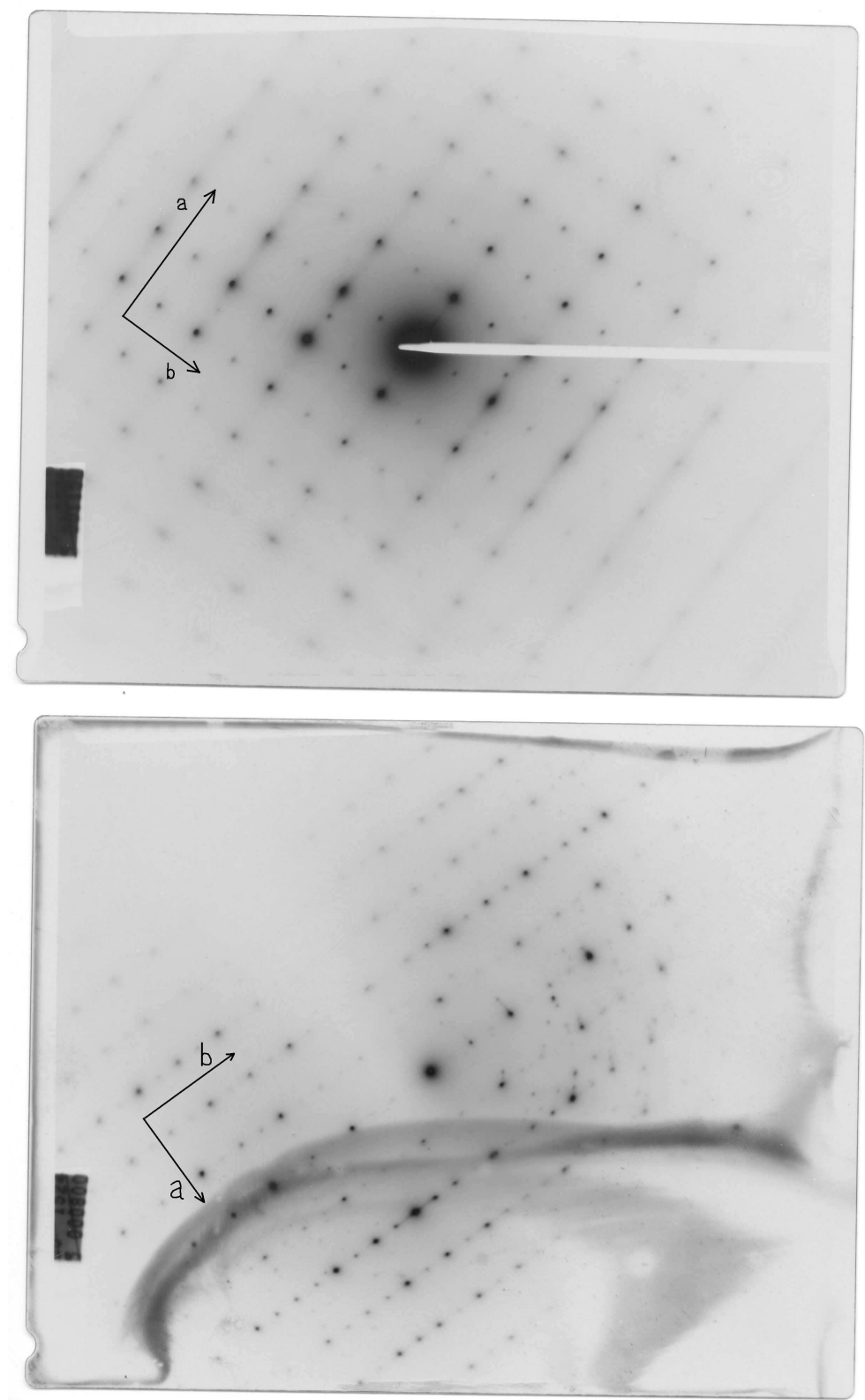

Figure 3.6: SAED patterns of W-DA2 (top) and W-DA6 (bottom), showing the $a b$-plane. 


\begin{tabular}{|c|ccc|c|c|c|}
\hline Sample & $\% \mathrm{C}$ & $\% \mathrm{H}$ & $\% \mathrm{~N}$ & Formula & Expect & I:O ratio \\
\hline W-DA2 & 8.38 & 2.69 & 9.63 & $\mathrm{C}_{2} \mathrm{H}_{7.64} \mathrm{~N}_{1.97}$ & $\mathrm{C}_{2} \mathrm{H}_{8-10} \mathrm{~N}_{2}$ & $1: 0.917$ \\
W-DA6 & 20.19 & 4.53 & 7.74 & $\mathrm{C}_{6} \mathrm{H}_{16.10} \mathrm{~N}_{1.98}$ & $\mathrm{C}_{6} \mathrm{H}_{16-18} \mathrm{~N}_{2}$ & $1: 0.977$ \\
\hline
\end{tabular}

Table 3.2: Microanalytical results for W-DAn hybrids showing mass percentages, observed and expected stoichiometries, and the inorganic:organic ratio.

lengths of W-DA2 and W-DA6, from XRD (Section 3.2.1) are $7.45 \AA$ and $12.28 \AA$ respectively.)

Knowing the parameters used in these photographs by calibration with standard samples, we can calculate the two axes shown to be $a=5.62$ and $b=7.33 \AA \pm 10 \%$ for W-DA2 and $a=11.46$ and $b=5.35 \AA \pm 10 \%$ for W-DA6. While these results are not as accurate as one might have hoped, it does give us the ratio between the two axes (1.305 and 2.143) and the angle between them $\left(89.4^{\circ}\right.$ and $\left.88.3^{\circ}\right)$, which can be searched for in the list of possible cells from the initial stages of powder XRD refinement. This task is made difficult because of the likely possibility of supercells arising from the tendency of tungsten oxide species to form a distorted layer structure $[35,82,257]$. In W-DA2 there is evidence for the presence of a supercell by the presence of weaker spots between the more intense spots in the shorter lattice direction (spots further apart). In W-DA6 the most intense spots form an almost square shape, but along one of these directions there are weaker spots which indicate a supercell, doubled in the b-direction.

\subsubsection{Microanalysis}

Elemental microanalysis is a technique employed regularly in the characterisation of the hybrid materials to determine the composition of the organic species and the organic:inorganic ratio. These measurements were performed as detailed in Section 2.4. Typical results for the W-DAn hybrids are shown in Table 3.2, where the tungsten oxide is assumed to be present as $\mathrm{WO}_{4}$. They confirm an essentially stoichiometric composition consistent with the presumed two-dimensional layered structure. 


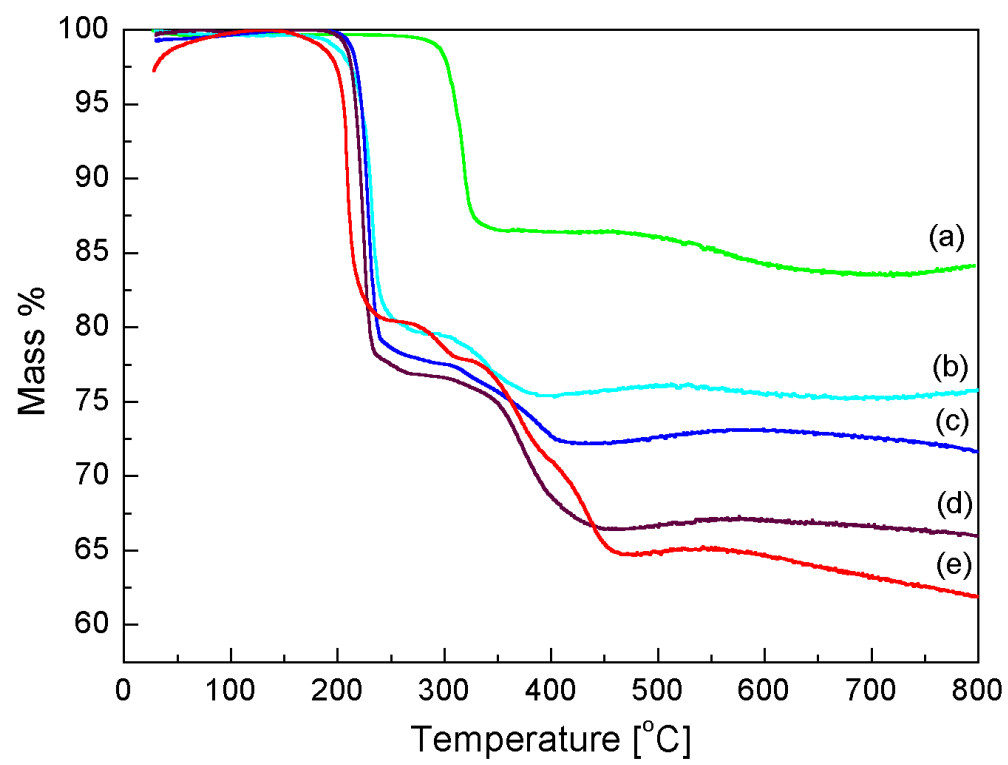

Figure 3.7: TGA scans for W-DAn, $\mathrm{n}=$ (a) 2, (b) 4, (c) 6, (d) 8 and (e) 12 .

\subsubsection{Thermogravimetric analysis}

Thermogravimetric analysis (TGA) was performed as detailed in Section 2.4. The results are shown for the W-DAn hybrid series in Figure 3.7. In each case there are two distinct mass losses at temperatures of around 230 and $370^{\circ} \mathrm{C}$ for the samples with $n>2$, and 315 and $500^{\circ} \mathrm{C}$ for the $n=2$ sample. These correspond respectively to the clean deintercalation of the organic molecules and subsequent decomposition to $\mathrm{WO}_{3}$. As shown in Table 3.3, these temperatures are much higher than the melting points (and for the shorter molecules, boiling points) of the organic molecules alone, indicating that when the organic molecules are intercalated into an inorganic structure they interact with the oxide structure more strongly than with themselves, but inversely with respect to the alkane chain length.

W-DA2 displays quite different behaviour from the other hybrid samples, as evidenced by a number of different experiments. The difference is most obvious in the TGA data above, but from TGA alone no explanation can be given. In the IR spectra of W-DA2 compared with the other W-DAn samples (as we will see in the next section), there is evidence to suggest that this sam- 


\begin{tabular}{|l|l|l|l|}
\hline$n$ & $\begin{array}{l}\text { DAn melting } \\
\text { point }\left({ }^{\circ} \mathrm{C}\right)\end{array}$ & $\begin{array}{l}\text { DAn boiling point } \\
\left({ }^{\circ} \mathrm{C}\right)\end{array}$ & $\begin{array}{l}\text { W-DAn deintercalat- } \\
\text { ion temperature }\left({ }^{\circ} \mathrm{C}\right)\end{array}$ \\
\hline 2 & 8.5 & 116.5 & 314 \\
4 & 27 & 158 & 230 \\
6 & 41 & 204 & 226 \\
8 & 51 & 225 & 221 \\
12 & 70 & $2766^{1}$ & 210 \\
\hline
\end{tabular}

Table 3.3: Melting points, boiling points and decomposition temperatures for DAn and W-DAn.

${ }^{1}$ Estimated value.

ple has a different bonding mechanism. Analysis of the modes corresponding to protonated alkyl-ammonium $\left(\mathrm{R}-\mathrm{NH}_{3}{ }^{+}\right)$and neutral alkyl-amine $\left(\mathrm{R}-\mathrm{NH}_{2}\right)$ groups indicates that while the other hybrids display both of these, the organic molecules in W-DA2 have only neutral amine terminations. This is also evident in the microanalysis (page 46) as the number of hydrogen atoms is lower relative to the number expected, compared with W-DA6 for example. However, this appears to be the only immediately obvious structural difference across the W-DAn series.

\subsection{Infrared and Raman spectroscopy}

Samples of $\mathrm{WO}_{3}, \mathrm{H}_{2} \mathrm{WO}_{4}$, W-DAn $(n=2,4,6,8,12)$, W-MAn $(n=2,4,6)$ and $\mathrm{W}$-phen were prepared and measured as outlined in Section 2.4.

\subsection{1 $\mathrm{WO}_{3}, \mathrm{H}_{2} \mathrm{WO}_{4}$ and $\mathrm{W}$-DAn samples}

The normalised infrared and Raman spectra of the solid samples studied are shown in Figures 3.8 and 3.9 respectively. The peak positions are tabulated for the region $1000-4000 \mathrm{~cm}^{-1}$ in Table 3.4 and for $0-1000 \mathrm{~cm}^{-1}$ in Table 3.5. The diaminoalkane hybrids W-DAn with alkyl lengths greater than two carbons have virtually identical spectra $[48,120]$ and so are summarised in the tables as W-DAn, in comparison with the differing W-DA2.

The infrared spectra of $\mathrm{WO}_{3}$ and its hydrate, $\mathrm{H}_{2} \mathrm{WO}_{4}$, have much fewer and broader peaks than their hybrid counterparts, due to the absence of 
modes corresponding to the organic component. Both the infrared and Raman spectra of $\mathrm{WO}_{3}$ and $\mathrm{H}_{2} \mathrm{WO}_{4}$ correspond well with those presented by Daniel et al. [66]. The results will be presented and discussed in three sections: the effect that the presence of the organic molecule has on the inorganic layer, the effect of this incorporation on the organic species, and the organic-inorganic bonding nature.

\section{The effect on the inorganic layer}

The presence of co-ordinated water molecules in $\mathrm{H}_{2} \mathrm{WO}_{4}$ can be seen by the broad O-H stretching peak at 3410 (3370) $\mathrm{cm}^{-1}$ (IR and Raman respectively), the $\mathrm{H}_{2} \mathrm{O}$ bending peak at $1614 \mathrm{~cm}^{-1}$ (IR only), and the $\mathrm{W}-\mathrm{OH}_{2}$ co-ordinated water peak in the Raman spectrum at $377 \mathrm{~cm}^{-1}$. These peaks do not occur in the $\mathrm{WO}_{3}$ spectra, as expected.

As mentioned in Section 1.3 (page 21), the structure of $\mathrm{H}_{2} \mathrm{WO}_{4}$ consists of layers of corner-shared $\mathrm{WO}_{6}$ octahedra with alternate apical arrangements of $\mathrm{W}-\mathrm{OH}_{2}$ and $\mathrm{W}=\mathrm{O}$. In the Raman spectrum the $\mathrm{W}=\mathrm{O}$ bonding is shown clearly by a sharp band centred at about $950 \mathrm{~cm}^{-1}$. This is also present in the IR spectrum. Surprisingly, the $\mathrm{WO}_{3}$ sample also exhibits a small peak at this position in both the IR and Raman spectra. This is not expected as the structure of $\mathrm{WO}_{3}$ consists only of single $\mathrm{W}-\mathrm{O}$ bonds. However it can be explained by the presence of disorder in the sample and loss of oxygen (as noted in Chapter 4), which results in the formation of a small fraction of $\mathrm{W}=\mathrm{O}$ bonds $[162]$ and a loss of inversion symmetry. There are also $\mathrm{W}=\mathrm{O}$ terminations on the surfaces of the powder particles. (Commercial $\mathrm{WO}_{3}$ powder is a pale yellow colour, which is reduced over 1-2 days in air to a pale green colour. This is indicative of a loss of oxygen but can be regained by storing in an oxygen atmosphere or heating in oxygen for a few hours.)

In the diaminoalkane hybrid compounds the characteristic $\mathrm{W}=\mathrm{O}$ Raman peak, formerly at $950 \mathrm{~cm}^{-1}$ in $\mathrm{H}_{2} \mathrm{WO}_{4}$, shifts to a lower frequency of 890-900 $\mathrm{cm}^{-1}$. While in several of these compounds there are peaks at $950 \mathrm{~cm}^{-1}$ they are not as intense as the $\mathrm{W}=\mathrm{O}$ peak. It is also of interest to note that the peak at $888 \mathrm{~cm}^{-1}$ in the W-DA2 Raman spectrum is a doublet. There may be more than one variant of the $\mathrm{W}=\mathrm{O}$ bond within the structure, as is the 


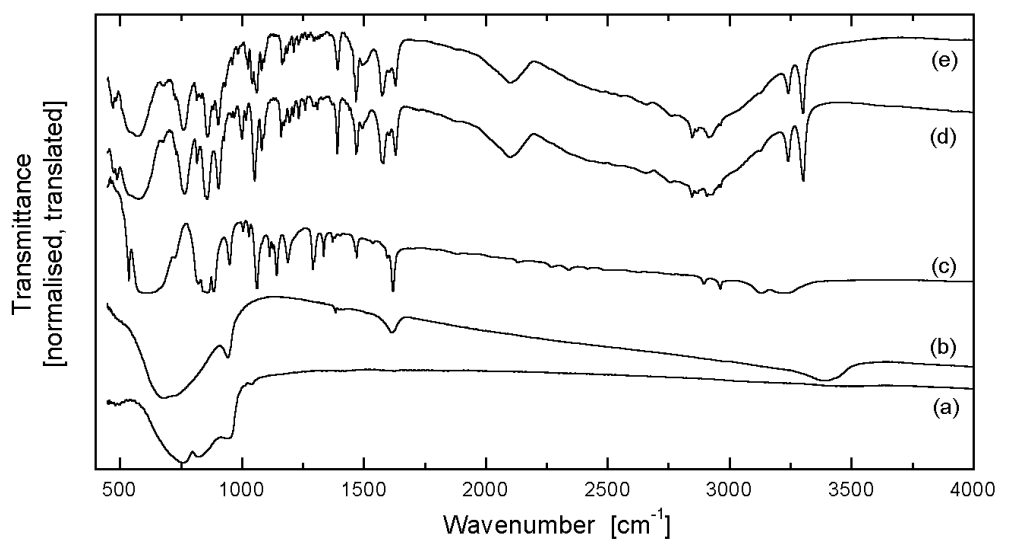

Figure 3.8: Infrared spectra of tungsten oxide-related materials: (a) $\mathrm{WO}_{3}$; (b) $\mathrm{H}_{2} \mathrm{WO}_{4}$; (c) W-DA2; (d) W-DA6; (e) W-DA12.

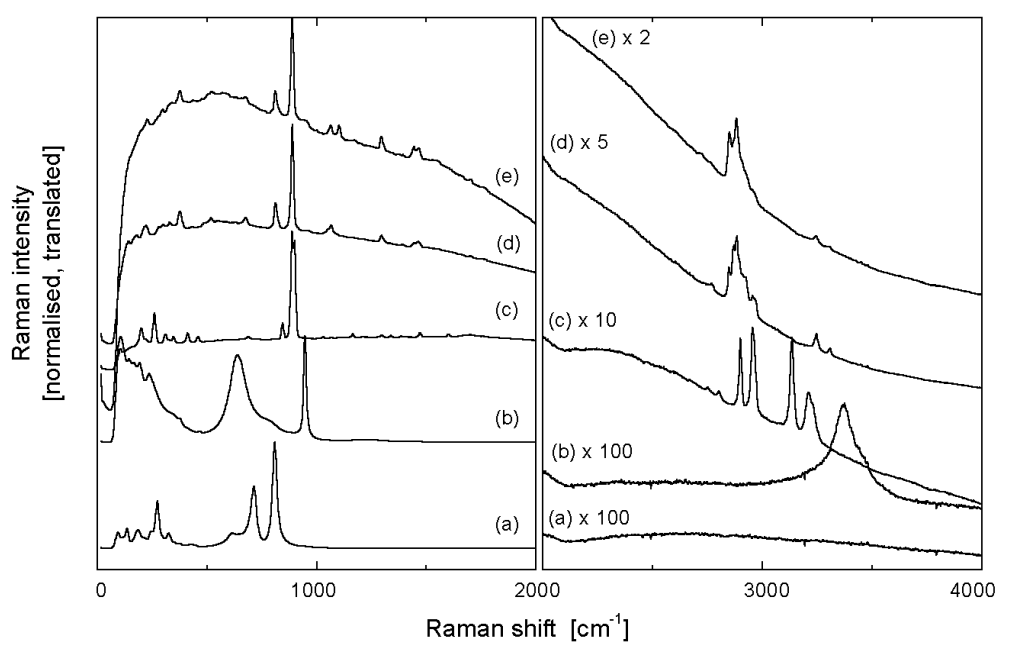

Figure 3.9: Raman spectra of tungsten oxide-related materials: (a) $\mathrm{WO}_{3}$; (b) $\mathrm{H}_{2} \mathrm{WO}_{4}$; (c) W-DA2; (d) W-DA6; (e) W-DA12. The high frequency region is displayed on a different scale to enable the important features to be seen. 


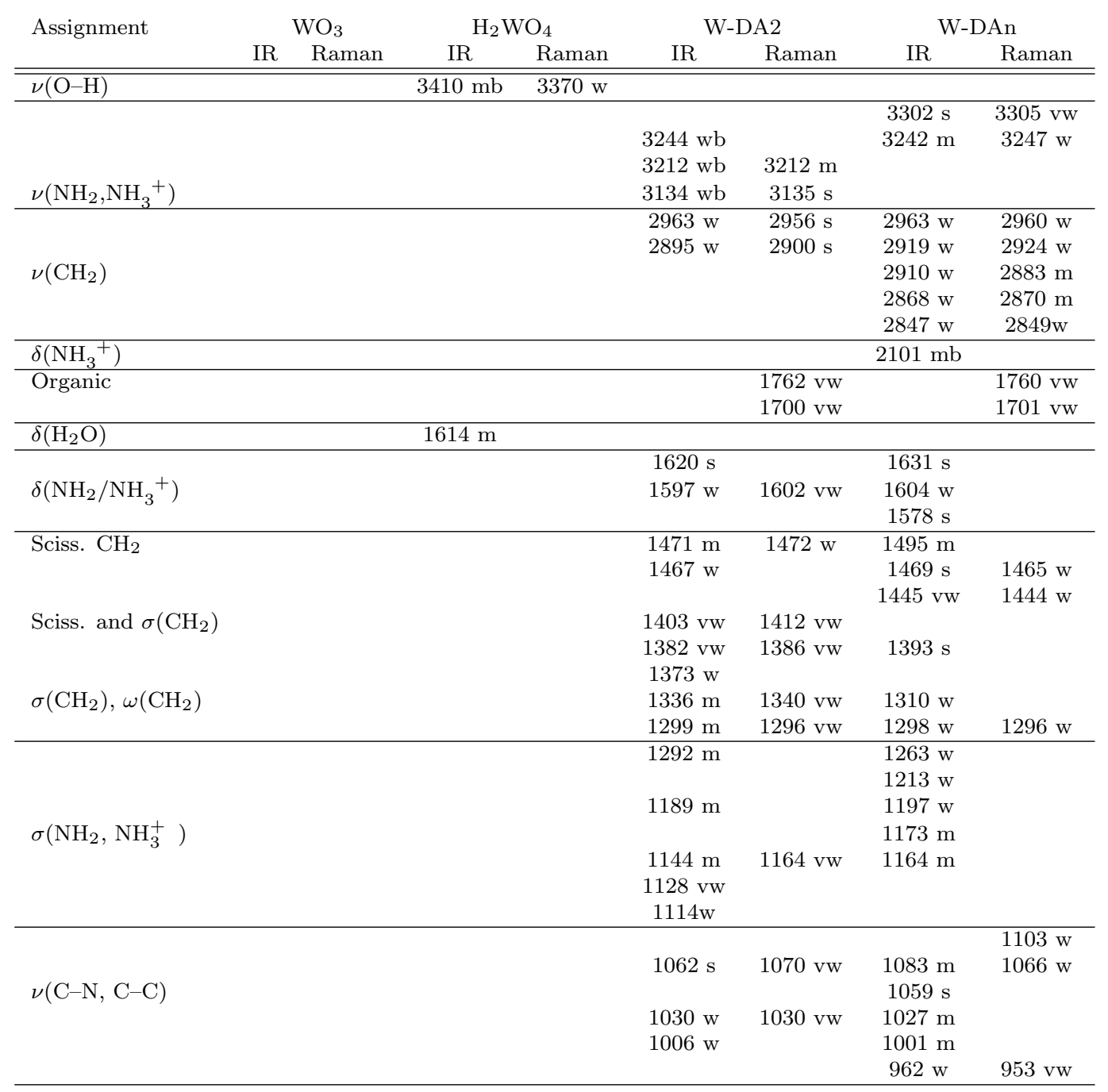

Table 3.4: Observed peaks in powder samples, $1000-4000 \mathrm{~cm}^{-1} . \nu$ stretching, $\delta$ deformation/in-plane bending, sciss. scissoring, $\sigma$ bending, $\omega$ wagging; s strong, m medium, w weak, vw very weak, b broad. 


\begin{tabular}{|c|c|c|c|c|c|c|c|c|}
\hline \multirow[t]{2}{*}{ Assignment } & \multicolumn{2}{|c|}{$\mathrm{WO}_{3}$} & \multicolumn{2}{|c|}{$\mathrm{H}_{2} \mathrm{WO}_{4}$} & \multicolumn{2}{|c|}{ W-DA2 } & \multicolumn{2}{|c|}{ W-DAn } \\
\hline & IR & Raman & IR & Raman & IR & Raman & IR & Raman \\
\hline \multirow{2}{*}{$\overline{\nu \nu(\mathrm{W}=\mathrm{O})}$} & 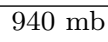 & $\overline{9940 \mathrm{vw}}$ & $950 \mathrm{~s}$ & $\overline{947 \mathrm{~s}}$ & $951 ? \mathrm{~m}$ & $900 \mathrm{~s}$ & & \\
\hline & & & & & $885 \mathrm{~s}$ & $888 \mathrm{~s}$ & $905 \mathrm{~s}$ & $890 \mathrm{~s}$ \\
\hline \multirow{8}{*}{$\nu(\mathrm{O}-\mathrm{W}-\mathrm{O})$} & & & & & $857 \mathrm{sb}$ & & $859 \mathrm{~s}$ & \\
\hline & & & & & $841 \mathrm{sb}$ & $846 \mathrm{~m}$ & & \\
\hline & $824 \mathrm{sb}$ & $809 \mathrm{~s}$ & & & $822 \mathrm{mb}$ & & $816 \mathrm{~m}$ & $813 \mathrm{~m}$ \\
\hline & $756 \mathrm{sb}$ & & & $774 \mathrm{mb}$ & & & $760^{1} \mathrm{sb}$ & \\
\hline & & $714 \mathrm{~m}$ & $740 \mathrm{sb}$ & & $724 \mathrm{mb}$ & $732 \mathrm{vw}$ & & \\
\hline & & & $680 \mathrm{sb}$ & & $674 \mathrm{sb}$ & $690 \mathrm{w}$ & $678 \mathrm{vw}$ & $679 \mathrm{w}$ \\
\hline & & $614 \mathrm{w}$ & & $638 \mathrm{sb}$ & $613 \mathrm{sb}$ & & & \\
\hline & & & & & & $594 \mathrm{vw}$ & $577 \mathrm{sb}$ & $576 \mathrm{vw}$ \\
\hline \multirow{4}{*}{ Organic } & & & & & & $550 \mathrm{vw}$ & $545 \mathrm{sb}$ & $552 \mathrm{vw}$ \\
\hline & & & & & $537 \mathrm{~s}$ & & & $519 \mathrm{w}$ \\
\hline & & & & & & & $487 \mathrm{w}$ & $490 \mathrm{vw}$ \\
\hline & & & & & & $460 \mathrm{w}$ & $474 \mathrm{w}$ & \\
\hline Inorganic & & $434 \mathrm{vw}$ & & $467 \mathrm{vw}$ & & $412 \mathrm{w}$ & & $436 \mathrm{vw}$ \\
\hline $\mathrm{W}-\mathrm{OH}_{2}$ & & & & $377 \mathrm{vw}$ & & & & $377 \mathrm{~m}$ \\
\hline \multirow{3}{*}{$\delta(\mathrm{O}-\mathrm{W}-\mathrm{O})$} & & $326 \mathrm{w}$ & & $330 \mathrm{vw}$ & & $348 \mathrm{w}$ & & $336 \mathrm{vw}$ \\
\hline & & & & & & $311 \mathrm{w}$ & & $301 \mathrm{vw}$ \\
\hline & & $274 \mathrm{~m}$ & & & & $261 \mathrm{~m}$ & & $283 \mathrm{vw}$ \\
\hline$\nu(\mathrm{W}-\mathrm{O}-\mathrm{W})$ & & $252 \mathrm{w}$ & & $236 \mathrm{w}$ & & $222 \mathrm{w}$ & & $226 \mathrm{w}$ \\
\hline \multirow{3}{*}{ Lattice modes } & & $187 \mathrm{w}$ & & $194 \mathrm{w}$ & & $201 \mathrm{~m}$ & & $182 \mathrm{vw}$ \\
\hline & & $136 \mathrm{w}$ & & $144 \mathrm{vw}$ & & & & $144 \mathrm{vw}$ \\
\hline & & $94 \mathrm{w}$ & & $106 \mathrm{w}$ & & & & \\
\hline
\end{tabular}

Table 3.5: Observed peaks in powder samples, $0-1000 \mathrm{~cm}^{-1} \cdot \nu$ stretching, $\delta$ deformation/in-plane bending, $\rho$ rocking; s strong, m medium, $\mathrm{w}$ weak, $\mathrm{vw}$ very weak, b broad.

${ }^{1} \rho\left(\mathrm{NH}_{3}^{+}\right)$mode. 
case in $\mathrm{WO}_{3} \cdot \mathrm{H}_{2} \mathrm{O}$ [66]. This may account for the multiple peaks observed in this region. There is a relationship between bond length (an indication of bond strength) and the frequency of the vibrational modes(s) for the terminal $\mathrm{W}=\mathrm{O}$ bond, namely that as the bond length decreases, bond strength and frequency increases $[60,66]$. It is not therefore unlikely to observe more than one terminal $\mathrm{W}=\mathrm{O}$ vibration, especially given the presence of superlattices and the fact that the unit cell is not just the simple $3.9 \AA \times 3.9 \AA$ expected for the simple square planar structure.

The remaining bands can be assigned as follows: $580-860 \mathrm{~cm}^{-1} \mathrm{O}-\mathrm{W}-\mathrm{O}$ stretching, 430-470 $\mathrm{cm}^{-1}$ inorganic Raman mode (W-O), 260-350 $\mathrm{cm}^{-1} \mathrm{O}-$ $\mathrm{W}-\mathrm{O}$ bending, 220-250 $\mathrm{cm}^{-1} \mathrm{~W}-\mathrm{O}-\mathrm{W}$ stretching, 90-200 $\mathrm{cm}^{-1}$ lattice modes [17]. These bands occur at similar positions in the hybrid compounds.

\section{The effect on the organic species}

Perhaps the most telling difference between W-DA2 and W-DAn $(n>2)$ is the presence or absence of the broad peak centred at $2100 \mathrm{~cm}^{-1}$ in the IR spectra. This feature is due to a combination of the asymmetrical $-\mathrm{NH}_{3}{ }^{+}$bending vibration and the torsional oscillation of the $-\mathrm{NH}_{3}{ }^{+}$group as it interacts with the apical oxygen of the tungsten oxide framework [245]. Both $-\mathrm{NH}_{2}$ scissoring and $-\mathrm{NH}_{3}{ }^{+}$bending occurs in the region $1580-1630 \mathrm{~cm}^{-1}$, however the latter displays three peaks (as seen in W-DAn) while the former yields only two (as in W-DA2). Furthermore, the N-H stretching bands occur at slightly higher energies in W-DAn compared with W-DA2 $\left(3240-3300 \mathrm{~cm}^{-1}\right.$, c.f. $3135-3250 \mathrm{~cm}^{-1}$ ) and these peaks are also much sharper. However, an increase in alkyl chain length should have little effect on the $\mathrm{N}-\mathrm{H}$ stretching frequency (as observed by Segal and Eggerton on mono- and diamines of different chain lengths [241]). The observed differences are discussed in the next section.

The presence of broad vibrational bands in the W-DAn spectra in the range $2430-2790 \mathrm{~cm}^{-1}$ can be ascribed to $\mathrm{NH}_{3}{ }^{+}$symmetrical stretching [245, 240] and often appear as a broad, ill-defined band under the $\mathrm{C}-\mathrm{H}$ stretching modes near $2800 \mathrm{~cm}^{-1}$ [18]. In $\mathrm{H}_{2} \mathrm{WO}_{4}$ a small peak is observed in the IR spectrum at $1614 \mathrm{~cm}^{-1}$, which corresponds to $\mathrm{H}_{2} \mathrm{O}$ bending. While the 
hybrid samples also exhibit peaks near this value $\left(1600-1630 \mathrm{~cm}^{-1}\right)$, these peaks are sharp and well defined, and the vibrational band at $\sim 3400 \mathrm{~cm}^{-1}$ (indicative of $\mathrm{O}-\mathrm{H}$ stretching) is absent. Thus we conclude that most of the co-ordinated water molecules $\left(\mathrm{W}-\mathrm{OH}_{2}\right)$ have been dehydrogenated during the organic intercalation process (a small feature at $377 \mathrm{~cm}^{-1}$ in the Raman spectra may correspond to $\mathrm{W}-\mathrm{O}-\mathrm{H})$. The peaks in the hybrid spectra in the range $1600-1630 \mathrm{~cm}^{-1}$ correspond instead to $\mathrm{NH}_{2}$ or $\mathrm{NH}_{3}{ }^{+}$bending.

As only the terminal amino/ammonium group is involved in interactions with the inorganic layer, it is no surprise that the bands corresponding to $\mathrm{C}-\mathrm{H}, \mathrm{C}-\mathrm{N}$ and $\mathrm{C}-\mathrm{C}$ vibrations remain virtually unchanged. The peaks in the hybrid spectra corresponding to these vibrations can be assigned as follows: 2850-2960 $\mathrm{cm}^{-1} \mathrm{C}-\mathrm{H}$ stretching, 1300-1500 $\mathrm{cm}^{-1} \mathrm{CH}_{2}$ wagging, bending, and scissoring, 950-1100 $\mathrm{cm}^{-1} \mathrm{C}-\mathrm{N}$ and C-C stretching. From $460-550 \mathrm{~cm}^{-1}$ there are a small number of unassigned organic bands (including a sharp peak in the IR at $537 \mathrm{~cm}^{-1}$ for W-DA2, corresponding to a bending vibration of the $\mathrm{N}-\mathrm{C}-\mathrm{C}-\mathrm{N}$ backbone $[235])$.

\section{The organic-inorganic bonding nature}

The most noticeable difference among the hybrid samples is that W-DA2 shows quite a different bonding nature from the hybrids with longer organic chains: there is no evidence for the presence of ammonium $\left(-\mathrm{NH}_{3}{ }^{+}\right)$terminal groups in W-DA2. As mentioned previously, W-DA2 lacks a feature at 2100 $\mathrm{cm}^{-1}$ found in the other hybrid spectra that corresponds to deformations of terminal $-\mathrm{NH}_{3}{ }^{+}$. Combined with the presence of the $-\mathrm{NH}_{3}{ }^{+}$rocking mode found at $770 \mathrm{~cm}^{-1}[240]$, this implies that some or all of the amine molecules in W-DAn appear as $\mathrm{R}-\mathrm{NH}_{3}{ }^{+} \ldots{ }^{-} \mathrm{O}-\mathrm{W}$, while all those of W-DA2 appear as neutral $-\mathrm{NH}_{2}$ species. This raises the question of how charge balance is achieved in W-DA2 and also whether hydrogen bonding is the exclusive mechanism of bonding, whereas in the longer organic molecule hybrids there is clearly an electrostatic component as well due to the presence of terminal ammonium groups. There are also differences in the $\mathrm{N}-\mathrm{H}$ stretch region, suggesting changes in the bonding chemistry as one progresses from DA2 to longer DAn alkyl chains. As mentioned earlier, the $\mathrm{N}-\mathrm{H}$ stretching bands 


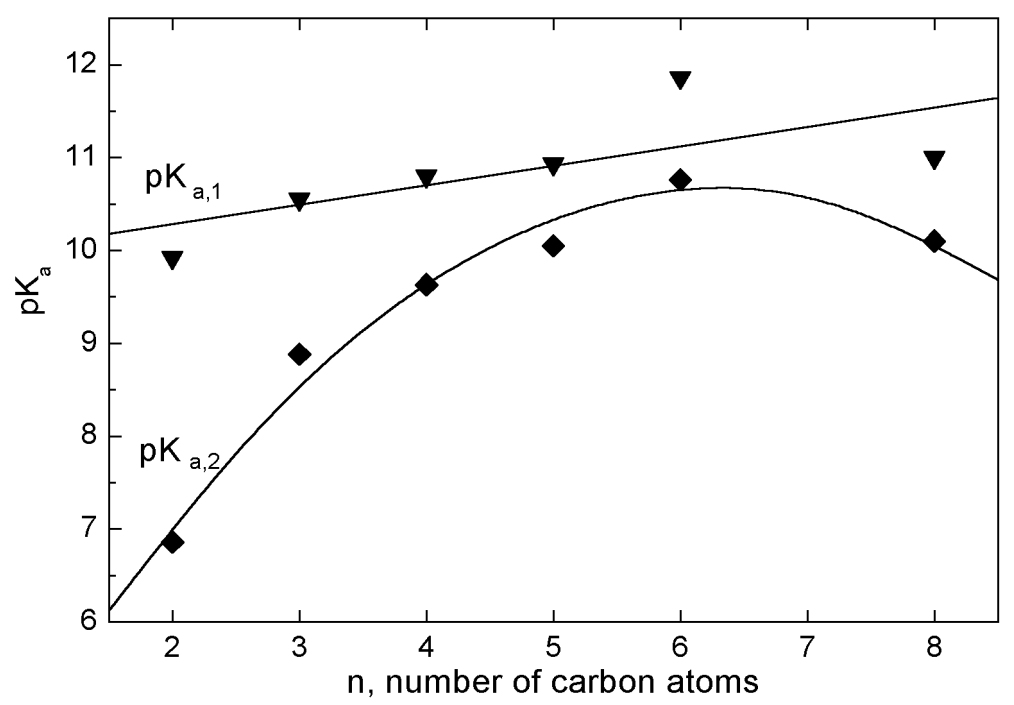

Figure 3.10: Plot of $\mathrm{pK}_{a}$ values from Ref. [174] versus the number of carbon atoms of $\alpha, \omega$-diamines in aqueous solution.

for W-DA2 appear at lower wavenumbers than for the longer-chain W-DAn species. Hydrogen bonding (which holds the DA2 molecules in place between the layers) will cause these bands to shift to lower frequencies [245]. Moreover, DA2 is the only case where the inductive effect on the terminal amine groups is known to be a perturbing factor to the molecular vibrations due to the shorter alkyl chain length [241].

One possible explanation for the observed difference in structure between DA2 and longer DAn molecules intercalated into an acidic metal oxide, arises from examining the trend in basicity of $\alpha, \omega$-diamines in solution, shown by the $\mathrm{pK}_{a}$ values in Figure 3.10. The $\mathrm{pK}_{a}$ of a compound is a measure of its strength as an acid, and in this case $\mathrm{pK}_{a, 1}$ and $\mathrm{pK}_{a, 2}$ are related to how much each of the following two reactions have proceeded:

$$
\begin{aligned}
& \mathrm{pK}_{a, 1}: \mathrm{H}_{3} \mathrm{~N}^{+}\left(\mathrm{CH}_{2}\right)_{n} \mathrm{NH}_{3}{ }^{+} \rightarrow \mathrm{H}_{2} \mathrm{~N}\left(\mathrm{CH}_{2}\right)_{n} \mathrm{NH}_{3}{ }^{+}+\mathrm{H}^{+} \\
& \mathrm{pK}_{a, 2}: \mathrm{H}_{2} \mathrm{~N}\left(\mathrm{CH}_{2}\right)_{n} \mathrm{NH}_{3}{ }^{+} \rightarrow \mathrm{H}_{2} \mathrm{~N}\left(\mathrm{CH}_{2}\right)_{n} \mathrm{NH}_{2}+\mathrm{H}^{+}
\end{aligned}
$$

Although the trend in basicity only changes slightly with increasing alkyl length for the monoamines (due to combinations of solution effect, steric hindrance to solvation, and number of available H-bonds $[8,10,30])$, a substantial increase in basicity is observed in going from DA2 to DA8. This increase 
in base strength for the diaminoalkanes is due to an increase of the inductive effect as the number of methylene groups $\left(-\mathrm{CH}_{2}-\right)$ increases. The formation of alkylammonium $\left(-\mathrm{NH}_{3}{ }^{+}\right)$end groups will therefore be more favourable for the more basic diaminoalkanes (i.e. diaminobutane and above). This apparent difference is also manifested in the TGA profile of these hybrids, with W-DA2 dissociating at a higher temperature than any of the other W-DAn compounds [48] (Section 3.2.4).

The strong Raman peak at $950 \mathrm{~cm}^{-1}$ in $\mathrm{H}_{2} \mathrm{WO}_{4}$ corresponds to an apical $\mathrm{W}=\mathrm{O}$ bond, which is characteristic of a layered structure. This peak is present in all of the hybrid compounds although it is shifted to lower wavenumbers of $890-900 \mathrm{~cm}^{-1}$, indicating that the apical oxygen is not as tightly bound to the tungsten as in $\mathrm{H}_{2} \mathrm{WO}_{4}$. This too can be expected as charge balance in $\mathrm{H}_{2} \mathrm{WO}_{4}$ is achieved by co-ordinated water molecules binding via the oxygen atom to the tungsten layer in an alternating updown checkerboard arrangement, leaving the remaining apical oxygen atoms to bind relatively strongly to the tungsten, with weaker hydrogen bonding holding the layers together. In the hybrid materials one might expect the influence of organic molecules on each apical oxygen to be more uniform, and stronger compared to the $\mathrm{W}-\mathrm{OH}_{2} \cdots \mathrm{O}=\mathrm{W}$ interaction, hence weakening the $\mathrm{W}=\mathrm{O}$ bond, and causing a lowering in frequency of the Raman shift.

\subsubsection{W-MAn samples}

Following the initial study on the inorganic parent materials $\mathrm{WO}_{3}$ and $\mathrm{H}_{2} \mathrm{WO}_{4}$, and the tungsten oxide diamine hybrids (W-DAn), further IR experiments were performed on tungsten oxide monoamine hybrids as they were synthesised. The IR spectra are given for a variety of monoamine hybrids in Figure 3.11 and tabulated in Table 3.6.

\section{The inorganic layer}

While the IR spectra of the W-DAn hybrids (Figure 3.8) display strong broad bands corresponding to stretching vibrations of the $\mathrm{O}-\mathrm{W}-\mathrm{O}$ framework, a very different signal is observed from the W-MAn hybrids. Instead 


\begin{tabular}{|c|c|c|c|c|}
\hline Assignment & W-MA2 & W-MA4 & W-MA6 & W-phen \\
\hline$\nu(\mathrm{O}-\mathrm{H})$ & $3475 \mathrm{wb}$ & $3502 \mathrm{wb}$ & $3387 \mathrm{wb}$ & $3413 \mathrm{wb}$ \\
\hline \multirow{3}{*}{$\nu\left(\mathrm{NH}_{2}, \mathrm{NH}_{3}{ }^{+}\right)$} & & 3197 vw & & \\
\hline & & $3131 \mathrm{w}$ & & $3057 \mathrm{w}$ \\
\hline & $3028 \mathrm{vw}$ & $3062 \mathrm{w}$ & $3030 \mathrm{w}$ & 3027 w \\
\hline \multirow{3}{*}{$\nu\left(\mathrm{CH}_{2}\right)$} & $2982 \mathrm{w}$ & $2962 \mathrm{~m}$ & $2958 \mathrm{~s}$ & $3004 \mathrm{vW}$ \\
\hline & $2932 \mathrm{w}$ & $2935 \mathrm{w}$ & $2928 \mathrm{~s}$ & $2932 \mathrm{vw}$ \\
\hline & & $2875 \mathrm{w}$ & $\begin{array}{l}2872 \mathrm{w} \\
2859 \mathrm{~m}\end{array}$ & $2899 \mathrm{vw}$ \\
\hline \multirow{4}{*}{$\begin{array}{l}\mathrm{NH}_{3}^{+} \\
\text {overtones }\end{array}$} & $2799 \mathrm{w}$ & $2762 \mathrm{w}$ & $2748 \mathrm{w}$ & $2749 \mathrm{w}$ \\
\hline & $2722 \mathrm{w}$ & $2653 \mathrm{w}$ & $2633 \mathrm{w}$ & $2652 \mathrm{w}$ \\
\hline & $2594 \mathrm{w}$ & $2522 \mathrm{w}$ & $2570 \mathrm{w}$ & $2564 \mathrm{w}$ \\
\hline & $2506 \mathrm{w}$ & $2410 \mathrm{w}$ & $2488 \mathrm{w}$ & $2471 \mathrm{w}$ \\
\hline$\nu\left(\mathrm{NH}_{3}{ }^{+} \cdots \mathrm{O}^{-}\right)$ & $2050 \mathrm{mb}$ & $1955 \mathrm{vwb}$ & $2012 \mathrm{wb}$ & $2010 \mathrm{wb}$ \\
\hline \multirow[t]{2}{*}{$\delta\left(\mathrm{NH}_{2} / \mathrm{NH}_{3}{ }^{+}\right)$} & $1655 \mathrm{w}$ & & & \\
\hline & $1606 \mathrm{~s}$ & $1598 \mathrm{~s}$ & $1605 \mathrm{~s}$ & $1605 \mathrm{~s}$ \\
\hline \multirow{3}{*}{$\begin{array}{l}\sigma\left(\mathrm{CH}_{2}\right), \\
\text { aromatic } \\
\nu(\mathrm{C}=\mathrm{C})\end{array}$} & $1504 \mathrm{~s}$ & $1497 \mathrm{~s}$ & $1498 \mathrm{~s}$ & $1497 \mathrm{~s}$ \\
\hline & $1471 \mathrm{~m}$ & $1471 \mathrm{w}$ & $1468 \mathrm{~m}$ & $1465 \mathrm{w}$ \\
\hline & $1458 \mathrm{w}$ & & & $1454 \mathrm{~m}$ \\
\hline \multirow{3}{*}{ sciss. $\mathrm{CH}_{2}$} & $1395 \mathrm{~s}$ & $1385 \mathrm{w}$ & $1384 \mathrm{w}$ & $1386 \mathrm{w}$ \\
\hline & 1346 w & & & $1325 \mathrm{w}$ \\
\hline & $1273 \mathrm{w}$ & & & $1262 \mathrm{w}$ \\
\hline$\omega\left(\mathrm{CH}_{2}\right)$ & $1219 \mathrm{~m}$ & & & $1147 \mathrm{~m}$ \\
\hline$\nu(\mathrm{C}-\mathrm{N})$ & $1198 \mathrm{~m}$ & $1165 \mathrm{w}$ & $1162 \mathrm{w}$ & $1112 \mathrm{w}$ \\
\hline$\nu(\mathrm{C}-\mathrm{C})$ & $1048 \mathrm{~m}$ & $1075 \mathrm{w}$ & $1093 \mathrm{w}$ & $1030 \mathrm{~m}$ \\
\hline$\nu(\mathrm{W}=\mathrm{O})$ & $944 \mathrm{w}$ & $963 \mathrm{w}$ & $933 \mathrm{~m}$ & $950 \mathrm{~m}$ \\
\hline \multirow{8}{*}{$\nu(\mathrm{O}-\mathrm{W}-\mathrm{O})$} & & & & $908 \mathrm{w}$ \\
\hline & $879 \mathrm{w}$ & $918 \mathrm{~s}$ & $867 \mathrm{w}$ & $877 \mathrm{~s}$ \\
\hline & $836 \mathrm{w}$ & $885 \mathrm{w}$ & $828 \mathrm{~m}$ & $837 \mathrm{~m}$ \\
\hline & $796 \mathrm{w}$ & $789 \mathrm{~s}$ & $794 \mathrm{w}$ & \\
\hline & $728 \mathrm{w}$ & $737 \mathrm{w}$ & $732 \mathrm{w}$ & $745 \mathrm{~m}$ \\
\hline & $680 \mathrm{~m}$ & & $690 \mathrm{~s}$ & 698 m \\
\hline & $654 \mathrm{w}$ & & & $659 \mathrm{~s}$ \\
\hline & $579 \mathrm{w}$ & & & $591 \mathrm{w}$ \\
\hline$\delta(\mathrm{C}-\mathrm{N})$ & $490 \mathrm{~m}$ & $530 \mathrm{~m}$ & $494 \mathrm{~m}$ & $486 \mathrm{~m}$ \\
\hline
\end{tabular}

Table 3.6: Observed IR peaks in W-MAn powder samples. $\nu$ stretching, $\delta$ deformation/in-plane bending, $\sigma$ bending, sciss. scissoring, $\omega$ wagging; $\mathrm{s}$ strong, m medium, w weak, vw very weak, b broad. 


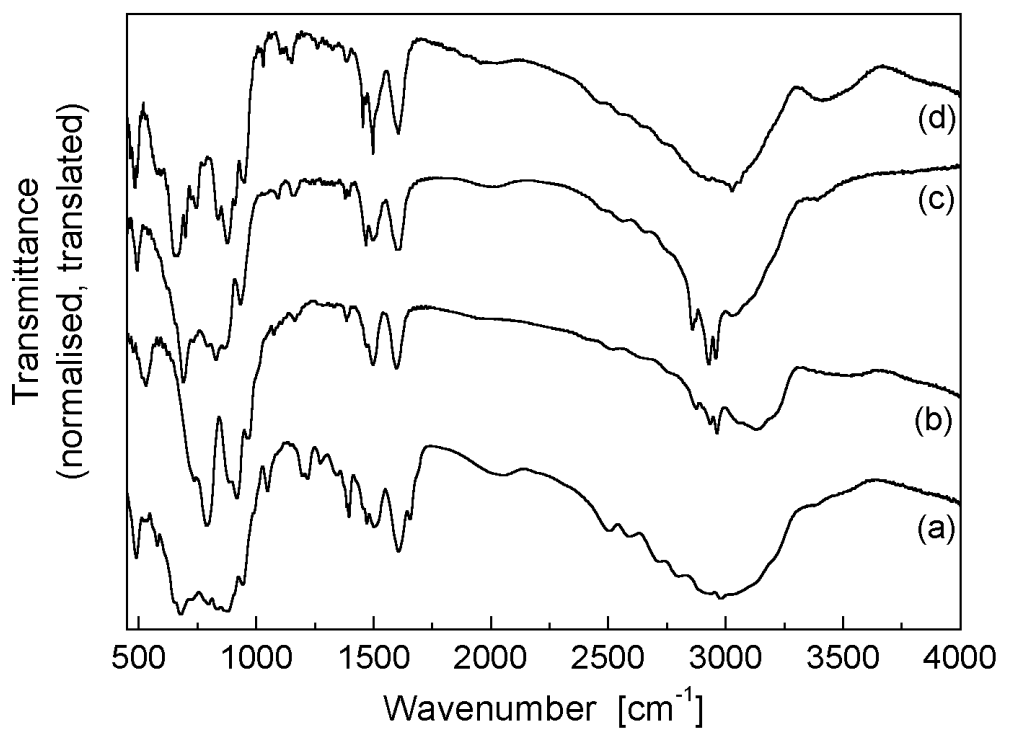

Figure 3.11: Infrared spectra of W-MAn hybrids: (a) W-MA2; (b) W-MA4; (c) W-MA6; (d) W-phen.

of a single broad band, two distinct features with additional splitting are observed. This is generally observed in well-ordered systems, indicating that the inorganic layer in the monoamine hybrids is more ordered than in the diamine hybrids [16]. One possible explanation is the greater degree of freedom for the stacking of the layers, as the organic molecules only tether to the inorganic layers at one end instead of two, which places extra restrictions on the inorganic structure. (One simple example is that organic diamines intercalated in the tungsten oxide or metal halide layered structure must have an even number of carbons in the backbone to ensure the conformations of the amine terminations presented to adjacent inorganic layers are symmetrical. However, metal halide-based hybrids have been synthesised with both even and odd numbered monoamine carbon chains [196].)

Once again, the apical $\mathrm{W}=\mathrm{O}$ bonds are identified with the stretching mode located at $\sim 950 \mathrm{~cm}^{-1}$ as for the other compounds, however it appears to be occluded somewhat by the presence of the expanded $\mathrm{O}-\mathrm{W}-\mathrm{O}$ framework features nearby. 


\section{The organic species}

In the W-DAn hybrids, the presence or absence of a broad band centred near $2100 \mathrm{~cm}^{-1}$ was an important feature, related to the presence of terminal $\mathrm{NH}_{3}{ }^{+}$ammonium groups. While all of the monoamine hybrids display some feature near this frequency, it is much less pronounced than for the diamine hybrids. Nevertheless, there are other features present in these spectra that are attributed to $-\mathrm{NH}_{3}{ }^{+}$: the broad 'hump' under the $\mathrm{C}-\mathrm{H}$ and $\mathrm{N}-\mathrm{H}$ stretching bands around $3000 \mathrm{~cm}^{-1}[18]$ and $-\mathrm{NH}_{3}{ }^{+}$overtones in the region from $2400-2800 \mathrm{~cm}^{-1}[240,245]$. The band near $1600 \mathrm{~cm}^{-1}$ is attributed to in-plane deformation of $-\mathrm{NH}_{3}{ }^{+}$, but also occurs at the same frequency for $-\mathrm{NH}_{2}$. The $-\mathrm{NH}_{2}$ bands at $3400 \mathrm{~cm}^{-1}$ and $1600 \mathrm{~cm}^{-1}$ are broadened due to hydrogen bonding.

Other prominent organic modes are assigned as follows: $2850-3000 \mathrm{~cm}^{-1}$ $\mathrm{C}-\mathrm{H}$ stretching (aliphatic $\mathrm{C}-\mathrm{H}$ occur at lower frequencies than aromatic $\mathrm{C}-$ $\mathrm{H}), 1300-1500 \mathrm{~cm}^{-1} \mathrm{CH}_{2}$ bending and scissoring, $1450-1500 \mathrm{~cm}^{-1}$ aromatic $\mathrm{C}=\mathrm{C}$ stretching in $\mathrm{W}$-phen, $1150-1200 \mathrm{~cm}^{-1} \mathrm{CH}_{2}$ wagging, $1100-1200$ $\mathrm{cm}^{-1} \mathrm{C}-\mathrm{N}$ stretching, $1030-1090 \mathrm{~cm}^{-1} \mathrm{C}-\mathrm{C}$ stretching, $490 \mathrm{~cm}^{-1} \mathrm{C}-\mathrm{N}$ bending.

\section{Organic-inorganic interaction}

As mentioned above, the broad feature at $2100 \mathrm{~cm}^{-1}$ which is normally fairly prominent in the diamine hybrids, is relatively weak in the monoamine hybrids. This band was identified in Section 3.3.1 (page 53) as arising from the bonding between the terminal $-\mathrm{NH}_{3}{ }^{+}$groups and the apical $\mathrm{W}=\mathrm{O}$ oxygen. It shifts to lower frequencies when the hydrogen bonding is weaker (as is seen in the monoamine hybrids) and decreases in intensity when symmetry is increased [61]. This suggests that, compared with the W-DAn hybrids, the interaction between the $-\mathrm{NH}_{3}{ }^{+}$terminal groups and the tungsten oxide layer is weaker but more symmetrical for the W-MAn hybrids.

Despite this, the organic molecules do not appear to interact with each other and hence are not closely-packed. Ordered arrangements of simple alkane-based organic molecules exhibit a series of sharp equidistant modes 
in the region $1150-1350 \mathrm{~cm}^{-1}$ [77], which are not observed in the W-MAn spectra. This finding is consistent with reported crystal structures of metal halide-monoamine hybrids, in which the methylene 'tails' of the organic molecules are quite disordered [200], reflecting the much weaker bonding there.

\subsubsection{Summary}

Infrared and Raman spectroscopy used together give a considerable amount of information regarding the inorganic structure of the hybrids, the state of the organic intercalates, and the bonding between them. The layered nature of the compounds is clearly seen from the presence of an intense line near $890 \mathrm{~cm}^{-1}$ in the Raman spectra and a feature near $950 \mathrm{~cm}^{-1}$ in the infrared spectra, corresponding to the apical $\mathrm{W}=\mathrm{O}$ bonds. The organic modes $(\mathrm{C}-\mathrm{H}$, $\mathrm{N}-\mathrm{H}, \mathrm{C}-\mathrm{C}, \mathrm{C}-\mathrm{N}$ ) are evident in the infrared spectra, and the presence of a broad infrared band located at $\sim 2100 \mathrm{~cm}^{-1}$ corresponds to the interaction between the terminal $-\mathrm{NH}_{3}{ }^{+}$groups of the organic and the apical oxygen atoms of the inorganic layer. This band is quite pronounced for the WDAn series (n geq 4), less pronounced for the W-MAn series, and completely absent (along with other $-\mathrm{NH}_{3}{ }^{+}$modes) in W-DA2. W-DA2 also behaved differently in the TGA experiment. The discrepancy in the behaviour of $\mathrm{W}$ DA2 relative to the other members of the W-DAn series may be related to changes in the basicity of the organic molecules as the carbon chain length increases. However, this does not address the issue of 'missing' charge which is required to balance the $\mathrm{WO}_{4}{ }^{2-}$ layers. It is speculated that in W-DA2 the protons may de-localise and serve to shield the apical oxygen atoms of the adjacent layers, although evidence has yet to been found to establish this.

\subsection{Structural and composition summary}

Tungsten oxide-based hybrid materials can be synthesised using various techniques, the best of which is via evaporation of solvent. The materials must be synthesised at a relatively high $\mathrm{pH}(\sim 10)$ to prevent the formation of Keggin ion clusters. 
Powder XRD patterns of the W-DAn hybrids exhibit a series of intense $00 \ell$ reflections, which correspond to the inter-inorganic layer separation. By examining the trend as a function of $n$, the number of carbon atoms in the organic molecule, it can be concluded that the inorganic layer consists of a single layer of corner-shared $\mathrm{WO}_{6}$ octahedra, with the organic molecules positioned perpendicular to it, and the terminal ammonium groups approximately in plane with the apical oxygen atoms. Elemental microanalysis also confirms the stoichiometry $\mathrm{WO}_{4} \cdot \mathrm{H}_{2} \mathrm{~N}\left(\mathrm{CH}_{2}\right)_{n} \mathrm{NH}_{2}$.

From the combined IR and Raman spectroscopy there is again evidence for a layered structure due to the presence of apical $\mathrm{W}=\mathrm{O}$ bands. The infrared spectra give us additional insight into the bonding nature of the organic molecule to the inorganic layer: in virtually all of the samples, there is a prominent band corresponding to the interaction between the $-\mathrm{NH}_{3}{ }^{+}$terminal groups and the apical oxygen atoms. The only material for which this is not the case is W-DA2, where not only is there no evidence for $-\mathrm{NH}_{3}{ }^{+}$, but also the clear presence of $-\mathrm{NH}_{2}$. This sample also has quite differing behaviour in the TGA analysis: while the other W-DAn samples show deintercalation of the organic molecules at $210-230^{\circ} \mathrm{C}, \mathrm{W}-\mathrm{DA} 2$ does not do so until $314^{\circ} \mathrm{C}$.

We have been unable to form single crystals of any of the hybrids large enough for single crystal x-ray diffraction (by far the best way to elucidate a crystal structure), most likely due to the harsh alkaline synthesis conditions required. Rietveld refinement of the powder XRD data has so far proved fruitless, as it is likely there is a larger supercell arising from distortion of the tungsten oxide layer (as seen in $\mathrm{H}_{2} \mathrm{WO}_{4}$, Figure 1.5). The presence of such a supercell is confirmed from selected area electron diffraction patterns. 


\section{Chapter 4}

\section{Ab Initio Computations}

Ab initio computation is an increasingly available tool for researchers, with the increasing performance and availability of efficient computing resources. As a modelling technique it can be used to estimate properties and predict behaviours of systems, which may otherwise be difficult or impossible to do experimentally.

In this case, we wished to explore the electronic properties of the tungsten oxide-based hybrid materials, based on the layered structure obtained from the experimental results. In preparation, and to test the suitability of the code used, an initial study of the alkali tungsten bronze series $\left(\mathrm{M}_{x} \mathrm{WO}_{3}, \mathrm{M}=\right.$ $\mathrm{H}, \mathrm{Li}, \mathrm{Na}, \mathrm{K}, \mathrm{Rb}$, and $\mathrm{Cs}$ ) was undertaken and compared with experimental and computational literature results.

From here a more detailed study of the variable doping of $\mathrm{Na}_{x} \mathrm{WO}_{3}(0 \leq$ $x \leq 1$ ) and oxygen-deficient $\mathrm{WO}_{3-x}$ was undertaken. The trends in either of these series could potentially be seen in the layered tungsten oxide hybrid systems upon doping.

Finally, the same computational techniques were applied to some simple layered organic-inorganic tungsten oxide hybrid structures to predict their electronic properties. The computational results are then compared with experiment by considering the optical spectra of $\mathrm{WO}_{3}$ and the W-DAn hybrids.

First the computational technique used will be discussed, before giving a detailed summary of the major results. 


\subsection{Introduction to technique}

The rapid improvement of computer performance over the last decade has enabled the development of software for performing quantum mechanical calculations of polyatomic systems. The number of atoms in the system is restricted only by the computer performance and memory available and at the time of writing, is typically of the order of 50 atoms. While there are many such programs available, this section will concentrate on the Vienna Ab-initio Simulation Package (VASP), the particular code utilised for this research. VASP uses a local-density approximation to the density functional theory with ultra-soft plane wave pseudopotentials [146, 147, 148, 149].

A brief summary of these terms is as follows:

\section{Density functional theory}

Density functional theory (DFT) was developed by Hohenberg and Kohn [116] and Kohn and Sham [140]. In short, the total energy of a system (including exchange and correlation energies) can be written as a functional of the electron density of that system, even in the presence of some static ionic potential. The minimum energy of that functional is the ground-state energy of the system and the corresponding electron density is the groundstate density. It is then also possible to convert the many-electron problem to an exactly equivalent set of self-consistent one-electron equations with an effective potential, thus simplifying the mathematics considerably.

\section{Local-density approximation}

The local-density approximation (LDA) to the DFT was also developed by Kohn and Sham [140]. By assuming that the exchange-correlation energy of the electrons is purely local, one can express this energy at a single point in the electron gas as if this gas was homogeneous with the same electron density as the original. Thus the LDA ignores any inhomogeneities in the electron density at each point, yet despite this, the LDA is often surprisingly effective. The LDA gives a single global minimum for the energy of electrons 
in a fixed ionic potential. However for spin-polarised systems where there may be multiple local minima, the approximation then fails. LDA is notorious for underestimating band gaps, so care must be taken when interpreting the resulting band structures.

\section{Generalised gradient approximation}

The generalised gradient approximation (GGA) is one further step up from the LDA [220]. It includes the second-order (linear) terms in the expression of the electron gas. The GGA accounts for the density inhomogeneity that LDA ignores. The total energy calculated using the GGA is often more accurate than that obtained using the LDA alone. In the limit where the gradient term becomes zero, the GGA reverts to the LDA, as expected.

\section{Ultra-soft plane-wave pseudopotentials}

Bloch's theorem (see Section 1.1.1) states that in a periodic solid each electronic wavefunction can be written as a product of a cell-periodic part and a wavelike part. Both of these can be expressed as a sum of plane waves, resulting in the electronic wavefunction

$$
\phi_{i}(r)=\sum_{G} c_{i, \mathbf{k}+\mathbf{G}} \exp [i(\mathbf{k}+\mathbf{G}) \cdot \mathbf{r}]
$$

This expression then describes an infinite, but discrete set of plane-waves. However the set can be truncated to consider only those plane-waves with kinetic energy less than some cut-off value, as these are the most important to consider. While this introduces a discrepancy in the total calculated energy of the system, this can be controlled by increasing the cut-off energy and repeating a calculation until the energy has suitably converged.

Only the valence electrons are individually calculated; the effects of the core electrons and nucleus are combined and expressed as a single pseudopotential function. This reduces the computation time dramatically and at little cost to the accuracy, as the core electrons rarely influence the crystal structure of a material. Ultra-soft pseudopotentials utilise this by describing the electronic potential for the two regions $r<r_{\text {core }}$ and $r>r_{\text {core }}$. For 
$r<r_{\text {core }}$ the pseudo-wavefunctions are allowed to be as soft as possible (by removing the norm-conserving constraint and making the wavefunction as smooth as possible) and hence do not require a large cut-off energy to accurately describe the system. The wavefunctions in the two regions are married together to be as smooth as possible, and for $r>r_{\text {core }}$ it matches the all-electron model exactly (as the valence electrons are the only ones in consideration in this regime).

Each approximation made introduces a small amount of uncertainty to the final result. DFT with the LDA is often able to predict lattice constants to within $1 \%$.

The input parameters VASP requires are as follows:

- Atomic type and positions within the unit cell (up to 50 atoms),

- $k$-space mesh to use (larger $k$-meshes result in longer computation times and require more computer memory, but give more accurate results),

- Pseudopotentials for each atomic species (see Table 4.1),

- Experimental parameters: energy cut-off values, etc. ([302], Ch. 7).

Examples of each of these are given in Appendix B.

The output files from a VASP run include:

- Unit cell and atomic positions for each species,

- Charge density map,

- Wavefunction map,

- Density of states (if selected, can also break down into individual atomic components),

- Band structure can be obtained indirectly,

- Ground state energy of the system.

More information can be found in the VASP guide [302] and references therein. 


\begin{tabular}{cc}
\hline Atom & Valence Electrons \\
\hline \hline $\mathrm{W}$ & $6 s^{1} 5 d^{5}$ \\
$\mathrm{O}$ & $2 s^{2} 2 p^{4}$ \\
$\mathrm{H}$ & $1 s^{1}$ \\
$\mathrm{Li}$ & $2 s^{1}$ \\
$\mathrm{Na}$ & $3 s^{1}$ \\
$\mathrm{~K}$ & $4 s^{1}$ \\
$\mathrm{Rb}$ & $5 s^{1}$ \\
$\mathrm{Cs}$ & $6 s^{1}$ \\
$\mathrm{C}$ & $2 s^{2} 2 p^{2}$ \\
$\mathrm{~N}$ & $2 s^{2} 2 p^{3}$ \\
\hline
\end{tabular}

Table 4.1: Valence states of the elements used in the calculations.

\subsubsection{Computational details}

We have used VASP with the generalised gradient approximation (GGA). The pseudopotentials in these calculations are ultrasoft Vanderbilt type pseudopotentials [267] as supplied by G. Kresse and J. Hafner [150]. The pseudopotential valence states for all elements used in this work are given in Table 4.1, with a plane-wave cut-off energy of $396 \mathrm{eV}$.

$k$-point meshes between $4 \times 4 \times 4$ and $15 \times 15 \times 15$ were used to relax the various systems, corresponding respectively to $24-36$ and $120-455 k$-points in the irreducible Brillouin zone. As a general rule, the simplest bronze structures with few (1-4) formula units per cell used finer $k$-point meshes (more $k$-points) than the larger non-metallic $\mathrm{WO}_{3-x}$ (small $x$ ) and hybrid structures. In all cases the $k$-point mesh was varied at the end of the relaxation and the energy was found to converge to better than $10 \mathrm{meV}$ in all cases. The cell parameters and atomic positions were allowed to relax in alternate cycles. The atomic positions were considered relaxed when the total energy had converged to within $10 \mathrm{meV}$ between ionic steps.

To study the effect of fractional doping in the $\mathrm{Na}_{x} \mathrm{WO}_{3}$ system, we used a supercell method consisting of up to eight primitive $\mathrm{WO}_{3}$ cells, with between 1-7 of these cells occupied by sodium ions in a pseudo-random fashion. Similarly for the oxygen-deficient $\mathrm{WO}_{3-x}$ system, 3-6 cells were used with one oxygen vacancy in each case. In all cases the basis vectors of the cell were 
chosen to avoid the creation of lines or planes of dopants. In the calculations of the hybrid structures the relative positions of the in-plane tungsten and oxygen atoms were fixed at the origin and half-way along each planar axis, respectively.

Finally we note that with the exception of the oxygen molecule total energy, none of the calculations here were spin-polarised. The total energy of the oxygen molecule in its ${ }^{3} \epsilon_{g}^{-}$ground state, used to compute energies of formation for the $\mathrm{WO}_{3-x}$ series, was computed in a $16 \AA$ cubic cell with convergence of the energy to within $10 \mathrm{meV}$. Our binding energy for the oxygen molecule of $3.214 \mathrm{eV} /$ atom is consistent with other calculated values reported in the literature [213].

\subsection{Previous studies}

There have been several studies by different groups using different computational packages on the crystal and electronic structure of tungsten oxide and the tungsten bronzes. The complexity of the systems has increased as computational resources have become more elaborate.

One early report is that of Bullett in 1983 [35], where the bulk and surface electronic states of $\mathrm{WO}_{3}$ and $\mathrm{NaWO}_{3}$ were studied using a non-relativistic atomic orbital-based method. Density of states, band structure were reported and compared with experimental results using UPS and XPS.

Hjelm et al. reported the electronic structure of $\mathrm{WO}_{3}, \mathrm{LiWO}_{3}, \mathrm{NaWO}_{3}$ and $\mathrm{HWO}_{3}$ in 1996 and modelled the UV-visible spectra from the density of states calculations [114]. They used a full-potential linear muffin-tin orbital method, which includes all of the electrons in the system instead of just the valence electrons plus the core electrons and nucleus as a single unit. They report density of states for all four systems, charge density for $\mathrm{WO}_{3}$ and $\mathrm{LiWO}_{3}$, a comparison of the density of states of cubic and hexagonal $\mathrm{WO}_{3}$, and an energy study of $\mathrm{HWO}_{3}$ where the hydrogen atom is shifted relative to the centre of the cell. Lastly the optical properties were calculated for $\mathrm{Li}_{x} \mathrm{WO}_{3}$ and $\mathrm{Na}_{x} \mathrm{WO}_{3}, x=0, \frac{1}{4}, \frac{1}{2}, \frac{3}{4}, 1$.

In 1997 Corà et al. used a full-potential linear muffin-tin orbital method 
with the LDA to study the electronic structures of $\mathrm{WO}_{3}, \mathrm{ReO}_{3}$ and $\mathrm{NaWO}_{3}$ [58]. They investigated the effect of adding sodium to $\mathrm{WO}_{3}$ compared with changing the valence state of the tungsten (i.e. replacing $\mathrm{W}^{6+}$ with $\mathrm{Re}^{5+}$ ). They report band structures and density of states of all three compounds.

Corà and Catlow also published another article in 2000 studying the different structures of tungsten and molybdenum oxides [57]. They used $a b$ initio Hartree-Fock calculations to study the stability of the different structural phases of $\mathrm{WO}_{3}$ and $\mathrm{MoO}_{3}$, namely perovskite, Mo-L layers, hexagonal, and pyrochlore. They discuss how other polymorphs might be constructed.

Late in 2004, after the majority of our calculations were complete, a paper was published in Phys. Rev. B by Walkingshaw et al. [271]. They used the SIESTA package, which is technically quite similar to VASP in that it uses DFT with the LDA. Like Corà et al. [58] they studied the effect of adding dopant electrons to cubic $\mathrm{WO}_{3}$ without adding any intercalated atom, for $x$

in $\frac{1}{16}$ intervals from zero to one. At each step they calculate the minimum energy structure and use this to construct phase diagrams. Because the calculations were performed on supercells of $\mathrm{WO}_{3}$ instead of single cells (as was the case in the other publications), the correct 'pseudocubic' phases of $\mathrm{WO}_{3}$ were identified.

\subsection{Cubic and hexagonal alkali bronzes}

\subsubsection{Structure}

The alkali elements ( $\mathrm{H}, \mathrm{Li}, \mathrm{Na}, \mathrm{K}, \mathrm{Rb}$ and $\mathrm{Cs})$ were each used as the intercalated species in cubic $\mathrm{MWO}_{3}(x=1$ bronzes $)$ and hexagonal $\mathrm{M}_{0.33} \mathrm{WO}_{3}$ (full intercalation of the hexagonal tunnels). As mentioned in Section 1.3, $\mathrm{WO}_{3}$ does not form a perfectly cubic cell at room temperature, as the $\mathrm{WO}_{6}$ octahedra are slightly distorted in terms of $\mathrm{W}-\mathrm{O}$ bond lengths and $\mathrm{W}-\mathrm{O}-\mathrm{W}$ bond angles, due to antiferroelectric displacement of the tungsten atoms and subsequent rotation of the $\mathrm{WO}_{6}$ octahedra $[35,82]$. However this is not taken into account in the calculated system; the cell always relaxes to a cubic structure. In calculations involving supercells of tungsten trioxide the distortions 


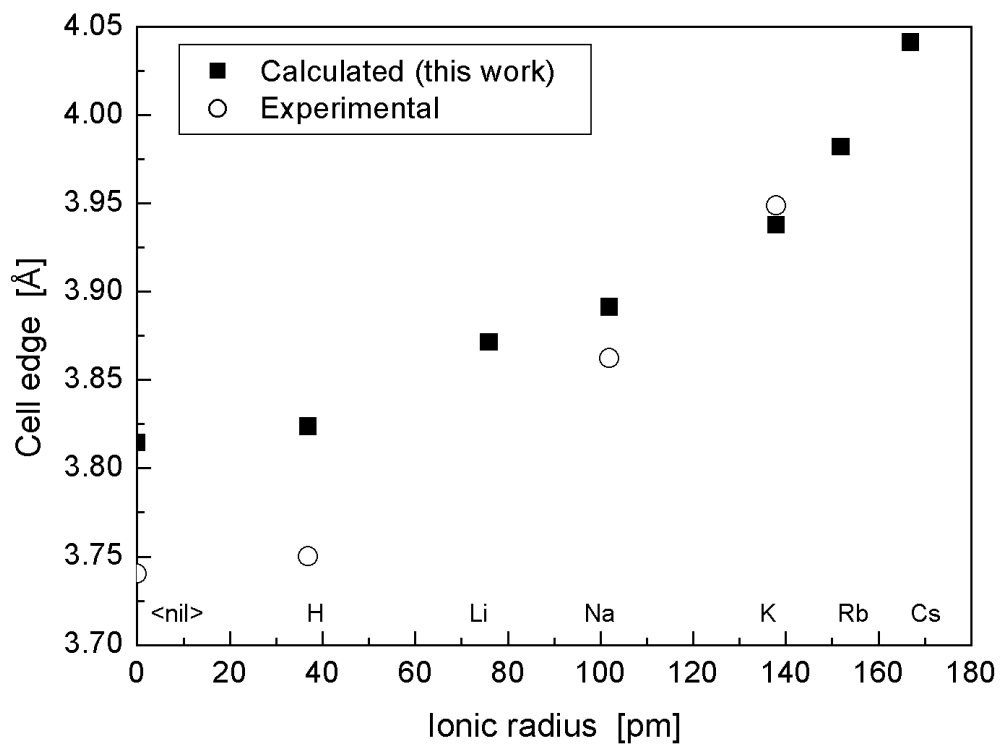

Figure 4.1: Calculated and experimental values of the cell parameter for fully intercalated cubic tungsten bronzes. Experimental values are obtained from $[33,38,94,234]$ and extrapolated to $x=1$.

are seen [271]. Tungsten bronzes on the other hand do form the simple cubic structure at high intercalation levels [33]. The hexagonal structure, which was also studied for comparison, is the same for both the oxide and bronzes. (The term 'cubic' is used connotatively throughout this article of those systems that are cubic or close to it; as opposed to the hexagonal systems also studied.)

Figure 4.1 shows some experimental results for the cubic bronze system (obtained from $[33,38,94,234]$, extrapolated to $x=1$. In the case of non-cubic $\mathrm{WO}_{3}$ a cubic cell was calculated from the volume average of the given parameters.) Of all the intercalated alkali elements which have been attempted experimentally, only sodium is able to form a stable structure with $x=1$ at normal temperatures and pressures. In general the calculated cell parameters for the cubic system are larger than the experimental. It is also noticeable that as the size of the intercalated ion increases, the cell size increases super-linearly. The Goldschmidt tolerance factor for cubic perovskites can be calculated from the formula $t=\frac{r_{M}+r_{O}}{\sqrt{2}\left(r_{W}+R_{O}\right)}[97]$, where $r_{j}$ are the ionic radii. For a perovskite structure to be stable, $t$ must be less than 


\begin{tabular}{ccccc}
\hline Compound & Expt. $a(\AA)$ & Calc. $a(\AA)$ & Expt. $c(\AA)$ & Calc. $c(\AA)$ \\
\hline \hline $\mathrm{WO}_{3}$ & 7.298 & 7.4103 & 3.899 & 3.8144 \\
$\mathrm{H}_{0.33} \mathrm{WO}_{3}$ & 7.38 & 7.4173 & 3.78 & 3.8111 \\
$\mathrm{Li}_{0.33} \mathrm{WO}_{3}$ & 7.405 & 7.4007 & 3.777 & 3.8219 \\
$\mathrm{Na}_{0.33} \mathrm{WO}_{3}$ & 7.38 & 7.4034 & 3.775 & 3.8248 \\
$\mathrm{~K}_{0.33} \mathrm{WO}_{3}$ & 7.37 & 7.4010 & 3.77 & 3.8282 \\
$\mathrm{Rb}_{0.33} \mathrm{WO}_{3}$ & 7.38 & 7.4163 & 3.78 & 3.8289 \\
$\mathrm{Cs}_{0.33} \mathrm{WO}_{3}$ & 7.38 & 7.4507 & 3.785 & 3.8342 \\
\hline
\end{tabular}

Table 4.2: Experimental cell parameters for hexagonal tungsten oxide and bronzes (from $[82,14]$ ), compared with our calculated results for hexagonal tungsten bronzes with hexagonal sites completely occupied.

unity. The tolerance factors for $\mathrm{NaWO}_{3}$ and $\mathrm{KWO}_{3}$ are 0.909 and 1.056 respectively, indicating that the potassium atom is slightly too large to form a stable structure. Potassium bronzes have been formed with high $x$ contents, but only under high-pressure synthesis conditions [38]. Rubidium and caesium cubic bronzes with high $x$ content cannot be formed. The hexagonal structure, having larger tunnels, is able to accommodate larger ions than observed in the cubic system. In the experimental system the stability of hydrogen is notoriously difficult to maintain, as it is quite mobile due to its small size. Both hydrogen and lithium are small enough ions to be able to occupy sites within the small triangular tunnels between the hexagonal tunnels [82]. Thus reported experimental results for $\mathrm{H}^{+}$- and $\mathrm{Li}^{+}$-hexagonal bronzes may show differing behaviour from larger atoms because the occupied sites may be different in each case. In the experimental hexagonal system, the $a$ parameter is observed to increase as ions are intercalated while the $c$ parameter decreases. The final values are relatively consistent across the series, as given in Table 4.2. These are generally lower than the calculated values we have obtained, although most discrepancies are less than $0.5 \%$.

For the hexagonal system, the changes in the lattice parameters are much less pronounced than in the cubic system, as shown in Table 4.3. This is due to the hexagonal tunnel spaces being much larger than the interstitial vacancies in the cubic system and so the interactions between the inserted ion and the $\mathrm{WO}_{3}$ lattice are smaller. 


\begin{tabular}{ccc}
\hline Compound & Cubic $\left(\AA^{3}\right)$ & Hexagonal $\left(\AA^{3}\right)$ \\
\hline \hline $\mathrm{WO}_{3}$ & 55.4977 & 60.4648 \\
$\mathrm{H}_{x} \mathrm{WO}_{3} 1$ & 55.8946 & 60.5278 \\
$\mathrm{Li}_{x} \mathrm{WO}_{3}$ & 58.0127 & 60.4270 \\
$\mathrm{Na}_{x} \mathrm{WO}_{3}$ & 58.9116 & 60.5158 \\
$\mathrm{~K}_{x} \mathrm{WO}_{3}$ & 61.0457 & 60.6785 \\
$\mathrm{Rb}_{x} \mathrm{WO}_{3}$ & 63.1294 & 60.7928 \\
$\mathrm{Cs}_{x} \mathrm{WO}_{3}$ & 65.9809 & 61.4445 \\
\hline
\end{tabular}

Table 4.3: Calculated cell volume per $\mathrm{WO}_{3}$ unit for cubic and hexagonal tungsten oxide and bronzes.

${ }^{1} x=1$ for cubic, $x=0.33$ for hexagonal.

\subsubsection{Charge-density}

Charge-density plots of the cubic system taken in a plane through the centre of the cell where the intercalated atom sits, reveal that hydrogen behaves differently from the other intercalates. This is shown in Figure 4.2. Bearing in mind that the charge-density plots consider only the valence electrons, the fact that we observe some charge on the hydrogen atom but not on any of the others indicates that hydrogen is not ionised. That larger atoms are completely ionised is consistent with other reported experimental results [35]. It is interesting that the same phenomenon occurs in both the cubic and hexagonal cases. The first ionisation energy of the intercalates are as follows: hydrogen $1.318 \mathrm{eV}$, lithium $0.526 \mathrm{eV}$, sodium $0.502 \mathrm{eV}$, potassium $0.425 \mathrm{eV}$, rubidium $0.409 \mathrm{eV}$, caesium $0.382 \mathrm{eV}[12]$. The high ionisation energy of hydrogen with respect to the other intercalates may be responsible for the differing behaviour.

\subsubsection{Energies of formation}

The energies of formation of the cubic and hexagonal tungsten bronzes are shown in Table 4.4 and Figure 4.3. These are calculated by subtracting the ground-state energies of the components $\left(\mathrm{WO}_{3}\right.$ plus the metal cation) from the ground-state energy of the final product (tungsten bronze). A negative energy of formation therefore indicates that the compound formed is stable. 


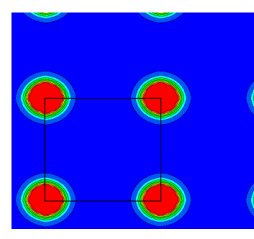

$\mathrm{WO}_{3}$

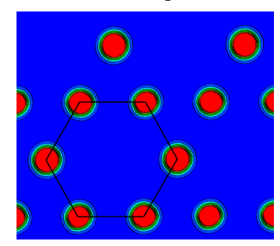

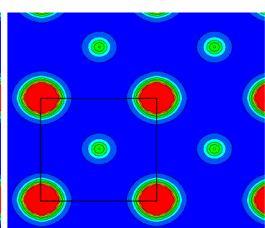

$\mathrm{HWO}_{3}$

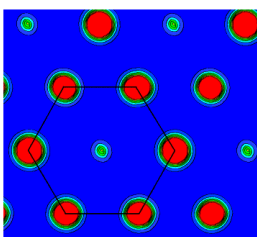

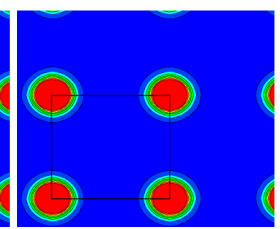

$\mathrm{NaWO}_{3}$

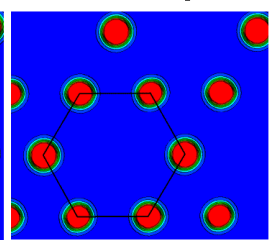

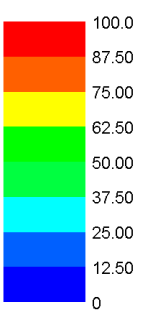

Figure 4.2: Charge density maps of cubic (top) and hexagonal (bottom) tungsten oxide and bronzes. The largest tunnels are indicated, which are completely filled in each case. The oxygen atoms in-plane with the intercalated atom can be clearly seen, and only in the case of hydrogen is the charge still associated with the intercalated atom.

\begin{tabular}{ccc}
\hline Compound & Cubic $(\mathrm{eV})$ & Hexagonal $(\mathrm{eV})$ \\
\hline \hline $\mathrm{WO}_{3}$ & 0 & -0.016 \\
$\mathrm{H}_{x} \mathrm{WO}_{3}^{1}$ & 3.059 & 1.047 \\
$\mathrm{Li}_{x} \mathrm{WO}_{3}$ & -2.023 & -0.560 \\
$\mathrm{Na}_{x} \mathrm{WO}_{3}$ & -2.373 & -0.813 \\
$\mathrm{~K}_{x} \mathrm{WO}_{3}$ & -2.354 & -1.132 \\
$\mathrm{Rb}_{x} \mathrm{WO}_{3}$ & -2.039 & -1.203 \\
$\mathrm{Cs}_{x} \mathrm{WO}_{3}$ & -0.637 & -1.245 \\
\hline
\end{tabular}

Table 4.4: Calculated energies of formation per $\mathrm{WO}_{3}$ unit for cubic and hexagonal tungsten oxide and bronzes, relative to cubic $\mathrm{WO}_{3}$.

${ }^{1} x=1$ for cubic, $x=0.33$ for hexagonal. 


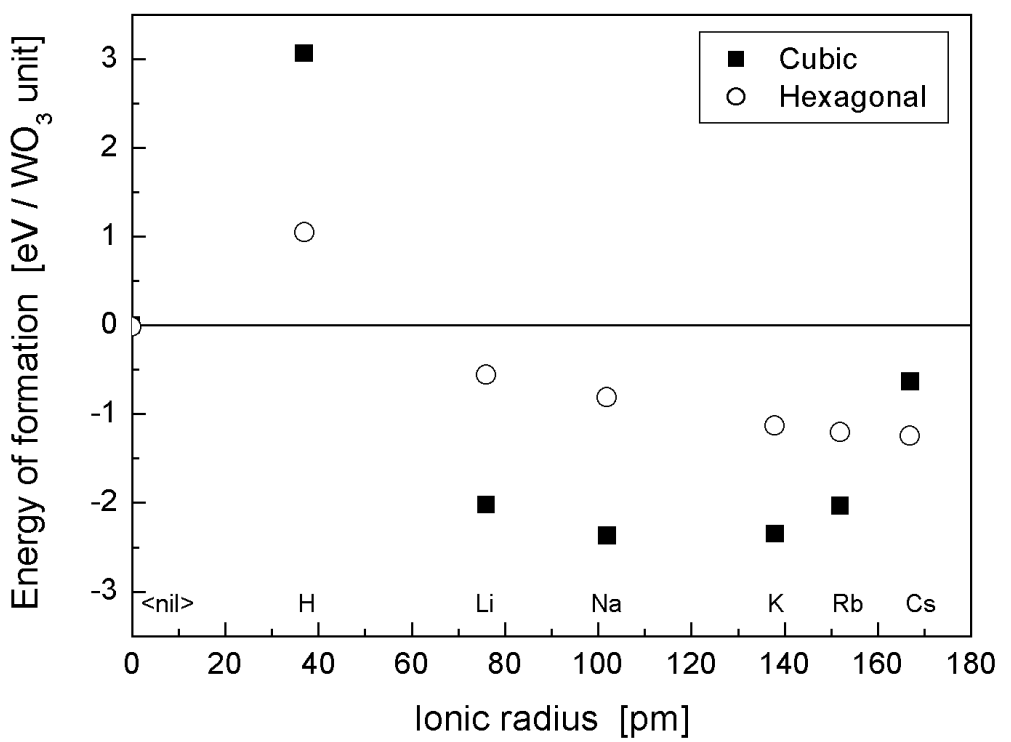

Figure 4.3: Calculated energies of formation per $\mathrm{WO}_{3}$ unit for cubic and hexagonal tungsten bronzes, relative to cubic $\mathrm{WO}_{3}$.

Firstly, a comparison of cubic and hexagonal $\mathrm{WO}_{3}$ shows that the hexagonal phase has a very similar energy to the cubic phase. Literature results indicate that the cubic phase is preferred, although the hexagonal phase is stable up to temperatures of $400-500^{\circ} \mathrm{C}$, indicating that the phase transition has a high activation energy $[82,207], \gg 50 \mathrm{meV}$. In both the cubic and hexagonal systems, the hydrogen-intercalated bronze energy is positive and large, indicating that the hydrogen bronzes are not stable. In the experimental system the hydrogen bronzes are easily oxidised as the protons are highly mobile $[94,115]$. This result also relates to hydrogen being the only intercalate that does not ionise in the bronze structures, as evidenced by the charge density plots earlier. For the hexagonal bronzes (apart from hydrogen) there is a steady trend in the energy of formation to more negative values as the size of the intercalated alkali metal ion increases. Therefore the larger ions form more stable bronzes than the smaller ones, which is also indicated in the literature [14]. In the cubic system however, the energy drops to a minimum and then, beyond $\mathrm{Na}$, becomes less negative in the case of the larger intercalates. This point coincides with the maximum ion size predicted by the Goldschmidt perovskite tolerance factor. For the experimentally stable 
compounds $\left(\mathrm{WO}_{3}, \mathrm{LiWO}_{3}\right.$, and $\left.\mathrm{NaWO}_{3}\right)$ there is a progression of the energy of formation to more negative values. This supports experimental evidence that sodium may well be the most stable of the cubic bronzes, as it is the only one for which a fully intercalated compound has been reported [115]. More work is required on the potassium, rubidium and caesium bronzes, as while these exhibit a negative energy of formation and are predicted from these calculations to be stable, they cannot be formed experimentally.

\subsubsection{Density of states}

For both the cubic and hexagonal systems other than hydrogen, the basic band structures of the bronzes are essentially identical to the parent oxide of the same phase. The only difference amongst them is the position of the Fermi level relative to the valence and conduction bands, which will be discussed later. Hence comparing the band structure of hexagonal and cubic $\mathrm{WO}_{3}$ will aid a great deal in describing the bronze systems. A comparison of the density of states for cubic and hexagonal $\mathrm{WO}_{3}$ is shown in Figure 4.4. The lowest band, situated at -18 to $-16 \mathrm{eV}$, corresponds to the oxygen $2 \mathrm{~s}$ orbitals. This band is present in all of the tungsten-oxide-based systems studied to date and always occurs at the same energy regardless of the structure or the presence of intercalated atoms or molecules.

The broad valence band, from -7 to $0 \mathrm{eV}$, is comprised mainly of oxygen $2 p$ orbitals. There is a small tungsten $5 d$ component but this is negligible above $-2 \mathrm{eV}$. The valence band cut-off is sharp at $0 \mathrm{eV}$ and coincides in both the cubic and hexagonal case with the Fermi level, rendering the oxide materials semiconducting. The conduction band lies from 0.5 to $5 \mathrm{eV}$ in both the cubic and hexagonal cases. In the cubic case it is solely comprised of tungsten $5 d$ orbitals; however in the hexagonal case there is also some additional oxygen $2 p$ contribution - particularly to the strong peak feature observed at $2.5 \mathrm{eV}$. The band gap (defined as the difference in energy between the top of the valence band and the bottom of the conduction band) is 0.4 $\mathrm{eV}$ in the cubic system and $0.5 \mathrm{eV}$ in the hexagonal. This is much less than the observed band gap, which is typically reported in the range of $2.5-3$ 


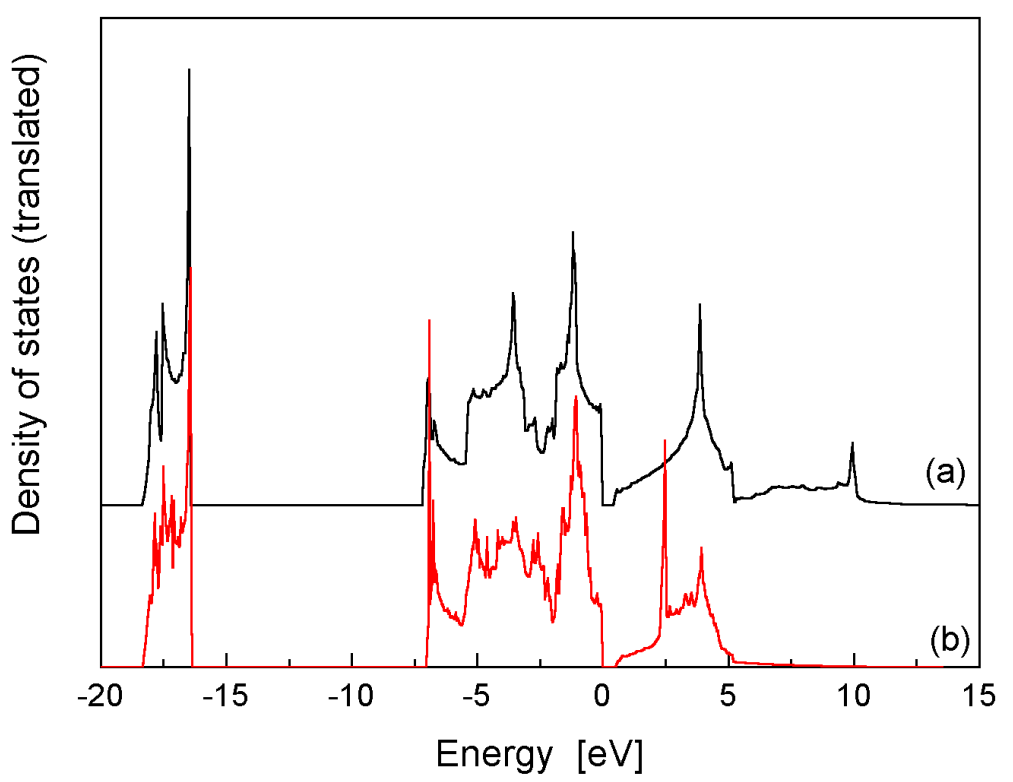

Figure 4.4: The density of states as calculated for cubic (a) and hexagonal (b) $\mathrm{WO}_{3}$.

$\mathrm{eV}$, and as being indirect $[58,100,172]$. However this is not too surprising as DFT generally underestimates band gaps. The presence of peak features in the density of states can also lead to the phenomenon where even though the conduction band is being filled, there is a sudden increase in the population of the conduction band at these peaks and a sharp transition in the optical spectrum is observed. Further, the density of states above the band gap is rather weak and would only contribute weakly to optical transitions. This is discussed in Section 4.7.

It is worth taking pause here to point out the similarities between this work and that of experimental results and other calculations reported on the same structures. Similar to the present findings, X-ray photoelectron spectroscopy (XPS) reveals that the valence band is comprised of oxygen $2 p$ states only, and the conduction band of tungsten $5 d$ states $[19,215]$. The oxygen $2 \mathrm{~s}$ state at $\sim-20 \mathrm{eV}$ has also been observed by XPS [136] although, given the precision of the measurement, this band is broadened out and appears to extend into the oxygen $2 p$ valence band, the distinction of which is not made by the authors. Calculations using the local-density approximation and full- 
potential linear muffin-tin orbitals (where all electrons are considered, not just the valence electrons as in the case of the VASP program) result in density of states spectra which are virtually identical to those we have obtained $[58,114]$. This is the case in both the cubic and hexagonal systems. An older paper by D.W. Bullet [35], utilising a non-relativistic atomic orbital-based method shows a very similar band structure (oxygen $2 p$ as valence band and tungsten $5 d$ as conduction band).

Figure 4.5 shows a comparison of the density of states of the cubic bronzes with the parent oxide. The hydrogen bronze system shows a large peak feature at the bottom of the conduction band, which is attributed to the nonionised hydrogen 1s orbital. Because only one electron is contributed from the hydrogen, the Fermi level lies about in the middle of this sub-band. For the other bronze systems, analysis of the density of states contribution from each atom reveals that the intercalated atom contributes very little if at all to the overall density of states. This is expected due to its complete ionisation, as the single valence electron of the intercalated atom is contributed to the $\mathrm{W}-\mathrm{O}$ framework [35]. All compounds have the Fermi level located well into the conduction band, rendering them metallic. The shape of the band structure does not change as atoms are intercalated. This is also noted in other literature [58, 114]. Also, the magnitude of the band gap and the position of the Fermi level is relatively constant - even for those compounds which are known to be unstable (cubic potassium, rubidium and caesium bronzes).

We have also derived the band structure curves along lines of symmetry in cubic $\mathrm{WO}_{3}$ and cubic $\mathrm{NaWO}_{3}$. These are compared in Figure 4.6 with the results of Bullett [35] and Corà et al. [58] and agree extremely well with both of these, considering that different methods were used for each calculation. We again notice the high degree of similarity between the parent oxide and the sodium bronze, with the only major difference being the position of the Fermi level within a more or less rigid band structure. 


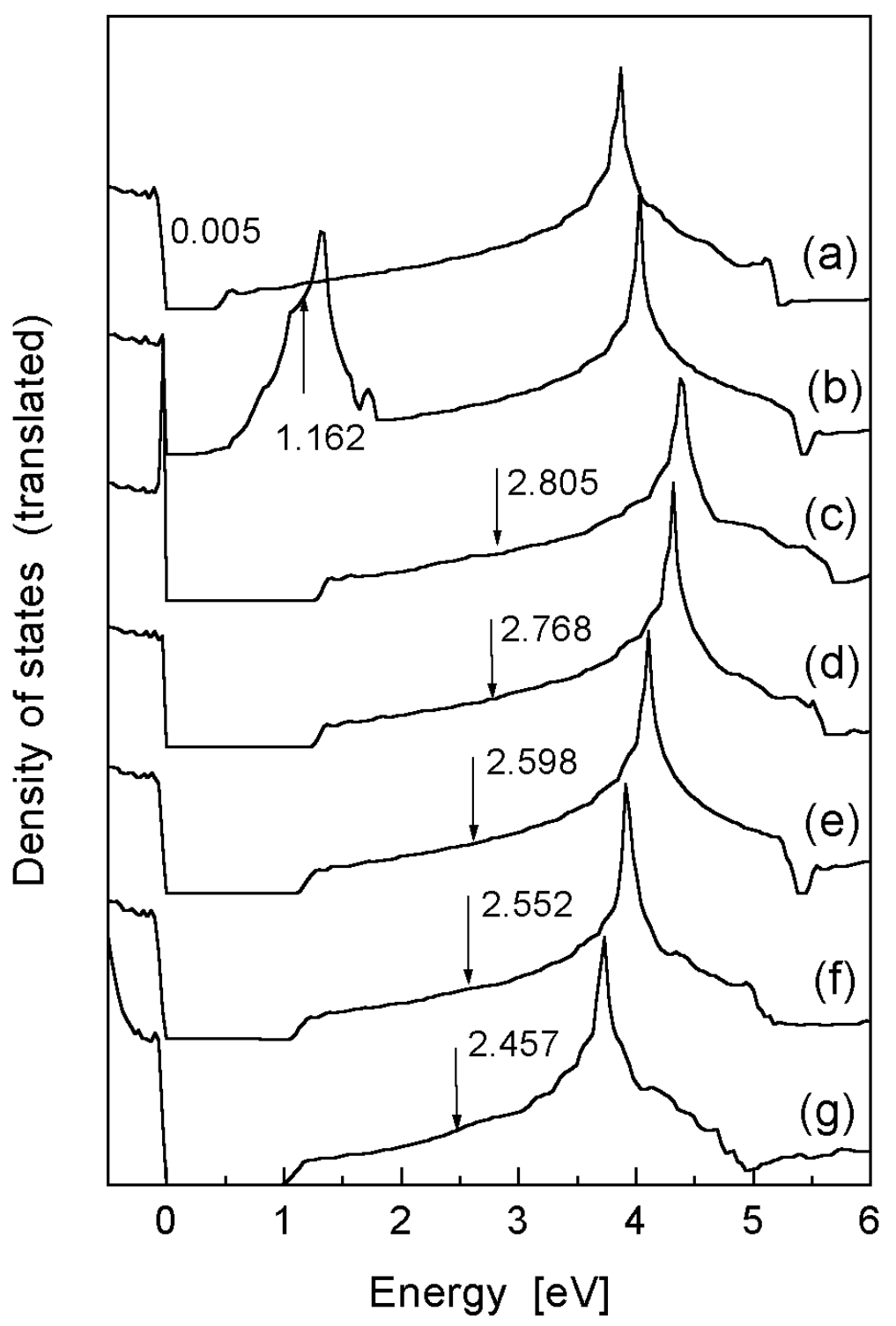

Figure 4.5: Calculated density of states for cubic tungsten bronzes, $\mathrm{MWO}_{3}$, near the Fermi level: (a) $\mathrm{WO}_{3}$, (b) $\mathrm{HWO}_{3}$, (c) $\mathrm{LiWO}_{3}$, (d) $\mathrm{NaWO}_{3}$, (e) $\mathrm{KWO}_{3}$, (f) $\mathrm{RbWO}_{3}$, (g) $\mathrm{CsWO}_{3}$. The Fermi level is indicated in each case. 

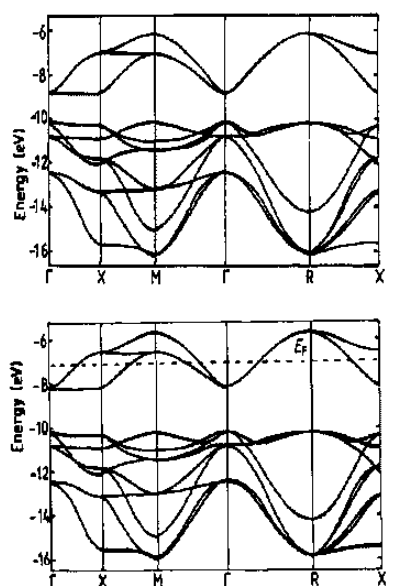

a
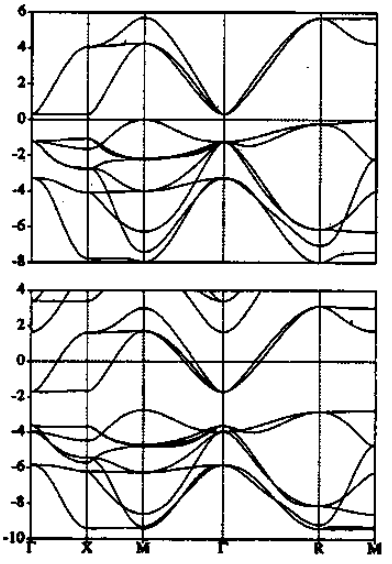

b
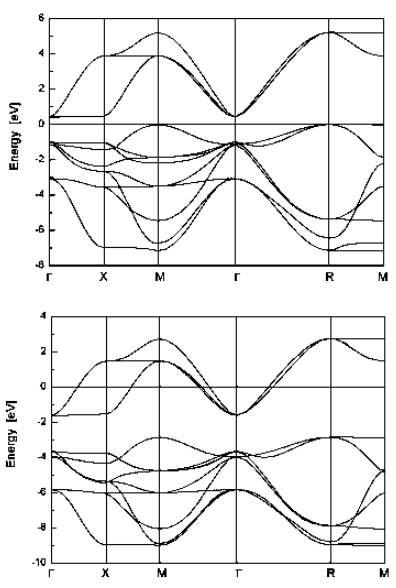

$\mathrm{c}$

Figure 4.6: Band structure diagrams of cubic $\mathrm{WO}_{3}$ (top) and $\mathrm{NaWO}_{3}$ (bottom). The results of (a) Bullett [35] and (b) Corà et al. [58] are compared with our results (c).

\subsection{Cubic Sodium Tungsten Bronze Series}

Following the work comparing cubic and hexagonal tungsten bronzes, we set out to explore the sodium bronze system more thoroughly. Experimentally it is quite difficult to obtain a completely saturated sodium tungsten bronze (i.e. $x=1$ ), and even when an excess of sodium tungstate is used in the reaction

$$
3 x \cdot \mathrm{Na}_{2} \mathrm{WO}_{4}+(6-4 \mathrm{x}) \mathrm{WO}_{3}+\mathrm{x} \cdot \mathrm{W} \rightarrow 6 \mathrm{Na}_{\mathrm{x}} \mathrm{WO}_{3}
$$

the resulting bronze may not achieve $x=1$ [33]. There is a raft of experimental results, however, on sodium bronzes with $x<1$ (refs. [14, 32, 33, 89, 255], to list but a few), all illustrating that $x$ is a continuous quantity and not confined to any series of exactly stoichiometric compounds (staging). In addition to $x=0$ and 1 , which were performed as part of the cubic tungsten oxide and alkali bronze series, we have calculated the structure and density of states for $x=\frac{n}{8} ; n=1-8$. The average cubic cell parameter was found to increase linearly with $x$ as shown in figure 4.7. Also shown in this figure is a series of reported experimental results (from [33] and the ICDD database). For higher $x$ contents the two data sets are very close, with the calculated pa- 


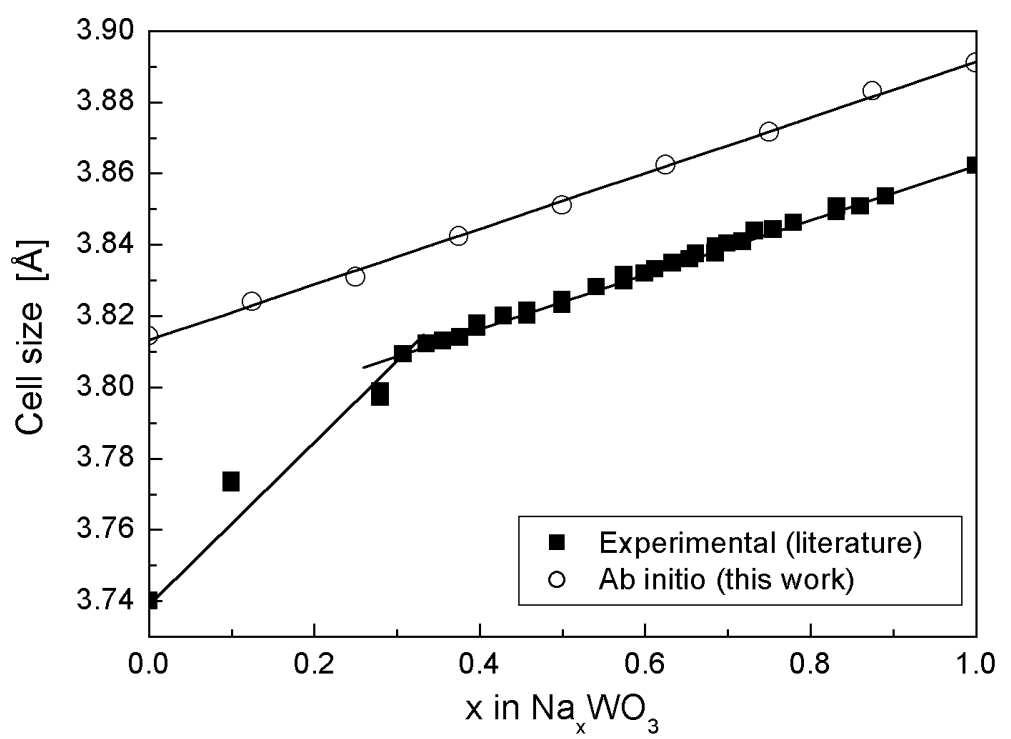

Figure 4.7: Calculated and experimental values of the crystal lattice cell parameter for cubic $\mathrm{Na}_{x} \mathrm{WO}_{3}$ with variable $x$.

rameters being about $0.8 \%$ larger than the experimental; again, a good result given the approximations made using this method. However below $x=0.3$, the experimental results differ markedly from the calculated values, due to a phase change to a tetragonal form at low $x$ values in the experimental system [31]. The structure of the tetragonal phase is a distorted version of the cubic phase [14]. As for the undoped $\mathrm{WO}_{3}$ system, such distortions are not accounted for in the current calculations. In the work of Walkingshaw et al. [271] the volume versus $x$ deviates from this linear behaviour for $x$ as large as 0.5 .

There are no obvious changes in the appearance of the density of states as $x$ increases from zero to one. The Fermi level and band edge are shown in figure 4.8. The band gap increases linearly with $x$, while the Fermi level quickly moves into the conduction band. According to this plot we would expect to see a semiconductor-metal transition at about $x=0.06$, where the Fermi level moves into the conduction band. However, in the experimental system this transition is observed at $x=0.3$, corresponding to (and perhaps influenced by) the structural transition (see [14], and references therein).

Once again, analysis of the individual atomic contributions to the density 


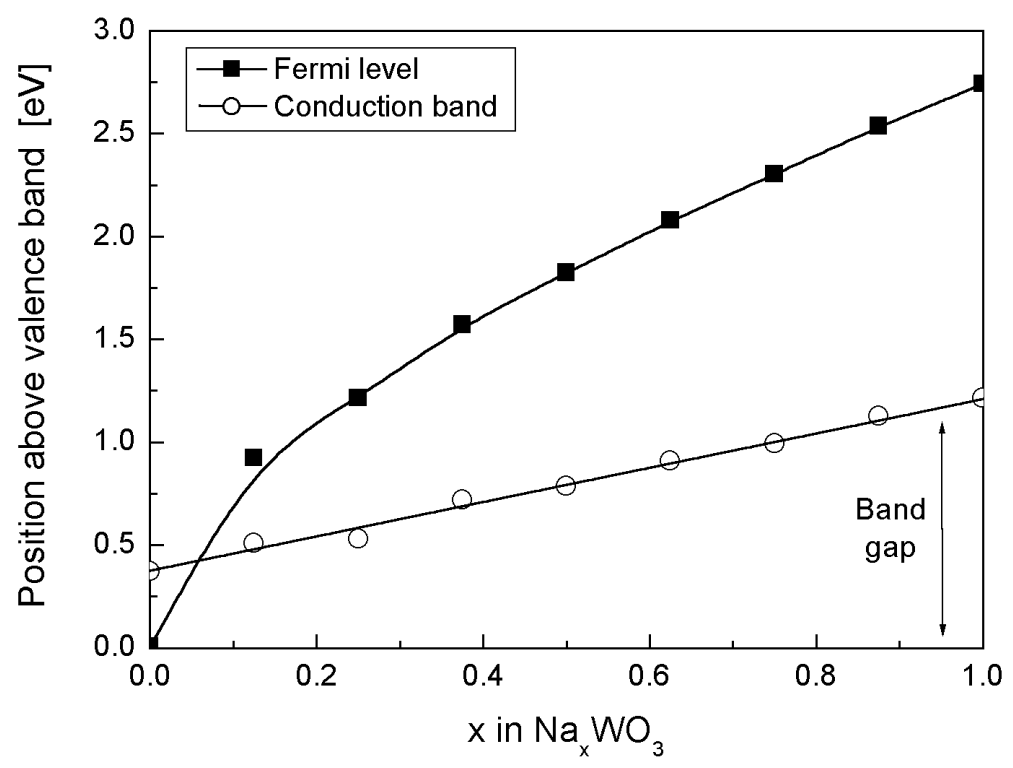

Figure 4.8: Evolution of the conduction band edge and Fermi level in $\mathrm{Na}_{x} \mathrm{WO}_{3}$, showing the movement of the Fermi level into the conduction band at low $x$ values. The lines are given as a guide.

of states indicates that each sodium atom is fully ionised, and the electron donated to the tungsten $5 d$ conduction band [35].

\subsection{Sub-stoichiometric $\mathrm{WO}_{3-x}$}

As a complement to the sodium bronze series, sub-stoichiometric $\mathrm{WO}_{3-x}$ was examined for compounds with $x$ ranging from zero to 0.33 . In the experimental system, the maximum oxygen loss that can be achieved without a drastic phase change is $\sim 0.35$ ([233], and references therein). The structure appears to 'stage' into distinct stoichiometric compositions for compounds in the range $\mathrm{WO}_{2.65}-\mathrm{WO}_{3}$, and for some of these the crystal structure has been solved. They are assigned the names $\alpha$-, $\beta$ - and $\gamma$-phase, as shown in Table 4.5 with their respective formulae. The crystal structures of the $\beta$ and $\gamma$-phases were solved by Magneli $[188,187]$. Booth et al. generalise the $\beta$-phase even further by describing the existence of crystallographic shear planes [29]. This can account for the broad range of compositional formulae. While we are unable to calculate the properties of these phases as described 


\begin{tabular}{cccc}
\hline Phase & Formula & Range & Average \\
\hline \hline$\alpha$ & $\mathrm{WO}_{3}$ & $\mathrm{WO}_{2.95}-\mathrm{WO}_{3}$ & $\mathrm{WO}_{3}$ \\
$\beta$ & $\mathrm{W}_{20} \mathrm{O}_{58}$ & $\mathrm{WO}_{2.88}-\mathrm{WO}_{2.94}$ & $\mathrm{WO}_{2.90}$ \\
$\gamma$ & $\mathrm{W}_{18} \mathrm{O}_{49}$ & $\mathrm{WO}_{2.65}-\mathrm{WO}_{2.76}$ & $\mathrm{WO}_{2.72}$ \\
$\delta$ & $\mathrm{WO}_{2}$ & $\mathrm{WO}_{1.99}-\mathrm{WO}_{2.02}$ & $\mathrm{WO}_{2}$ \\
\hline
\end{tabular}

Table 4.5: Compositional ranges for sub-stoichiometric tungsten oxide species. From [233].

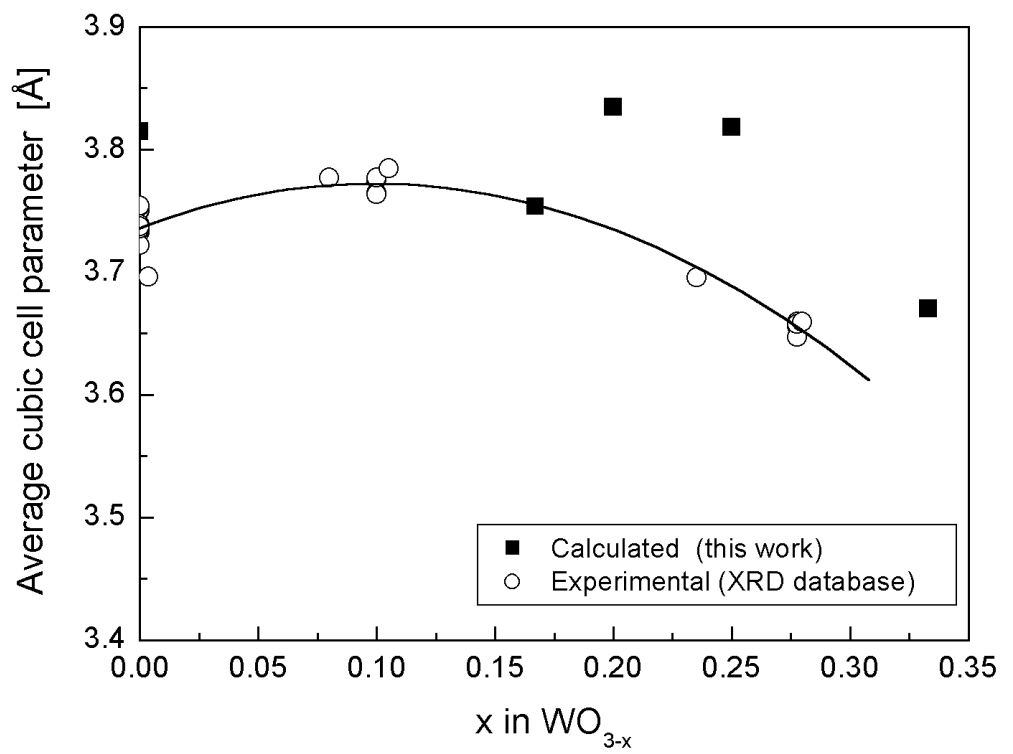

Figure 4.9: The volume-averaged cubic cell parameter for calculated and experimental sub-stoichiometric 'cubic' $\mathrm{WO}_{3-x}$ systems. The curve is given as a guide.

in literature due to the restriction on the number of atoms in the system, we are able to observe the effect that removal of oxygen has on the simple cubic $\mathrm{WO}_{3}$ phase.

As one might expect, removing oxygen from a site causes a local distortion of atoms around the vacancy, and the cell ceases to be simple orthorhombic. The cell volume results are shown in Figure 4.9 and are in good agreement with experimental values, despite the absence of the phase change in the calculated system.

The energies of formation for the different species studied are given in Table 4.6. We note that a slight deficit of oxygen $\left(x=\frac{1}{6}\right)$ is more favourable 


\begin{tabular}{cc}
\hline Formula & Energy of formation (eV/unit formula) \\
\hline \hline $\mathrm{WO}_{3}(x=0)$ & 0 \\
$\mathrm{WO}_{2.833}\left(x=\frac{1}{6}\right)$ & -0.103 \\
$\mathrm{WO}_{2.8}\left(x=\frac{1}{5}\right)$ & 0.447 \\
$\mathrm{WO}_{2.75}\left(x=\frac{1}{4}\right)$ & 1.007 \\
$\mathrm{WO}_{2.667}\left(x=\frac{1}{3}\right)$ & 1.661 \\
\hline
\end{tabular}

Table 4.6: Energies of formation of the calculated $\mathrm{WO}_{3-x}$ species calculated by the formula $E_{F}=E_{T O T A L}-\sum E_{P A R T S}=E\left(W O_{3-x}\right)-\left(E\left(W O_{3}\right)-\right.$ $\left.\frac{x}{2} E\left(O_{2}\right)\right)$.

energetically than stoichiometric $\mathrm{WO}_{3}$. This is observed experimentally, as commercial $\mathrm{WO}_{3}$ powder exhibits a loss of oxygen over 1-2 days in air. Further loss of oxygen renders $\mathrm{WO}_{3-x}$ less energetically favourable than its parent oxide, and once again, as was the case with sodium tungsten bronzes, the presence of a phase change in the experimental system may explain any discrepancies seen.

It is also of interest to look at the changes in the density of states as oxygen is removed from $\mathrm{WO}_{3}$. As mentioned in the introduction, oxygendeficient $\mathrm{WO}_{3}$ exhibits an increased conductivity, but not as great as that due solely to the presence of doped electrons. Figure 4.10 shows the density of states for the $\mathrm{WO}_{3-x}$ system in the region near the Fermi level. The overall spectra share the same features previously detailed for $\mathrm{WO}_{3}$ : the oxygen $2 \mathrm{~s}$ band near $-18 \mathrm{eV}$; the broad valence band, comprised mainly of oxygen $2 p$ orbitals, from -7 to $0 \mathrm{eV}$; and the conduction band, consisting solely of tungsten $5 d$ orbitals, lying from roughly 0.5 to $5 \mathrm{eV}$. Naturally the sub-stoichiometric systems appear more 'jagged' than the parent $\mathrm{WO}_{3}$ compound, due in part to the breaking of symmetry, rendering each atom non-equivalent to others within the cell, and causing its contribution to be slightly different.

The inset of Figure 4.10 shows the progression of the Fermi level into the conduction band. There is a sharp jump between $x=0.167$ and 0.2 as the Fermi level moves up into the conduction band - not a gradual transition as in the case of the sodium bronzes $\mathrm{Na}_{x} \mathrm{WO}_{3}$. While the $x=0.167$ compound has the Fermi level at zero (and therefore, still non-conducting), the band 


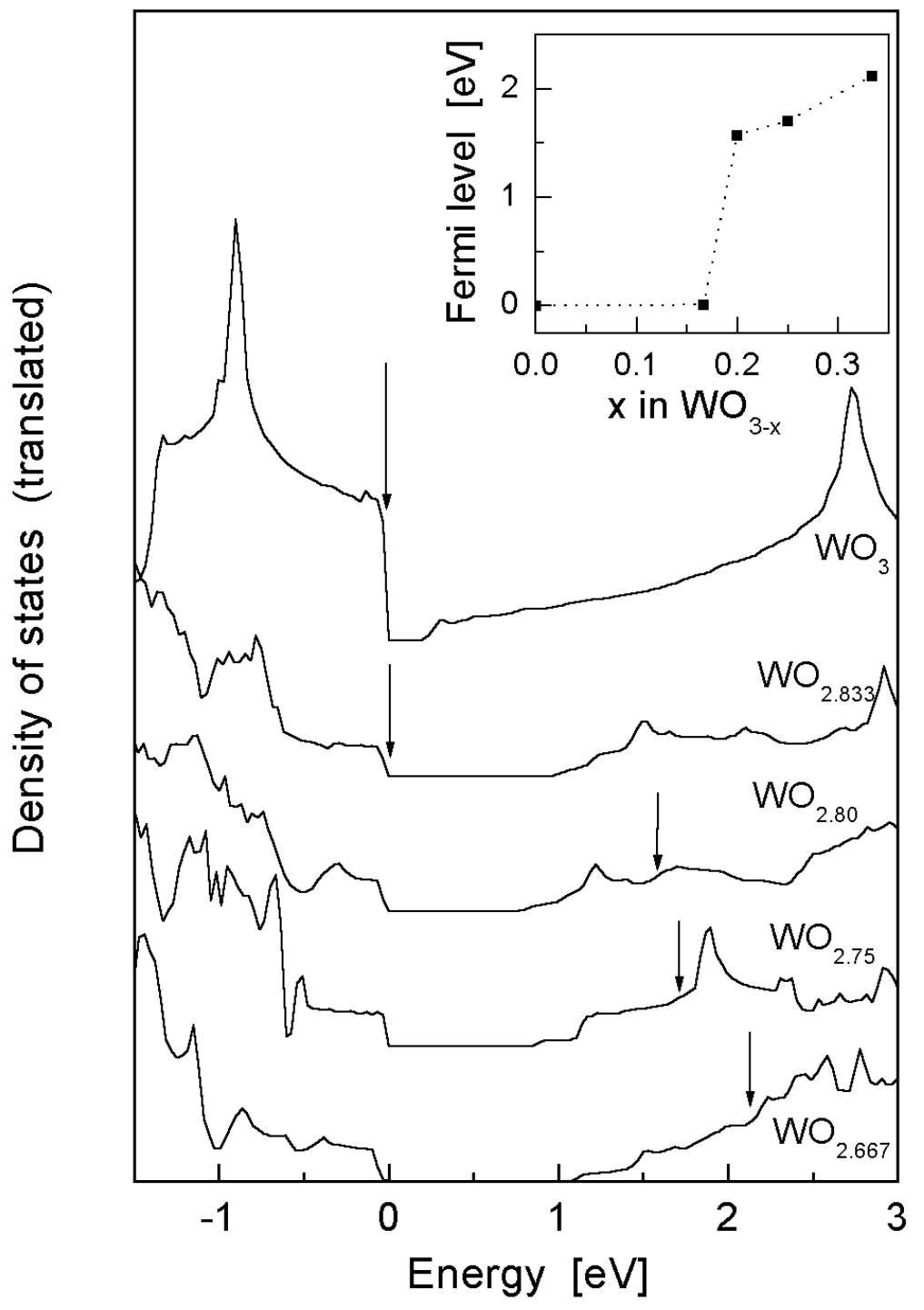

Figure 4.10: Density of states for the $\mathrm{WO}_{3-x}$ system, all with the valence band set at zero. Arrows show the position of the Fermi level. Inset: Position of the Fermi level relative to the top of the valence band. 
structure of the valence band is similar to that of the conducting species. It appears that there is a decrease in the density of states in the valence band, followed by the Fermi level being pushed up into the conduction band. The stoichiometry at which this insulating-conducting transition occurs is in good agreement with the literature value of $\mathrm{WO}_{2.76}$, which coincides with the $\beta-\gamma$ structural phase transition [95].

\subsection{Organic-Inorganic Layered Hybrids}

Following on from the background studies of tungsten bronzes and the oxygendeficient tungsten oxides, layered organic-inorganic hybrid compounds were studied. Three different length alkyl amines were used in the calculations, with two, four and six carbons. The input structure of the hybrid systems is the most conceptually simple: a single unit formula, $\mathrm{WO}_{4} \cdot \mathrm{H}_{3} \mathrm{~N}\left(\mathrm{CH}_{2}\right)_{n} \mathrm{NH}_{3}$ $(n=2,4,6)$. It is highly conceivable that the calculated structure of the hybrid compounds is in a slightly higher energy state than that of the actual structure, which may be a supercell of the simple input case, with possible tilts and/or rotations of the octahedra and organic molecules. As mentioned, $\mathrm{WO}_{3}$ does not form a simple cubic structure, but exhibits small distortions of bond lengths and angles which render it very slightly off-cubic, with eight formula units per cell. In extending the computations to the organic-inorganic systems then, several constraints were necessary. Firstly only one cell was used, which may affect the outcome not only due to the removal of distortion in the inorganic layer (which apparently lowers the energy in the oxide and hydrate compounds) but also because this does not allow for the differing orientation of the organic molecules in neighbouring cells. Secondly, constraints were placed on the inorganic atoms in order to maintain the position of the layer. This entailed fixing the position of the tungsten and planar oxygen atoms at the corner and edges of the cell respectively. This was sufficient to relax the atoms to a sensible structure. 

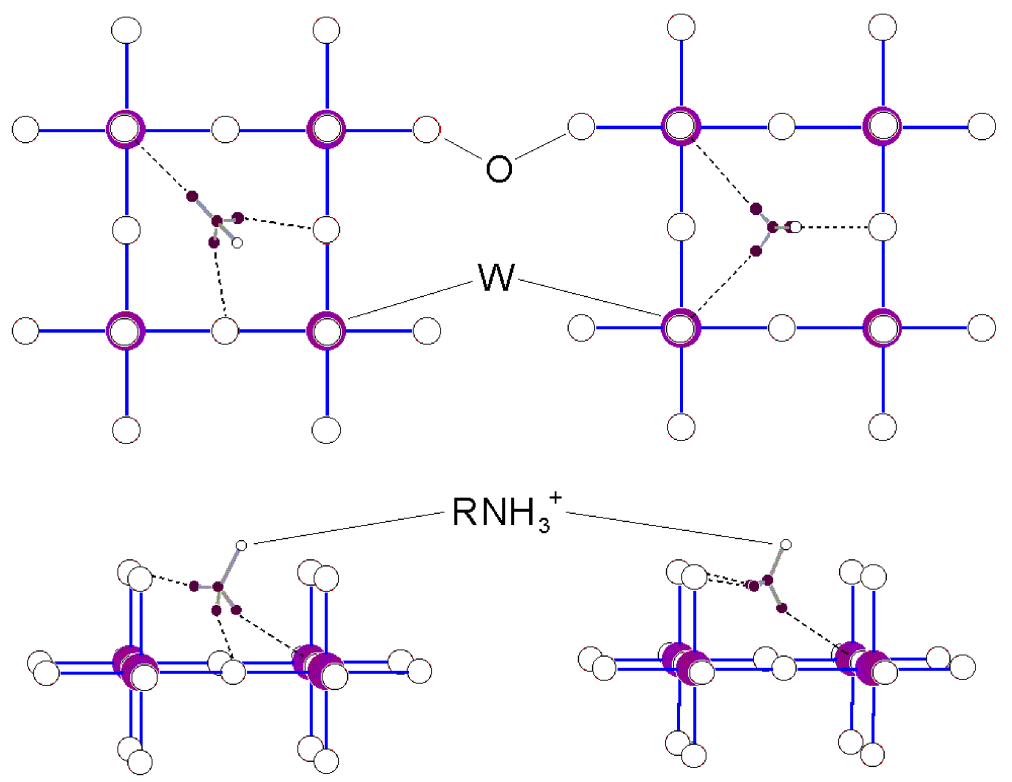

Figure 4.11: Schematic diagrams illustrating the two bonding configurations, 'bridging' (left) and 'apical' (right), in organic-inorganic hybrid materials. Only the first carbon atom of the organic molecule is shown for simplicity.

\subsubsection{Structure}

In the possibly isostructural metal halide hybrid systems, two schemes were identified in reference [196] for the bonding of the organic ammonium group to the inorganic layer, designated 'bridging' and 'terminal' (which we shall call 'apical'). In the 'bridging' case the organic ammonium forms hydrogen bonds to two bridging and one apical atom while in the 'apical' case hydrogen bonds are formed to two apical and one bridging atom (Figure 4.11). This causes the alkyl chain to lie diagonally within the cell when the ammonium group is in a bridging configuration, but parallel for the apical. When the alkyl chain is longer than one carbon (methylamine), the second carbon in the chain would be too close to the opposing apical (oxygen) atom if a bridging conformation were adopted. Thus in general, apical bonding is observed for organic-inorganic systems with organic chain lengths of two or more carbon atoms.

Given this, calculations were initially performed on the methylamine system in both the bridging and apical configurations, to compare the energies 


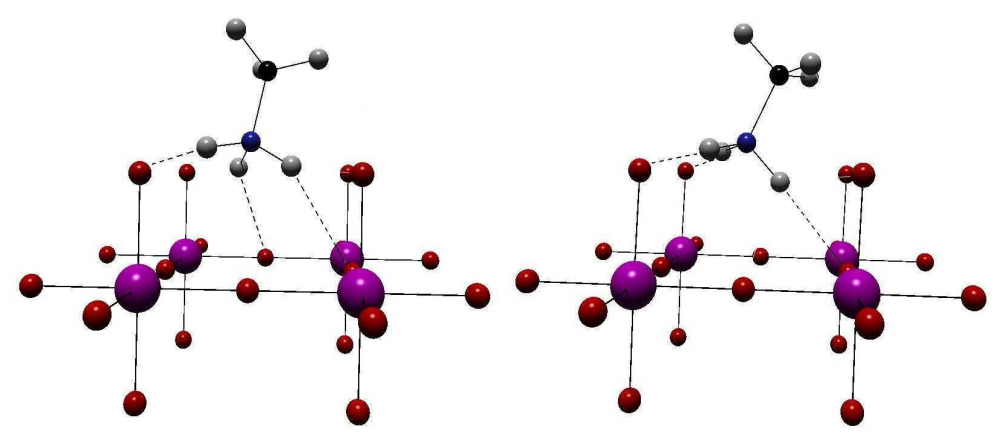

Figure 4.12: Calculated structure of methylamine in bridging (left) and apical (right) configurations.

of each and as a starting point to construct the initial cells for the computations of the longer chain diamine hybrids. Figure 4.12 shows the calculated relaxed structures of one methylammonium ion with respect to the tungsten oxide layer. In both cases the calculated structure closely resembles that expected from the diagram in Figure 4.11. In the apical case the shortest hydrogen bonds between the ammonium hydrogen atoms and the oxygen atoms of the inorganic layer are as expected: two short hydrogen bonds to two apical oxygens and one to the opposing bridging oxygen. However, for the bridging case the two hydrogens that were expected to interact with adjacent bridging oxygens are actually closer to apical oxygens. In this latter case the tilting of the organic molecule is much less than for the apical case, and overall it appears that there is a more delocalised attraction between the hydrogen and oxygen atoms.

The energies of the two systems were calculated to be $-123.31 \mathrm{eV}$ for the apical case and $-123.08 \mathrm{eV}$ for the bridging. Thus the apical configuration appears to be more stable, both from its lower energy and examination of the structure with more localised forces. Each of the two methylamine structures were used as a basis for the initial positions of the diaminoethane (DA2) compound. The relaxed cell parameters are given in Table 4.7. In the apical case the cell volume is slightly less than in the bridging case. Despite the greater tilting expected for the apical structure, the planar axes ( $a$ and $c$ ) are shorter and the interlayer spacing $b$ is longer than for the bridging. The two structures are shown in Figure 4.13. As for the methylammonium structures, 


\begin{tabular}{lcc}
\hline & W-DA2 bridging & W-DA2 apical \\
\hline \hline $\mathrm{a}(\AA)$ & 3.9443 & 3.9108 \\
$\mathrm{~b}(\AA)$ & 8.7345 & 8.7992 \\
$\mathrm{c}(\AA)$ & 3.9443 & 3.9245 \\
$\alpha$ & 89.98 & 89.99 \\
$\beta$ & 90.02 & 90.01 \\
$\gamma$ & 90.02 & 90.01 \\
Volume $\left(\AA^{3}\right)$ & 135.8266 & 135.0498 \\
\hline
\end{tabular}

Table 4.7: Calculated cell parameters of W-DA2 with the organic molecule in the bridging and apical conformations.

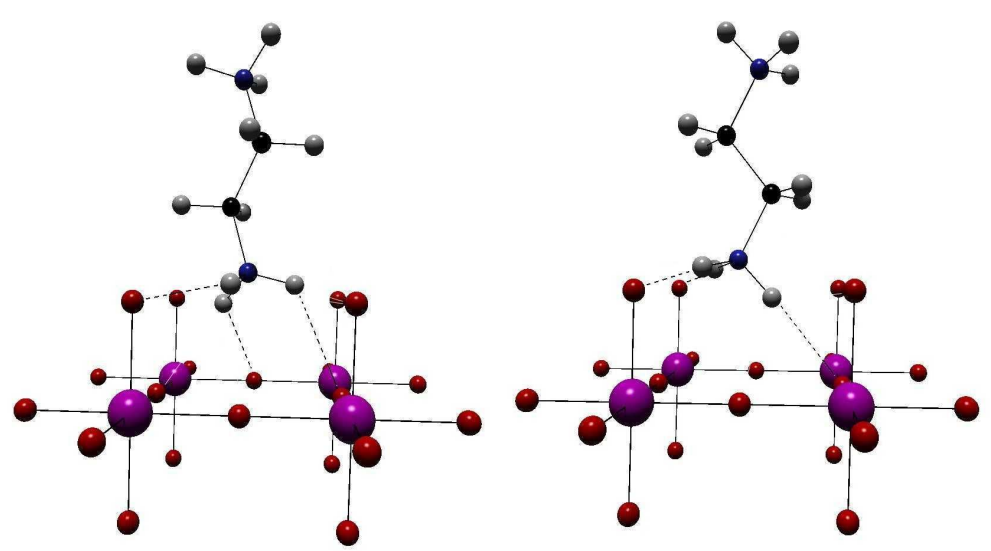

Figure 4.13: Calculated structures of diaminoethane (DA2) in bridging (left) and apical (right) configurations.

in the bridging conformation there are several longer bond distances from each hydrogen to the oxygen atoms, whereas in the apical conformation for each hydrogen there is a single bond that is distinctly shorter to one oxygen than the others.

The energies of these two structures are calculated to be $-114.709 \mathrm{eV}$ for the bridging conformation and $-115.750 \mathrm{eV}$ for the apical. The apical conformation is therefore again more stable, and the difference between the two is greater than for the methylammonium case. Two other systems were extended from the apical W-DA2: W-DA4 (4-carbon chain) and W-DA6 (6carbon chain). Again the planar tungsten and oxygen atom positions were fixed. The energies of formation are given in Table 4.8. As can be seen 


\begin{tabular}{clll}
\hline Compound & $\begin{array}{l}\text { Ground state en- } \\
\text { ergy }(\mathrm{eV})\end{array}$ & $\begin{array}{l}\text { Ground state en- } \\
\text { ergy of organic } \\
\text { molecule }(\mathrm{eV})\end{array}$ & $\begin{array}{l}\text { Energy of forma- } \\
\text { tion }(\mathrm{eV})\end{array}$ \\
\hline \hline W-DA2 & -115.750 & -64.172 & -0.939 \\
W-DA4 & -148.189 & -97.506 & -0.044 \\
W-DA6 & -181.887 & -130.714 & -0.534 \\
\hline
\end{tabular}

Table 4.8: Energies of formation of the calculated W-DAn compounds, calculated by the formula $E_{F}=E_{\text {TOTAL }}-\sum E_{\text {PARTS }}=E\left(W O_{4}\right.$. $\left.\mathrm{H}_{3} \mathrm{~N}\left(\mathrm{CH}_{2}\right)_{n} \mathrm{NH}_{3}\right)-\left(\mathrm{E}\left(\mathrm{H}_{2} \mathrm{WO}_{4}\right)+E\left(\mathrm{H}_{2} \mathrm{~N}\left(\mathrm{CH}_{2}\right)_{n} \mathrm{NH} \mathrm{H}_{2}\right)\right)$.

from the negative values, all three compounds are stable, with W-DA4 being the least stable of the three. This seems to be confirmed experimentally as W-DA4 is harder to form than both W-DA2 and W-DA6.

\subsubsection{Density of states}

The density of states of the three compounds are all very similar. The results are shown in Figure 4.14. The main features are as follows: As in the tungsten oxide and tungsten bronze systems, the oxygen $2 \mathrm{~s}$ band is located between -18 and $-16 \mathrm{eV}$. There is a splitting between the planar and apical oxygen contributions, with the planar oxygen bands being broader and lying at slightly lower energies. The nitrogen $2 \mathrm{~s}$ bands lie at about $-18.5 \mathrm{eV}$ and the carbon $2 \mathrm{~s}$ bands lie between -16 and $-9 \mathrm{eV}$. The appearance of multiple carbon s bands in the longer chain systems is due to the different environments in which the carbon atoms are located along the length of the chain. Between -9 and $0 \mathrm{eV}$ lie the $2 p$ bands of $\mathrm{N}$ (lowest), $\mathrm{C}$ (middle) and $\mathrm{O}$ (highest). The hydrogen atoms associated with the carbon and nitrogen atoms contribute their single 1 s electrons to the bands of their respective atoms.

The oxygen band, from -2 to $0 \mathrm{eV}$, closely resembles that of the tungsten oxides and bronzes. There is relatively little organic or tungsten component to this band, so once again the valence band is comprised of oxygen $2 p$ orbitals. The conduction band begins at around $0.4 \mathrm{eV}$ but the density of states is very low up to a peak feature at about $2.7 \mathrm{eV}$. There is also a second peak feature at $4.0 \mathrm{eV}$. This band is comprised mostly of tungsten $5 \mathrm{~d}$ orbitals, 


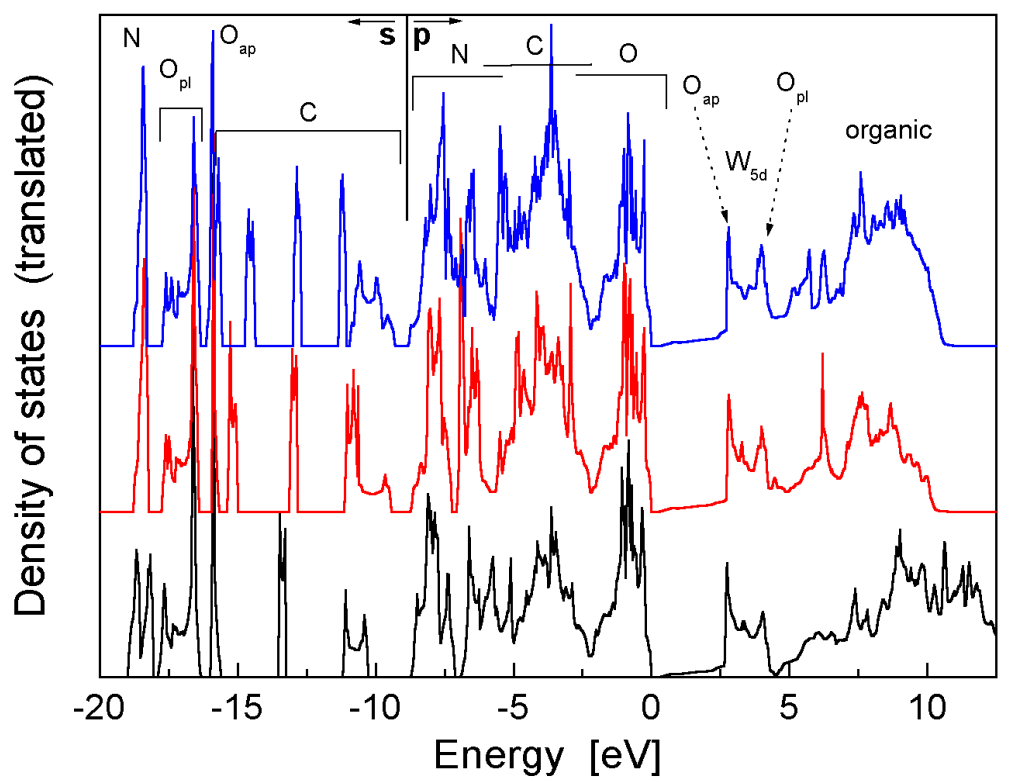

Figure 4.14: Calculated density of states for W-DA2 (bottom), W-DA4 (middle) and W-DA6 (top). The Fermi level is located at $\mathrm{E}=0$.

as in the tungsten oxides and bronzes, but in these hybrid compounds there is an additional oxygen $2 p$ component to this band. The apical oxygen atom contributes to the first peak feature and the planar oxygen atoms contribute to the second. Above $4.5 \mathrm{eV}$ lie the organic anti-bonding orbitals. The band structure in the vicinity of the Fermi level (i.e. valence and conduction bands) is virtually identical for the three different organic intercalates. As expected the organic molecule does not participate in electronic conduction and the undoped compound is an insulator. These results are now compared with UV-visible spectroscopy.

\subsection{UV-visible spectroscopy}

First we describe and discuss the diffuse reflectance powder results, and then the transmittance and reflectance analysis of thin film samples. 


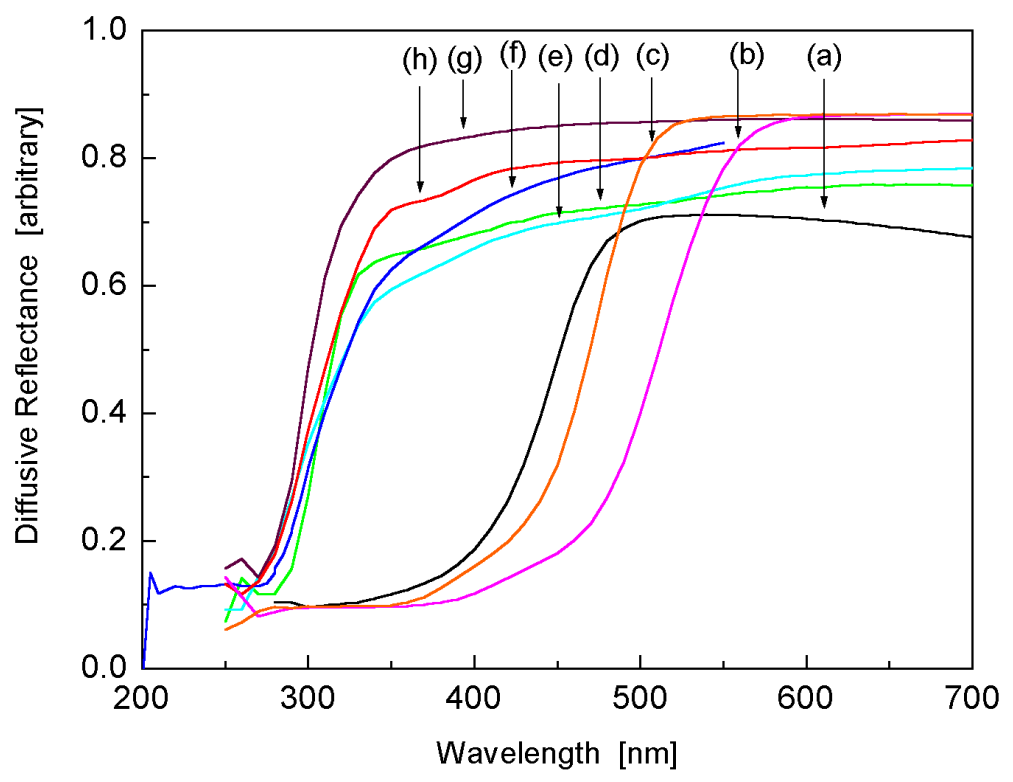

Figure 4.15: UV-visible spectra of tungsten oxide-based materials: (a) $\mathrm{WO}_{3}$; (b) $\mathrm{H}_{2} \mathrm{WO}_{4}$; (c) $\mathrm{WO}_{3} \cdot 2 \mathrm{H}_{2} \mathrm{O}$; (d) W-DA2; (e) W-DA4; (f) W-DA6; (g) WDA8; (h) W-DA12.

\subsubsection{Powder diffuse reflectance}

Powder diffuse reflectance was performed on samples as detailed in Section 2.4. Figure 4.15 shows the diffuse reflectance of $\mathrm{WO}_{3}, \mathrm{H}_{2} \mathrm{WO}_{4}\left(\equiv \mathrm{WO}_{3} \cdot \mathrm{H}_{2} \mathrm{O}\right)$, $\mathrm{WO}_{3} \cdot 2 \mathrm{H}_{2} \mathrm{O}$, and the W-DAn hybrid series $(n=2,4,6,8,12)$. In each case there is a single absorption edge, the midpoints of which are given in Table 4.9 .

There is no apparent trend in the position of the absorption edge among the W-DAn samples, suggesting that the inorganic planes are more or less completely electronically decoupled. However, their respective energies are significantly higher compared with $\mathrm{WO}_{3}$ and its inorganic layered hydrates, $\mathrm{WO}_{3} \cdot n \mathrm{H}_{2} \mathrm{O}$. As mentioned in Section $1.3, \mathrm{WO}_{3}$ has been reported to have an indirect band gap of $\sim 2.6 \mathrm{eV}[58,100,172]$, although band gaps of up to $3.2 \mathrm{eV}$ have been reported for thin films [19, 93, 225]. The obtained value here of $2.8 \mathrm{eV}$ is comparable with the common value of $2.6 \mathrm{eV}$.

As mentioned in Section 4.3.4, peak features in the density of states, as well as band gaps, may be the cause of transitions observed in optical 


\begin{tabular}{ccc}
\hline Sample & Position $(\mathrm{nm})$ & Energy $(\mathrm{eV})$ \\
\hline \hline $\mathrm{WO}_{3}$ & 444 & 2.79 \\
$\mathrm{H}_{2} \mathrm{WO}_{4}$ & 494 & 2.51 \\
$\mathrm{WO}_{3} \cdot 2 \mathrm{H}_{2} \mathrm{O}$ & 473 & 2.62 \\
\hline $\mathrm{W}-\mathrm{DA} 2$ & 310 & 4.00 \\
$\mathrm{~W}-\mathrm{DA} 4$ & 311 & 3.99 \\
$\mathrm{~W}-\mathrm{DA} 6$ & 313 & 3.96 \\
W-DA8 & 297 & 4.17 \\
W-DA12 & 305 & 4.07 \\
\hline
\end{tabular}

Table 4.9: Absorption edge mid-point values of tungsten oxide-related materials diffuse reflectance UV-visible spectra.

spectra. Indeed, in the density of states of the hybrid materials (which are identical near the Fermi level and independent of the length of the organic intercalate) there are peak features at 2.7 and $4.0 \mathrm{eV}$. The optical transition may be related to the latter.

\subsubsection{Spectroscopy of thin films}

$\mathrm{WO}_{3}$ films produced by spin-coating and electron-beam evaporation (described in Section 2.4) display very similar absorption coefficients upon analysis of the reflectance and transmittance spectra [121]. This indicates that the same material has been formed by the two methods and that the films have similar degrees of crystallinity. Figure 4.16 shows that the absorption coefficient of $\mathrm{WO}_{3}$ films increases by about two orders of magnitude above 1 $\mathrm{eV}$ with the maximum value at $4.0 \mathrm{eV}$. In many other materials a feature like this is related to a direct band gap. In the calculated band structure of $\mathrm{WO}_{3}$ (Figure 4.6 on page 79 ) the direct band gap is clearly seen to be around 4 $\mathrm{eV}$, and corresponds to the position of the major peak feature in the density of states of $\mathrm{WO}_{3}$ (page 76).

Optical measurements on thin films of the hybrid materials were much more difficult than for the $\mathrm{WO}_{3}$ films above, due to the very close similarity in refractive index between the film and the substrate. $\mathrm{WO}_{3}$ has a refractive index of 2.5-3 [68], compared with quartz $\sim 1.5$. The hybrids are expected to have a refractive index of $\sim 1.3-1.6$. The closer the two refractive indices 


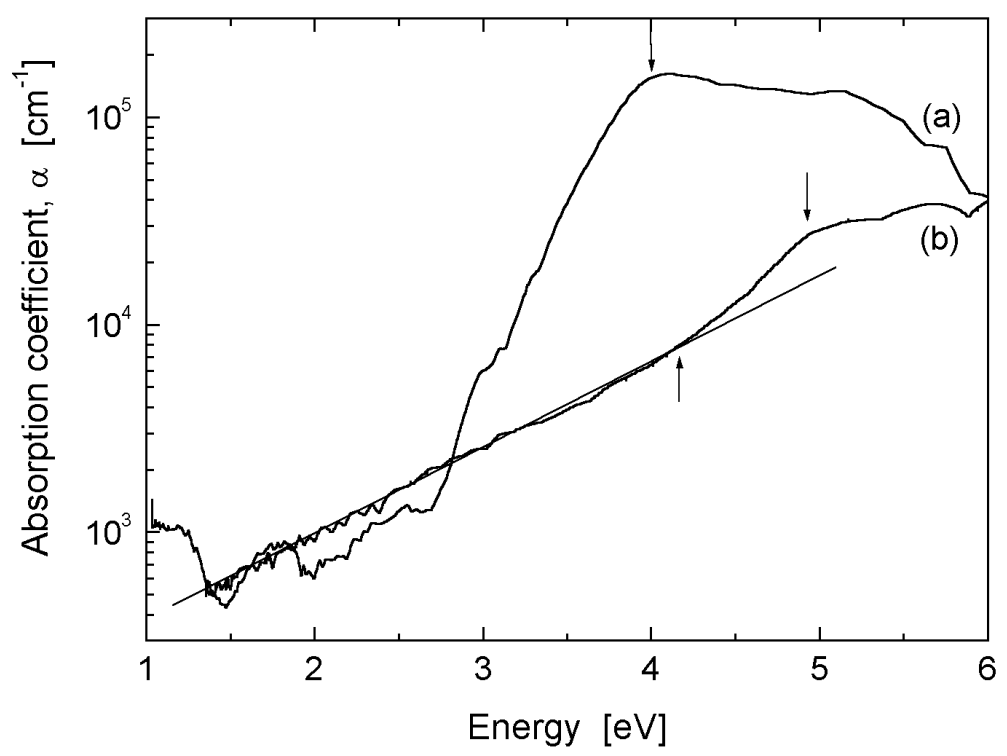

Figure 4.16: Absorption coefficients of (a) $\mathrm{WO}_{3}$ and (b) W-DA12 thin films, obtained from the reflectance and transmittance spectra. Arrows indicate changes in gradient, corresponding to band structure features (see text).

are, the less interference there is at the boundary between them, which is used to determine the absorption coefficient.

While analysis of most of the hybrid spectra showed no difference from a plain quartz substrate, a long-chain W-DA12 hybrid showed a steady increase in the absorption coefficient across the visible spectrum, with changes of gradient at 4.2 and $4.9 \mathrm{eV}$ (indicated by arrows in Figure 4.16). The refractive index is calculated to be $\sim 1.52$.

The calculated density of the states for the W-DAn hybrid materials (Section 4.6.2) exhibits two peak features at 2.7 and $4.0 \mathrm{eV}$. This latter peak may correspond to an indirect band gap as seen in the powder diffuse reflectance. The low density of states from $1 \mathrm{eV}$ up to the peak feature at $2.7 \mathrm{eV}$ may account for the steady increase at lower energies seen in the absorption coefficient plot.

While there may be a direct band gap at $4.9 \mathrm{eV}$ as indicated by the absorption coefficient analysis, it must be pointed out that these energies are close to the detection limits of the instrument, and that the calculated density of states is only valid for the very first few unoccupied bands. That 
is, in the hybrids case, one cannot say for certain that there is no direct band gap at $4.9 \mathrm{eV}$.

In summary, powder diffuse reflectance gives an indication of indirect band gaps, and absorption coefficient analysis of thin film reflectance and transmittance spectra gives an indication of direct band gaps. In $\mathrm{WO}_{3}$ these are found to be 2.8 and $4.0 \mathrm{eV}$ respectively, by both experiment and calculations. The W-DAn hybrids have identical band structures regardless of alkyl chain length (i.e. interlayer spacing). We assign an indirect band gap of 4.0-4.1 $\mathrm{eV}$ from powder diffuse reflectance to the peak feature observed in the calculated density of states of the same energy. Evidence is seen in the thin film absorption coefficient analysis for a direct band gap of $4.9 \mathrm{eV}$, however the calculated density of states cannot be relied upon up to this energy to comment.

In any case we see an increase of the band gap values, both direct and indirect, in going from the 3-dimensional $\mathrm{WO}_{3}$ to the 2-dimensional hybrid materials.

Recently, we have explored the doping of some of these hybrid materials using cyclic voltammetry (Chapter 7). It will be of particular interest as to whether one sees a build-up of low energy spectral weight with the development of free carrier density and the formation of a Drude peak. 


\section{Chapter 5}

\section{Transition Metal Hybrids}

Following from the structural and compositional determination of the 'parent' tungsten oxide hybrid species, several avenues were explored with regards to doping these materials. The addition of dopants, as seen in the computations of the tungsten oxide and tungsten bronzes, causes changes in the band structure and movement of the Fermi level into a partially occupied band, inducing conduction. This was a key objective in this research. In addition, we sought especially to introduce transition metal ions to establish strong electronic correlations. It is clear that a combination of low-dimensionality as well as strongly interacting electronic states will provide for the prospect of competing electronic phases and even the possibility of superconductivity. This was the primary reason for developing this class of layered hybrid material. Certainly, they will exhibit some degree of magnetic ordering.

The easiest way to introduce dopant species into the inorganic layer is to add such species to the solution during synthesis. Certain transition metals are known to have a variety of oxidation states and may easily accept or donate additional charge to the tungsten ions.

We synthesised several samples which included the transition metal ions $\mathrm{Mn}, \mathrm{Fe}, \mathrm{Co}, \mathrm{Ni}$ and $\mathrm{Cu}$ as detailed in Section 2.2. This chapter compares the synthesis and describes the characterisation of these new materials, and in Chapter 6 their interesting magnetic properties are discussed. 


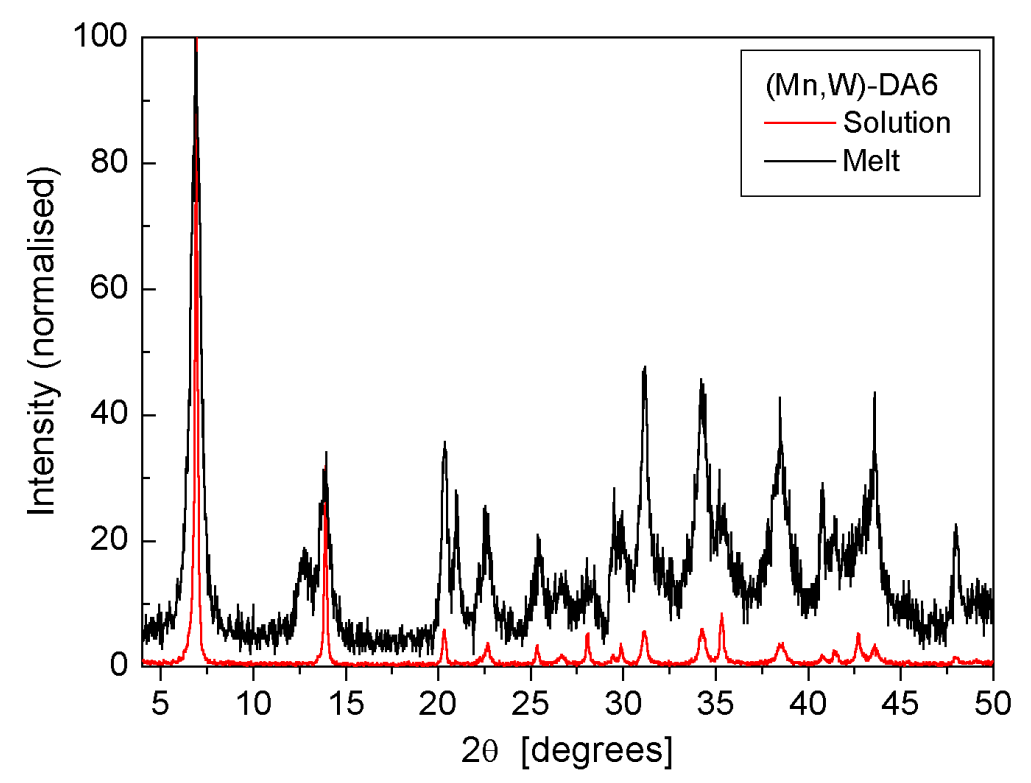

Figure 5.1: Normalised XRD patterns of two (Mn,W)-DA6 samples synthesised by the solution and melt methods.

\subsection{Synthesis}

As described in Chapter 3, three methods were used to synthesise the transition metal tungstate hybrids: the solution-based, 'melt', and Chimie douce methods.

Manganese and iron are the only two studied transition metal ions incorporated in the hybrids that are able to be formed by the standard $\mathrm{H}_{2} \mathrm{WO}_{4}$ /ammonia solution method. A (Mn,W)-DA6 sample was synthesised by the melt method from $\mathrm{MnWO}_{4} \cdot n \mathrm{H}_{2} \mathrm{O}$ and compared with the solution-produced sample, using XRD. The results are shown in Figure 5.1. There are a few additional peaks in the melt-produced sample (at 12.5 and $21^{\circ} 2 \theta$ ) but apart from this the positions of all the lines are virtually unchanged, indicating that the same phase has been formed.

While the crystal structure of $\mathrm{MnWO}_{4} \cdot n \mathrm{H}_{2} \mathrm{O}$ is not known, the structure of $\mathrm{MnWO}_{4}$ is, so a second experiment was undertaken to try to produce (Mn,W)-DA6 from a melt method using $\mathrm{MnWO}_{4}$ as the inorganic reagent. $\mathrm{XRD}$ of the final product revealed that the $\mathrm{MnWO}_{4}$ was unchanged. This is not surprising as, unlike the hydrate, $\mathrm{MnWO}_{4}$ has a closely-packed 3- 
dimensional structure [158] comprising interlocking chains of linked $\mathrm{MnO}_{6}$ and $\mathrm{WO}_{6}$ octahedra. Intercalation would require rupture of a large fraction of these strong bonds. Hence because the hybrid was formed, the hydrate materials are a valid starting material for synthesising other transition metal hybrids using the melt and chimie douce methods.

The exact crystal structure of the transition metal tungstate hydrates has not been reported, although there have been studies on a variety of their other structures formed at low $\mathrm{pH}$ [151] (see also ref. [55] in relation to $\mathrm{MnMoO}_{4} \cdot n \mathrm{H}_{2} \mathrm{O}$ ). As noted in Section 3.1.2, synthesis in low $\mathrm{pH}$ solutions encourages the formation of polyoxotungstate anions. The same is true of transition metal tungstate structures. Keggin ions can have one or more $\mathrm{WO}_{6}$ octahedra replaced by metal oxide octahedra [105, 183], or even new structures formed based on the 'caps' of the Keggin ion with a spacing 'ring' of the metal oxide species $[56,143,144]$. However these examples are for the most part synthesised at low $\mathrm{pH}$ and/or under extreme synthesis conditions (e.g. hydrothermally). Our hydrate samples were synthesised at room temperature in a $\mathrm{pH}$-neutral solution.

XRD patterns of the hydrate materials are amorphous, as shown by the broad features and low counts in Figure 5.2. This is in contrast to $\mathrm{H}_{2} \mathrm{WO}_{4}$, which is crystalline [145] (see, for example, Figure 7.4a). Their low (or nanocrystalline) crystallinity is also evident from the morphology displayed in SEM micrographs (Figure 5.3), which appear to show the material forms in clumps of smaller particles only a few hundred nanometres across.

The IR spectra (Figure 5.4) of these materials are similar - they display very few peaks, including a strong broad $\mathrm{O}-\mathrm{H}$ stretching mode (corresponding to the water molecules) centred at $\sim 3400 \mathrm{~cm}^{-1}$. According to ref. [170], the hydrates $\mathrm{MWO}_{4} \cdot n \mathrm{H}_{2} \mathrm{O}$ of the metal tungstates studied can have $n$ values of $2(\mathrm{M}=\mathrm{Mn}, \mathrm{Co}, \mathrm{Cu}), 3(\mathrm{M}=\mathrm{Fe}, \mathrm{Ni})$ or $6(\mathrm{M}=\mathrm{Ni})$, however the value of $n$ is often ill-defined, which may be related to the low crystallinity. 


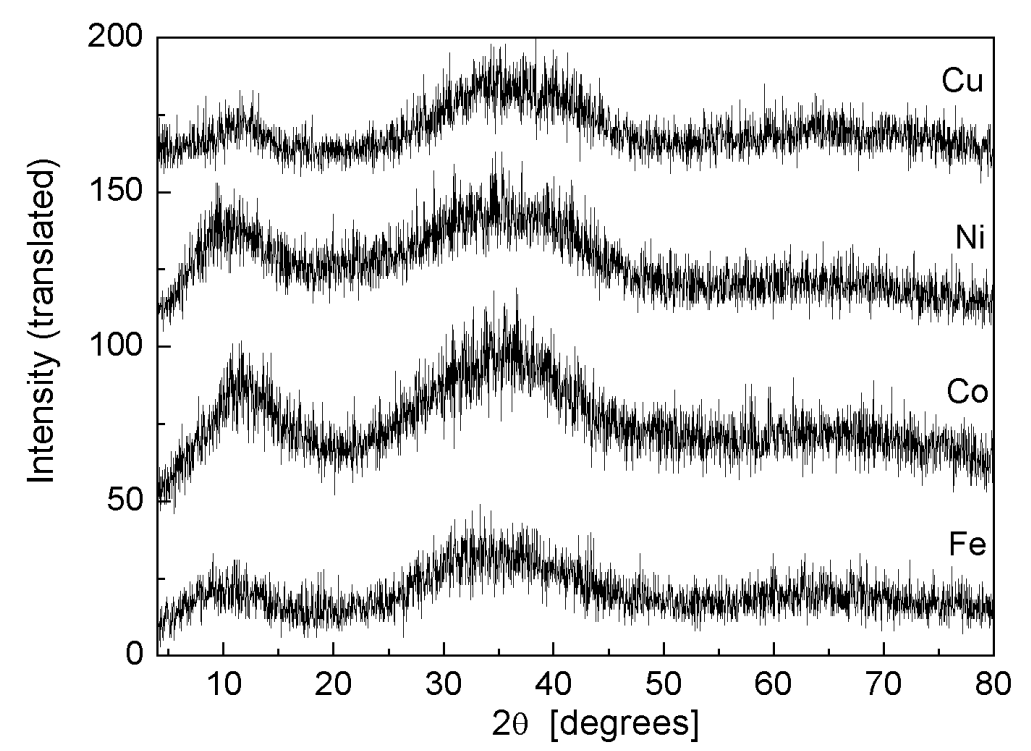

Figure 5.2: XRD patterns of transition metal tungstate hydrates $\left(\mathrm{MWO}_{4}\right.$. $n \mathrm{H}_{2} \mathrm{O}, \mathrm{M}$ as labelled).

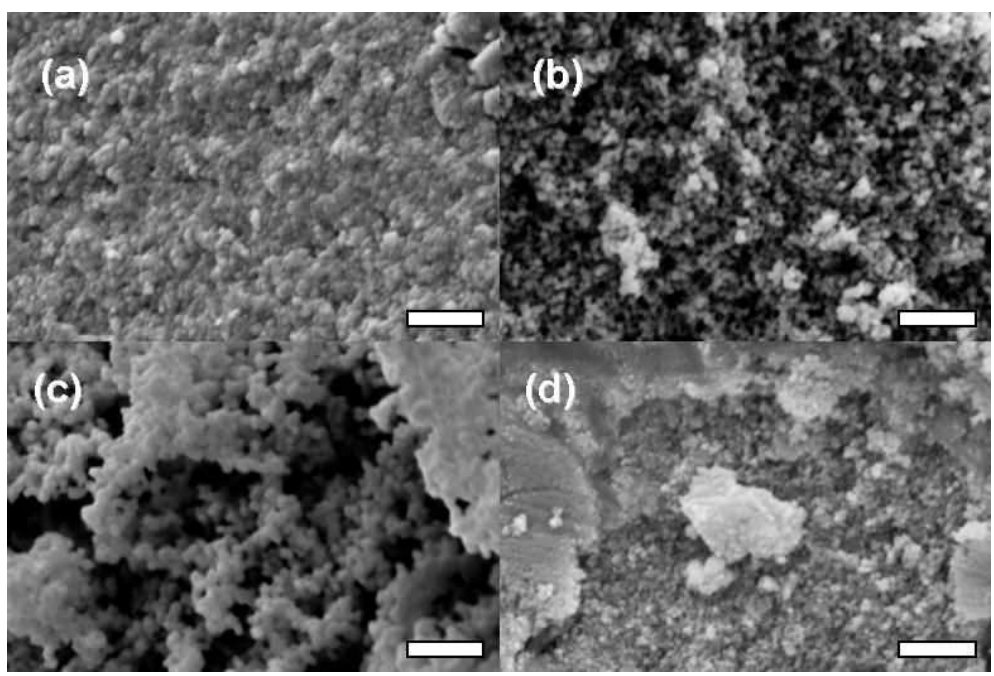

Figure 5.3: SEM micrographs of transition metal tungstate hydrates, $\mathrm{MWO}_{4}$. $n \mathrm{H}_{2} \mathrm{O}, \mathrm{M}=$ (a) Fe; (b) $\mathrm{Co}$; (c) Ni; (d) Cu. The scale bar corresponds to 2 $\mu \mathrm{m}$ in each case. 


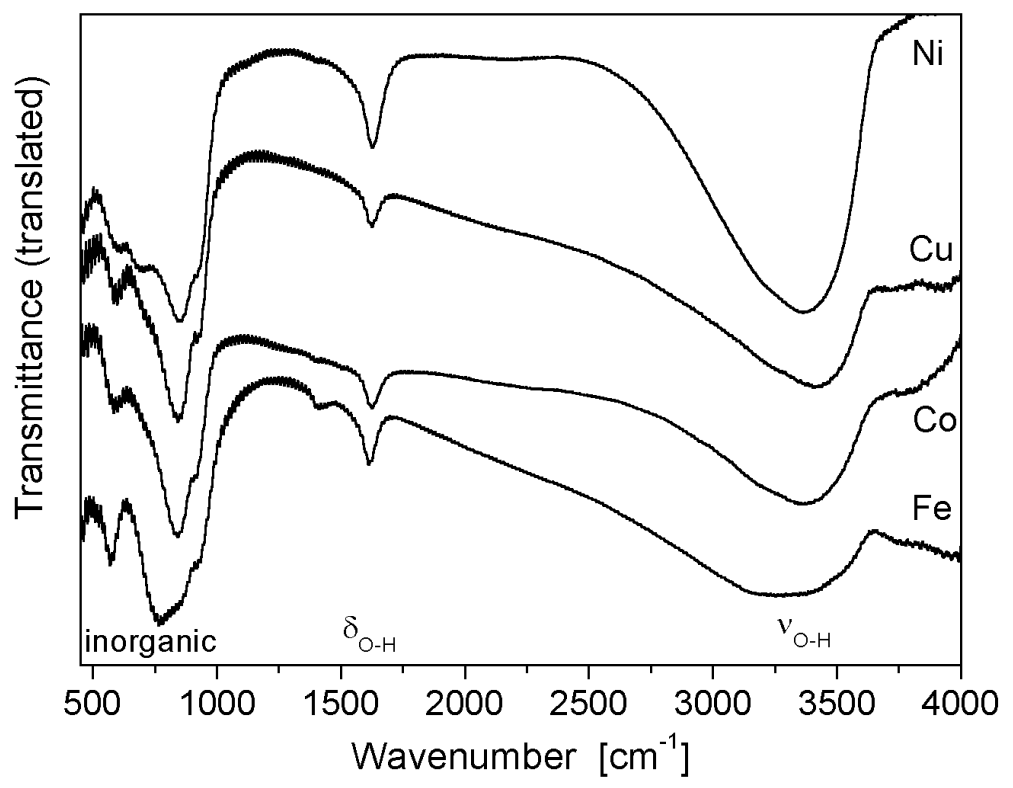

Figure 5.4: Infrared spectra of transition metal tungstate hydrates, $\mathrm{MWO}_{4}$. $n \mathrm{H}_{2} \mathrm{O}, \mathrm{M}$ as labelled.

\subsubsection{Comparison of methods}

Table 5.1 lists the various samples that were produced using each of the three methods.

The XRD patterns in Figure 5.1 compared the solution and melt methods for $(\mathrm{Mn}, \mathrm{W})$-DA6, illustrating that the same product had been formed. However, it is clear from the improved signal-to-noise ratio, narrower peaks and hence cleaner features of the solution-produced sample that this product is more crystalline than that produced using the melt method.

The improved crystallinity is also evident in SEM micrographs of the samples, as shown in Figure 5.5. In this figure, two (Mn,W)-DA6 samples are compared, one via the solution-based method and the other by the melt method. The scale is the same in both micrographs. While the solutionproduced sample consists of exfoliated platelets of uniform size up to 10 $\mu \mathrm{m}$ across, the sample produced via the melt method appears to have much smaller needlelike crystallites.

Similarly, a comparison was made between the melt and chimie douce methods. Figure 5.5(c) and (d) compare a (Co,W)-DA2 sample formed by 


\begin{tabular}{|c|c|c|c|}
\hline Hybrid material & Solution & Melt & Chimie douce \\
\hline \hline (Mn,W)-DA2 & $\sqrt{ }$ & - & - \\
(Mn,W)-DA6 & $\sqrt{ }$ & $\sqrt{ }$ & - \\
(Mn,W)-DA8 & $\sqrt{ }$ & - & - \\
(Mn,W)-DA12 & $\sqrt{ }$ & - & - \\
(Mn,W)-MA2 & $\times$ & - & - \\
(Mn,W)-MA6 & $\times$ & - & - \\
(Mn,W)-MA12 & $\sqrt{ }$ & - & - \\
(Mn,W)-phen & $\sqrt{ }$ & - & - \\
(Mn,W)-DAphen & $\sqrt{ }$ & - & - \\
\hline (Fe,W)-DA2 & $\sqrt{ }$ & - & - \\
(Fe,W)-DA6 & $\sqrt{ }$ & $\sqrt{ }$ & - \\
(Fe,W)-DA12 & $\sqrt{ }$ & - & - \\
(Fe,W)-DAphen & $\times$ & - & - \\
\hline (Co,W)-DA2 & $\sqrt{ }$ & $\sqrt{ }$ & $\sqrt{ }$ \\
(Co,W)-DA4 & - & $\times$ & - \\
(Co,W)-DA6 & $\times$ & $\sqrt{ }$ & $\sqrt{ }$ \\
(Co,W)-DA12 & - & - & $\times$ \\
(Co,W)-MA6 & - & - & $\sqrt{ }$ \\
\hline (Ni,W)-DA2 & - & - & $\sqrt{ }$ \\
(Ni,W)-DA6 & $\times$ & $\sqrt{ }$ & $\sqrt{ }$ \\
(Ni,W)-phen & - & $\sqrt{ }$ & - \\
(Ni,W)-DAphen & - & $\times$ & - \\
\hline (Cu,W)-DA2 & $\sqrt{ }$ & $\sqrt{ }$ & $\sqrt{ }$ \\
(Cu,W)-DA6 & $\times$ & $\sqrt{ }$ & - \\
(Cu,W)-phen & - & $\sqrt{ }$ & - \\
(Cu,W)-DAphen & - & $\times$ & - \\
\hline
\end{tabular}

Table 5.1: Synthesis methods for transition metal tungstate hybrids. $\sqrt{ }=$ successful, $\times=$ unsuccessful, $-=$ not attempted. ${ }^{1}$ from aqueous $\mathrm{Na}_{2} \mathrm{WO}_{4}$ solution. 


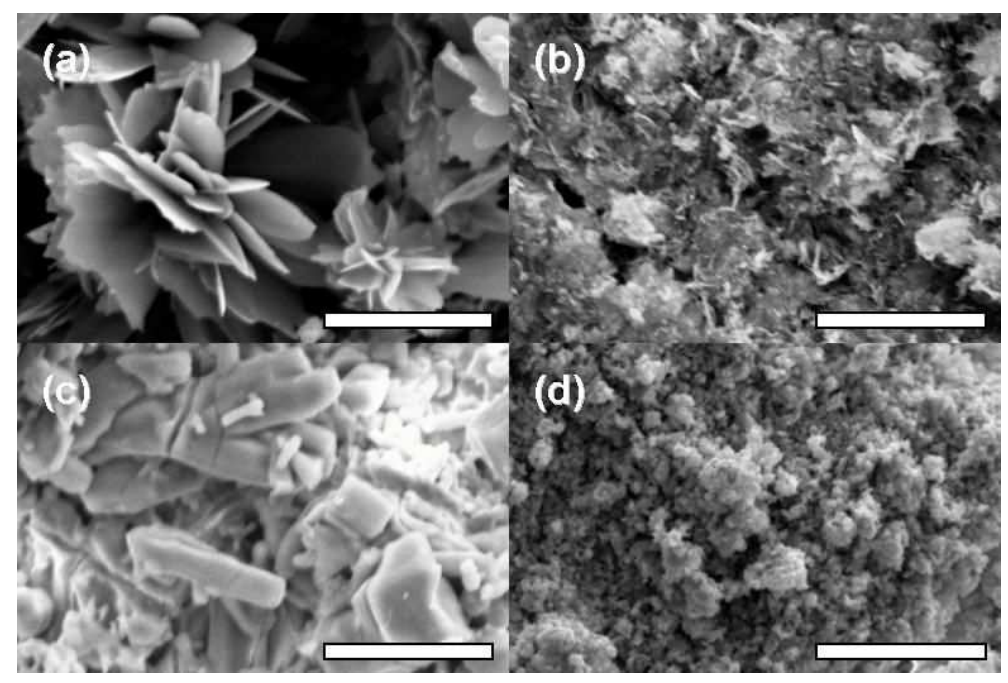

Figure 5.5: SEM micrographs of transition metal hybrids: (Mn,W)-DA6 via (a) solution and (b) melt methods, and (Co,W)-DA2 via (c) melt and (d) chimie douce methods. The scale bars correspond to $10 \mu \mathrm{m}$ in each case.

both of these methods. Blocky crystallites are formed using the melt method which are approximately ten times larger than those formed via a chimie douce reaction. The morphology of the starting $\mathrm{MWO}_{4} \cdot n \mathrm{H}_{2} \mathrm{O}$ material is probably responsible for these results, and indeed the general morphology of the chimie douce sample looks quite similar to that of the hydrate material (Figure 5.3). As the chimie douce reaction is performed at room temperature, the mobility of the inorganic component is not likely to be high enough to allow recrystallisation of the hybrid material as is normally evident in the solution-based method. Some dissolution of the inorganic hydrate material in melted amines is observed and the subsequent recrystallisation may result in the formation of larger crystallites, particularly if the hybrid structure is quite stable. This appears to be the case particularly for (Co,W)-DA2 when one compares the XRD of the same two samples, shown in Figure 5.6. The Bragg lines in the melt-produced (Co,W)-DA2 sample are much more pronounced than those of the chimie douce. Also shown in this figure are two $(\mathrm{Cu}, \mathrm{W})-\mathrm{DA} 2$ samples produced via the same two methods. In this case a number of peaks are stronger in the chimie douce sample than the melt sample, however there are more peaks in the melt sample and on average 


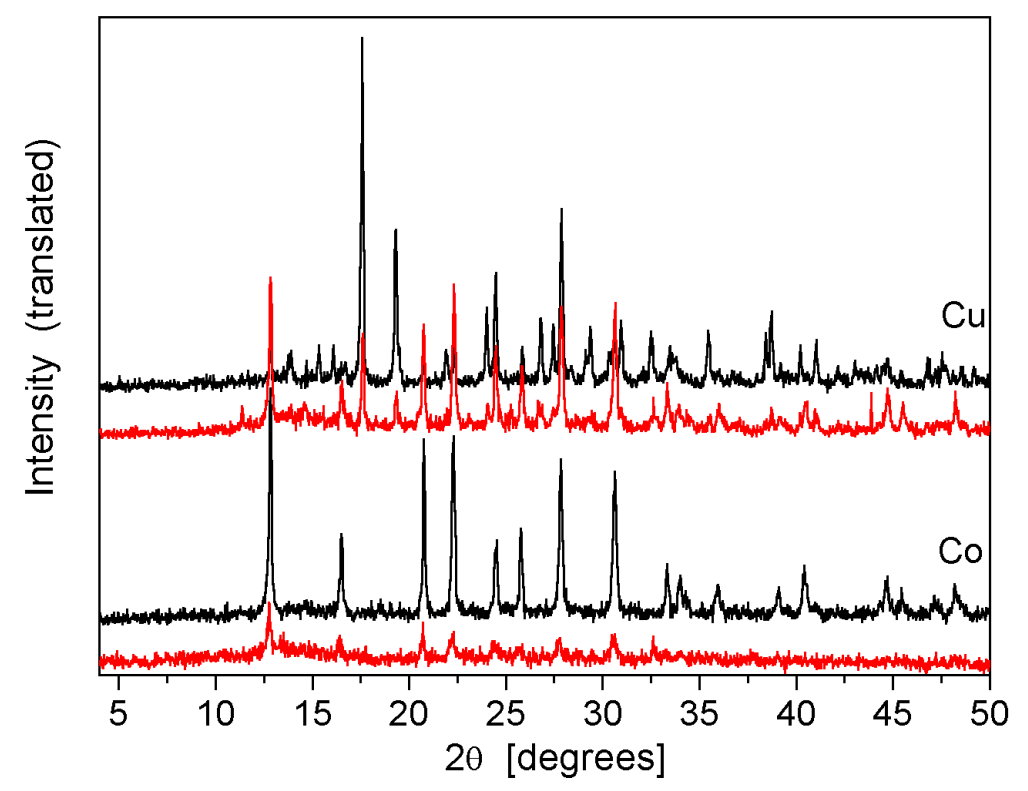

Figure 5.6: XRD patterns of two samples, $(\mathrm{Co}, \mathrm{W})-\mathrm{DA} 2$ and $(\mathrm{Cu}, \mathrm{W})-\mathrm{DA} 2$ (as labelled), synthesised by the melt, and chimie douce methods. Black line $=$ melt, red line $=$ chimie douce.

the signal-to-noise ratio is better. (The structure of both samples will be discussed later in Section 5.2.1.)

In general then, the crystallinity of samples produced via the three methods decreases from solution to melt to chimie douce. There are also limitations on whether each method can be used. As mentioned earlier, only Mn and Fe hybrids can be formed using the solution-based method due to the preferential formation of ammonium complexes of cobalt, nickel and copper in the $\mathrm{H}_{2} \mathrm{WO}_{4}$ /aqueous ammonia solution. The melt method can only be applied with organics that will melt cleanly in a particular temperature range $\left(-50\right.$ to $\left.200{ }^{\circ} \mathrm{C}\right)$ without reacting with air. The chimie douce method works best for small intercalated organics. Sometimes multiple intercalative steps are required to successfully intercalate a larger organic molecule, for instance by using a smaller organic intercalate as a preliminary step [175].

We now discuss structural and spectroscopic studies performed on the transition metal tungstate hybrids, following the same procedures as for the 
tungsten oxide-only hybrids.

\section{$5.2 \quad$ Results}

As was the case for the tungsten oxide hybrids, a number of characterisation techniques were used to determine the basic structural properties of the materials, including XRD, elemental analysis, electron microscopy, electron diffraction, IR and Raman spectroscopy, X-ray absorption spectroscopy, and optical spectroscopy.

\subsubsection{X-ray diffraction}

$\mathrm{XRD}$ is the primary method of initial characterisation of the hybrid materials. Because most of the transition metal hybrids, in particular those including $\mathrm{Co}, \mathrm{Ni}$ and $\mathrm{Cu}$ ions, are synthesised via methods that do not allow large crystallites to form, this affects the XRD patterns. In many samples the signal is too low to use for structural characterisation (indexing of XRD powder patterns and subsequent Rietveld refinement), other than to observe that a layered structure is present. Layered structures are characterised in XRD by a prominent series of $00 \ell$ lines (see [51, 261, 175] for examples of layered structures of $\mathrm{Ti}_{4} \mathrm{O}_{9}$, Aurivillius structure, and birnessite with organic intercalates which form fairly disordered hybrid phases displaying $00 \ell$ line series). XRD patterns of some examples of these poorly crystalline hybrid materials including transition metal ions are shown in Figure 5.7. In each case the $00 \ell$ lines are indicated, and the $d$-spacing calculated from the average of these. Additional peaks correspond to reflections whose indices include some $a$ and/or $b$ component. The (Mn,W)-MA12 sample shown in this graph represents the longest interlayer spacing achieved in this particular system to date. The other two samples, $(\mathrm{Ni}, \mathrm{W})-\mathrm{MA} 6$ and $(\mathrm{Cu}, \mathrm{W})$-phen also represent long interlayer spacings with relatively good signal-to-noise ratios.

XRD patterns are given for the (Mn,W)-DAn series in Figure 5.8a. Again, the $00 \ell$ lines are indicated and the corresponding $d$-spacings are plotted in Figure $5.8 \mathrm{~b}$ and compared with those of the W-DAn series from Chapter 3. 


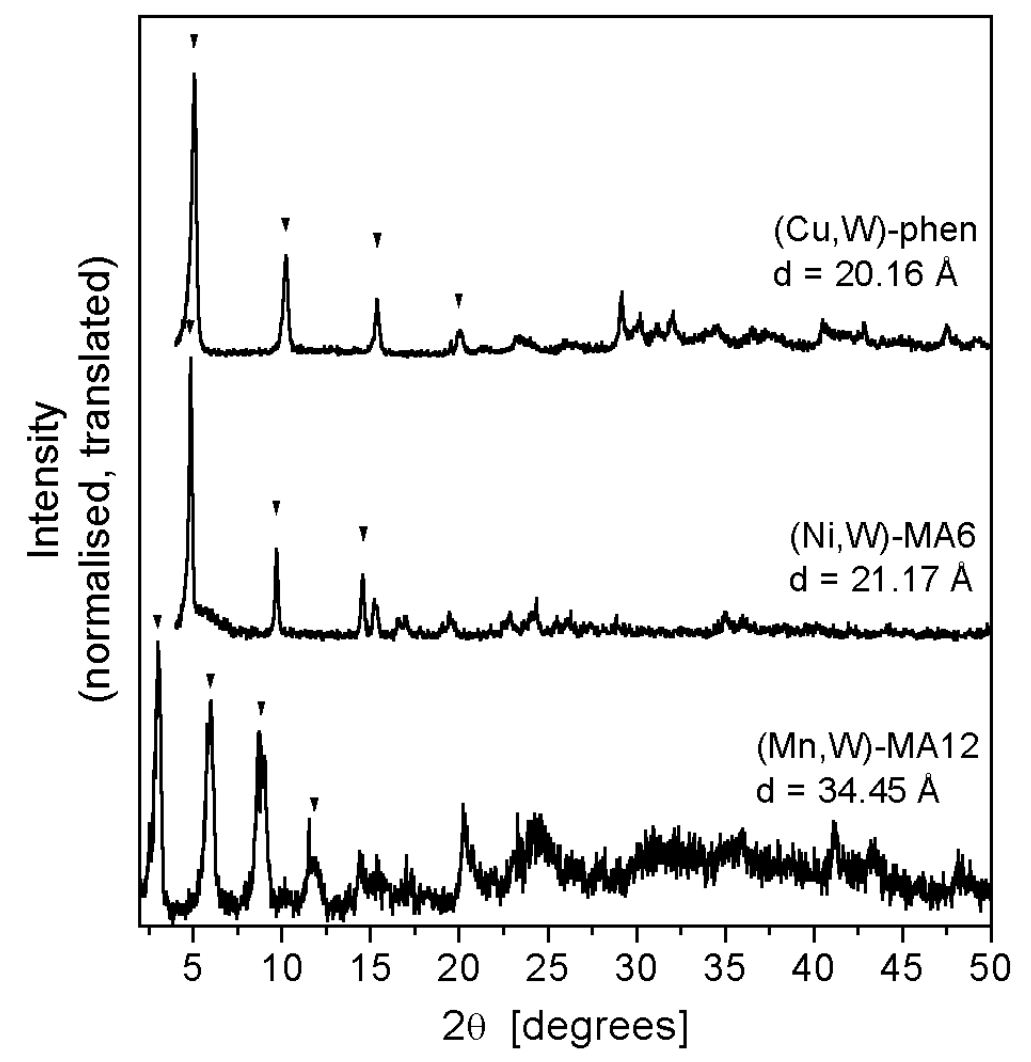

Figure 5.7: XRD patterns of three samples, as labelled, showing the series of $00 \ell$ lines characteristic of layered materials. 

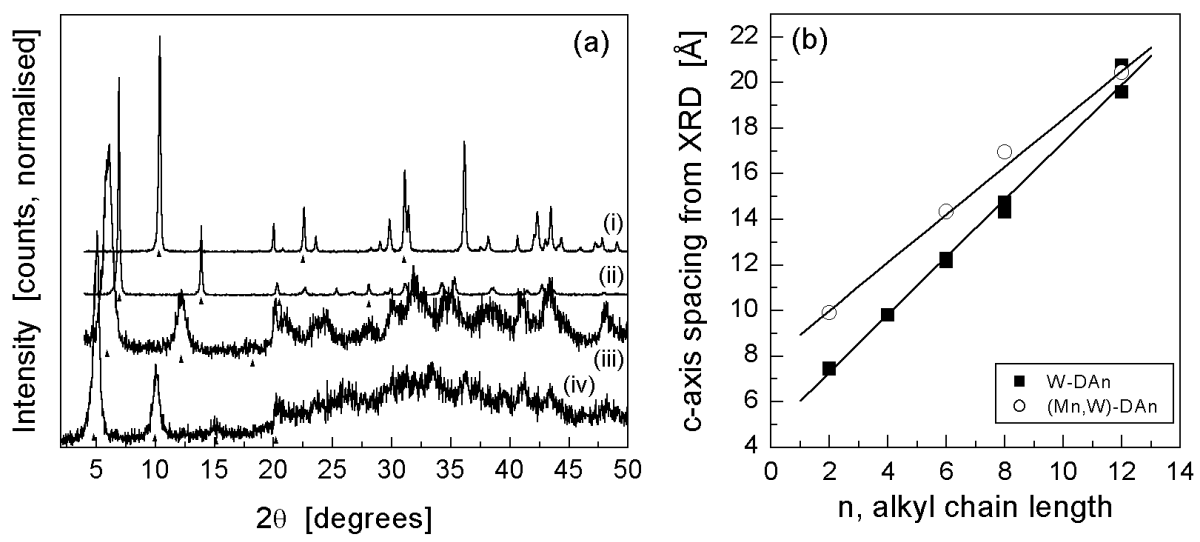

Figure 5.8: (a) XRD patterns of (Mn,W)-DAn hybrids, (i) $\mathrm{n}=2$, (ii) $\mathrm{n}=6$, (iii) $\mathrm{n}=8$, (iv) $\mathrm{n}=12$. The $00 \ell$ lines are indicated. (b) Plot of $d$-spacing obtained from the $00 \ell$ line spacing versus alkyl chain length of the organic intercalate, compared with the same results for the W-DAn series.

We can apply the same analysis of the slope and gradient of the best fit line as we did in Section 3.2.1.

The equation of the line is $d=1.051 n+7.893$. The lengthwise component of a $\mathrm{C}-\mathrm{C}$ bond in an alkane chain is $1.26 \AA$ per carbon atom (Section 3.2.1). As the gradient of the line is $1.051 \AA$ per carbon, this means the organic molecule is tilted at an angle $\theta$ of $\sin ^{-1}(1.051 / 1.026)=56.5^{\circ}$ to the inorganic layer, as indicated in Figure 5.9.

The inorganic layer spacing is calculated by

$$
d=d_{\text {oxide }}+2 d_{\Delta N}+\left((n-1) d_{C-C}+2 d_{N-C}\right) \sin \left(56.5^{\circ}\right)
$$

as shown in Figure 5.9. Thus when $n \rightarrow 0$, the expression becomes

$$
d=d_{\text {oxide }}+2 d_{\Delta N}+\left(2 d_{N-C}-d_{C-C}\right) \sin \left(56.5^{\circ}\right)
$$

Substituting $d=7.893, d_{N-C}=1.20 \AA$ and $d_{C-C}=1.26 \AA$, we obtain

$$
d_{\text {oxide }}+2 d_{\Delta N}=6.942 \stackrel{\circ}{\text {. }}
$$

This is almost exactly double the value of $3.56 \AA$ obtained for the tungsten oxide-only system in Section 3.2.1. It is not surprising that the oxide layer thickness has increased, as there are now two metal ions present per formula 


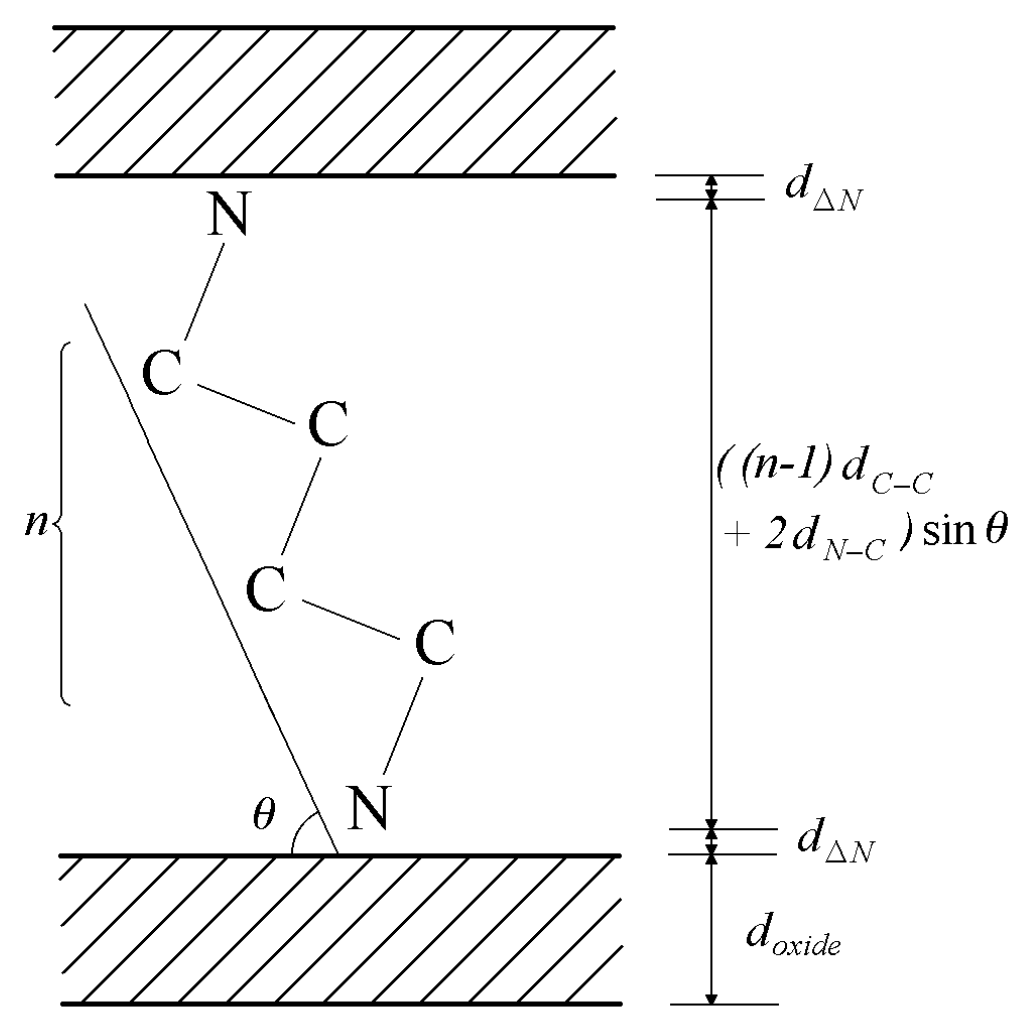

Figure 5.9: Diagram of (Mn,W)-DAn structure. 
unit, $\mathrm{MnWO}_{4}$. DAn (as evidenced by the microanalysis discussed in Section $5.2 .3)$.

Figure 5.10 shows the XRD pattern of $(\mathrm{Mn}, \mathrm{W})$-DAphen. While the pattern contains broad lines, it correlates to a layered structure of $11.18 \AA$. What is unusual about this sample is that the organic molecule, phenylenediamine (1,4-diaminobenzene), was able to be intercalated. As mentioned in Section 3.2 , organic molecules with the amino group directly attached to a benzene ring are unable to be used to form tungsten oxide hybrids presumably because of the steric hindrance of the benzene with the apical oxygen atoms. Not only does there appear to be less steric hindrance when manganese is incorporated into the structure, but the additional factor of DAphen being a bidentate molecule does not appear to adversely affect the stability of the hybrid. This contrasts with the tungsten oxide hybrids, where this particular intercalate is unstable. Therefore it appears that the inorganic layer structure of the manganese tungstate hybrids is different from that of the tungsten oxide hybrids, and/or the disposition of the organic intercalant is different. Either way, it is likely to have a more open structure in some respect to allow the incorporation of the bulky DAphen molecule. (This is explored further in Section 5.2.5.)

As indicated in Table 5.1, the only transition metal incorporated for which a substantial series of (DAn) hybrids were synthesised so far has been manganese. Manganese was the first transition metal ion to be incorporated in the tungsten oxide hybrid system. Also, the inability of the other metal ions to form hybrids via the standard solution method restricts the scope of organic molecules that can be included.

As mentioned in Section 5.1.1, (Co,W)-DA2, (Ni,W)-DA2 and $(\mathrm{Cu}, \mathrm{W})$ DA2 have nearly identical structures as indicated by XRD (shown in Figure 5.11). The copper sample displays several extra peaks that do not appear in the other two samples, which are most likely due to symmetry breaking due to copper ions often displaying several non-equivalent bond lengths, for 


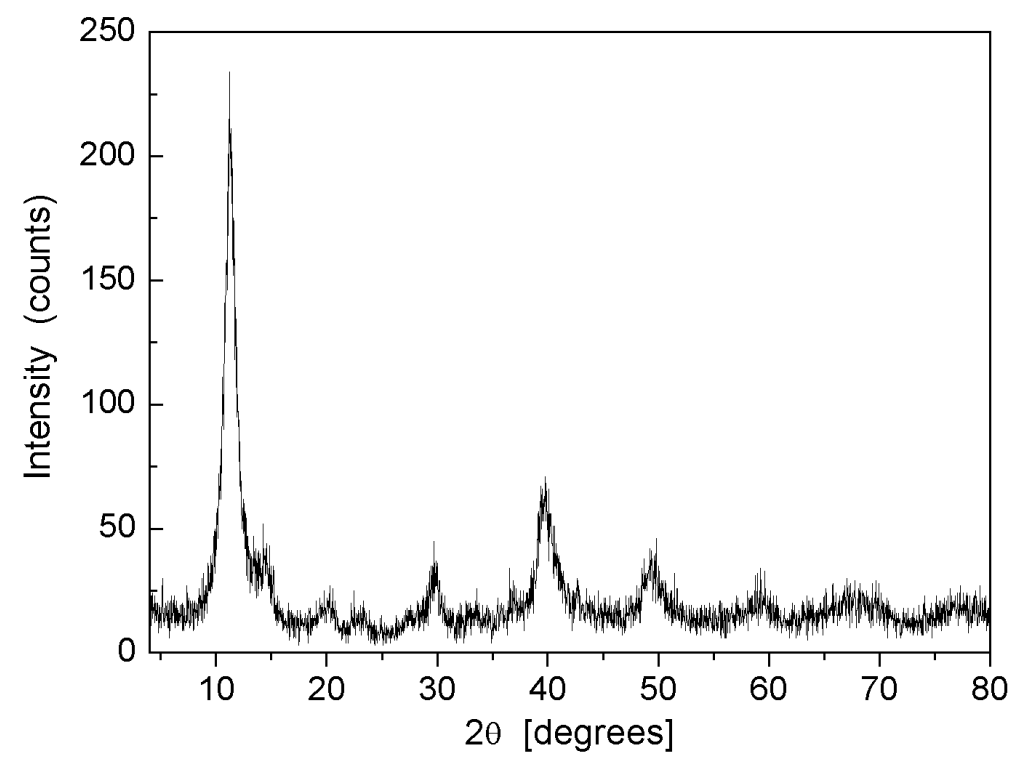

Figure 5.10: XRD pattern of (Mn,W)-DAphen.

example, due to the Jahn-Teller effect [111, 123]. More significantly, the lack of a distinct series of $00 \ell$ lines indicates that the structure formed is possibly non-layered.

Figure 5.12 shows the XRD patterns of the (M,W)-DAn samples, varying the transition metal ion $\mathrm{M}$ and comparing these with the 'pure' tungsten oxide hybrid W-DA6. The $00 \ell$ lines are indicated in each case. The patterns appear to fall into two distinct groups based on the $d$-spacing indicated by the $00 \ell$ lines: tungsten only, nickel and copper with a spacing of $12.2 \AA$, and manganese and cobalt with a spacing of $14.5 \AA$. While many of the other lines appear to be different, no inferences can be made about the structure within the inorganic layers from this data alone.

\subsubsection{Electron microscopy}

SEM images showing the microstructure of several different transition metal hybrids are given in Figure 5.13. Three (Mn,W)-DAn samples are shown in (a), (b) and (c); and three (M,W)-DA6 samples produced by the melt method are shown in figures (d), (e) and (f).

In Figure 5.13a and b we see the distinct platy morphology of the man- 


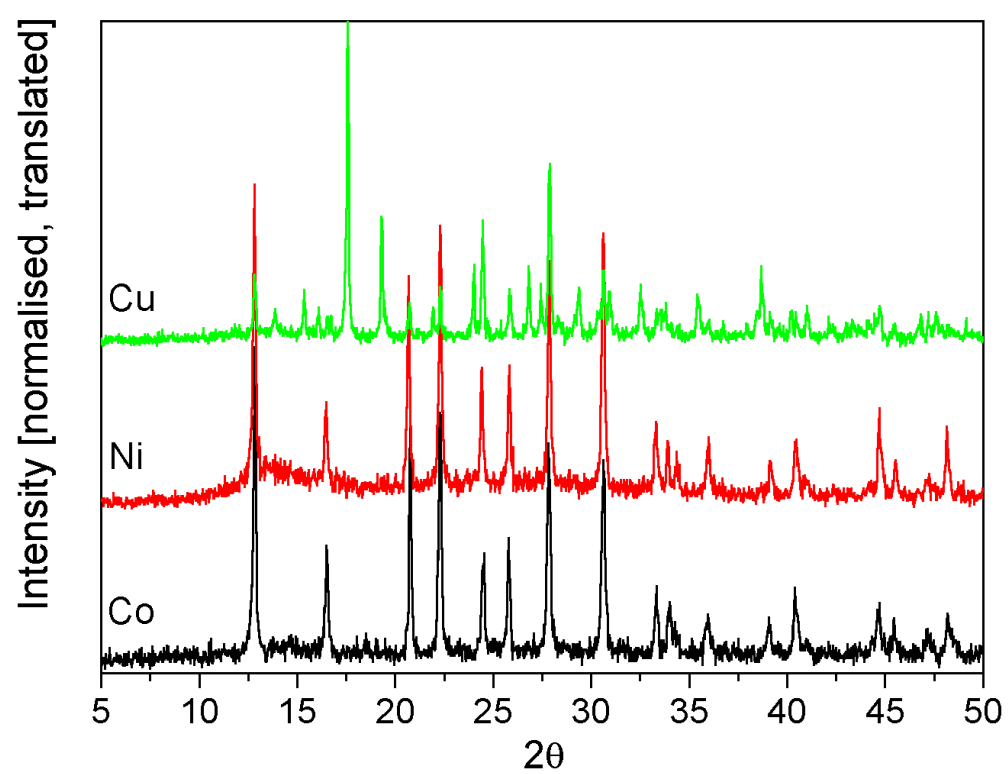

Figure 5.11: XRD patterns of transition metal hybrids (M,W)-DA2, M as labelled.

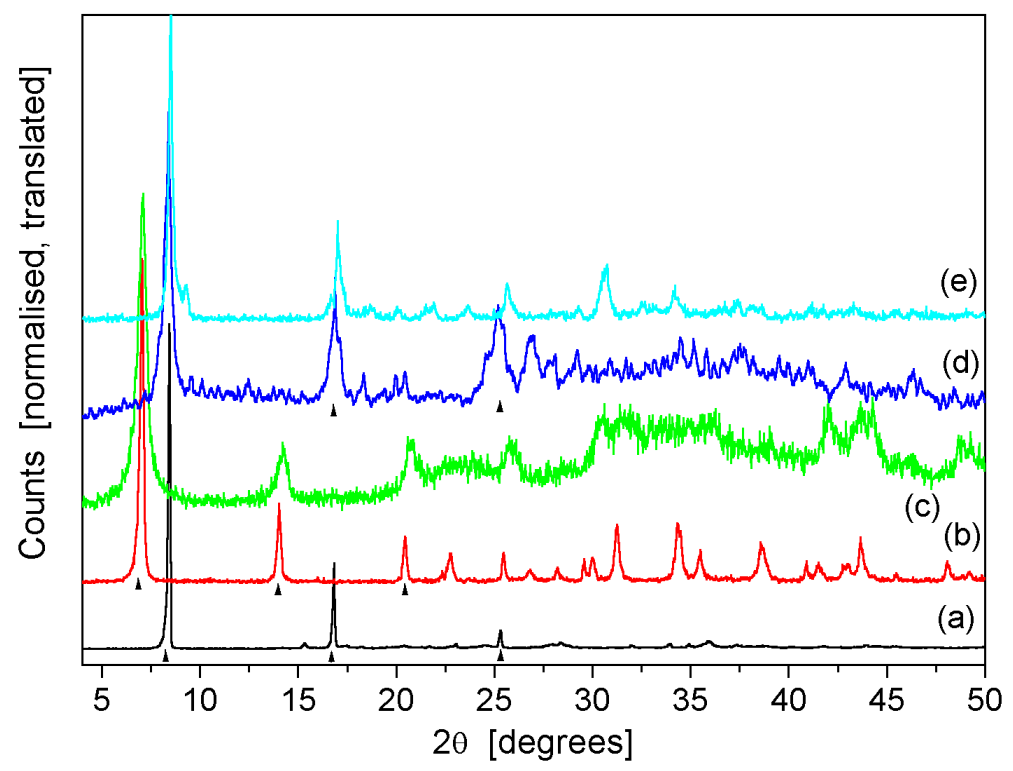

Figure 5.12: XRD patterns of transition metal hybrids (M,W)-DA6, M = (a) none; (b) Mn; (c) Co; (d) Ni; (e) Cu. 


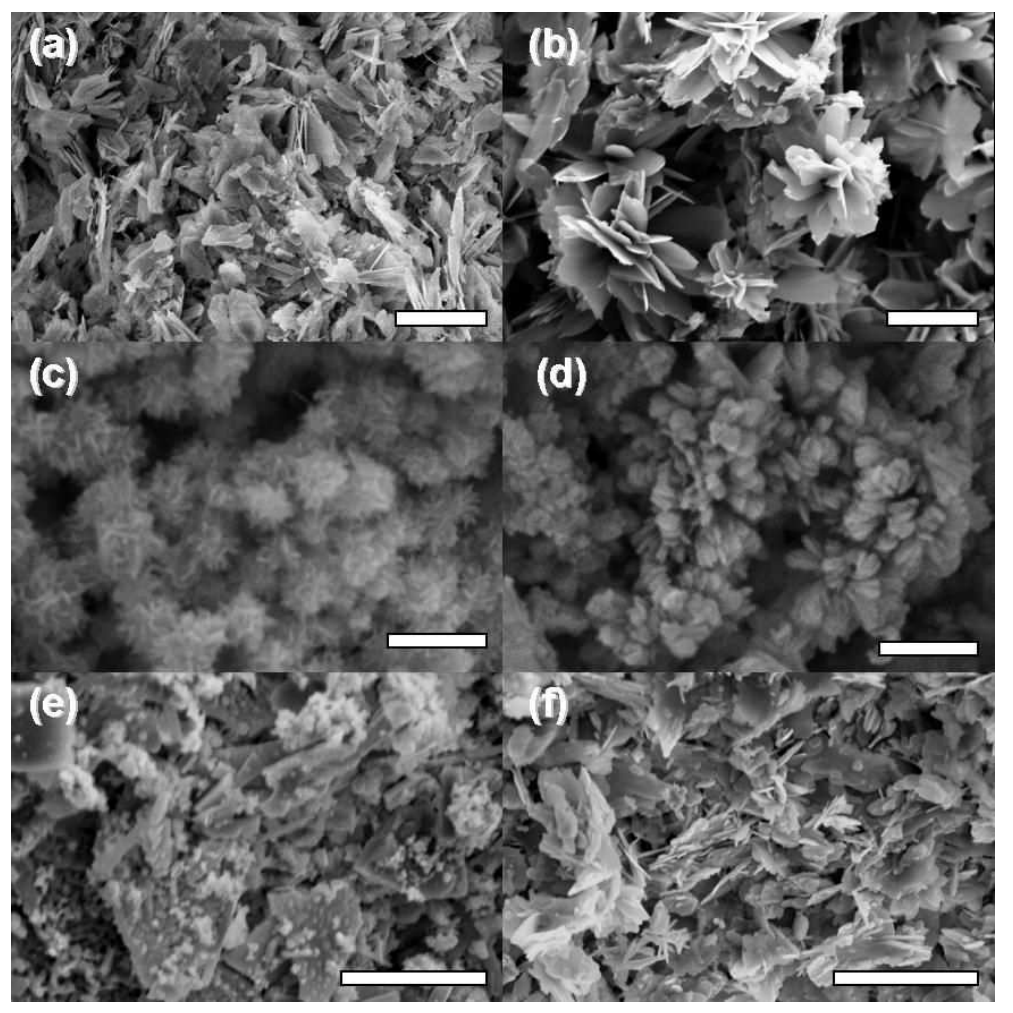

Figure 5.13: SEM micrographs of transition metal tungstate hybrids: (a) (Mn,W)-DA2; (b) (Mn,W)-DA6; (c) (Mn,W)-DA12; (d) (Co,W)-DA6; (e) (Ni,W)-DA6; (f) (Cu,W)-DA6. Scale bars correspond to $10 \mu \mathrm{m}$ for (a), (b), (e) and (f); and $2 \mu \mathrm{m}$ for (c) and (d). 
ganese hybrids which gives rise to the clear XRD patterns in Figure 5.8a. In Figure 5.13b in particular, the plates appear to be clumped together, like exfoliated spheres. At the centre of these 'clumps' it is conceivable that structures such as those shown in Figure $5.13 \mathrm{c}$ act as nucleation points. It is thought that due to the difficulty of forming the hybrids with long chains such as DA12, these nucleation centres were unable to form plates during the synthesis.

The (Co,W)-DA6 sample shown in Figure 5.13d also has the appearance of exfoliated spheres, although they are much smaller than those of the $(\mathrm{Mn}, \mathrm{W})-\mathrm{DA} 6$ sample in Figure 5.13b. (Ni,W)-DA6 and $(\mathrm{Cu}, \mathrm{W})-\mathrm{DA} 6$, in Figures 5.13e and f respectively, also demonstrate the formation of platelets of similar sizes to those of the manganese samples (up to $10 \mu \mathrm{m}$ across).

The platy morphology of the hybrids make them ideal for preparation for TEM studies.

A high-resolution TEM image of (Mn,W)-DA6 is shown in Figure 5.14. Indicated in this image are two axes evident from the arrangement of bright spots. These bright spots correspond to tungsten atoms, which have a high electron density (the atomic number of tungsten is 74). Comparing the spaces along each of the two axes, we observe that the spacing between tungsten atoms in one direction is almost exactly double that of the other direction. The manganese atoms are thought to lie in stripes between the tungsten atoms that are double-spaced. A glancing incidence view reveals the presence of several topological dislocations in this atomic ordering.

A SAED pattern of the same (Mn,W)-DA6 sample is shown in Figure 5.15. Evident in this diffraction pattern are two almost orthogonal axes, one showing a four-fold superlattice (and corresponding to a spacing of $10.42 \AA$ ) and the other showing a two-fold superlattice (with a spacing of $5.03 \AA$ ). As in Section 3.2.2, the uncertainty in the absolute values is $\pm 10 \%$ but the ratio of the two axes and the angle between them can be determined more accurately than this. The ratio is 2.070 and the angle is $89.7^{\circ}$, implying that the $a b$ plane is almost a square, doubled in one direction. 


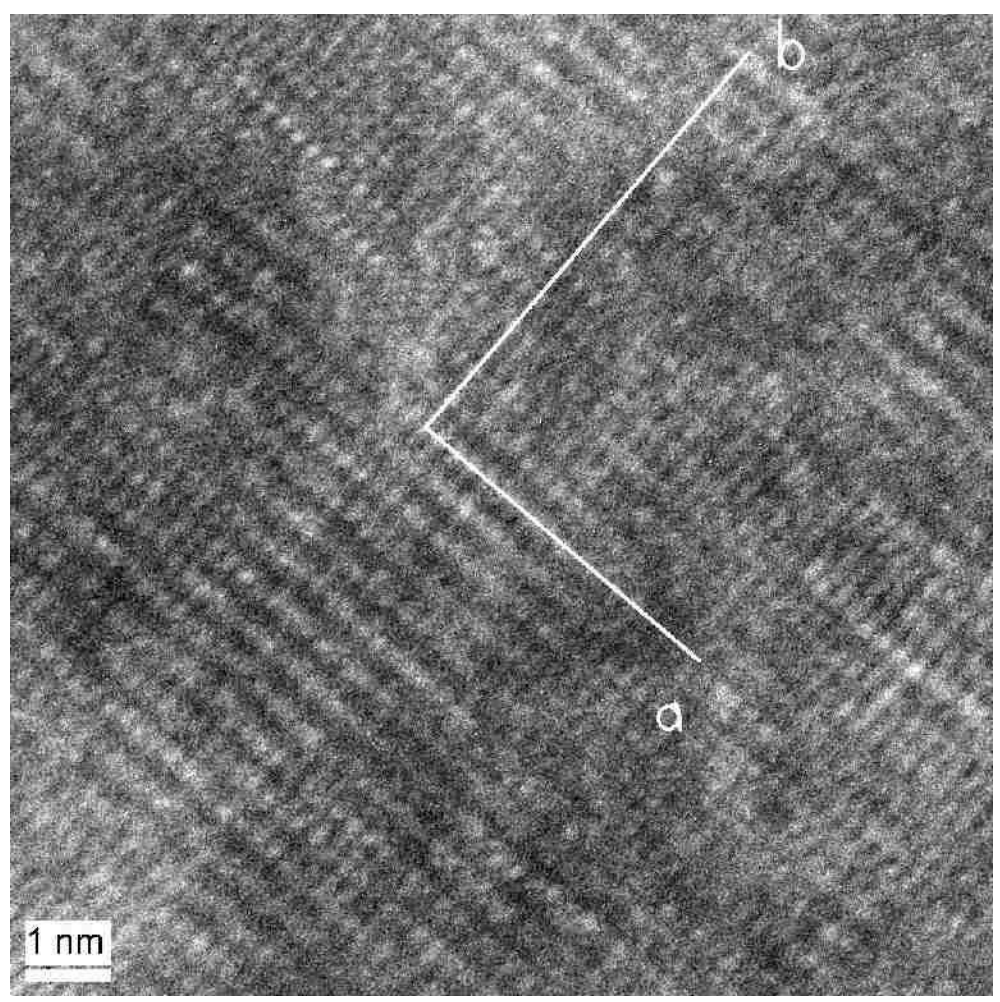

Figure 5.14: High-resolution TEM image of $(\mathrm{Mn}, \mathrm{W})-\mathrm{DA} 6$, with axes indicated. 


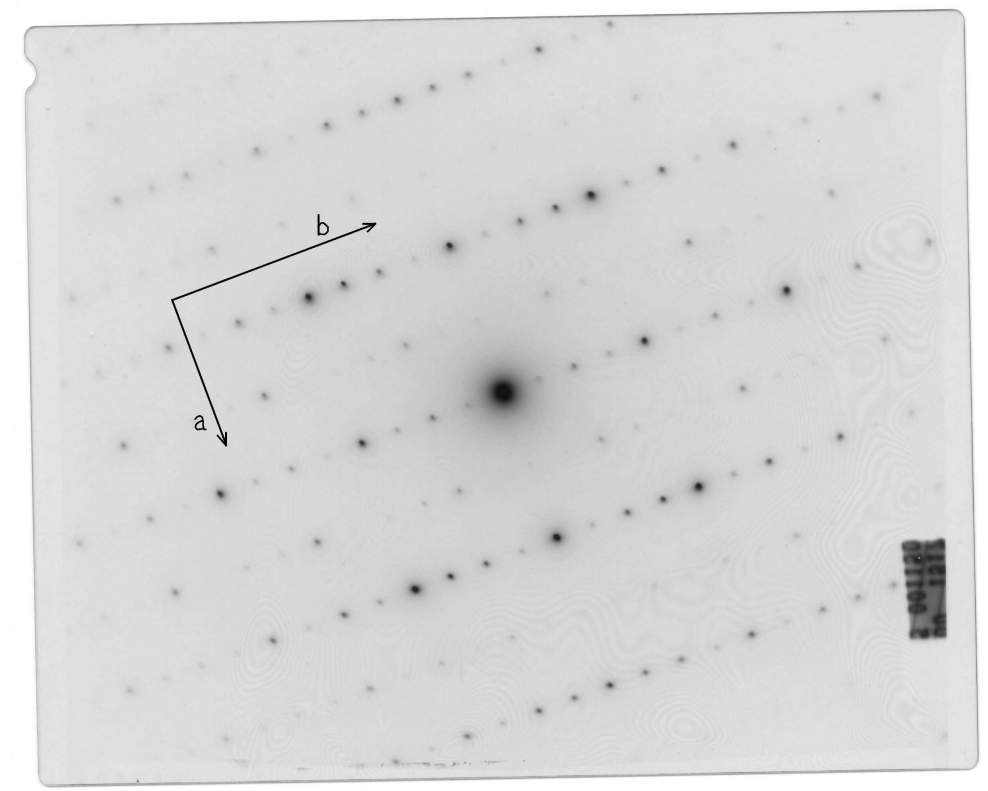

Figure 5.15: Electron diffraction image of $(\mathrm{Mn}, \mathrm{W})-\mathrm{DA} 6$, showing the $a b$ plane.

\subsubsection{Elemental microanalysis}

Many of these transition metal tungstate hybrids were subjected to $\mathrm{C}, \mathrm{H}$ and $\mathrm{N}$ elemental analysis like their tungsten oxide-only counterparts (Section 3.2.3). These samples were also examined using the EDX system incorporated in the SEM, to determine the metal-to-tungsten ratio. Further, it is known from XANES spectra (Section 5.2.5) that tungsten is present as $\mathrm{W}^{6+}$ and manganese as $\mathrm{Mn}^{2+}$. The results are tabulated in Table 5.2.

As can be seen from the table, the metal:tungsten:oxygen ratios are always very close to the inferred stoichiometric values of 1:1:4. For those samples synthesised from $\mathrm{MWO}_{4} \cdot n \mathrm{H}_{2} \mathrm{O}$ via the melt or chimie douce methods, this is not surprising. However for the manganese samples produced by the solution-based method, the stoichiometry indicated by EDX is independent over quite a wide range of the molar ratio of starting materials $\left(\mathrm{Mn}_{0.3} \mathrm{~W}_{0.7}-\right.$ $\left.\mathrm{Mn}_{0.7} \mathrm{~W}_{0.3}\right)$. For all samples, no other elements (e.g. from starting materials) were detected in the EDX spectra.

The organic ratios are also in good agreement with the expected values: 


\begin{tabular}{|c|ccc|ccc|c|}
\hline Hybrid material & \multicolumn{3}{|c}{ Inorganic } & \multicolumn{3}{c|}{ Organic } & I:O Ratio \\
& $\mathrm{M}$ & $\mathrm{W}$ & $\mathrm{O}$ & $\mathrm{C}$ & $\mathrm{H}$ & $\mathrm{N}$ & \\
\hline \hline (Mn,W)-DA2 & 1.059 & 0.941 & 3.883 & 2 & 10.44 & 1.84 & $1: 0.40$ \\
(Mn,W)-DA6 & 1.129 & 0.871 & 3.742 & 6 & 17.3 & 1.89 & $1: 0.39$ \\
(Mn,W)-DA8 & 1.226 & 0.774 & 3.549 & 8 & 19.41 & 1.85 & $1: 0.54$ \\
(Mn,W)-DA12 & 0.897 & 1.103 & 4.205 & 12 & 27.58 & 1.93 & $1: 0.53$ \\
\hline (Fe,W)-DA2 & 1.096 & 0.904 & 3.809 & 2 & 9.19 & 1.89 & $1: 1.12$ \\
(Fe,W)-DA6 & 0.828 & 1.172 & 4.354 & 6 & 20.67 & 1.94 & $1: 0.42$ \\
\hline (Co,W)-DA2 & 0.875 & 1.125 & 4.249 & 2 & 8.84 & 1.86 & $1: 0.72$ \\
(Co,W)-DA6 & 0.908 & 1.092 & 4.184 & 6 & 15.73 & 1.95 & $1: 0.71$ \\
\hline (Ni,W)-DA2 & 1.181 & 0.819 & 3.758 & 2 & 7.57 & 1.94 & $1: 2.77$ \\
(Ni,W)-DA6 & 0.957 & 1.043 & 4.267 & 6 & 18.35 & 2.19 & $1: 1.28$ \\
\hline (Cu,W)-DA2 & 0.954 & 1.046 & 4.092 & 2 & 9.46 & 1.87 & $1: 2.00$ \\
\hline
\end{tabular}

Table 5.2: Elemental analysis results for transition metal hybrids, showing the inorganic results calculated from EDX analysis, organic results calculated from elemental microanalysis, and the inorganic:organic (I:O) ratio. Calculated for $\mathrm{M}+\mathrm{W}=2, \mathrm{C}=$ number of carbons in chain.

DA2 $-\mathrm{C}_{2} \mathrm{H}_{8-10} \mathrm{~N}_{2}$

DA6 - $\mathrm{C}_{6} \mathrm{H}_{16-18} \mathrm{~N}_{2}$

DA $8-\mathrm{C}_{8} \mathrm{H}_{20-22} \mathrm{~N}_{2}$

DA12 - $\mathrm{C}_{12} \mathrm{H}_{28-30} \mathrm{~N}_{2}$

However, the inorganic:organic ratios are often quite different from the 1:1 seen in the tungsten oxide-only samples. In most cases the ratio is slightly less than 1:0.5, indicating an inorganic-rich phase is consistently formed. However for the nickel and copper samples the ratios are greater than 1:1, indicating an organic-rich phase has formed.

\subsubsection{IR and Raman spectroscopy}

Infrared and Raman spectra were obtained as for the tungsten oxide hybrids, and the spectra for the manganese tungstate hybrid series are shown in Figures 5.16 and 5.17.

The first point to notice in both the infrared and Raman spectra is how similar the spectra are for the hybrids. In the infrared spectra the labelled regions show the major organic vibrational modes: $\mathrm{N}-\mathrm{H}$ stretch at 3240-3340 


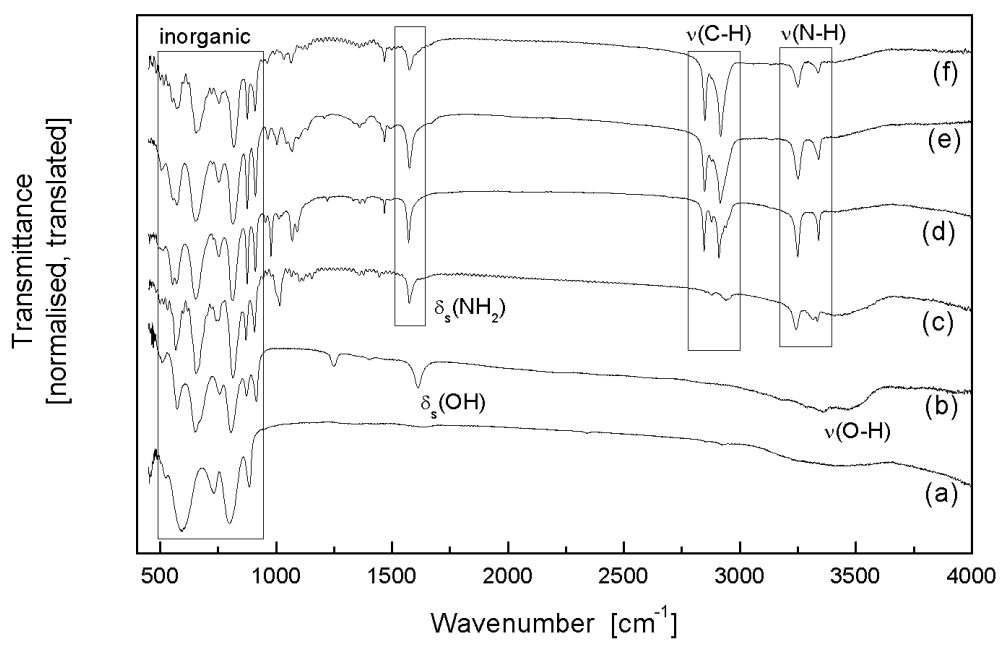

Figure 5.16: Infrared spectra of manganese tungstate-based materials: (a) $\mathrm{MnWO}_{4}$; (b) $\mathrm{MnWO}_{4} \cdot n \mathrm{H}_{2} \mathrm{O}$; (c) (Mn,W)-DA2; (d) (Mn,W)-DA6; (e) (Mn,W)-DA8; (f) (Mn,W)-DA12.

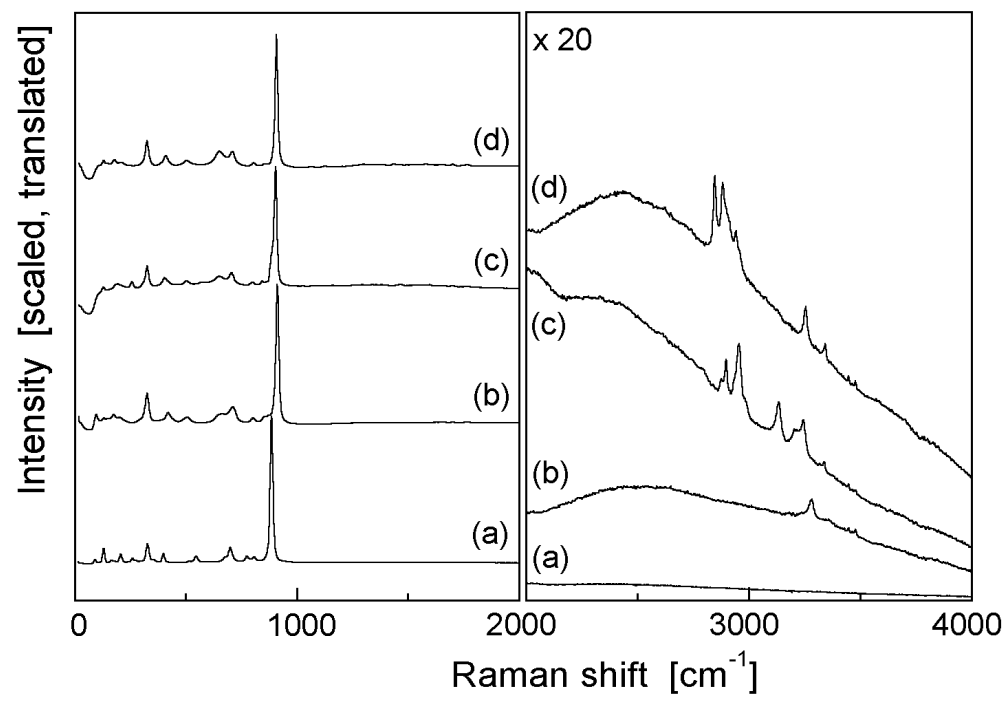

Figure 5.17: Raman spectra of manganese tungstate-based materials: (a) $\mathrm{MnWO}_{4}$; (b) $\mathrm{MnWO}_{4} \cdot n \mathrm{H}_{2} \mathrm{O}$; (c) (Mn,W)-DA2; (d) (Mn,W)-DA6. 
$\mathrm{cm}^{-1}$, C-H stretch 2840-2940 $\mathrm{cm}^{-1}, \mathrm{NH}_{2}$ bending $1570 \mathrm{~cm}^{-1}$. Comparing these with the spectra of the tungsten oxide hybrids earlier, the $\mathrm{N}-\mathrm{H}$ stretching lines are much sharper, there are only two $\mathrm{NH}_{2}$ bending modes, and there are no broad features near $2100 \mathrm{~cm}^{-1}$. These factors together indicate that the amine end-groups are neutral $-\mathrm{NH}_{2}$ terminations rather than charged $-\mathrm{NH}_{3}{ }^{+}$as in the tungstates. At the low-frequency end, the lines corresponding to inorganic vibrational modes are the same for the hybrids and the hydrate material. However they are different from the 'parent' compound, $\mathrm{MnWO}_{4}$, which is also shown for reference. These lines have been reported previously for the hydrate compound, although no structural information was given [151].

The Raman spectra (Figure 5.17) also appear identical for the hybrids and hydrate system (with the exception, of course, of the organic modes at high Raman shifts). Again, there are differences between these and the reference compound $\mathrm{MnWO}_{4}$. As in the tungsten oxide hybrids, a strong peak corresponding to $\mathrm{W}=\mathrm{O}$ bonding is evident near $900 \mathrm{~cm}^{-1}$. However, unlike the tungsten oxide hybrids, the frequency of this line increases from 886 $\mathrm{cm}^{-1}$ for $\mathrm{MnWO}_{4}$ to $906 \mathrm{~cm}^{-1}$ for the hydrates and hybrids. The structure of $\mathrm{MnWO}_{4}$ consists of an interlocking network of distorted $\mathrm{MnO}_{6}$ and $\mathrm{WO}_{6}$ octahedra which align in alternating planes of $\mathrm{Mn}$ and $\mathrm{W}$ cations [158]. It is therefore quite conceivable that when the layers form in the case of the hybrids and hydrate, some $\mathrm{W}=\mathrm{O}$ bonds are formed which are then shorter and stronger than those in $\mathrm{MnWO}_{4}$. The inorganic structures are identical for the hybrids and hydrates, and there is no direct interaction between the neutral organic or water molecules and the inorganic layer, as indicated from analysis of the infrared spectra.

As mentioned in Section 5.2.1 and indicated in Table 5.1, manganese is the only transition metal for which a number of different hybrids have been produced. While a select few of the other hybrids have been studied via IR and Raman spectroscopy, it is difficult to compare them because of the lack of data. None of the spectra show the characteristic peak at $2100 \mathrm{~cm}^{-1}$ corresponding to $-\mathrm{NH}_{3}{ }^{+} \ldots{ }^{-} \mathrm{O}$ hydrogen-bonding, indicating that like the man- 


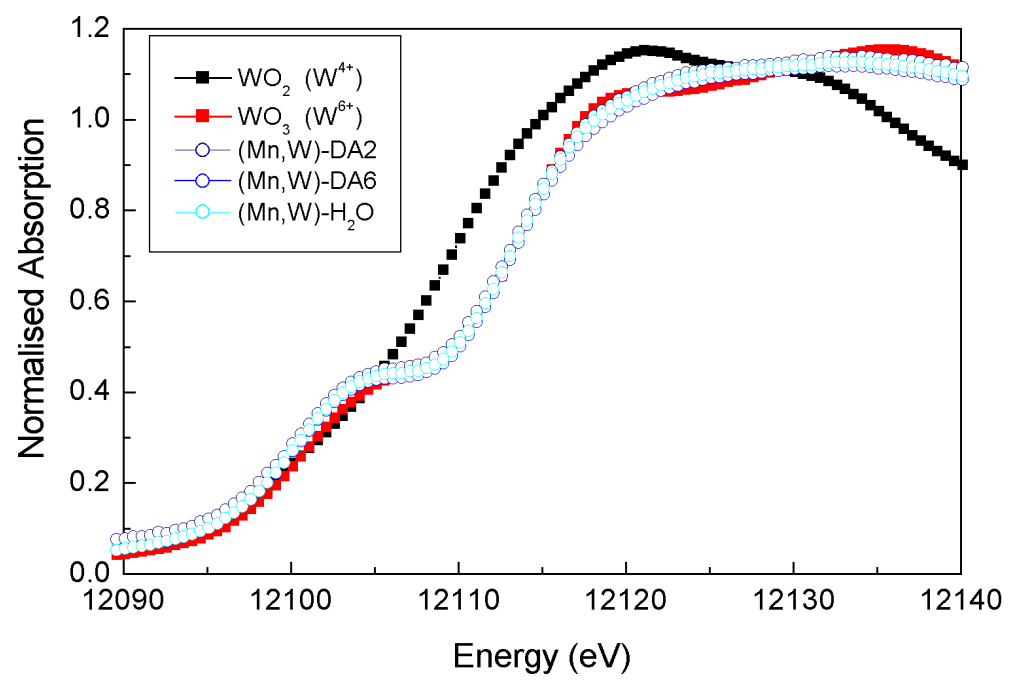

Figure 5.18: XANES spectra of (Mn,W)-DAn at the W L1-edge.

ganese system, these too are comprised of a neutrally-charged inorganic layer and neutral amine-terminated organic intercalate molecules. Other than this the spectra contain the expected organic and inorganic modes similar to those observed in the manganese series.

\subsubsection{XANES and EXAFS}

X-ray absoption near edge spectroscopy (XANES) and extended x-ray absorption fine structure (EXAFS) measurements were performed on $\mathrm{MnWO}_{4}$. $n \mathrm{H}_{2} \mathrm{O}$, (Mn,W)-DA2 and (Mn,W)-DA6, as described in Section 2.4.

XANES spectra are shown for the W L1-edge in Figure 5.18 and for the Mn K-edge in Figure 5.19. The position of the edge corresponds to the valence of the ion being probed. In the tungsten spectra, all three curves are extremely similar to the reference curve of $\mathrm{WO}_{3}$, indicating that the tungsten ions are present as $\mathrm{W}^{6+}$. In the manganese spectra, the curves are compared with three reference compounds, $\mathrm{MnO}, \mathrm{Mn}_{2} \mathrm{O}_{3}$ and $\mathrm{MnO}_{2}$ ( $\mathrm{Mn}$ valences of $+2,+3$ and +4 respectively). Once again, all three compounds are similar and correspond to a manganese valence of $\mathrm{Mn}^{2+}$. This serves to validate the EDX data, which gave stoichiometries consistent with $\mathrm{MnWO}_{4}$, a structure in which the metal ions are present as $\mathrm{Mn}^{2+}$ and $\mathrm{W}^{6+}$. 


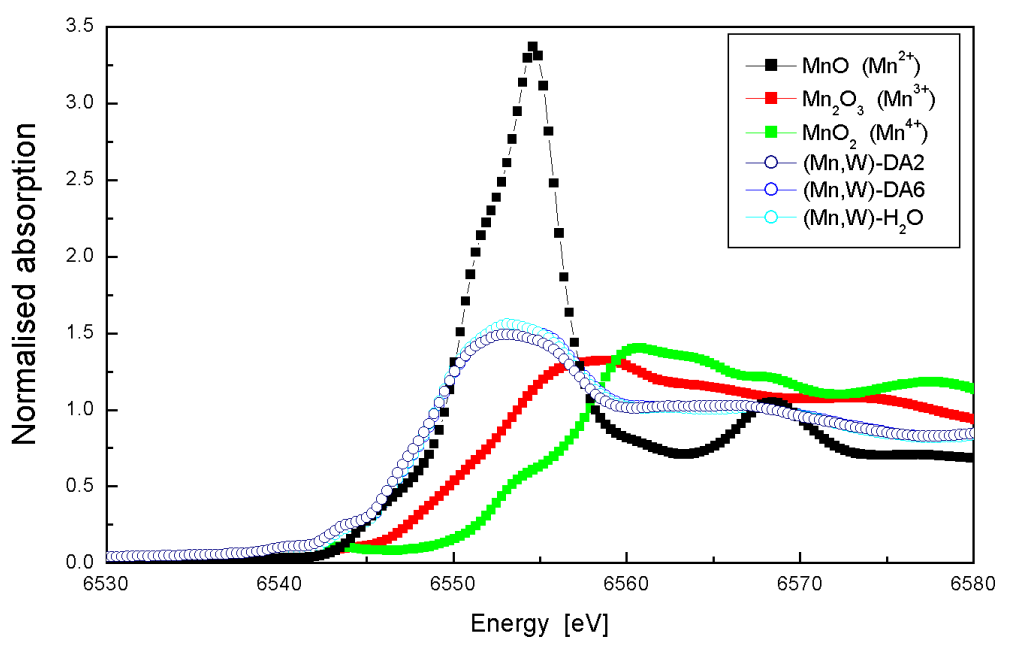

Figure 5.19: XANES spectra of (Mn,W)-DAn at the Mn K-edge.

\begin{tabular}{|c|c|c|}
\hline Shell & Length $(\AA)$ & Co-ordination number \\
\hline \hline W-O & $1.737 \pm 0.021$ & $2.11 \pm 0.64$ \\
W-O & $2.121 \pm 0.022$ & $4.82 \pm 1.39$ \\
W-W & $3.233 \pm 0.024$ & $6.63 \pm 3.58$ \\
W-O & $3.337 \pm 0.033$ & $12.21 \pm 3.61$ \\
\hline Mn-O & $2.124 \pm 0.007$ & $4.76 \pm 0.40$ \\
Mn-Mn & $3.409 \pm 0.041$ & $1.28 \pm 0.80$ \\
\hline
\end{tabular}

Table 5.3: Averaged EXAFS results from fitting analysis of manganese tungstate hybrids.

The EXAFS spectra for all three compounds are virtually identical. Good fits to the spectra were obtained by using the structure of the parent compound $\mathrm{MnWO}_{4}$ as a starting point. A plot of the EXAFS data in R-space with the best fits are shown in Figure 5.20 for the W L1-edge and Figure 5.21 for the Mn K-edge. The results are summarised in Table 5.3.

In the table, the co-ordination numbers are quite uncertain past the third $(\mathrm{W}-\mathrm{W})$ shell $^{1}$. This is most likely due to the presence of several shells in this region, to which fits were also attempted without success. $\mathrm{MnWO}_{4}$ gives the following shells: W-W $3.2813 \AA$, W-O $3.3594 \AA$, W-Mn $3.5214 \AA, \mathrm{W}-\mathrm{Mn}$ $3.5823 \AA, \mathrm{W}-\mathrm{O} 3.6018 \AA$. It is possible then that the $\mathrm{W}-\mathrm{W}$ and $\mathrm{W}-\mathrm{O}$ shells

\footnotetext{
'Shell' is the terminology used in EXAFS to refer to the positions of neighbouring atoms at increasing distances from the atom being probed.
} 


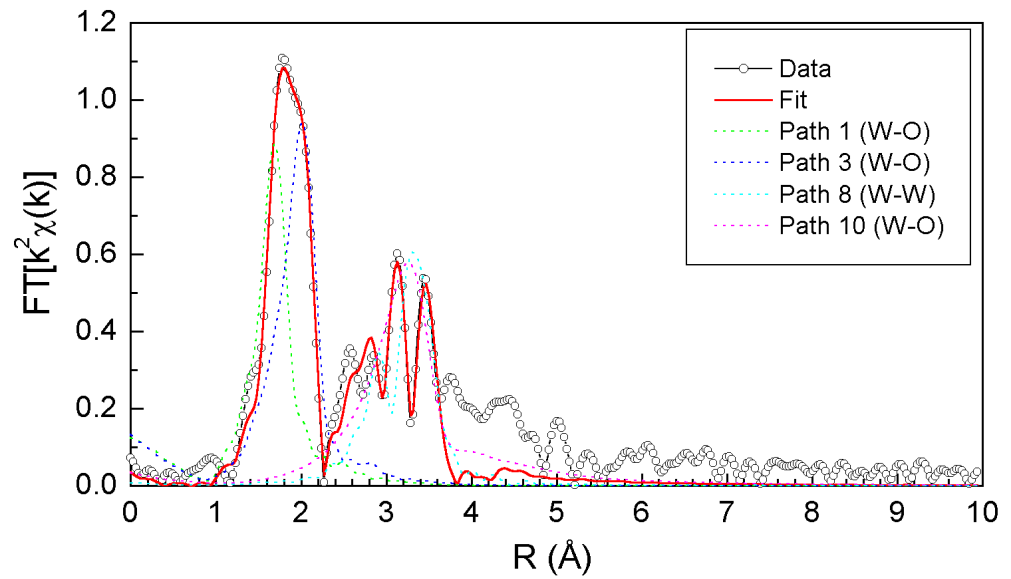

Figure 5.20: EXAFS spectra for (Mn,W)-DAn from the W L1-edge.

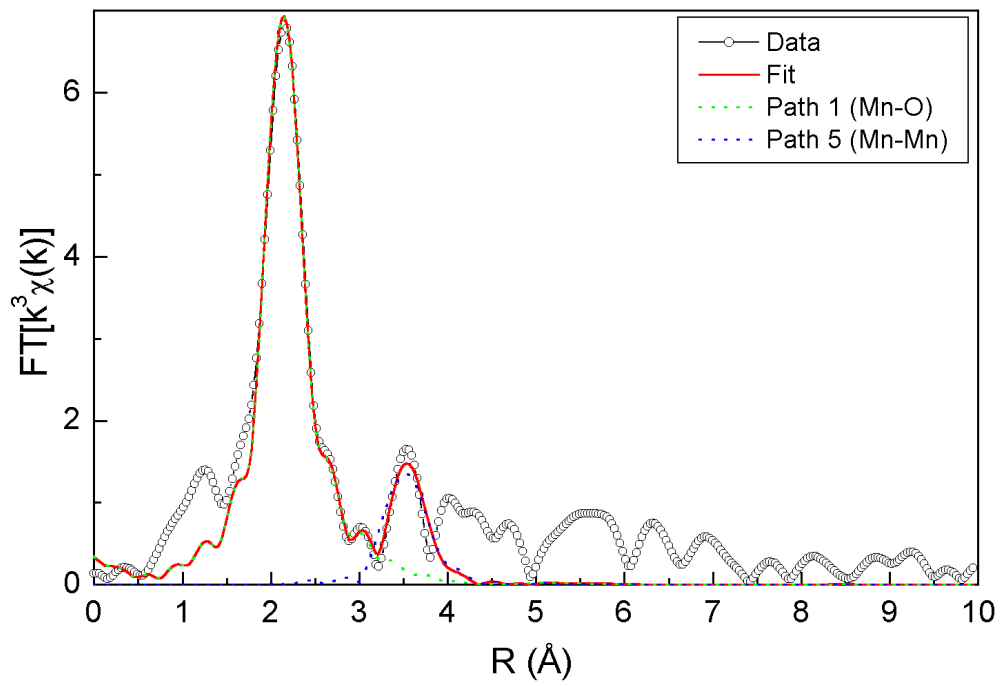

Figure 5.21: EXAFS spectra for (Mn,W)-DAn from the Mn K-edge. 
reported in the table have much higher co-ordination numbers than in the actual system, as the other $\mathrm{W}-\mathrm{Mn}$ and $\mathrm{W}-\mathrm{O}$ shells have not been included in the fit, but would contribute to the spectra in the same region. It is also important to remember that EXAFS gives an averaged result.

The results can be summarised as follows. 2 shorter $\mathrm{W}-\mathrm{O}$ bonds (1.7 $\AA$ ) and 4 longer ones $(2.1 \AA)$ are observed, which most likely correspond to the normally distorted $\mathrm{WO}_{6}$ octahedra. In $\mathrm{MnWO}_{4}$ the tungsten oxide octahedra are edge shared, and the $\mathrm{W}-\mathrm{W}$ distance is consistent with both in-plane longer $\mathrm{W}-\mathrm{O}$ bonds or if a bilayer was present, from the distance between adjacent tungsten atoms in the two layers. The next $\mathrm{W}-\mathrm{O}$ shell observed probably corresponds to the distance from one tungsten atom to the apical oxygen atoms of its neighbouring octahedra.

Fitting to the manganese data was more difficult, indicating that the positions of the manganese atoms are more disordered than those of the tungsten. In $\mathrm{MnWO}_{4}$ the $\mathrm{MnO}_{6}$ octahedra are edge-shared to one another and corner-shared to the tungsten oxide octahedra they sit between [133, 158]. However, it appears in the hybrids that one of the oxygen atoms is missing from the $\mathrm{MnO}_{6}$ octahedra, forming a square-based pyramid. The next nearest atom is another manganese, and there are either one (i.e. $\mathrm{Mn}$ dimers) or two (1D chains). However, only these two shells were used in the fit and there may be other $\mathrm{Mn}-\mathrm{Mn}$ bonds relatively close which would appear in subsequent shells (as well as other $\mathrm{Mn}-\mathrm{W}$ and $\mathrm{Mn}-\mathrm{O}$ neighbours).

In the absence of single crystal XRD structural analysis, we combine the results presented so far to propose a structure for the inorganic layer. Given the results from the EXAFS analysis, XRD inorganic layer spacing $\left(2 \times \mathrm{WO}_{6}\right.$ octahedra), and the tungsten atom 'stripes' seen in the high-resolution TEM image, a structure is proposed in Figure 5.22 for the inorganic layer.

The proposed structure has the necessary bilayer of $\mathrm{WO}_{6}$ octahedra, edge-shared in the direction normal to the page. In the horizontal direction the structure alternates between lines of tungsten octahedra and manganese metal ions (filled circles in the diagram). The manganese ions are co-ordinated to five oxygen atoms - two from the $\mathrm{WO}_{6}$ octahedra on each 


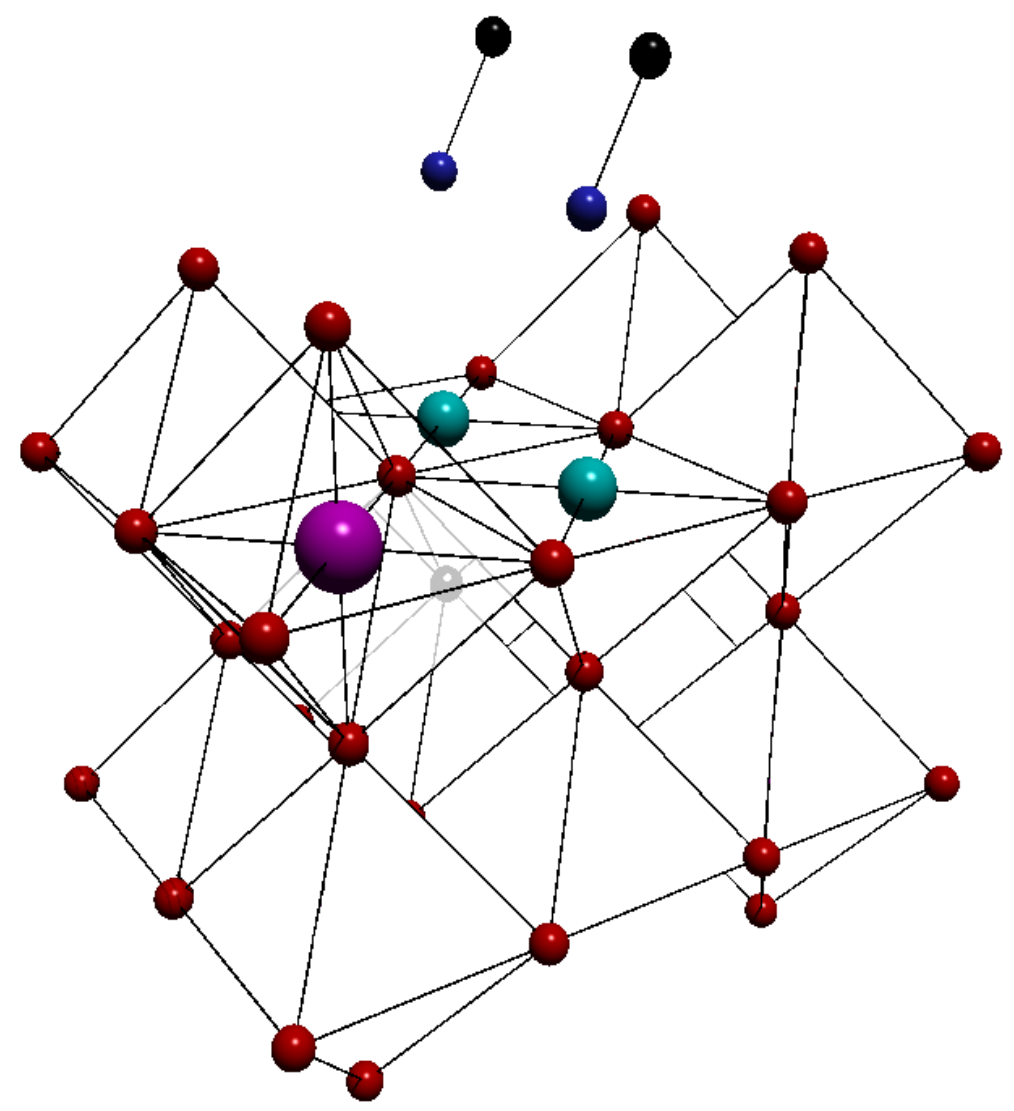

Figure 5.22: Proposed structure for (Mn,W)-DAn, showing a bilayer of $\mathrm{WO}_{6}$ octahedra, $\mathrm{MnO}_{5}$ inverted square pyramids, and the terminal $\mathrm{N}$ and first $\mathrm{C}$ atoms of the organic molecule ( $\mathrm{H}$ atoms not shown for clarity). 
side, and one between them (open circle). The bilayer structure would also mean that if every open transition metal site was occupied by an organic amine termination, the $\mathrm{MWO}_{4}$ :organic ratio would be 2:1 (as there are two $\mathrm{MWO}_{4}$ formula units per organic bidentate molecule), as observed in the microanalysis results (Section 5.2.3).

Past the first two well-defined $\mathrm{W}-\mathrm{O}$ shells (with the two shorter bonds corresponding to the vertical direction and the four longer bonds corresponding to the horizontal), this structure would exhibit $3 \mathrm{~W}-\mathrm{W}$ and $2 \mathrm{~W}-\mathrm{Mn}$ shells at approximately the same distance, which would be difficult to resolve. One would also expect to see $6 \mathrm{~W}-\mathrm{O}$ shells corresponding to the distance between a tungsten atom and an apical oxygen atom on its neighbouring $\mathrm{WO}_{6}$ octahedron or $\mathrm{MnO}_{5}$ square pyramid.

The manganese spectrum would also show $\mathrm{Mn}-\mathrm{Mn}$ and $\mathrm{Mn}-\mathrm{W}$ shells at approximately the same distance for neighbours in-plane, and perhaps a different (shorter) Mn-Mn length in the vertical direction. Hence, while only one Mn-Mn shell is observed (at $3.41 \AA$ ), the next shell to be fitted may correspond to the two longer $\mathrm{Mn}-\mathrm{Mn}$ in-plane neighbours.

We have also performed bond-valence sums on the EXAFS data leading to the proposed structure, following Ref. [2] and [34]. For the $j^{\text {th }}$ atom, its valence $V_{j}$ is given by

$$
V_{j}=\sum_{i} \exp \left(\left(r_{0}-r_{i j}\right) / B\right)
$$

where $r_{0}$ is given in tables in Ref. [34] for many inorganic bonds and $B$ takes a relatively constant value of 0.37 for most systems [34].

For $\mathrm{Mn}$ in the proposed structure we calculate $V_{M n}=2.03 \pm 0.03$, in good agreement with the expected value of +2 as given by the XANES spectra. For $\mathrm{W}$ we calculate $V_{W}=5.56 \pm 0.33$, which is not as good, but is within the uncertainty for $\mathrm{W}^{6+}$ of \pm 0.85 , as calculated from reference [2]. The discrepancy may also be due to uncertainty in fitting the first maximum in the EXAFS spectrum, which normally would arise from three pairs of $\mathrm{W}$ $\mathrm{O}$ shells at slightly different distances. Instead we have 2 short and 4 long distances. 
Thus we conclude the proposed structure is viable from a consideration of the bond valence sums for Mn and W.

Within the proposed structure, the most likely site for the amino terminal group to bind to (or at least, interact with) the inorganic layer is in the open space near the transition metal site. In $\mathrm{MgWO}_{4} \cdot 2 \mathrm{H}_{2} \mathrm{O}$, one of the few metal tungstate hydrates for which a structure has been reported, the oxygen atom of the water molecule forms one apex of each $\mathrm{MgO}_{6}$ octahedron [3, 104]. On the other hand, $\mathrm{MnWO}_{4}$ thin films used as humidity sensors indicate that the $\mathrm{W}$ site is more active for water adsorption [224]. However from our infrared and Raman results (Section 5.2.4) it is clear that the structure formed in the manganese tungstate hydrate and hybrids is not completely related to that of $\mathrm{MnWO}_{4}$. If our proposed structure is correct, the bare manganese ion will be the most reactive site for binding intercalated molecules to, rather than the tungsten octahedra. The apical $\mathrm{W}=\mathrm{O}$ bond would therefore be stronger because it is not affected as much by the amino group as for the tungsten oxide hybrids. This is evident in the Raman spectra (Section 5.2.4) as the corresponding peak shifts to higher energies (higher frequencies). This will play a large part in the other transition metal tungstates, particularly the cobalt, nickel and copper samples, which are known to form amino complexes relatively easily. This is explored in the next section.

\subsubsection{UV-visible spectroscopy}

Many transition metal compounds, especially complexes, exhibit metal-ligand or ligand-metal charge transfer interactions which result in the absorption or emission of visible light. This results in the bright colours often seen in transition metal compounds, and these transition metal tungstate hybrids are no exception. Figures 5.23, 5.24 and 5.25 show the UV-visible powder diffuse reflectance performed on lightly pressed samples as described in Chapter 2. Reference [166] was used extensively in the interpretation of the spectra.

The manganese samples (Figure 5.23a) both display a single absorption edge, the midpoint of which lies at $387 \mathrm{~nm}$. In appearance they are quite similar to the spectra of the W-DAn hybrids (Section 4.7.1) in that the position 


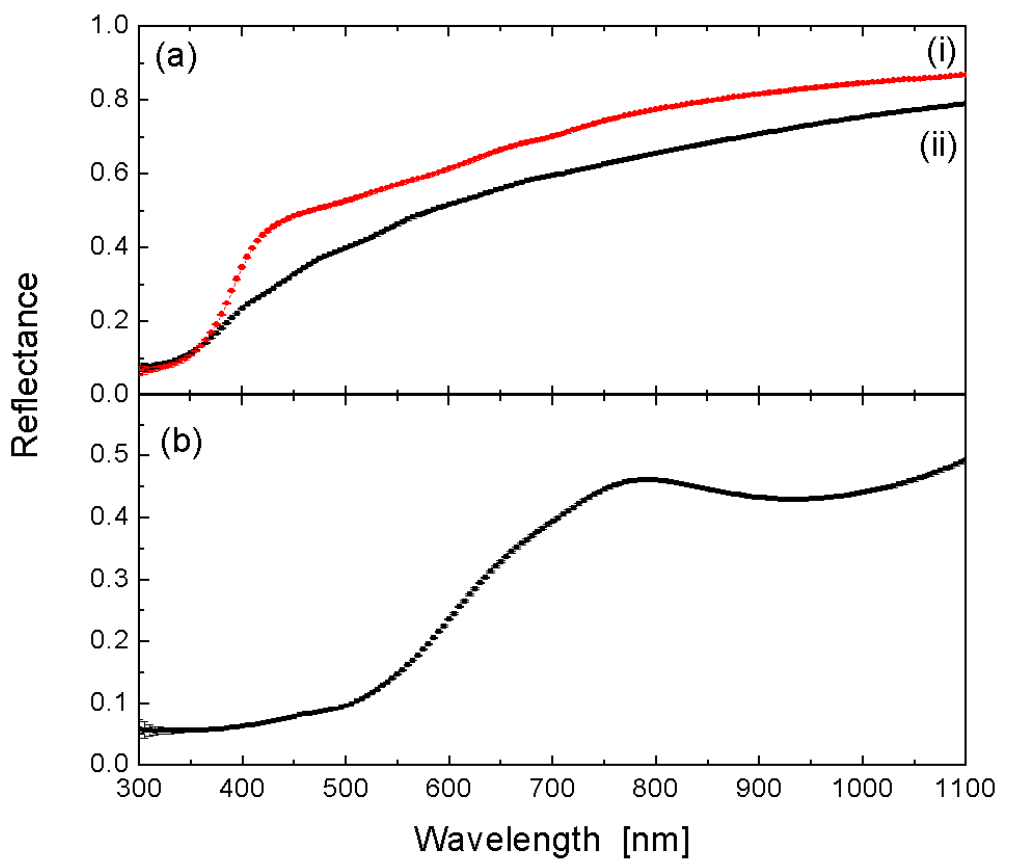

Figure 5.23: UV-visible spectra of (a) Mn tungstate hybrids: (i) (Mn,W)DA2, (ii) (Mn,W)-DA6; and (b) Fe tungstate hybrids: (Fe,W)-DA12

of the absorption edge appears to be independent of the alkyl length (and hence interlayer spacing), indicating that adjacent layers are electronically decoupled. The energy that the edge corresponds to is $3.20 \mathrm{eV}$, lower than that of the W-DAn hybrids. Some slight changes in gradient at 480, 565 and $685 \mathrm{~nm}$ may correspond to charge transfer transitions [166, p.449], however their low intensity indicates that while the manganese in the hybrid structure may be interacting with the organic molecule in a complex-like manner, this is much weaker than the interaction between the manganese and the tungsten and oxygen atoms nearby, forming the inorganic layer. It behaves more like a traditional semiconductor with a band gap than a complex incorporated within the inorganic layer.

The manganese samples are all of a similar light brown colour, regardless of the length of the organic intercalate. Manganese (II) compounds in the high spin state often exhibit a weak pink colour, which is consistent with the colouring of these samples [166, p.448].

Similarly the iron samples are all of a similar orange-brown colour and 


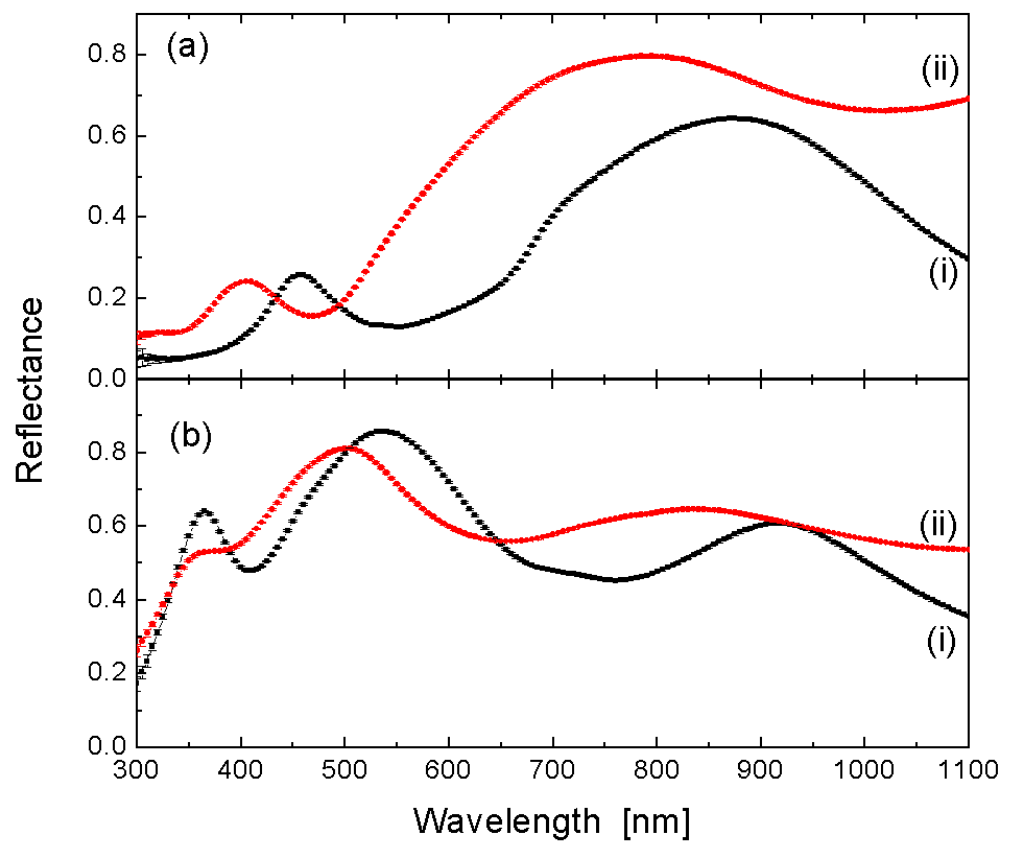

Figure 5.24: UV-visible spectra of (a) Co tungstate hybrids: (i) $\mathrm{CoWO}_{4}$. $n \mathrm{H}_{2} \mathrm{O}$, (ii) (Co,W)-DA2; and (b) Ni tungstate hybrids: (i) $\mathrm{NiWO}_{4} \cdot n \mathrm{H}_{2} \mathrm{O}$, (ii) $(\mathrm{Ni}, \mathrm{W})-\mathrm{DA} 6$

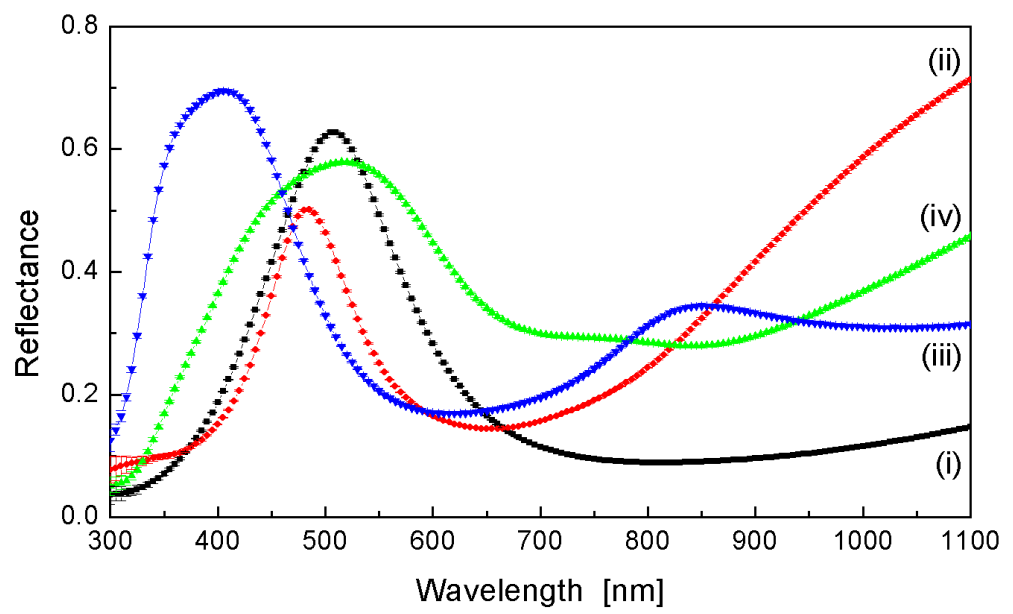

Figure 5.25: UV-visible spectra of $\mathrm{Cu}$ tungstate hybrids: (i) $\mathrm{CuWO}_{4} \cdot n \mathrm{H}_{2} \mathrm{O}$, (ii) $(\mathrm{Cu}, \mathrm{W})-\mathrm{DA} 2$, (iii) $(\mathrm{Cu}, \mathrm{W})-\mathrm{DA} 6$, (iv) $(\mathrm{Cu}, \mathrm{W})$-phen. 
display similar UV-visible spectra to that of (Fe,W)-DA12 shown in Figure $5.23 \mathrm{~b}$. This spectrum shows a single broad feature, peaking at $788 \mathrm{~nm}$ and remaining high to lower energies. It appears that this is an absorption edge with a peak feature, corresponding to semiconducting behaviour with additional Fe-ligand influence. In this case the Fe-ligand interaction is stronger than the Mn-ligand interaction. The value of $788 \mathrm{~nm}$ is similar to values of 710-900 nm reported for iron-diaminoalkane complexes [166, p.460].

Manganese and iron are the only two of the five transition metal hybrid series studied which were able to be formed from aqueous ammonia solution, using the preferred 'solution method'. Cobalt, nickel and copper all prefer to form ammonia complexes over the tungstate hybrid precipitate. It is not surprising then, that this complex-like behaviour is observed in the UV-visible spectra. While the metal ions are bound within the inorganic layers, the interaction between them and the organic amine termination is significant. This is now discussed for the three series in question.

The cobalt tungstate hybrids studied are shown in Figure 5.24a. They show two peak features: a small sharp peak near $400 \mathrm{~nm}$ and a large broad peak near $800 \mathrm{~nm}$. The peaks of the (Co,W)-DA2 compound lie at lower wavelengths (higher energies) than $\mathrm{CoWO}_{4} \cdot n \mathrm{H}_{2} \mathrm{O}$.

For octahedral high spin cobalt (II) complexes, two main absorption bands are observed at 1000-1250 $\mathrm{nm}$ and $500 \mathrm{~nm}$ [166, p.480]. In complexes of cobalt (II) with ammonia or organic amines, these values decrease in wavelength [166, p.481]. For tetrahedral cobalt (II), the absorption bands occur at 1110-2000 nm and 550-670 nm [166, p.496-498]. This difference is fairly strong evidence that the cobalt ions are present in the octahedral configuration, as they are in $\mathrm{CoWO}_{4}$.

We proposed that the environment of the transition metal ion in the transition metal tungstate hybrids forms a square pyramid with 5 oxygens of the tungsten oxide octahedra, with the apex of the pyramid within the inorganic layer. This leaves the metal ion open to form a complex-like interaction with the terminal amino group of the organic molecules. Hence it is not surprising 
that the optical spectra resembles that of a cobalt-amino complex, shifted to higher energies because the cobalt is bound within the inorganic layer. The extra binding increases the energy required for charge transfer to the ligand to occur.

In the nickel tungstate hybrids (Figure 5.24b), three absorption bands are observed at $370 \mathrm{~nm}, 500-530 \mathrm{~nm}$ and $830-930 \mathrm{~nm}$. These are comparable with the observed ranges for the three spin-allowed transitions in many octahedral nickel (II) compounds: 370-520 nm, 500-910 nm and 770-1430 nm [166, p.507]. As for the cobalt samples, the observed transitions of the nickel hybrids lie at the high-energy end of these ranges, as in fact do many of the nickel-amine complexes (e.g. ethylenediamine, pyridine, bipyridine) [166, p.508]. Tetrahedral nickel complexes on the other hand display one major band near $670 \mathrm{~nm}$ [166, p.530]. Clearly the spectra observed correspond to nickel (II) in an octahedral environment.

Lastly, the copper tungstate hybrids, shown in Figure 5.25, display for the most part a single absorption band near $500 \mathrm{~nm}$. In $(\mathrm{Cu}, \mathrm{W})-\mathrm{DA} 2$ the prominent band is shifted to higher energies (400 nm) and an additional small peak feature is seen at $850 \mathrm{~nm}$. Jahn-Teller distortion of copper ions causes features in the optical spectra to broaden out, and most studies of copper complexes report a single poorly-resolved broad band [166, p.555]. While copper (II) octahedral complexes are rare (copper prefers square planar symmetry), typical values are in the range 560-670 nm [166, p.557]. Square planar copper complexes, especially to ammonia/amino ligands, have been observed to have absorption bands as low as $500 \mathrm{~nm}[166, \mathrm{p} .572]$.

A summary of the UV-visible results is as follows: it appears the samples can be divided into two groups based on the spectra - Mn and Fe hybrids, and $\mathrm{Co}, \mathrm{Ni}$ and $\mathrm{Cu}$ hybrids. The former samples are able to be formed via the solution method, an initial indication that their covalent interaction within the inorganic tungstate layer is greater than any affinity for the ammonia solution or amino organic molecules. Their optical spectra show a single absorption edge as opposed to a series of bands, indicating that their electronic properties are similar to those of the tungsten oxide hybrids, with a more or 
less well-defined band gap.

The latter samples (Co, $\mathrm{Ni}$ and $\mathrm{Cu}$ hybrids) however, display optical spectra reminiscent of their amino complexes. The increased affinity for these transition metals to form such complexes is observed in the comparison of synthesis techniques, where it was noted that these hybrid materials cannot be formed in ammonia solution. Therefore one might expect complex-like behaviour of the metal-organic interaction in the hybrid material itself, particularly if the bare metal ion site is the preferred site for the organic amine to bind to. In this case the charge transfer between the metal and the terminal amino groups dominates the observed optical spectra. Often the energy is increased relative to that expected, because the metal is also bound within the inorganic tungstate layer.

\subsection{Summary}

The transition metal tungstates have been characterised using a number of techniques to probe their structural, chemical, and electronic properties.

In this work, the manganese tungstates have been the most extensively studied of the five transition metal hybrid series, due to a number of factors. They are able to be synthesised via the preferred solution-based method, which results in better crystallinity of the final powder product. This in turn gives better results in XRD and TEM, the primary means used in absence of single crystal XRD for determining crystal structure features. As for the 'parent' tungsten oxide hybrids, there is evidence for a layered structure, in this case built on a bilayer of oxide species. From TEM images and EXAFS analysis it is inferred that the oxide structure consists of alternating 'stripes' of $\mathrm{MnO}_{5}$ square pyramids joined at their apices, and $\mathrm{WO}_{6}$ octahedra. The organic molecules are tilted at an angle of $56.5^{\circ}$ to the inorganic layer. XANES spectra clearly show that manganese is present as $\mathrm{Mn}^{2+}$ and tungsten as $\mathrm{W}^{6+}$. A combination of microanalysis and EDX confirms the stoichiometry of this arrangement. Because of the more open nature of the structure, particularly along the manganese stripes where there are no apical oxygen atoms, it should in principle be possible to include somewhat more 'bulky' 
molecules than can be included in the 'parent' tungsten oxide system. Indeed, a sample was successfully synthesised using DAphen (1,4-diaminobenzene, $\left.\mathrm{H}_{2} \mathrm{~N}\left(\mathrm{C}_{6} \mathrm{H}_{4}\right) \mathrm{NH}_{2}\right)$ as the organic molecule.

Again, as for the 'parent' compound, electron diffraction reveals the presence of a supercell, which has confounded efforts to solve the crystal structure directly from Rietveld refinement of the powder XRD data. Unlike the 'parent' compound series, the infrared spectra of the (Mn,W)-DAn series indicates an absence of $-\mathrm{NH}_{3}{ }^{+}$terminal groups. This is not surprising, as the inorganic layer, $\mathrm{Mn}^{2+} \mathrm{W}^{6+} \mathrm{O}_{4}$, has zero net charge.

Cobalt, nickel and copper hybrids on the other hand display a different chemistry than manganese, as evidenced from their UV-visible spectra. They each display a number of absorption bands in the visible spectrum (rather than an absorption edge), indicating that the interaction between the organic amino group and the transition metal ion may resemble that of an amino complex. The (M,W)-DA2 compounds of each of these appear to form a completely different structure altogether. 


\section{Chapter 6}

\section{Magnetic Properties}

The incorporation of unpaired spins into the transition metal hybrid structures should lead to magnetically correlated states, depending upon the strength of coupling between the spins both within the inorganic layer and between layers. The layered organic-inorganic hybrid systems have the potential, particularly using insulating saturated amino-alkanes as the organic intercalates, to represent a nearly-perfect two-dimensional magnetic system. Models for such low-dimensional systems have been developed in the past (e.g. [?]), however there are relatively few experimental compounds to apply them to. Hence the magnetism of the layered organic-inorganic hybrids, particularly those incorporating magnetic ions, represents an area of physics where the enforced low-dimensionality causes severe anisotropy in the properties of the material.

The samples were measured with a SQUID magnetometer as described in Section 2.4.

This chapter begins by summarising the major types of magnetic behaviour, as outlined in Ref. [192].

\subsection{Introduction to magnetic behaviour}

Two means used to describe a material's magnetic behaviour are to observe the temperature-dependence and field-dependence of the magnetisation $M$ (i.e. $M(T)$ and $M(H)$ respectively). The vast majority of materials display 
linear, reversible $M(H)$ behaviour, which is a characteristic of paramagnetism and arises from the moments associated with localised unpaired electrons. As the field increases, the alignment and hence the measured moment also increases proportionally. The ratio $M / H$ is called the magnetic susceptibility, $\chi$.

If the atoms with the localised unpaired electrons do not interact with each other, the system will obey the Curie law, $\chi=C / T$ where $C$ is the Curie constant. The $1 / T$ behaviour arises as thermal motion of the electrons disrupts the alignment of the spins parallel to the applied field. The Curie constant is related to the number of unpaired electrons in the system.

When adjacent moments in a paramagnetic system interact, the exchange interaction can assist to align the moments either parallel or anti-parallel. This introduces a new term into the equation for Curie behaviour, and we obtain the Curie-Weiss law, $\chi=C /(T-\Theta)$ where the new parameter $\Theta$ is the Weiss temperature, which is related to the strength and nature of the exchange interaction. If $\Theta$ is positive, adjacent moments align in the same direction, as a result of ferromagnetic (FM) correlations. If $\Theta$ is negative, adjacent moments align in opposite directions, as a result of antiferromagnetic (AF) correlations. While $\Theta$ is not strictly a physical property, in FMcorrelated systems there is an additional transition often near $T=\Theta$ (where the Curie-Weiss law diverges), and the transition temperature is called the Curie temperature, $T_{C}$. In AF-correlated systems there may be a transition near $T=|\Theta|$, and the transition temperature in these systems is called the Néel temperature, $T_{N}$.

Pauli paramagnetism and Van Vleck paramagnetism are two examples of temperature-independent paramagnetism. Pauli paramagnetism arises in metals, where the conduction electrons can be aligned by the applied magnetic field. Van Vleck paramagnetism arises due to thermal excitations of electrons to low-lying excited states. Both contributions are small and normally treated as a temperature-independent constant. The constancy of the Pauli paramagnetism arises in turn from a more or less constant density of states (DOS) near the Fermi level. If the DOS is strongly energy-dependent, as for example near a van Hove singularity, then the pauli susceptibility will 
be temperature-dependent.

Paired electrons often give rise to diamagnetism, where the $M(H)$ behaviour is linear and reversible, but has a negative slope (i.e. $\chi$ is negative). In most systems this is very small and temperature-independent. The core electrons can also contribute a very small diamagnetic moment.

Unlike paramagnetic and diamagnetic systems, ferromagnetic systems display non-linear, irreversible $M(H)$ curves $^{1}$. In ferromagnetic systems the spins interact strongly with each other and align parallel to the applied field, resulting in a large saturation magnetisation (one parameter used to describe ferromagnetic systems). The $M(T)$ behaviour obeys the Curie-Weiss law at temperatures far above the Curie temperature $T_{C}$, below which the ferromagnetic phase is formed, and $\chi(=M / H)$ becomes field-dependent as $M / H$ is non-linear in this regime. Magnetic correlations lead to a departure from linear $\mathrm{M}(\mathrm{H})$ near but above $T_{C}$, where $\chi \propto\left(T-T_{C}\right)^{-n}$ (for 3-dimensional systems).

The irreversibility, or hysteresis, in the $\mathrm{M}(\mathrm{H})$ loops at $T<T_{C}$ is also evident in the $\mathrm{M}(\mathrm{T})$ dependence and can be detected by performing two temperature scans, one where the field is applied at low temperatures after cooling in zero field ('zero-field-cooled' or ZFC), and one where the sample is cooled while the field is turned on, and then measured in the same field upon warming ('field-cooled' or FC). A comparison of the two curves indicates the temperature range over which the system displays irreversible behaviour.

In antiferromagnetic systems there is also long-range ordering, however adjacent spins align in opposite directions. While the $M(H)$ behaviour at all temperatures resembles that of a paramagnet, the AF behaviour is attributed to long-range order. $M(T)$ plots often display a cusp-shaped feature characteristic of an AF transition at the Nèel temperature, $T_{N}$. Above $T_{N}$ they display Curie-Weiss dependence with a negative Weiss temperature.

Ferrimagnetism is a phenomenon that occurs when spins are antiferro-

\footnotetext{
${ }^{1}$ For systems with extremely small grains (e.g. Fe nanoparticles) there are no domain wall boundaries and the $M(H)$ curves are reversible.
} 
magnetically aligned, but adjacent moments are of different magnitude, resulting in a net ferromagnetic component. Ferrimagnetic materials have similar $M(H)$ and $M(T)$ behaviour to ferromagnets, although the magnetisation is not as large. Ferrimagnetism can only occur in systems where atoms with multiple different spins are ordered (e.g. $\mathrm{Fe}_{3} \mathrm{O}_{4}$, which has two ordered spin states of iron, $\mathrm{Fe}^{2+}(S=2)$ and $\left.\mathrm{Fe}^{3+}\left(S=\frac{5}{2}\right)\right)$, or materials with two ordered spin systems of different orientations.

Another way to observe a net ferromagnetic moment in an antiferromagnetically ordered system is if the spins are tilted (or canted) slightly in an orthogonal direction. See for example reference [24]. Like ferrimagnets, spin-canted antiferromagnets exhibit FM-like behaviour in $M(T)$ and $M(H)$, albeit with much smaller magnetisations than if the system was purely ferromagnetic. If the field is applied in the plane of (and aligned with) the ordered moments, no magnetisation is observed up to a threshold field where the AF moments then flip to an orientation orthogonal to the field, when they may cant. This is known as a spin-flop transition and is characterised by a magnetisation loop resembling a figure-8: the moment is zero up to the spin-flop field, then rises sharply. On reversal the magnetisation collapses back to zero when the field is low enough for the moments to realign. The reverse cycle shows the same behaviour at negative moment.

As might be expected, very few materials have completely non-interacting spins. In low-dimensional materials the exchange interactions along chains or within planes are expected to be much stronger than between chains or planes. These considerations give rise to a number of models, which we describe next.

\subsection{Low-dimensional models}

The spin Hamiltonian is given in the general form

$$
\mathcal{H}=-2 J \sum_{i>j}\left[a S_{i}{ }^{z} S_{j}{ }^{z}+b\left(S_{i}{ }^{x} S_{j}{ }^{x}+S_{i}{ }^{y} S_{j}{ }^{y}\right)\right]
$$


where $i$ and $j$ are nearest-neighbour spins and $J$ is the exchange constant [71].

When the interaction is completely isotropic (direction-independent), $a=$ $b=1$ in equation 6.2 and we obtain the 3D Heisenberg model:

$$
\mathcal{H}=-2 J \sum_{i>j} \mathbf{S}_{i} \cdot \mathbf{S}_{j}=-2 J \sum_{i>j} S_{i}^{x} S_{j}{ }^{x}+S_{i}{ }^{y} S_{j}{ }^{y}+S_{i}{ }^{z} S_{j}{ }^{z}
$$

On the other hand, if the interaction is completely anisotropic, we obtain the Ising model, with $a=1$ and $b=0$ :

$$
\mathcal{H}=-2 J \sum_{i>j} S_{i}{ }^{z} S_{j}^{z}
$$

In the case where $a=0$ and $b=1$ we obtain the planar Heisenberg or $\mathrm{XY}$-model (providing the spins are contained within the $x y$-plane):

$$
\mathcal{H}=-2 J \sum_{i>j}\left(S_{i}^{x} S_{j}^{x}+S_{i}^{y} S_{j}^{y}\right)
$$

The models can be extended further, by considering next-nearest neighbours, and also by using fractional values of $a$ and $b$. Different models are obtained for different dimensionality of systems - for example, dimers and tetramers [229], 1D chains [218, 229, 165, 190], 'spin ladder' chains [281], triangular 2D lattice [117, 119], in addition to the 2-dimensional Ising and Heisenberg models already discussed. Models exist for both antiferromagnetically ordered and ferromagnetically ordered systems. It is also possible to observe different ordering within, versus between, layers (e.g. $J_{\|}>0$ (FM) while $J_{\perp}<0(\mathrm{AF})$, ref. [119]).

The Heisenberg and XY- models do not predict long-range ordering with an associated phase transition at finite temperatures [250, 71]. Hence any transitions observed in the magnetisation temperature-dependance are due either to interplane interaction (phase transitions, extra-dimensional coupling) and/or some anisotropy (Ising component).

At higher temperatures, the temperature-dependence of the magnetisation given by the models reduces to Curie-Weiss behaviour, given by 


$$
\chi=\frac{C}{T-\Theta}+T I P
$$

where $C$ is the Curie constant, $\Theta$ is the Curie-Weiss temperature and TIP is any remnant temperature-independent paramagnetic component (e.g. from Pauli and/or Van Vleck paramagnetism and/or core diamagnetism). In general, a material with a negative Weiss temperature orders antiferromagnetically, while a material with a positive Weiss temperature orders ferromagnetically. (There are some exceptions, for example in the $\mathrm{RuSr}_{2} \mathrm{GdCu}_{2} \mathrm{O}_{8}$ system, $\Theta$ is positive but the dominant ordering is antiferromagnetic [36, 185].)

It is common practise in many journal articles reporting preliminary magnetisation data to determine from the Weiss temperature $\Theta$ whether the system displays ferromagnetic (FM) or antiferromagnetic (AF) correlations. Such magnetisation analysis is normally presented either as $1 / \chi$ vs. $T$ or as $\chi T$ vs. $T$. Many of the models for $1 \mathrm{D}$ chains etc. give a $\chi$ vs. $T$ plot which closely resembles a $1 / T$ like behaviour, however only in $\chi T$ or $1 / \chi$ vs. $T$ plots can the differences be clearly seen.

Unfortunately it is not a trivial exercise to obtain a model for the $\chi(T)$ dependence from the spin Hamiltonian derived from the crystal structure. These models also do not allow for phase transitions or coupling at low temperatures.

Most transition metal ions have relatively little anisotropy due to strong crystal field quenching and therefore can generally be described using a Heisenberg model. Cobalt is an exception, often displaying significant anisotropy [286].

We now present and discuss the magnetisation results obtained for the various hybrid materials.

\subsection{Tungsten oxide hybrids}

Tungsten oxide hybrids are not expected to exhibit any magnetism because the tungsten is present as $\mathrm{W}^{6+}$, and hence has spin $S=0\left(5 \mathrm{~d}^{0}\right)$. Figure 6.1 shows a temperature sweep of W-DA6 in a field of $1 \mathrm{~T}$, along with the 


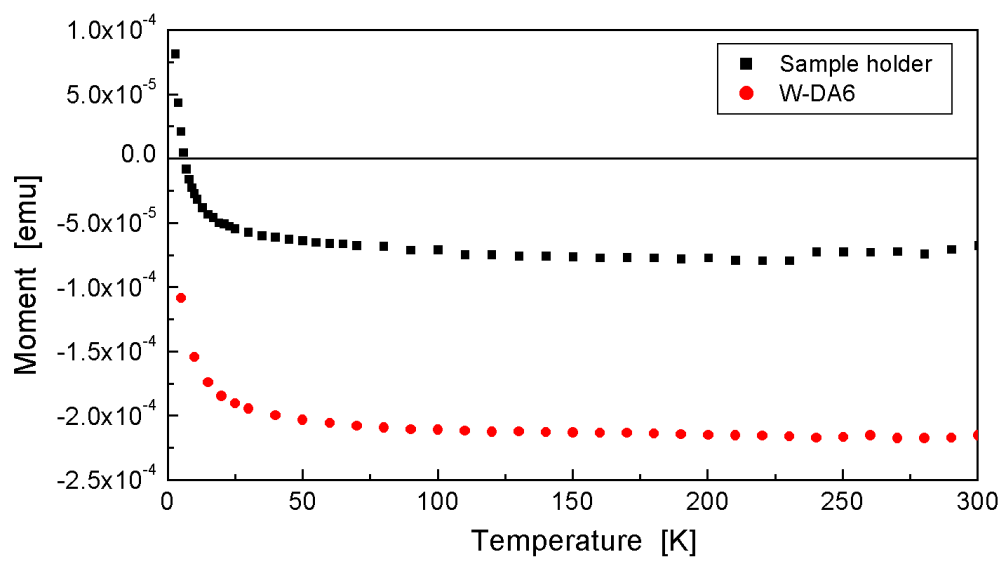

Figure 6.1: DC magnetisation of W-DA6, compared with the sample holder only, measured at $1 \mathrm{~T}$.

signal observed for the sample holder alone. The difference between the two curves corresponds to the very weak diamagnetism observed in systems with filled orbitals and hence paired electrons. Hence we can also be sure that magnetism observed in the transition metal tungstate hybrids arises solely from the arrangements of the transition metal ions, and not from the tungsten or any interaction between the transition metal and tungsten ions.

\subsection{Transition metal tungstate hybrids}

A wide variety of different behaviours are exhibited by the various transition metal tungstate hybrids. Many samples display features corresponding to crossovers from $2 \mathrm{D}$ to $3 \mathrm{D}$ magnetic behaviour. In all cases the hightemperature part of the DC susceptibility can be approximated by the CurieWeiss Law (Eq. 6.2):

$$
\chi=\frac{C}{T-\Theta}+T I P
$$

Typical Weiss temperatures for some of the transition metal tungstate hybrids studied are given in Table 6.1.

From the Curie constant $C$, one can determine the effective moment, $P^{e f f}$, contributed by the transition metal ion present, from the formula 


\begin{tabular}{|c|c|c|c|c|c|}
\hline & $\mathrm{Mn}$ & $\mathrm{Fe}$ & $\mathrm{Co}$ & $\mathrm{Ni}$ & $\mathrm{Cu}$ \\
\hline \hline $\mathrm{MWO}_{4}$ & -72.8 & & -98.1 & & \\
$(\mathrm{M}, W)-\mathrm{H}_{2} \mathrm{O}$ & -16.6 & -23.4 & -1.0 & 6.8 & -2.8 \\
(M,W)-DA2 & -19.6 & -9.5 & -0.1 & -0.2 & -0.3 \\
(M,W)-DA6 & -19.6 & -3.7 & -17.6 & 6.3 & 0.3 \\
(M,W)-DA12 & -20.1 & -4.4 & & & \\
(M,W)-phen & -19.7 & & & -0.9 & 3.3 \\
\hline
\end{tabular}

Table 6.1: Weiss temperatures (in Kelvin) for transition metal tungstate hybrids.

$$
C=\frac{1}{3} \frac{N}{V} \frac{\mu_{B}^{2} \mu_{0}\left(P^{e f f}\right)^{2}}{k_{B}} \quad \text { (SI units) }
$$

where $N$ is the number of magnetic ions per cell, $V$ is the cell volume, and $\mu_{B}, \mu_{0}$ and $k_{B}$ are the Bohr magneton, permeability of vacuum and Boltzmann constants respectively. This can be compared with the expected value calculated from the spin of the ion, $P^{e f f}=g \sqrt{S(S+1)}[9]$. Figure 6.2 illustrates the good agreement between the experimental values calculated from equation 6.4, literature values for transition metal tungstate systems [266], and the expected theoretical values [9].

We now examine some of the more interesting samples in greater detail.

\subsubsection{Manganese tungstate hybrids}

The DC susceptibility of a series of (Mn,W)-DAn hybrids is shown in Figure 6.3. The field- and zero-field-cooled curves are identical, indicating that the magnetisation is reversible. There is no field-dependence of the susceptibility $(\propto M / H)$ for applied fields up to $1 \mathrm{~T}$.

In each case there is a transition at low temperatures, indicated by the arrows. For $(\mathrm{Mn}, \mathrm{W})-\mathrm{DA} 2$ and $(\mathrm{Mn}, \mathrm{W})-\mathrm{DA} 6$ especially, the feature is cusplike, characteristic of an antiferromagnetic ordering transition. (The clarity of the transition in these two samples is probably due to their increased crystallinity compared with the longer chain alkane hybrids.)

The Néel temperature is plotted as a function of alkyl chain length in the 


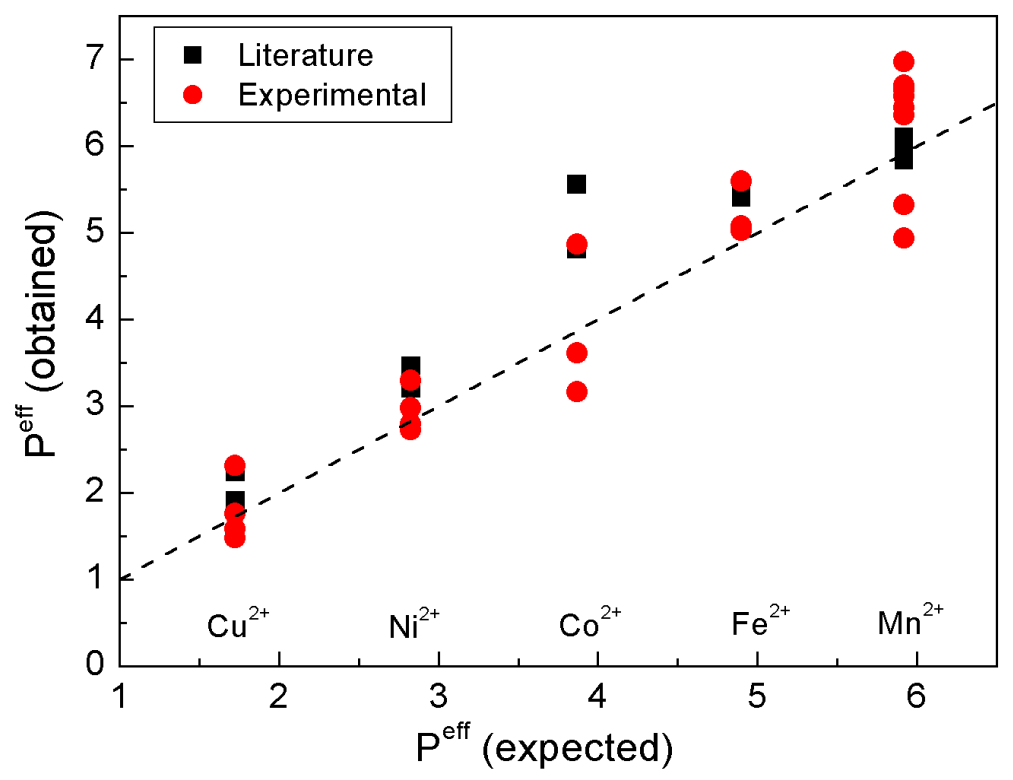

Figure 6.2: Experimental values of $P^{e f f}$ for transition metal tungstate hybrids, compared with literature and theoretical values.

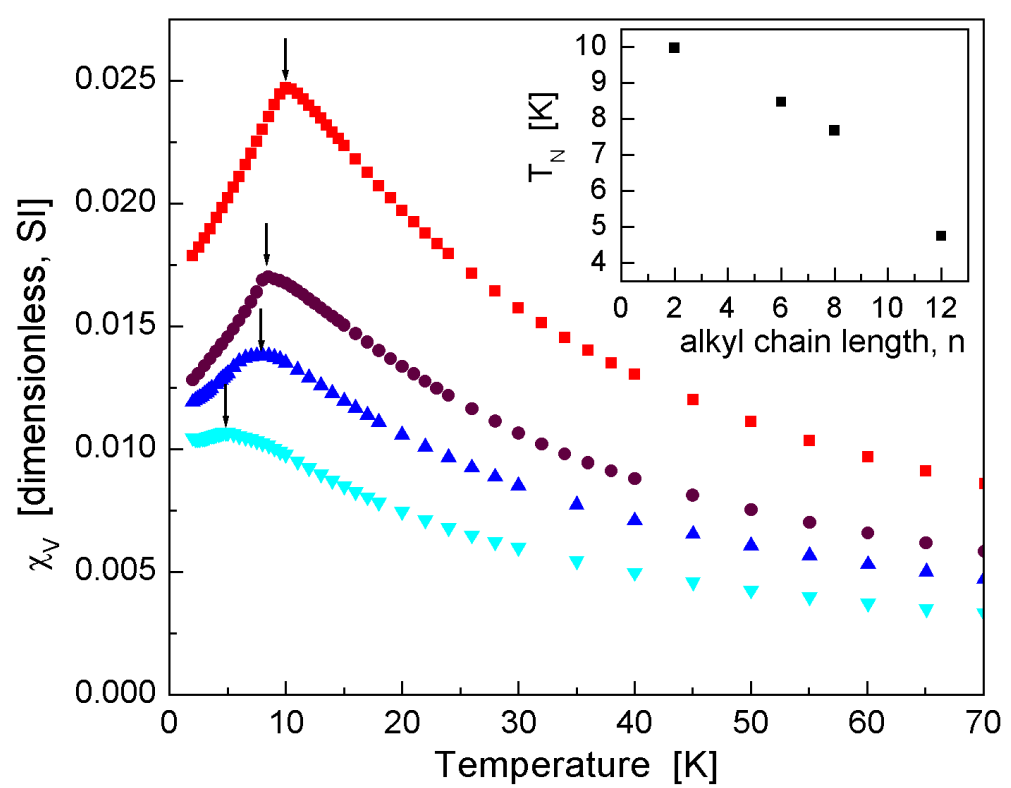

Figure 6.3: DC susceptibility of (Mn,W)-DAn series (top to bottom: $\mathrm{n}=2$, $6,8,12) . T_{N}$ is indicated by the arrows and plotted in the insert versus alkyl chain length. 


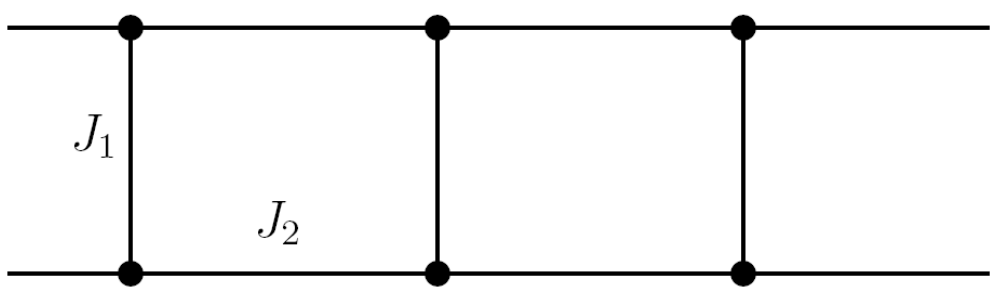

Figure 6.4: Diagram showing the two principle exchanges in a spin ladder: $J_{1}$, the 'rung', and $J_{2}$, the 'rail'.

insert. This shows a monotonic decrease in $T_{N}$ as the alkyl length increases. As evidenced from the XRD results for this series of compounds (Section 5.2.1) the distance between adjacent inorganic layers is proportional to the alkyl chain length. It is expected that as the interlayer spacing is increased the interlayer coupling will decrease, which in turn will cause the transition temperature to decrease. This is qualitatively what is observed.

Curie-Weiss fits to the high-temperature parts of the curves $(T>50 K)$ display consistent Weiss temperatures between -19 to $-20 \mathrm{~K}$ across the DAn series, as shown in Table 6.1. This is in contrast to the 'parent' $\mathrm{MnWO}_{4}$ compound which has a Weiss temperature of $-72 \mathrm{~K}$ [266]. This parent compound is not layered and hence exhibits strong AF ordering in 3 dimensions, which is weakened in the case of the layered hybrid materials.

From the proposed structure in Section 5.2.5, as determined from TEM, EXAFS, and XRD, the arrangement of the manganese ions resembles that of a spin ladder compound. Spin ladders represent one of the more exotic types of 1-dimensional magnetism, with the best-known example being $\mathrm{SrCu}_{2} \mathrm{O}_{3}$ and its extensions, which form ladders of $\mathrm{Cu}-\mathrm{O}$ linked squares $[126,62,63]$. Examples of other spin ladders include (VO) ${ }_{2} \mathrm{P}_{2} \mathrm{O}_{7}[20,64]$ and $\left(\mathrm{C}_{5} \mathrm{H}_{12} \mathrm{~N}\right)_{2} \mathrm{CuBr}_{4}[278,281]$. While a spin ladder model has been derived computationally, it is only valid for those systems with $\operatorname{spin}=\frac{1}{2}$ (e.g. $\mathrm{Cu}^{2+}$ ) [126]. This model has been found to fit experimental $\mathrm{Cu}^{2+}$ spin ladder systems extremely well [281].

Figure 6.4 demonstrates that for spin ladder compounds there are two major exchange interactions, $J_{1}$ (the 'rung') and $J_{2}$ (the 'rail'). In the case where $J_{1} \gg J_{2}$, the model reduces to spin dimers, whereas for $J_{1} \ll J_{2}$ the 


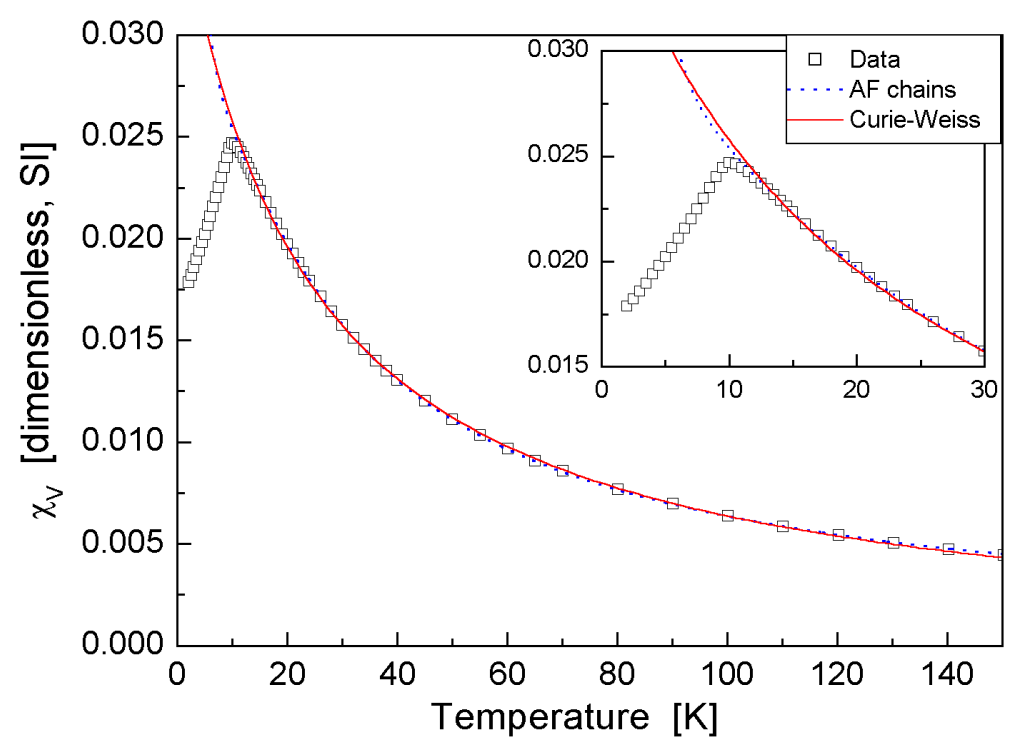

Figure 6.5: DC susceptibility of $(\mathrm{Mn}, \mathrm{W})$-DA2 with best fit curves to the Curie-Weiss and Heisenberg AF chain models (fitted to $T>T_{N}$ ).

model reduces to $1 \mathrm{D}$ chains.

A model for antiferromagnetic Heisenberg chains has been derived for spin $\frac{5}{2}$ systems $[74,83]$. The temperature-dependence of the susceptibility is given by the equation

$$
\begin{array}{r}
\chi=\frac{C}{T-\Theta}\left(\frac{1-u}{1+u}\right)+T I P, \\
u=\frac{T}{T_{0}}-\operatorname{coth} \frac{T_{0}}{T}, \quad T_{0}=\frac{2 J S(S+1)}{k_{B}}
\end{array}
$$

This model resembles the Curie-Weiss law, with an additional temperature-dependent perturbation, where $J$ is the coupling within the chains and $S=\frac{5}{2}$ is the spin of the ion.

The (Mn,W)-DA2 curve is shown in Figure 6.5, with Curie-Weiss and AF chain fits to the data for $T>T_{N}$. The differences are subtle, but the chi-squared degree of fit for the AF chain model indicates a better fit than the Curie-Weiss, and matches the curve almost up to the AF transition (as shown in the insert). 
From the fit parameters, the intrachain coupling value, $J$, has a value of $J / k_{B}=-1.20 \mathrm{~K}$. This is comparable with similar values obtained for layered manganese phosphate hybrids of $-0.79 \mathrm{~K}$ [165] and $-1.66 \mathrm{~K}$ [250].

\subsubsection{Iron tungstate hybrids}

While the manganese tungstate hybrids display relatively uniform behaviour (a single AF transition with a Néel temperature that is affected by the inorganic interlayer spacing), the iron tungstate hybrids' behaviour is somewhat more complicated.

As evidenced in Table 6.1, the (Fe,W)-DAn hybrids all display Weiss temperatures of around -3 to $-10 \mathrm{~K}$, indicating the presence of weak antiferromagnetic correlations. There is greater uncertainty in the Weiss temperatures because of the field-dependence of the DC susceptibilities ${ }^{2}$, as shown in Figure 6.6. The Curie-Weiss fits were performed for temperatures above where the curves at different fields converge, i.e. $T>200 \mathrm{~K}$.

Also clearly evident from the $M / H$ curves shown in Figure 6.6 is the divergence at each field of the $\mathrm{FC}$ and $\mathrm{ZFC}$ data, indicating irreversibility below temperatures of up to $12 \mathrm{~K}$. This divergence temperature is also fielddependent. The divergent behaviour is also slightly different for the three compounds presented here.

In $(\mathrm{Fe}, \mathrm{W})-\mathrm{DA} 2$, the iron hybrid with the shortest alkyl chain studied, the ZFC data passes through an abrupt peak (which is suppressed at higher fields) and then follows a uniform trend in what appears to be a $3 \mathrm{D}$ coupled state. A similar well-defined peak is observed in (Fe,W)-DA12, but without the uniform ZFC trend behaviour. It is thought that the convergence behaviour is limited by $J_{\perp}$, which is overridden as the applied field is increased (indicating that there may be some anisotropy present).

However for the sample with a 6-carbon chain, the ZFC and FC curves separate very gradually and there is no well-defined transition temperature. This is generally expected for compounds where there is a high degree of disorder and hence no well-defined transition temperature.

\footnotetext{
${ }^{2}$ In actual fact, since $M$ is not linear in $H$, it is more proper to call these $M / H$ curves
} 


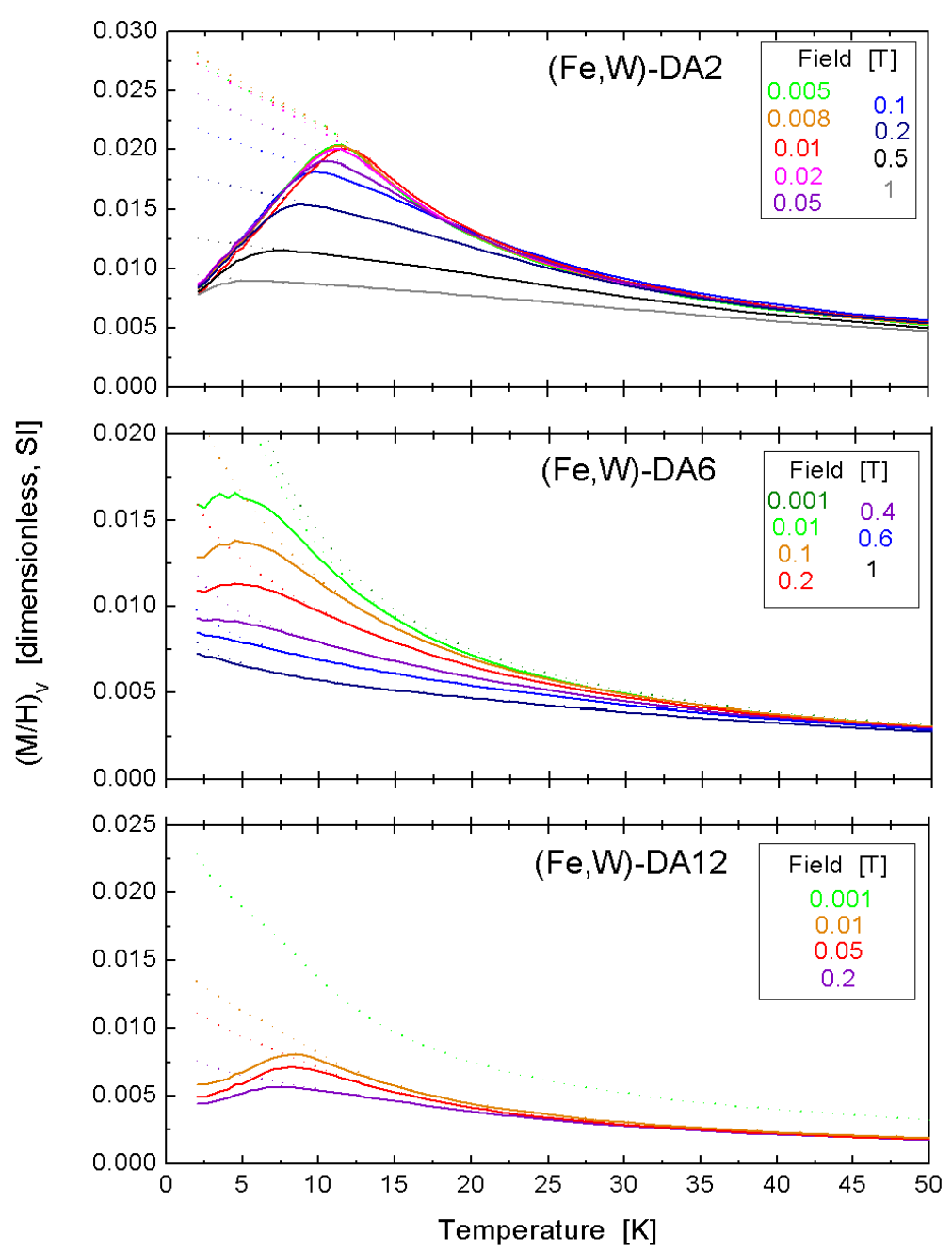

Figure 6.6: $M / H$ curves for $(\mathrm{Fe}, \mathrm{W})$-DAn $(n=2,6,12)$ for applied fields of $0.001-1 \mathrm{~T}$. 


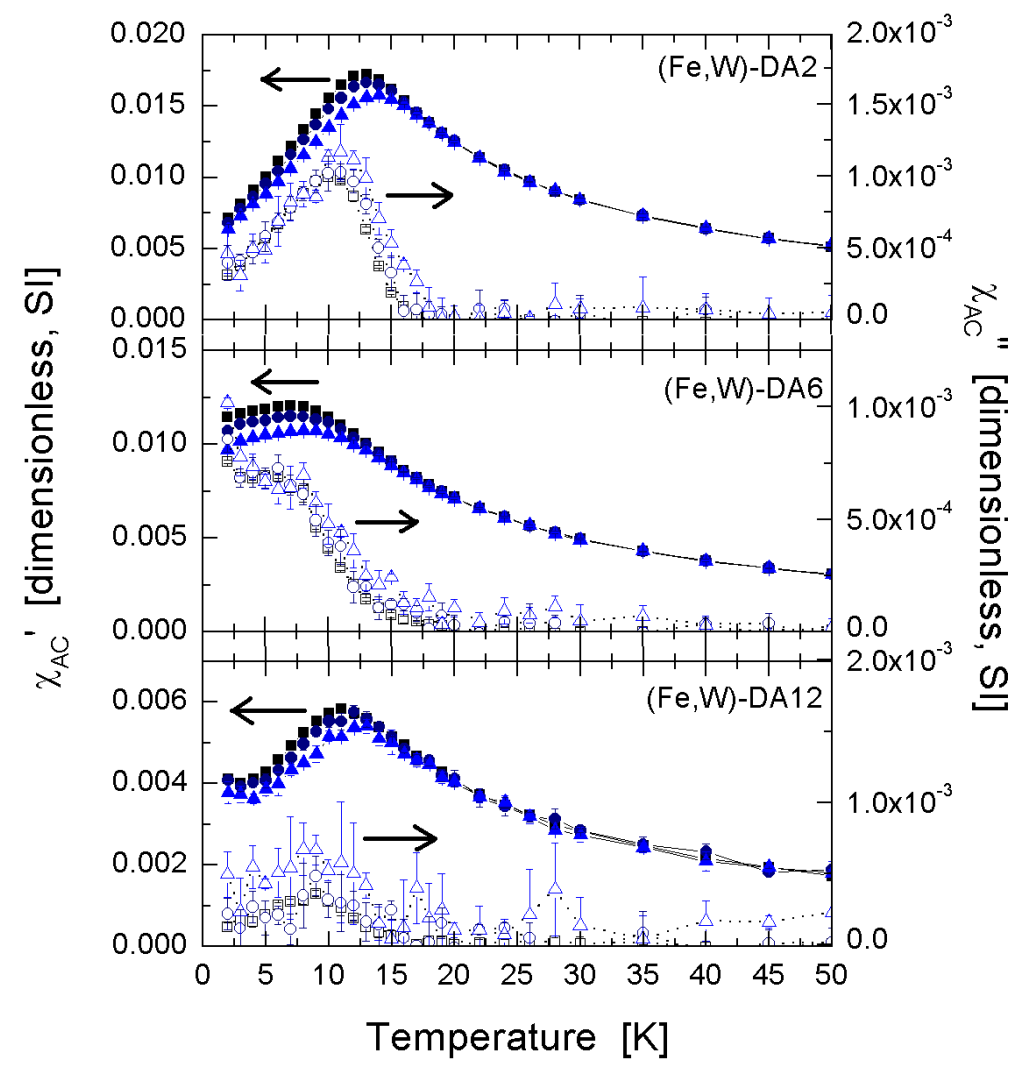

Figure 6.7: AC susceptibility for $(\mathrm{Fe}, \mathrm{W})$-DAn $(n=2,6,12)$, at frequencies of $33 \mathrm{~Hz}$ (squares), $333 \mathrm{~Hz}$ (circles) and $833 \mathrm{~Hz}$ (triangles). Closed symbols: real part $\left(\chi_{A C}^{\prime}\right)$, open symbols: imaginary part $\left(\chi_{A C}^{\prime \prime}\right)$. 
In the $\mathrm{AC}$ susceptibility of the same three samples (Figure 6.7), there is a significant frequency dependence of the peak feature present in both $\chi^{\prime}$ and $\chi^{\prime \prime}$ over quite a narrow frequency range. The relaxation time in these systems is estimated from these plots to be of the order of $1 \mathrm{~ms}$. The change in temperature at which the $\chi^{\prime}$ maximum occurs can be plotted against the frequency on a log scale. The quantity $\Delta T_{f} /\left[T_{f} \Delta\left(\log _{10} \omega\right)\right]$ (the slope of this graph) is often used as a measure of the frequency shift to compare with other known systems [208]. For the (Fe,W)-DAn hybrid materials, the following values are obtained:

$$
\begin{array}{lc}
\text { (Fe,W)-DA2 } & 0.064 \pm 0.011 \\
(\mathrm{Fe}, \mathrm{W})-\mathrm{DA} 6 & 0.127 \pm 0.023 \\
(\mathrm{Fe}, \mathrm{W})-\mathrm{DA} 12 & 0.084 \pm 0.012
\end{array}
$$

These values are similar to those reported for insulating spin glasses $(0.06-$ 0.28) [208].

A spin glass is the term used to describe a system in which the spins are disordered and only slowly fluctuating. Spin glasses can be observed in materials such as metal alloys (e.g. $\mathrm{Cu}_{1-x} \mathrm{Mn}_{x}, 0 \leq x \leq 0.1$ ) where a magnetic impurity is introduced in small amounts and is randomly distributed through the solid. In this case the interaction between spins is disordered. Spin glasses can also be observed in well-ordered systems where the direction of the spins is a random distribution (e.g. in HTS cuprates at low temperature and low hole concentrations [217]). In a metallic spin glass the magnetoresistance scales with the square of the magnetisation and the additional resistance arises from spin-flip scattering [142].

While a non-zero imaginary part $\left(\chi^{\prime \prime}\right)$ of the AC susceptibility normally indicates the presence of a ferromagnetic component (AC loss), the effect can be explained for the spin glass system by considering the mechanics of the measurement technique: an AC oscillating field is applied at a particular frequency $\omega$, and the resulting moment is measured. The measured moment also has frequency $\omega$, but because of the significant relaxation times (in the millisecond range) of spin glasses being comparable with the oscillation fre-

rather than susceptibilities. 


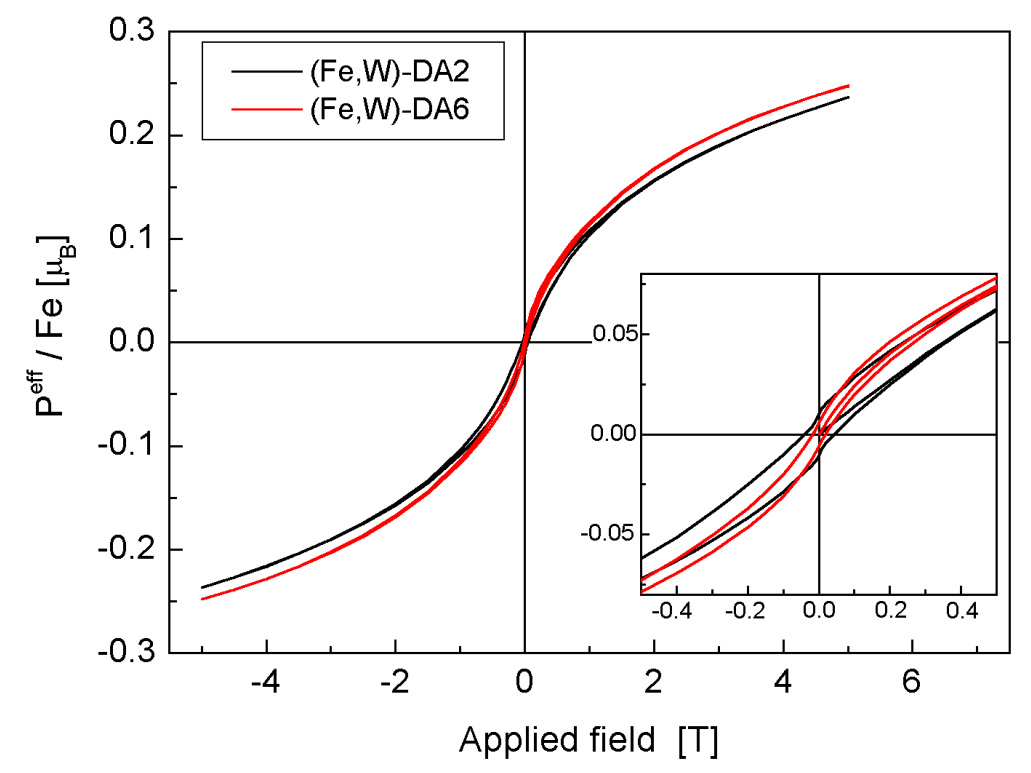

Figure 6.8: Hysteresis loops of (Fe,W)-DA2 (black) and (Fe,W)-DA6 (red). Insert: detail near $H=0$, showing the hysteretic behaviour.

quency, the response has a time lag. The resulting moment demonstrates a phase shift and an imaginary component is calculated. This is why as frequency decreases, the magnitude of the imaginary component also decreases, as one approaches the DC limit.

Field-dependent irreversibility in the DC susceptibility is also observed in spin glass systems [251]. Hence one would also expect to see hysteresis in $M(H)$ even though there is no long-range FM order, or FM component (from ferro- or ferrimagnetism or spin canting) in a spin glass.

Hysteresis loops performed on the DA2 and DA6 samples at $5 \mathrm{~K}$ are shown in Figure 6.8. These loops show hysteretic behaviour resembling that observed in other spin glass systems [251]. There is a gradual pinching off of the hysteresis at relatively high fields. The loops do not appear to be flattening towards a saturation magnetisation, and the absolute moments observed at much less than that expected for ferromagnetic $\mathrm{Fe}^{2+}\left(\mathrm{P}^{\text {eff }} / \mathrm{Fe}=\right.$ $4.90[9])$.

Magnetic hysteresis arises from the formation of domains (regions of FMaligned spins) within a material. In a spin glass there is no long-range order, 
so any 'domains' would not be strictly ferromagnetically aligned. However it is possible that there could be regions where the average spin is aligned in a different direction to the average spin in a neighbouring 'domain'. Domain boundaries normally pin to defects in the crystal or spin structure, and when the domain size equals or exceeds the grain size, there are no longer multiple domains and the hysteresis loops pinch off. In a spin glass where 'domains' might arise from an average spin direction, the domain would gradually enlarge to encompass the entire grain, causing the hysteresis loop to close. The width of the hysteresis loop is a measure of the anisotropy [251].

The irreversibility in the (Fe,W)-DAn hybrids (in the DC susceptibility) is suppressed at relatively low fields, indicating that the domain walls move rapidly in these materials. Of the five transition metal ion tungstate series, Fe appears to have the lowest crystallinity, as indicated from the quality of the XRD powder patterns, indicating small grain sizes, or disorder within the inorganic layer structure, or both.

It is known that manganese occurs in the present hybrid compounds as $\mathrm{Mn}^{2+}$ (from XANES, Section 5.2.5), and the $P^{e f f}$ values obtained from CurieWeiss fits agree well with the other transition metals also being present as $2+$ ions. However, $\mathrm{Fe}^{2+}$ is readily oxidised to $\mathrm{Fe}^{3+}$ which would cause a change in $P^{e f f}$ and hence the magnitude of the Curie constant. Valence fluctuations (such as $\mathrm{Fe}^{2+} \mathrm{W}^{6+} \mathrm{O}_{4} \rightarrow \mathrm{Fe}^{3+} \mathrm{W}^{5+} \mathrm{O}_{4}$ ) could account for this change in the DC susceptibility, and also for the frequency-dependence observed in the AC susceptibility.

Mössbauer measurements performed on (Fe,W)-DA2 and (Fe,W)-DA6 at $4.2 \mathrm{~K}$ revealed the presence of two Fe sites, each with a hyperfine magnetic field. Broad lines are observed, which indicates there is a distribution of magnetic fields on both sites. This could be caused by structural disorder or an artifact of the technique, as the timescale of the Mössbauer measurement is $\sim 10^{-7} \mathrm{~s}$, much shorter than the relaxation time of a spin glass. A static spin arrangement with FM ordering would be observed on the Mössbauer timescale. Therefore the hyperfine splitting would be observed, even though no magnetic order is present. To determine the presence of a spin glass, measurements need to be performed near the spin glass freezing temperature 
$T_{f}$, where the relaxation times are shorter. A single-line spectrum will be observed, corresponding to the disordered spins [208].

A comparison between the manganese and iron series suggests there is a large difference in the coupling between the magnetic ions in each case. The DC susceptibility of the manganese samples is virtually independent of field, and displays no irreversibility. This indicates there is strong coupling present, described by antiferromagnetic Heisenberg chains, in combination with larger grains and good crystallinity.

The iron samples on the other hand have very weak coupling, which is affected by relatively small fields. It is postulated that the iron tungstate hybrid system is an example of a spin glass. It is quite possible that the inorganic layer structure is disordered, rather than the well-ordered 'stripe' structure proposed for manganese, which could result in a spin glass and the observed magnetic phenomena in (Fe,W)-DAn.

\subsubsection{Co, Ni and $\mathrm{Cu}(\mathrm{M}, \mathrm{W})-\mathrm{DA} 2$}

As mentioned in Chapter 5, cobalt, nickel and copper tungstate hybrids with DA2 as the organic molecule have virtually the same structure as evidenced by XRD (accounting for the Jahn-Teller distortions in the copper system). It is conceivable then that one might expect these samples to have similar magnetic behaviour.

The DC susceptibility of the three samples is independent of field and exhibits no transitions in the range studied $(2-300 \mathrm{~K})$. The ZFC and FC curves are identical, indicating that there is no irreversibility. The DC susceptibility curves follow the Curie law exactly (with $\Theta=0$ within experimental error), indicating that the spins are non-interacting, resulting in pure localised moment paramagnetism with no phase transitions. A plot of $1 / \chi$ versus temperature is shown in Figure 6.9. This is a visual means of confirming the Curie-Weiss law - the plots are linear and pass through the origin.

The parameters from the Curie-Weiss fits are given in Table 6.2. The Weiss temperatures are all very similar and so close to zero that it could be said that the samples obey the Curie law $(\chi=C / T)$. The Curie constants 


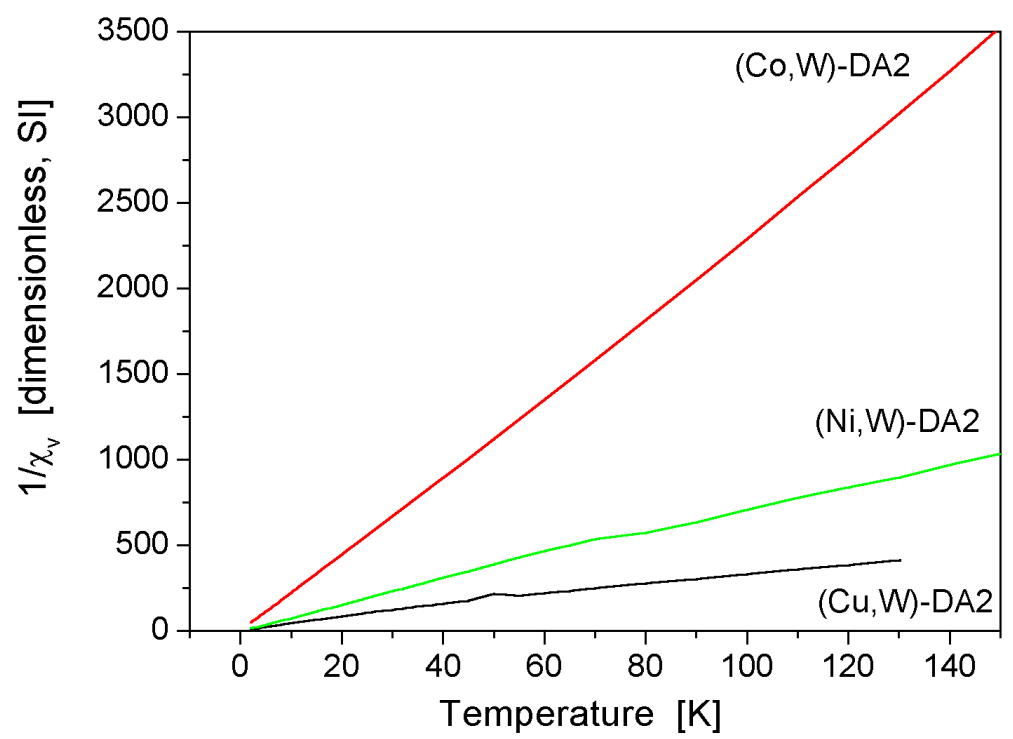

Figure 6.9: 1/ $\chi$ plot for $(\mathrm{M}, \mathrm{W})-\mathrm{DA} 2(\mathrm{M}=\mathrm{Co}, \mathrm{Ni}, \mathrm{Cu})$.

\begin{tabular}{|c|c|c|c|}
\hline & Experimental $P^{\text {eff }}\left[\mu_{B}\right]$ & Expected $P^{\text {eff }}\left[\mu_{B}\right]$ & $\Theta[\mathrm{K}]$ \\
\hline \hline$(\mathrm{Co}, \mathrm{W})-\mathrm{DA} 2$ & 3.85 & 3.87 & -0.23 \\
$(\mathrm{Ni}, \mathrm{W})-\mathrm{DA} 2$ & 2.90 & 2.83 & -0.15 \\
$(\mathrm{Cu}, \mathrm{W})-\mathrm{DA} 2$ & 1.90 & 1.73 & -0.28 \\
\hline
\end{tabular}

Table 6.2: Curie-Weiss fit parameters for $\mathrm{Co}, \mathrm{Ni}$ and $\mathrm{Cu}$ (M,W)-DA2 hybrid materials.

have been obtained from the slopes of the lines in Figure 6.9 and converted to $P^{e f f}$ values, using Equation 6.4. These agree extremely well with the expected $P^{e f f}$ values calculated from the spin of the ions ( $\frac{3}{2}$ for Co, 1 for $\mathrm{Ni}$, $\frac{1}{2}$ for copper).

These three samples represent a paramagnetic system with isolated magnetic moments, as evidenced by the almost zero Weiss temperature (no tendency towards AF or FM correlations), and the excellent agreement between the calculated and expected $P^{e f f}$ values. It is possible that the unknown structure of these hybrids is a 3-dimensional network or perhaps a cage structure, where the ions are separated by non-magnetic $\mathrm{WO}_{6}$ octahedra with very little coupling between them. 


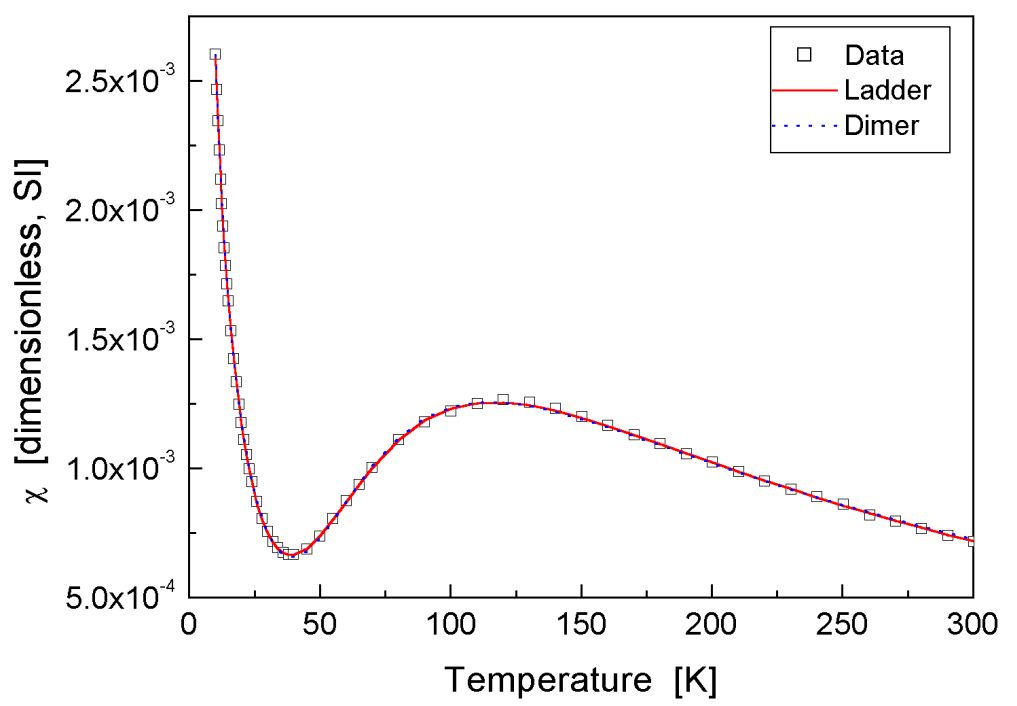

Figure 6.10: DC susceptibility of $(\mathrm{Cu}, \mathrm{W})$-DA6 with best fit curves for the spin dimer and spin ladder models.

\subsubsection{Copper tungstate hybrid}

The DC susceptibility of a $(\mathrm{Cu}, \mathrm{W})-\mathrm{DA} 6$ sample is shown in Figure 6.10. There is no irrevsibility and the susceptibility is independent of field. A broad local maximum is observed near $120 \mathrm{~K}$, which is not present in any of the other samples studied.

Divalent copper has a spin of only $\frac{1}{2}$, in contrast with manganese with spin $\frac{5}{2}$. This results in a much lower signal-to-noise ratio in the copper tungstate hybrids, requiring applied fields of $H>1000$ Oe in order to observe any definitive signal.

Reference [229] describes a $\mathrm{CuMoO}_{4}$-organic layered hybrid system in which the structure of the $\mathrm{CuMoO}_{4}$ inorganic layers varies depending on the shape of the organic ligand (n,m'-bipyridine $(\mathrm{n}, \mathrm{m}=3,4))$ and hence how it bonds to the inorganic layer. They performed single-crystal x-ray diffraction analysis on the three samples and observed layered structures with monomers, dimers, and tetramers of copper oxide species separated by non-magnetic molybdenum oxide tetrahedra. Following this they developed and adapted models to explain the $\chi(T)$ behaviour.

The dimer model (Equation 6.4.4) suggested in Ref. [229] is as follows: 


$$
\chi=\frac{2 N g^{2} \mu_{B}^{2}}{3 k_{B} T}\left[1+\frac{1}{3} \exp \left(-2 J / k_{B} T\right)\right]^{-1}+\frac{C}{T-\Theta}+T I P
$$

It fits the $(\mathrm{Cu}, \mathrm{W})$-DA6 data extremely well, with the following fit parameters:

$$
\begin{array}{r}
g=0.413 \pm 0.001 \\
J / k_{B}=-102.9 \pm 0.2 \mathrm{~K} \\
C=0.0277 \pm 0.0003 \mathrm{~K} \\
\Theta=0.17 \pm 0.08 \mathrm{~K} \\
T I P=-0.00022 \pm 0.000007 \\
\left(\chi^{2} / D o F=4.437 \times 10^{-11}\right)
\end{array}
$$

If the copper tungstate hybrid has the same inorganic structure as the manganese tungstate hybrids, a spin ladder may be formed. For this reason a spin ladder model was also fitted to the data. Due to the good agreement between the spin dimer model and the experimental data, we assume that in the spin ladder model (Section 6.4.1, page 140) the 'rung' exchange $\left(J_{1}\right)$ is much larger than the 'rail' exchange $\left(\mathrm{J}_{2}\right)$ and all other exchanges $\left(J_{\text {diag }}\right.$, $J_{\text {interchain }}$, etc.) are negligible. We used the fit in reference [126] for isolated chains with $J^{\prime} / J \geq 1\left(J^{\prime} \equiv J_{2} \equiv J_{\text {rung }}\right)$ :

$$
\begin{array}{r}
\chi(T)=\frac{N_{A} g^{2} \mu_{B}^{2}}{J^{\prime}} \chi *(t)+\frac{C}{T-\Theta}+T I P ; \\
\chi *(t)=\frac{\exp \left(-\Delta_{f i t}^{*} / t\right)}{4 t} \mathcal{P}_{q}^{p}(t) ; \\
t \equiv \frac{k_{B} T}{J^{\prime}}, \\
\mathcal{P}_{q}^{p}(t)=\frac{1+\sum_{n=1}^{p} N_{n} / T^{n}}{1+\sum_{n=1}^{q} D_{n} / t^{n}}
\end{array}
$$


where the coefficients $\Delta_{f i t}^{*}, N_{n}$ and $D_{n}$ are given in an appendix of ref. [126].

The fitting parameters are given as follows:

$$
\begin{array}{r}
g=1.920 \pm 0.008 \\
J_{1} / k_{B}=-0.3 \pm 1.4 \mathrm{~K} \\
J_{2} / k_{B}=213.6 \pm 1.1 \mathrm{~K} \\
C=0.0268 \pm 0.0003 \mathrm{~K} \\
\Theta=-0.0015 \pm 0.0004 \mathrm{~K} \\
T I P=-0.0003 \pm 0.00001 \\
\left(\chi^{2} / D_{o} F=2.3718 \times 10^{-11}\right)
\end{array}
$$

The two fits are compared in Figure 6.10 with the experimental data. The ladder model is shown as the solid red line and the dimer model as the dotted blue line. Comparing the two fits, we note firstly that the assumption $J_{2} \gg J_{1}$ is valid and therefore the spin dimer model should be sufficient to describe the $(\mathrm{Cu}, \mathrm{W})$-DA6 system. However, the spin dimer model does not give an accurate $g$ factor - the expected value is $\sim 2$, whereas the dimer model yields $g=0.413$. However the spin ladder model gives $g=1.920$, in good agreement with the expected value and consistent with other $\mathrm{Cu}^{2+}$ $S=\frac{1}{2}$ systems [229]. The exchange constant $J_{2}$ between the two $\mathrm{Cu}$ atoms of the dimer in the ladder model is roughly double that obtained for the dimer model; this is probably due to a difference of notation in the two models. In the exponential term of the dimer model (Equation 6.4.4), one has a factor of 2 that is not present in the exponential term of the spin ladder fit (Equation 6.4.4). The values of $C$ and TIP are virtually identical, and while $\Theta$ has different signs in each of the two models it has a small value so can be essentially treated as zero.

Structural analysis would assist greatly in determining the correct magnetic model to be used to analyse this compound. The fact that the spin ladder model gives a more reasonable value of $g$, and has a better $\chi^{2} / D o F$ value than the dimer model, indicates that the spin ladder model probably has some validity. 


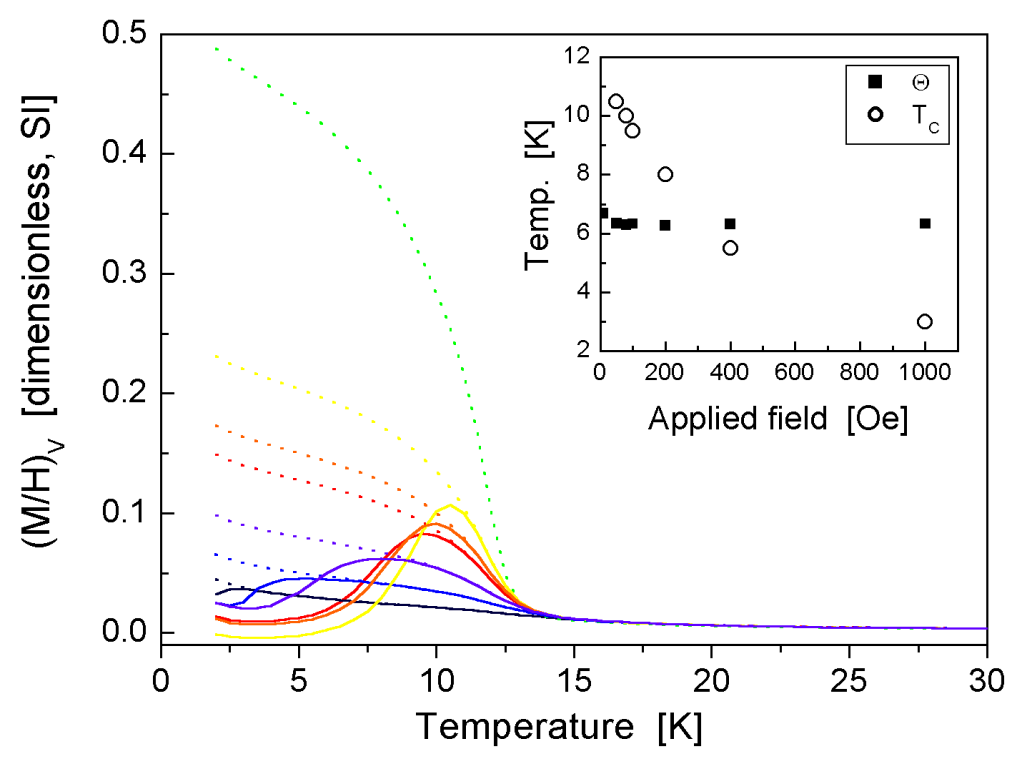

Figure 6.11: DC $M / H$ curves for $(\mathrm{Ni}, \mathrm{W})-\mathrm{DA} 6$ for applied fields of 10 Oe 1000 Oe. Insert: Curie-Weiss temperature $\Theta$ and $T_{C}=$ maximum of ZFC curves for the various fields studied.

\subsubsection{Nickel tungstate hybrid}

As shown in Table 6.1, the (Ni,W)-DA6 sample is one of the few to have a positive Weiss temperature, indicating the presence of ferromagnetic correlations. The DC $M / H$ curves of this sample also display a sharp ferromagnetic transition at around $12.5 \mathrm{~K}$, shown in Figure 6.11. As for the iron tungstate hybrids, the $M / H$ curves show significant field-dependence for relatively small applied fields, indicating either that the exchange is weak and easily disrupted by an external field or that the magnetic domains are quite small.

Despite the apparent ferromagnetic ordering, the absolute value of the saturation magnetisation of the sample is much less than that expected per $\mathrm{Ni}^{2+}$ ion for pure ferromagnetism, indicating that the sample is in fact either a ferrimagnet or canted antiferromagnet. Hysteresis loops were performed on this sample at temperatures from $2-15 \mathrm{~K}$ and fields from -6 to $+6 \mathrm{~T}$. A selection of these hysteresis loops is shown in figure 6.12. The hysteresis loops resemble a paramagnet at high fields plus a small region of irreversibility up to \pm 700 Oe at $5 \mathrm{~K}$. The linear part indicates that the phenomenon is not 


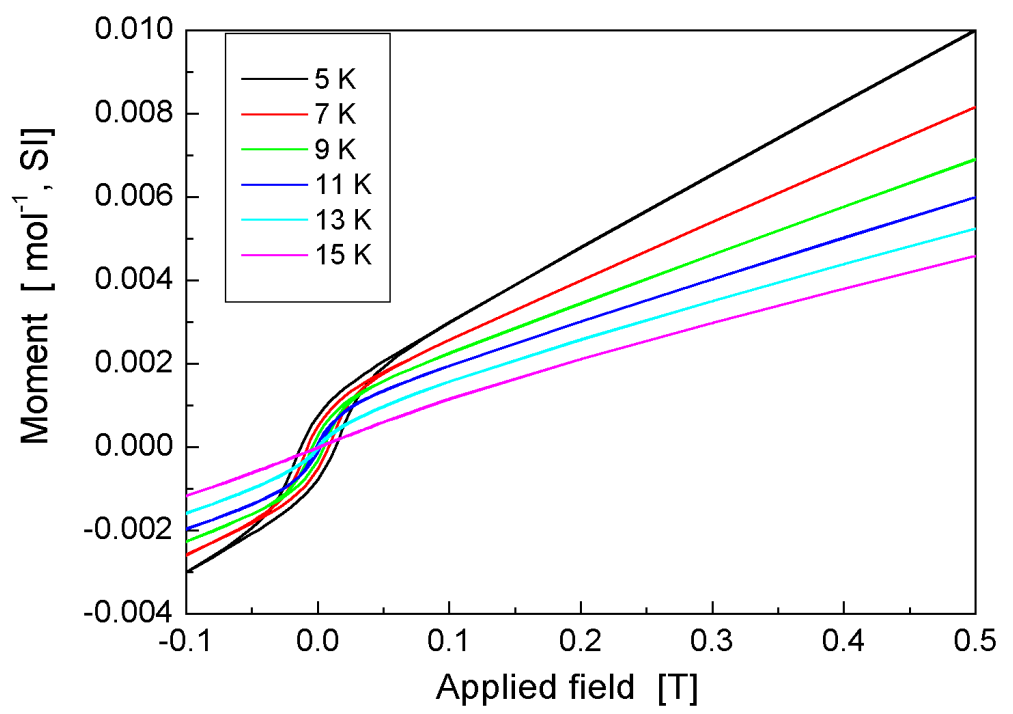

Figure 6.12: Hysteresis loops of $(\mathrm{Ni}, \mathrm{W})-\mathrm{DA} 6$ at various temperatures.

due to ferrimagnetism but rather to antiferromagnetism with spin canting. This is also more likely because only one magnetic species is expected $\left(\mathrm{Ni}^{2+}\right.$, $S=1$ ) and ferrimagnetism requires a regular arrangement of two different moments, ordered antiferromagnetically.

From analysis of hysteresis loops at different temperatures we can determine the canting angle [24]. At each temperature the non-compensated magnetisation was estimated by extrapolating the linear part of each curve to $H=0$. These values are plotted in Figure 6.13. Extrapolating this curve to $T=0$, we can obtain an estimate for the canting angle $\alpha$ of $0.46^{\circ}$, from the formula

$$
\tan \alpha=\frac{M_{n c}(T=0)}{g \mu_{B} S}
$$

(taking $S=1$ for $\mathrm{Ni}^{2+}$ ) [24].

The canting may result from distortion in the crystal structure. In Figure 6.13 we also notice the pinching-off of the curve at $15 \mathrm{~K}$, which is slightly above the apparent transition in the DC susceptibility.

A comparison of the $(\mathrm{Fe}, \mathrm{W})-\mathrm{DAn}$ series with $(\mathrm{Ni}, \mathrm{W})$-DA6 indicates that the behaviour of the two systems is quite different. The hysteresis loops of $(\mathrm{Fe}, \mathrm{W})-\mathrm{DAn}$ do not pinch off as rapidly as in (Ni,W)-DA6. (Ni,W)-DA6 


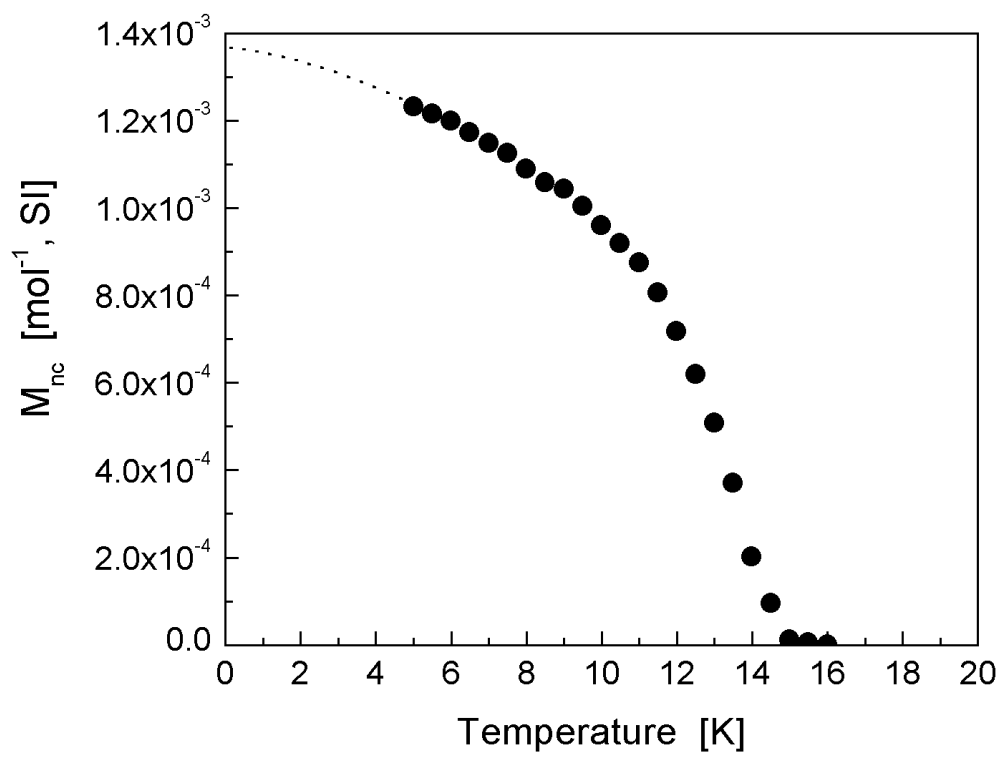

Figure 6.13: Non-compensated magnetisation $M_{n c}$ versus temperature for (Ni,W)-DA6, taken from the $H=0$ extrapolation of the high-field parts of the hysteresis loops in Figure 6.12.

displays no frequency-dependence in the AC susceptibility (Figure 6.14), and $\chi^{\prime}$ and $\chi^{\prime \prime}$ are quite similar in appearance. The apparent temperature of this transition as determined from the AC susceptibility and canting angle analysis is higher that that determined by the DC susceptibility, which is observed to occur in other spin-canted systems [284]. The sudden change in both the DC and AC susceptibilities of (Ni,W)-DA6 also indicates a definite magnetic phase transition.

\subsection{Summary}

Several examples of the variety of magnetic behaviour observed in the transition metal tungstate hybrids have been discussed. Without concrete crystal structures of any of the compounds it is difficult to provide models to compare with experiment. However we have been able to determine the basic magnetic characteristics of the materials:

The manganese tungstate hybrids have the same inorganic layer structure and very similar magnetic behaviour arising from this. In each case a fit to 


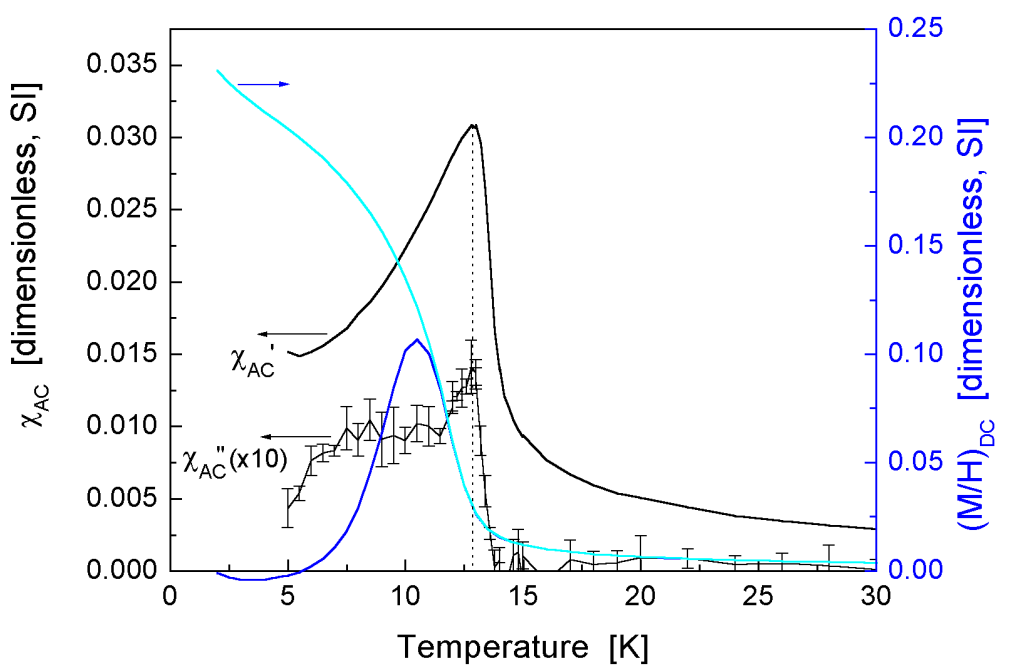

Figure 6.14: AC susceptibility of (Ni,W)-DA6, compared with the DC $M / H$ curve at 50 Oe.

the Curie-Weiss law at high temperatures results in a Weiss temperature of $\sim-20 \mathrm{~K}$. The high-temperature part of the curves can be fitted very well by an AF Heisenberg spin $\frac{5}{2}$ chain. There is a single antiferromagnetic transition which appears to be related to the interlayer distance, as the Néel temperature decreases with increasing interlayer spacing.

The iron tungstate hybrids exhibit characteristics in the DC and AC susceptibility and $M(H)$ hysteresis curves that are indicative of a spin glass, the most notable of these being the strong frequency dependence of the $\mathrm{AC}$ susceptibility. This may arise due to the apparent disorder in the inorganic structure as evidenced by XRD.

Cobalt, nickel and copper (M,W)-DA2 samples have the same inorganic structure according to XRD, and also have the same magnetic behaviour. They obey the Curie law, indicating that the magnetism in these materials arises from non-interacting free spins. From the slope of the $1 / \chi$ versus $T$ plot, we calculated effective moments per magnetic ion that agree extremely well with the predicted values, $P^{e f f}=g \sqrt{S(S+1)}$.

$(\mathrm{Cu}, \mathrm{W})-\mathrm{DA} 6$ displays a DC susceptibility curve that resembles a spin dimer system, but can be fitted equally well by a spin ladder model with very weak coupling along the length of the ladder. 
$(\mathrm{Ni}, \mathrm{W})-\mathrm{DA} 6$ is unusual among the hybrid materials because it displays a ferromagnetic-like transition at low temperatures. This FM behaviour is attributed to AF spin canting with a small canting angle of $\alpha=0.46^{\circ}$.

The strong field-dependence of some of the systems may result from their small grain sizes (which limits the magnetic domain size).

Examining the transition metal tungstate hybrids as a whole, we notice that the transition temperatures $T_{C}$ are all very similar $(10-15 \mathrm{~K})$, regardless of the spin of the transition metal ion. It is thought that $T_{C}$ is governed by $J_{\perp}$ and any weak anisotropy [222], which in turn will be affected by the interlayer spacing. This is especially observed to be the case in the (Mn,W)-DAn series, demonstrating how the physical properties can be tuned by altering the interlayer spacing of this low-dimensional structure. Thus the inherent low-dimensional nature of the hybrids is observed to affect the physical (namely, magnetic) properties of all the samples presented in this chapter. 


\section{Chapter 7}

\section{Doping}

Doping is a fundamental concept in semiconducting, metallic and strongly correlated electronic materials. The introduction of charge carriers (electrons and/or holes) causes changes to the electronic structure, shifts the Fermi level and may thus induce metallic conducting behaviour. The tungsten oxide-hybrid materials, as synthesised, are electrically insulating, and hence in order to use them effectively in a device, doping is necessary. In this chapter, possible experimental doping techniques are discussed and the results obtained for the tungsten oxide-based hybrid system and related materials are presented.

\subsection{Background}

In many solid state chemical systems such as the high-temperature superconducting cuprates or the colossal magnetoresistance manganites (described in Chapter 1), doping is easily achieved by controlling the stoichiometry of the starting materials. One can replace a fraction of a higher valence species by a lower valence species of roughly the same atomic radius (e.g. $\mathrm{Ca}^{2+}$ for $\mathrm{Y}^{3+}$ in $\mathrm{YBa}_{2} \mathrm{Cu}_{3} \mathrm{O}_{7}$ ) to induce hole-doping, or vice-versa to induce electrondoping [118]. Superconductivity is observed in HTS cuprates in the holedoped regime at doping levels of 0.06-0.25 [236], while other systems such as the CMR manganites exhibit a rich and varied phase behaviour across the entire doping range $0 \leq x \leq 1$, for $\mathrm{La}_{1-x} \mathrm{Ca}_{x} \mathrm{MnO}_{3}$ [237]. 
Silicon technology is also reliant on doping via replacing a certain proportion of silicon atoms by an aliovalent species, normally boron (hole-doping, positive carriers, 'p-type') or phosphorous (electron-doping, negative carriers, 'n-type'). For example, boron doping levels of only $10^{-5}$ are required to increase the conductivity of silicon by a factor of $10^{3}$ [138, p. 221]. Such doping in silicon effectively introduces defects in the crystal structure, which causes changes in the band structure near the Fermi level, allowing increased conduction to occur.

The tungsten bronze system, already discussed at length in Section 1.3, is another example of a solid state doped system exhibiting a wide solid solution range. Intercalation of a fraction of alkali metal ions with $1+$ charge causes the tungsten ions to reduce from $6+$ to $5+$, resulting in electrondoping of the system. As mentioned earlier these systems exhibit drastic colour changes and their electrical properties change from semiconducting to metallic. Similar effects are observed when oxygen is removed from the system, again reducing $\mathrm{W}^{6+}$ to $\mathrm{W}^{5+}$.

A similar experiment was attempted by adding selected transition metal ion solutions in the synthesis of the tungsten oxide hybrids (Chapter 5), however it was found that regardless of the stoichiometry of the starting materials, a product with 1:1 ratio of transition metal to tungsten was formed. X-ray absorption near-edge spectroscopy (XANES) studies show that the metal ions are present as $2+$ species and the tungsten as $6+$. Instead of doping, the transition metal hybrids form a completely different structure that is interesting in its own right.

Attempts were made to substitute $\mathrm{W}^{6+}$ by $\mathrm{Re}^{5+}, \mathrm{Ru}^{4+}$ and $\mathrm{Ta}^{5+}$ with no apparent success. We further investigated the possible intercalation of small monovalent metal ions. Addition of solutions of such metal ions (e.g. $\mathrm{Li}^{+}$, $\mathrm{Na}^{+}$) in the course of synthesis produced samples identical to the tungstenonly synthesis, with no evidence that the extra ions had been incorporated. Hence a more effective method of including extra ions is required.

Ion bombardment is a possible way to implant small metal ions, and is commonly used in the silicon industry. However for some materials, there are more cost-effective ways of achieving doping for commercial applications. 
For the organic-inorganic hybrids, there is also the danger of destroying the organic molecules due to the high kinetic energy of the incoming atoms, thus causing irreversible structural changes.

Another method of inducing doping in such a system is through fabrication of thin-film field-effect devices. These devices essentially use the thinfilm material as the doping component in a thin-film transistor. A conducting substrate acts as a gate electrode, next an insulating layer (e.g. $\mathrm{SiO}_{2}$ ), then a film of the desired material is deposited, and finally source and drain electrodes are deposited to complete the arrangement. An electric field is generated within the insulating layer by applying a DC voltage between the gate and source electrodes. This injects either electrons or holes, depending on the sign of the field, into the thin film under study. The current-voltage characteristics of the thin film material are then examined using the source and drain electrodes. This method has been successfully used with the metal halide hybrids [130, 129, 199], and we have had some preliminary success in fabricating and testing field-effect devices using the tungsten oxide hydrate. Similar efforts with the hybrid materials have not yet been successful, but this is nonetheless worth pursuing as it is not only as a means of doping the material but also for potential applications.

One relatively simple laboratory technique to set up and execute, using readily available equipment, is insertion of ions using an electrochemical cell. Another major benefit of the technique is that the reaction rate and doping level can in principle be well-controlled, through controlling the applied potential difference and the ramp rate. In this the ionic intercalant, and the resultant electronic carrier concentration, can be titrated with precision.

Two electrochemical doping techniques were explored: cyclovoltammetry, and constant potential electrochemistry.

Cyclovoltammetry is particularly useful for identifying redox processes, which give insight into the kinetics of oxidation or reduction. In a cyclovoltammetry experiment, the potential is swept several times at a constant rate over a certain voltage range while the current is measured. The resulting $\mathrm{I}-\mathrm{V}$ curves are loops consisting of a cathodic and anodic branch. The 
cathodic branch is measured as the potential is driven in the negative direction and reductive processes will be observed in the form of inverted peak features. The anodic branch is measured when the potential is swept in the positive direction, and oxidation processes will be observed. If the potential range being swept is wide enough, each reduction peak feature will have an associated oxidation peak feature. These are called redox couples and the absolute potential values of these, relative to the reference electrode, and the potential difference between them, give insight into the kinetics of each redox process.

Once the potentials for the desired oxidative or reductive reaction have been determined with cyclovoltammetry, one can perform constant potential electrochemistry to alter the oxidation state of the system. In a constant potential experiment, the voltage is fixed at a static value, and the current measured as a function of time to ensure that the system has come to equilibrium. The sample retains its new oxidation state when is removed from the solution, even though this may be short-lived.

\subsection{Previous studies}

Because of the ability of tungsten trioxide to incorporate small alkali metal ions, it has been extensively investigated as a possible material for electrochromic applications (see Section 1.3). Alkali metal ions can be incorporated into $\mathrm{WO}_{3}$ in the past by solid state synthesis $[33,14]$, ion bombardment [19], or via electrochemistry. More detail of the latter is as follows.

$\mathrm{WO}_{3}$ films are deposited by sol-gel methods from tungsten peroxide solutions [212, 164], by electron-beam deposition [164], or by RF-sputtering [132]. They may then be annealed to improve the crystallinity.

$\mathrm{H}_{2} \mathrm{SO}_{4}$ was used as an electrolyte solution in Ref. [132] to electrochemically intercalate hydrogen ions into amorphous and crystalline $\mathrm{WO}_{3}$ films. The transmittance of the films was observed to decrease from $80 \%$ to $30 \%$ upon colouration.

Lithium is another common electrochemical intercalate for both $\mathrm{WO}_{3}$ and $\mathrm{MoO}_{3}$ films $[212,260]$. It displays a characteristic redox curve as illustrated 
in the experimental results presented later in this thesis. In addition, $\mathrm{WO}_{3}$ can also be intercalated with $\mathrm{H}^{+}, \mathrm{Li}^{+}$, and $\mathrm{Na}^{+}$using an appropriate solid state electrolyte $[67,101]$.

Hydrated tungsten and molybdenum oxides have also been studied via electrochemical intercalation of lithium [108, 127, 128, 164]. These are observed to be less stable over many cycles than $\mathrm{WO}_{3}$ films, due to the lithium ions replacing the hydrated protons [164]. Both $\mathrm{Li}^{+}$and $\mathrm{H}^{+}$are more mobile in the layered hydrate structure than $\mathrm{WO}_{3}$, allowing them to leach out over time [127].

In this work, we examine the possibility of intercalating $\mathrm{Na}^{+}$(using aqueous $\mathrm{NaCl}$ as the electrolyte) into $\mathrm{WO}_{3}$ and $\mathrm{H}_{2} \mathrm{WO}_{4}$ particulate films via electrochemistry in more detail. Most previous studies focused on the insertion of $\mathrm{Li}^{+}$using $\mathrm{LiClO}_{4}$ in propylene carbonate, a non-aqueous electrolyte. To the best of our knowledge, the equivalent insertion of $\mathrm{Na}^{+}$(either in an aqueous or non-aqueous electrolyte) has not yet been reported. The present studies on $\mathrm{WO}_{3}$ and $\mathrm{H}_{2} \mathrm{WO}_{4}$ form the basis for comparing the hybrid films with a known reference material.

\subsection{Electrochemical Doping}

We have investigated electrochemical doping of $\mathrm{WO}_{3}, \mathrm{H}_{2} \mathrm{WO}_{4}$ and the hybrid materials W-DA2 and W-DA6, as described in Section 2.3. This should enable us to compare the results for $\mathrm{WO}_{3}$ and $\mathrm{H}_{2} \mathrm{WO}_{4}$ with those for different ionic species and electrolytes in literature, and then use these as base systems with which to compare the two hybrid materials.

\subsection{1 $\quad \mathrm{WO}_{3}$}

The redox curve of $\mathrm{WO}_{3}$ is shown in Figure 7.1. It is qualitatively identical to the redox curves reported for $\mathrm{WO}_{3}$ thin films prepared by various techniques, with intercalated $\mathrm{H}^{+}$and $\mathrm{Li}^{+}$species from various electrolyte solutions $[26$, 40, 127, 212].

A scan rate of $20 \mathrm{mV} / \mathrm{s}$ was chosen for the experiments, as faster scan rates (e.g. $50 \mathrm{mV} / \mathrm{s}$ ) cause the curves to flatten out somewhat and the area 


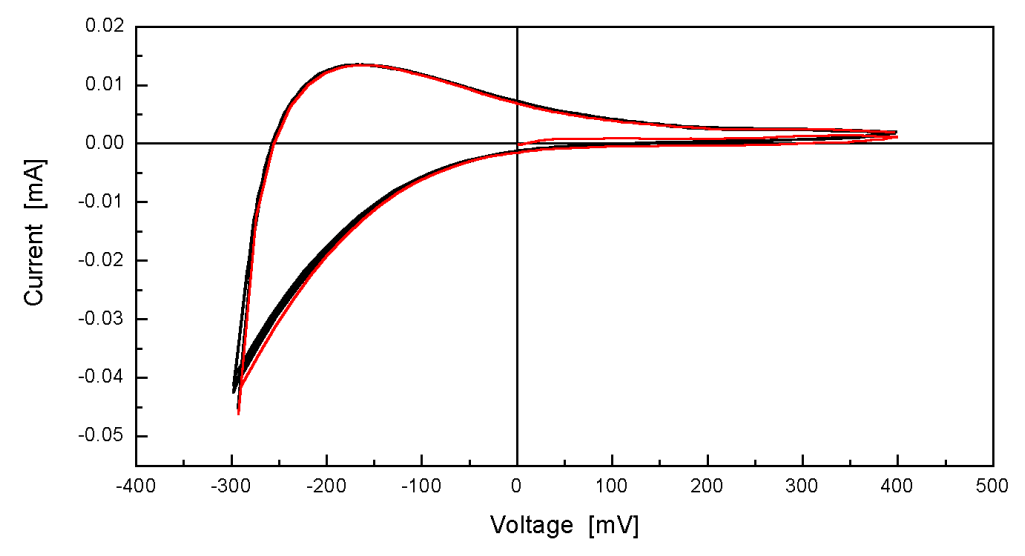

Figure 7.1: Cyclic voltammetry curves of $\mathrm{WO}_{3}$ with aqueous $\mathrm{Na}^{+}$. The red curve shows the initial loop.

enclosed by the curves decreases. This corresponds to a chemical equilibrium imbalance, indicating the presence of a limiting factor on the mobility of $\mathrm{Na}^{+}$ ions within the $\mathrm{WO}_{3}$ structure.

As the potential tends towards more negative values in each cycle, an increasing cathodic current is observed, along with a corresponding blue colouration of the sample. Along this part of the cycle $\mathrm{Na}^{+}$is being incorporated within the $\mathrm{WO}_{3}$ structure, corresponding to reduction according to the formula:

$$
\mathrm{WO}_{3}+\mathrm{xNa}^{+}+\mathrm{xe}^{-} \rightarrow \mathrm{Na}_{\mathrm{x}} \mathrm{WO}_{3}
$$

As the ramping direction changes and the applied voltage tends towards positive values, the current is observed to pass through a broad maximum on the anodic (positive) side. This is the oxidation peak, corresponding to the outflow of electrons as $\mathrm{Na}^{+}$is removed from the structure. As a result the blue colouration is observed to fade continuously up to the maximum positive voltage, where the cycle begins again. The area within the loop, $\int I d V$, represents the dissipation per cycle associated with work done against the lattice and the associated change in unit cell volume.

It was observed that the first cycle evidences completely reversible colouration from yellow to blue and back, however in later cycles the blue colour remains even after cycling to the maximum positive voltage. This could be 


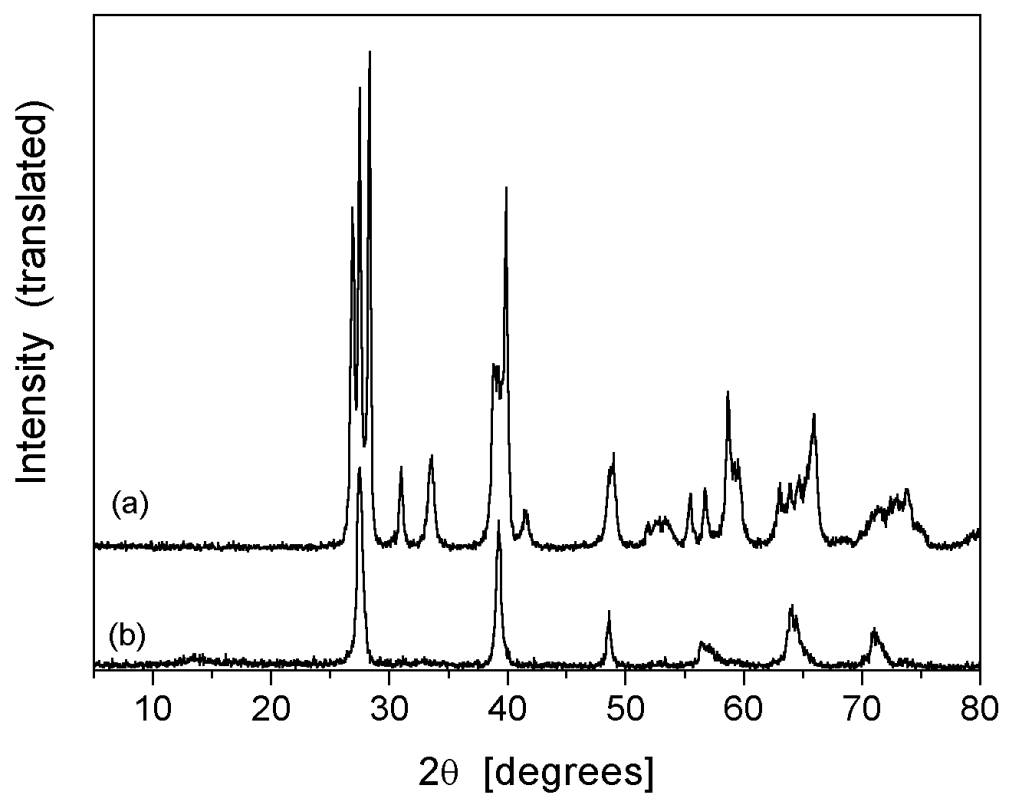

Figure 7.2: XRD patterns of (a) triclinic $\mathrm{WO}_{3}$ and (b) cubic $\mathrm{Na}_{x} \mathrm{WO}_{3}$ obtained after electrochemical intercalation.

due to the degrading effect of water on the $\mathrm{WO}_{3}$ structure [164]. Another contributing factor could be the formation of a stable phase of $\mathrm{Na}_{x} \mathrm{WO}_{3}$, from which a significant fraction of the $\mathrm{Na}^{+}$ions are not dislodged during the anodic (oxidation) leg.

XRD patterns of $\mathrm{WO}_{3}$ and an electrochemically doped sample are shown in Figure 7.2. The triclinic cell of $\mathrm{WO}_{3}$ converts to the cubic cell of $\mathrm{Na}_{x} \mathrm{WO}_{3}$. From the $d$-spacing obtained from the XRD pattern, $(3.767 \AA)$ we can estimate the doping level $x$ in $\mathrm{Na}_{x} \mathrm{WO}_{3}$ to be around 0.1 , by comparing this $d$-spacing value to literature results (as detailed in Section 4.4).

\subsection{2 $\quad \mathrm{H}_{2} \mathrm{WO}_{4}$}

A cyclic voltammogram of $\mathrm{H}_{2} \mathrm{WO}_{4}$ with aqueous $\mathrm{NaCl}$ as the electrolyte is shown in Figure 7.3. The shape of the curve is very similar to that of $\mathrm{WO}_{3}$ (Figure 7.1), as observed in literature also [128].

However, unlike the $\mathrm{WO}_{3}$ curve, there is some irreversibility evident from the steadily reducing cathodic current at negative voltages. A visual inspection while the experiment was underway provided additional confirmation 


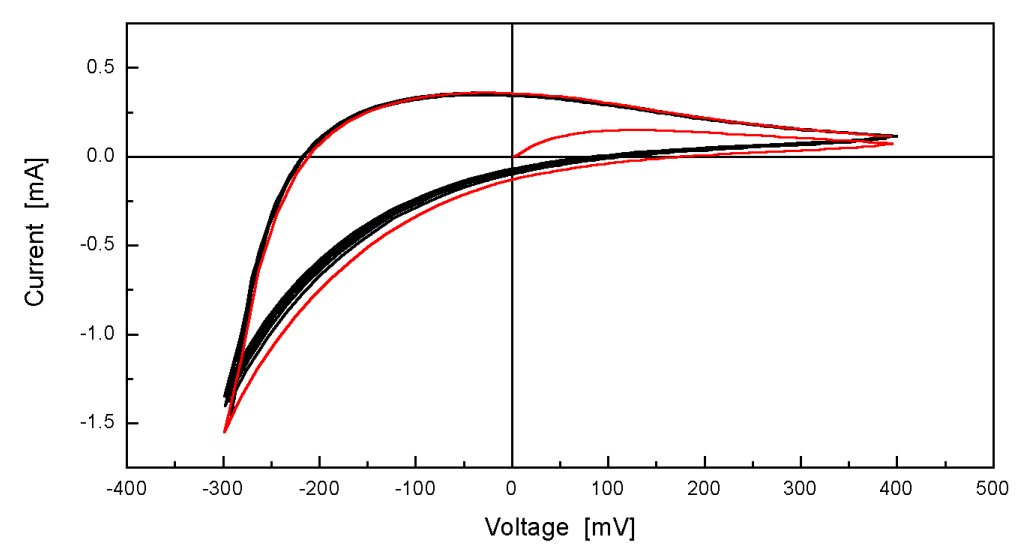

Figure 7.3: Cyclic voltammetry curves of $\mathrm{H}_{2} \mathrm{WO}_{4}$ with aqueous $\mathrm{Na}^{+}$. The red curve shows the initial loop.

that the cycle is irreversible, as the sample did not regain its original yellow colour during the anodic leg of the cycle. This irreversibility is described in literature and attributed to the substitution of $\mathrm{H}$ (on terminal $\mathrm{W}-\mathrm{OH}_{2}$ groups) by the intercalated ion. The released $\mathrm{H}$ then participates in the subsequent redox cycles, but its colouration is much less efficient than the substituted ion [164].

The magnitude of the current is much larger than in $\mathrm{WO}_{3}$, suggesting that intercalation of $\mathrm{Na}^{+}$is easier within $\mathrm{H}_{2} \mathrm{WO}_{4}$ than $\mathrm{WO}_{3}$. However this can be affected by a number of factors unrelated to electronic or ionic mobility, such as the material:binder ratio, grain size and quality of adhesion to the gold electrode.

XRD patterns of three $\mathrm{H}_{2} \mathrm{WO}_{4}$ samples electrochemically intercalated with $\mathrm{Li}^{+}, \mathrm{Na}^{+}$and $\mathrm{K}^{+}$from their respective aqueous chloride solutions are compared with $\mathrm{H}_{2} \mathrm{WO}_{4}$ in Figure 7.4. The corresponding cell parameters are given in Table 7.1 for an orthorhombic cell.

As shown in Table 7.1, both Li and Na cause similar increases in the cell volume of $\mathrm{H}_{2} \mathrm{WO}_{4}$ (although the amount of each is unknown, and the ions themselves are of different sizes). $\mathrm{K}$ does not appear to change the volume much, which in view of its significantly larger ionic radius, implies that intercalation was not as successful as for Li and Na. K may be too bulky to intercalate within the structure to the same doping levels as the smaller ions 


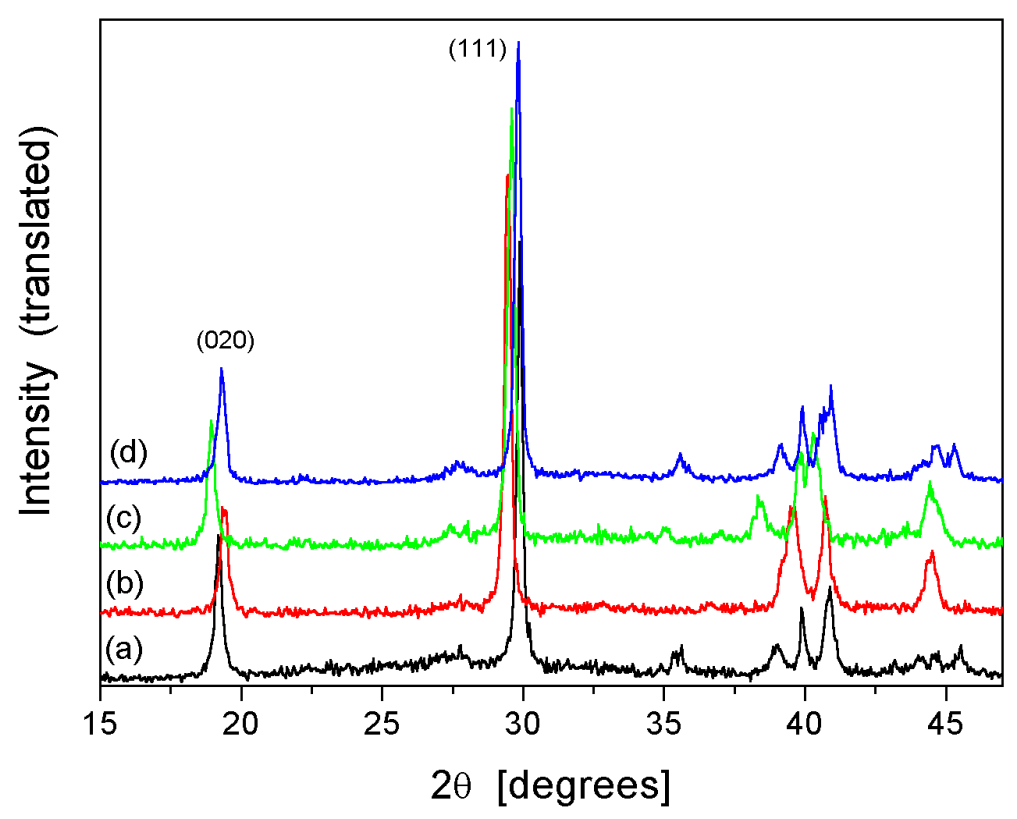

Figure 7.4: XRD patterns of (a) $\mathrm{H}_{2} \mathrm{WO}_{4}$, and intercalated with (b) $\mathrm{Li}$, (c) $\mathrm{Na}$, (d) K.

\begin{tabular}{|c|ccc|c|}
\hline Sample & \multicolumn{3}{|c|}{ Cell parameters } & Volume \\
& a $[\AA]$ & b $[\AA]$ & c $[\AA]$ & {$\left[\AA^{3}\right]$} \\
\hline \hline $\mathrm{H}_{2} \mathrm{WO}_{4}$ & 5.249 & 10.711 & 5.133 & 288.588 \\
$\mathrm{Li}_{x} \mathrm{H}_{2} \mathrm{WO}_{4}$ & 5.263 & 10.622 & 5.281 & 295.540 \\
$\mathrm{Na}_{x} \mathrm{H}_{2} \mathrm{WO}_{4}$ & 5.249 & 10.870 & 5.221 & 297.906 \\
$\mathrm{~K}_{x} \mathrm{H}_{2} \mathrm{WO}_{4}$ & 5.241 & 10.684 & 5.158 & 288.830 \\
\hline
\end{tabular}

Table 7.1: Cell parameters of electrochemically intercalated $\mathrm{H}_{2} \mathrm{WO}_{4}$. 


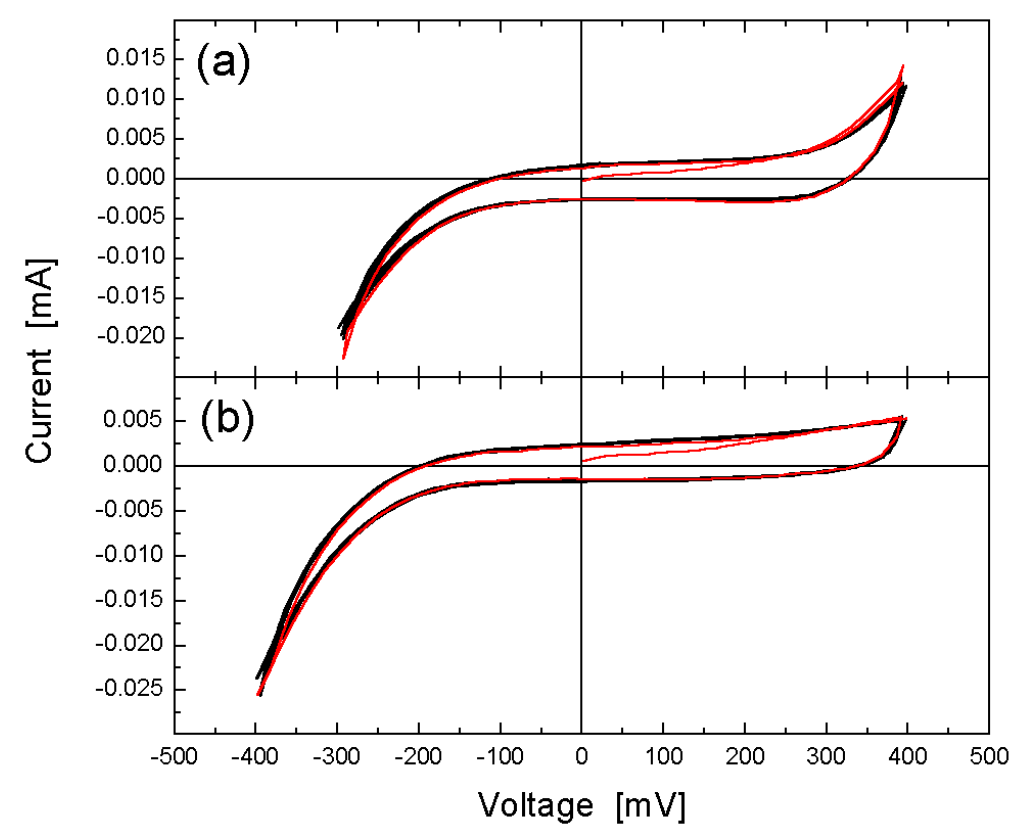

Figure 7.5: Cyclic voltammetry curves of (a) W-DA2 and (b) W-DA6 with aqueous $\mathrm{Na}^{+}$. The red curves show the initial loop.

(a similar result was obtained computationally and experimentally for potassium tungsten bronzes, see Chapter 4). By visual inspection, the colouration observed is also less, as the blue colour is not as intense as for the Li- and Na-intercalated samples.

\subsubsection{W-DA2 and W-DA6 hybrids}

Cyclic voltammograms of W-DA2 and W-DA6 hybrids, with aqueous $\mathrm{NaCl}$ as the electrolyte, are shown in Figure 7.5. The curves are qualitatively similar to each other, displaying no peaks or other features related to redox processes. The difference between the cathodic and anodic legs is small, as are the absolute magnitude of the currents, indicating that no intercalation is taking place. In addition there is no change in the colouration observed visually, and XRD displays no lattice expansion to indicate the incorporation of additional ions.

There are a number of possible reasons for the lack of any electrochemical effect: 


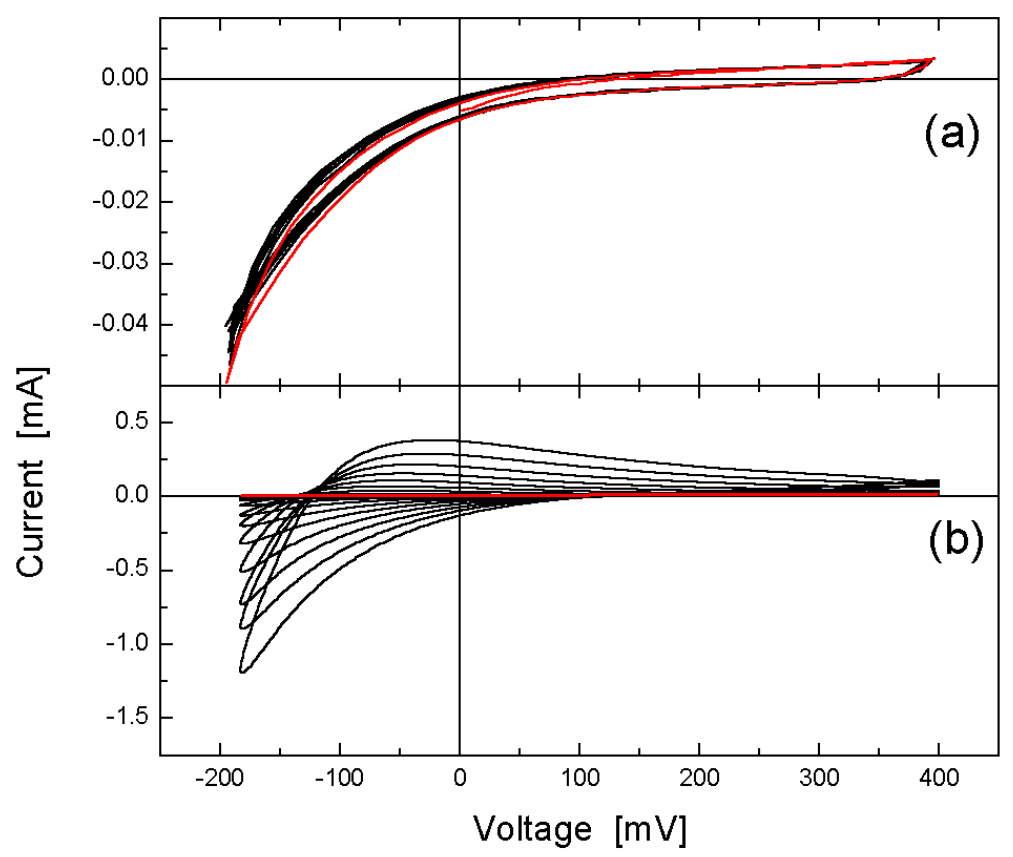

Figure 7.6: Cyclic voltammetry curves of (a) W-DA2 and (b) W-DA6 in 0.5 $\mathrm{M}$ aqueous $\mathrm{H}_{2} \mathrm{SO}_{4}$. The red curve shows the initial loop.

- It is thought that the terminal amine group has occupied all the possible sites for $\mathrm{Na}^{+}$, so for $\mathrm{Na}^{+}$to be intercalated would require significant disruption of the organic molecules.

- The presence of the amine molecule within the hybrid structure is very stable, and cannot be replaced by $\mathrm{Na}^{+}$. This could also explain why we were unable to form hybrids including $\mathrm{Na}^{+}$via the solution-based synthesis technique.

While this may appear to be a negative result, it is further testimony to the stability of the W-DAn structure. This was further tested by additional electrochemical experiments using aqueous $\mathrm{H}_{2} \mathrm{SO}_{4}$ to investigate the stability of the hybrids in an acidic medium. The cyclic voltammograms of the two samples are shown in Figure 7.6.

Figure 7.6a, showing the results for W-DA2, indicates that no reaction takes place. There is no obvious change in the shape of the curve over many cycles. This indicates that W-DA2 is chemically stable in an acidic medium. 
On the other hand, cyclic voltammograms of W-DA6 (Figure 7.6b) show a rapid progression from the characteristic W-DA6 curve (Figure 7.5b) to become more like those of $\mathrm{H}_{2} \mathrm{WO}_{4}$ (Figure 7.3). XRD of the product obtained at the end of this experiment indicated that $\mathrm{WO}_{3} \cdot n \mathrm{H}_{2} \mathrm{O}$ had been formed, thus the DA6 organic had been leached out. This was also observed in a separate experiment, where W-DA6 was placed in an aqueous solution of $\mathrm{HCl}\left(1 \mathrm{molL}^{-1}\right)$ overnight. The $\mathrm{W}$-DA6 powder developed the characteristic yellow colour of $\mathrm{WO}_{3} \cdot 2 \mathrm{H}_{2} \mathrm{O}$ and XRD confirmed the complete conversion of W-DA6 to $\mathrm{WO}_{3} \cdot 2 \mathrm{H}_{2} \mathrm{O}$.

Anomalies in the behaviour of W-DA2 to W-DA6 (and other W-DAn hybrids) were also observed in TGA and IR (Sections 3.2.4 and 3.3 respectively), namely that DA2 appears not to adopt the protonated ammonium form in the hybrid, whereas the other DAn molecules do. This indicates that a different bonding mechanism may be active in the W-DA2 sample, which appears to be more resistant to temperature (from the higher decomposition temperature in TGA) and chemical attack (from the acid studies here) than the other W-DAn materials.

A possible chemical reaction for acid leaching of the W-DAn hybrids (using $\mathrm{HCl}$ as an example) is

$$
\mathrm{WO}_{4}{ }^{2-} \cdot \mathrm{DAnH}_{2}{ }^{2+}+2 \mathrm{H}^{+} \mathrm{Cl}^{-}+\mathrm{nH}_{2} \mathrm{O} \rightarrow \mathrm{WO}_{4} \cdot(\mathrm{n}+1) \mathrm{H}_{2} \mathrm{O}+\mathrm{DAn} \cdot 2 \mathrm{HCl}
$$

i.e. a chloride salt of the DAn molecule is formed. However, for W-DA2 the amines are not protonated so therefore they do not react with the $\mathrm{Cl}^{-}$ions to form a salt.

The lack of any redox features in the voltammetry curves indicates that not only is the $\mathrm{WO}_{4} \cdots \mathrm{DA} 2$ bond not affected, but there is also no intercalation of $\mathrm{H}^{+}$within the structure to protonate the terminal $-\mathrm{NH}_{2}$ groups to $-\mathrm{NH}_{3}{ }^{+}$in W-DA2.

Preliminary electrochemical doping experiments were also performed on (Mn,W)-DA2 and (Mn,W)-DA6 samples. These also exhibit very small currents in the I-V curves, like their tungsten hybrid counterparts, however there were indications of a redox couple in the region from -200 to $+200 \mathrm{mV}$. This 
may correspond to the successful intercalation and deintercalation of $\mathrm{Na}^{+}$ ions within the manganese tungstate hybrid structure. In Section 5.2.5 a structure was proposed for these materials based on an inorganic layer of alternating Mn and W 'stripes', with the organic molecules interacting with the Mn sites. Such a structure may exhibit vacant sites between the apical oxygens of the tungsten atoms where sodium could be intercalated. As for $\mathrm{WO}_{3}$ and $\mathrm{H}_{2} \mathrm{WO}_{4}$, intercalation of $\mathrm{Na}^{+}$would result in the reduction of tungsten from $\mathrm{W}^{6+}$ to $\mathrm{W}^{5+}$.

While no difference in structure or colour of the manganese tungstate samples was observed after electrochemical treatment, changes in the magnetisation at low temperatures was observed in samples held for a period of time at a positive potential $(+400 \mathrm{mV})$. This may be due to disruption of the magnetic structure in the AF-ordered state at $T<T_{N}$. These preliminary results suggest that this is a promising area for future work.

\subsection{Summary}

Comparing the electrochemical doping results for $\mathrm{WO}_{3}$ and $\mathrm{H}_{2} \mathrm{WO}_{4}$, we observe several differences in the behaviour in the 2-dimensional as opposed to the 3-dimensional system. $\mathrm{WO}_{3}$ doping with $\mathrm{Na}^{+}$is much more ratedependent than $\mathrm{H}_{2} \mathrm{WO}_{4}$, indicating that the diffusion limits of $\mathrm{WO}_{3}$ are lower (i.e. more difficult for $\mathrm{Na}^{+}$to migrate within the 3-dimensional structure). The increased mobility of $\mathrm{Na}^{+}$in $\mathrm{H}_{2} \mathrm{WO}_{4}$ is further seen in the time dependence of the colouration, where $\mathrm{Na}^{+}$is observed to leach out over time [127].

In the tungsten oxide hybrids we observe no significant electrochemical effect when aqueous $\mathrm{Na}^{+}$was used as the electrolyte. This indicates that the possible intercalation sites for $\mathrm{Na}^{+}$are occupied. However there was an indication in preliminary experiments in the manganese tungstate hybrid equivalents that such sites may exist and a limited amount of $\mathrm{Na}^{+}$is presumed to have been incorporated because of the observation of a redox couple in the cyclovoltammograms.

A bilayer compound, $\mathrm{W}_{2} \mathrm{O}_{7}$-DAn, if synthesised, would be an interesting 
material of which to examine the electrochemical behaviour. Such a compound combines the structures of $\mathrm{WO}_{3}$ (between the two linked $\mathrm{WO}_{4}$ layers) and the W-DAn hybrid materials. There are many other such possibilities for doping these materials to alter their physical properties. 


\section{Chapter 8}

\section{Outlook and Conclusion}

Throughout this thesis a number of research areas have been identified which would benefit from further in-depth study. This includes possible new materials and additional experiments which could be performed on promising materials already synthesised. Here, the possibilities are organised concerning tungsten oxide hybrids, transition metal hybrids, magnetisation studies, doping, and soft-mode behaviour, before providing the conclusion of the work.

\subsection{Tungsten oxide hybrids}

So far only one chemical variant of the tungsten oxide-organic hybrid system has been explored, that of a monolayer $\mathrm{WO}_{4}$ unit separated by organic amines. Both the inorganic and the organic components can be altered.

In the metal halide-amine hybrid systems, hybrids with multiple inorganic layers (bilayers, trilayers etc.) were synthesised by simply altering the stoichiometries of the starting materials [196, 200]. We attempted this with the tungsten oxide system, however multiple layers were not formed, and in fact it was found that better crystalline samples were formed with an excess of the amine.

Layered bilayers of tungsten oxide hydrate, $\mathrm{H}_{2} \mathrm{~W}_{2} \mathrm{O}_{7}$, can be formed by soaking the Aurivillius phase material $\mathrm{Bi}_{2} \mathrm{~W}_{2} \mathrm{O}_{9}$ in $\mathrm{HCl}$ for several days to remove the $\mathrm{BiO}_{2}$ zig-zag sheets [152]. Dissolving this in solution and recrys- 
tallising would form the monolayer structure, so this material was used in a melt method experiment in an attempt to form the equivalent bilayer hybrid. This experiment was unsuccessful, also forming the monolayer hybrid. We have yet to attempt chimie douce reactions on this material.

The wide variety of possible organic intercalates is one of the attractive features of the organic-inorganic hybrid system. So far we have only investigated simple aliphatic mono- and di-amines and a few simple aromatic amines. Functional organic molecules such as dyes $[46,196]$ have been successfully intercalated into metal halide hybrids, so one would expect similar molecules could also be intercalated in the tungsten oxide system. Various functionalities could be included via the incorporation of luminescent molecules, conducting molecules or polymers, metal complexes (e.g. porphyrins), and so forth.

There is also the possibility of altering the terminal group from the amine terminations used throughout this work to groups such as sulphates, phosphates, and carboxylates. The strength of the interaction between the organic terminal group and the inorganic layer is observed to affect the structure and the physical properties (one example being the changes in the optical spectra of the transition metal tungstate hybrids as the metal-amine interaction strengthens).

Other synthetic techniques could also be used to attempt to produce the hybrids, for example, hydrothermal techniques at high temperatures and/or pressures. Extreme synthesis conditions like this may be what is required to 'force' bulkier molecules (e.g. DAphen) into the tungsten oxide layer structure, which are not able to be formed with the standard techniques used so far. Layer-by-layer assembly could also provide an excellent means for fabricating thin epitaxial films on lattice-matched substrates.

A major obstacle of the project to date is the absence of any definite crystal structure. The best method for determining crystal structures is via single crystal X-ray diffraction, which requires crystals of $\geq 200 \mu \mathrm{m}$ to a side. Smaller crystals (down to $50 \mu \mathrm{m}$ to a side) can also be examined using synchrotron techniques, although the X-rays involved are more energetic and potentially damaging to samples with organic components. 
The lack of suitably large single crystals prompted the experiments in electron diffraction to combine the results with those from powder XRD. The presence of a supercell suggests that the initial indexing of the unit cell is probably more complex than anticipated. Neutron diffraction is another avenue of exploration, and while this requires deuterated organic species (as ${ }^{1} \mathrm{H}$ is a negative scatterer, resulting in spectra with very low signal-to-noise ratios), work is underway to produce such samples for analysis.

One of the questions that arose primarily from the IR and Raman analysis (but was also alluded to in the microanalysis and TGA) was the disposition of the protons in W-DA2 compared with the longer chain W-DAn hybrids. It is assumed that two extra protons per $\mathrm{WO}_{4}{ }^{2-}$ unit would be required for charge balance. In the longer chain DAn molecules the amine terminations are protonated but in the shortest DA2 case they are not. One suggestion is that because of the short interlayer distance in W-DA2, the protons dissociate from the terminal amine groups and relocate to the space between facing apical oxygens, thus 'shielding' the electrostatic repulsion. At the time of writing, samples have been sent to ANSTO for solid state ${ }^{1} \mathrm{H}$ NMR experiments. It is hoped that the results will enable us to determine the environment of each proton. If the protons are delocalised then the system could represent a 2-dimensional ionic proton conductor, with doping.

Ab initio calculations could also be performed for protons in various locations and configurations to explore the possibility of proton mobility in W-DA2. Work along this vein was begun but not completed in the time available. Calculations of energy barriers in hopping from one site to another are quite time-intensive, and combined with the uncertainty of the hydrogen wavefunction used (as VASP handles hydrogen poorly) it was decided not to pursue these calculations at the time.

Calculations were also attempted on W-DAphen, without success due to apparent charge imbalance from the saturated bonds of the benzene ring. These problems could be resolved by using another ab initio program. 


\subsection{Transition metal tungstates}

The synthesis and characterisation of the transition metal tungstates began quite late in the project, with the first manganese samples synthesised in August 2003 and the other transition metal ions studied beginning in May 2004. Each of the systems have different chemistries and optimisation is required for each one. The limitation on some of the hybrids (in particular, cobalt, nickel and copper) with regards to synthetic techniques is a major factor to be explored. There are many parameters that can be altered to affect the quality of the final outcome using the chimie douce and 'melt' methods, such as stoichiometry, $\mathrm{pH}$, temperature, solvents, and washing and drying procedures. Other synthetic techniques, in particular hydrothermal techniques, could be attempted to try to force the formation of metastable phases (e.g. spin ladder compounds).

The transition metal tungstate inorganic layer can also be combined with the functional organic molecules suggested for the 'pure' tungsten oxide hybrids - chromophores, luminescent molecules, conducting molecules and polymers, metal complexes, and so on. (The apparent 'stripe' spacing in the TEM results is comparable with that of polyaniline $(\sim 10 \AA)$ which would create an interesting system with the possibility of two-band conduction, self-doping and stronger electronic coupling between the layers. A similar structure was obtained for a vanadium oxide hybrid [98].)

$A b$ initio calculations on the proposed transition metal tungstate structures could also be performed, and to measure the difference in the metalamino group interaction strength as the metal ion is altered.

Once again, determination of the crystal structure is of utmost importance and so far unsuccessful due to the small size of the crystals produced. Fully deuterated samples will be prepared in the near future for powder neutron diffraction studies. Some optimisation of crystal size was attempted by varying the $\mathrm{pH}$ of synthesis (the optimal $\mathrm{pH}$ was found to be 9-10).

Of the transition metal hybrids, (Mn,W)-DA6 is observed to form the largest crystals (under certain synthesis conditions) of platelets up to $10 \mu \mathrm{m}$ across. These may be large enough to attempt single crystal XRD using a 
synchrotron source. If successful, the same inorganic structure is known to be present in the other $(\mathrm{Mn}, \mathrm{W})-\mathrm{DAn}$ hybrids and $\mathrm{MnWO}_{4} \cdot n \mathrm{H}_{2} \mathrm{O}$.

For the other samples, the (M,W)-DAn series are still incomplete. This has been limited mostly by the inability to form longer chain hybrids using the melt and chimie douce methods. The (M,W)-DAn series was used in the manganese tungstate and tungsten oxide hybrid cases to determine structural information about the packing angle of the organic molecules between the layers and the thickness of the inorganic layers.

High-resolution TEM images on the (Mn,W)-DAn samples provided a key to proposing a structure. High-resolution TEM only became available late in this project with the commissioning of the MacDiarmid Institute instrument. This tool should also be applied to the other transition metal tungstate hybrids if possible, along with electron diffraction which gives information specific to the $a b$ plane, for instance, not immediately discernable from the powder XRD spectra. All of these techniques can then be used simultaneously to build up a reasonable structural model, as demonstrated in Chapter 5 for the manganese hybrid.

Other experiments were begun but not completed for the transition metal hybrids, such as IR and Raman spectroscopy and TGA, which give indications of the bonding strength within the samples and their structural and thermal stability.

It would also be worthwhile to perform conductivity experiments on the transition metal tungstate hybrids and/or thermopower. An initial attempt was made to measure the conductance of pressed pellets of the manganese tungstate hybrids, however the conductance was too low to measure with the equipment available to us. This would be an important physical property to measure especially when doping is achieved in the samples.

\subsection{Magnetism}

The magnetic measurements on the transition metal tungstate hybrids demonstrate how rich the physical behaviour of supposedly related systems can be. The measurements presented in Chapter 6 provide a starting point for 
future experiments - not excluding future magnetic characterisation of materials with more exotic functional organic intercalates.

The proposed structure of the manganese tungstate hybrids indicates it may form a spin ladder compound (although the high-temperature part of the DC susceptibility can be fitted very well by a Heisenberg AF $S=\frac{5}{2}$ chain model). Spin ladder cuprates are the focus of much recent attention because these compounds, when properly doped, exhibit superconductivity. They also form a conceptual template for understanding HTS cuprates which appear to spontaneously charge-segregate into stripe-like structures. Such a model reproduces the full $\epsilon(\mathbf{q})$ dispersion for the magnetic excitations in HTS cuprates probed by inelastic neutron scattering. The present spin-ladder hybrids would exhibit 2-magnon scattering peaks in low-temperature Raman experiments. This would also be a unique compound among spin ladder materials, which mostly have $S=\frac{1}{2}\left(\mathrm{Cu}^{2+}, \mathrm{V}^{3+}\right)$. Interesting magnetic behaviour may also be observed if the other transition metals could also be forced (e.g. through hydrothermal synthesis) to adopt this spin ladder structure.

Magnetic alignment of the grains is another area of research which would provide additional information, through measuring the magnetisation parallel to and perpendicular to the layers, essentially independently. One would expect to see differences due to the different exchange interactions $J_{\|}$and $J_{\perp}$ respectively. Standard commercial resin uses an amine-based curing agent which disrupts the hybrid structure. Therefore a medium such as a wax or silicone polymer with a relatively low melting point $\left(30-40^{\circ} \mathrm{C}\right)$ would be a better material to use. In this way one might observe a spin-flop transition when the field is applied in the plane of orientation of the spins.

NMR is another technique that could be applied to all of the transition metal tungstate hybrids to obtain additional information about any net magnetic order. Below the transition temperature one would expect to see a zero-field NMR signal, indicative of net magnetic order. If the feature is broad, this corresponds to different sites with different hyperfine fields. If it is a sharp peak, then the material is of a single phase and is well-ordered. 


\subsection{Doping}

Although Chapter 7 on doping is the shortest chapter here, this actually represents the largest obstacle to overcome in order to use the organic-inorganic hybrid materials in electronic applications. As mentioned in Chapter 7, electrochemistry is only one of several possible means of doping the samples. This technique is not yet fully optimised, and preliminary results on the manganese tungstate hybrids indicate that this may well be successful in these samples.

Measuring the change in conductance of doped samples is an obvious first step to characterise their new properties. This can be done either in situ or afterwards. IR reflectance measured over a wide frequency range would be a priority, as at low frequencies one ought to see the development of a Drude peak corresponding to increased conduction. Measurements of photoinduced conductivity would help further characterise the electronic structure and provides another way of injecting mobile carriers.

Preliminary work has begun on the fabrication and testing of thin-film field-effect transistor devices to simultaneously dope and measure conduction of the hybrid materials. This has proven to be more difficult than anticipated, however, field-effect behaviour has been observed in a thin film device based on a tungsten oxide hydrate material.

\subsection{Soft-mode behaviour}

The fact that the organic molecule has several available modes of packing adjacent to the structural units of the inorganic layer, both in the positioning of the amine groups and the orientation of the molecule, indicates the possibility of local activation and the occurrence of order/disorder transitions. If the energy barriers between different configurations are as small, as we expect, then one should observe a low-temperature ordered state with a transition to the disordered state at elevated temperature. In such a scenario one may observe soft-mode behaviour as a vibrational mode associated with the lowtemperature symmetry slows and softens. Such behaviour could be observed 
using an ultrasonic probe and the reduction in symmetry observed in x-ray diffraction as well as by the demise of specific phonon modes observed by IR or Raman spectroscopy. The magnitude of these barriers could be estimated using ab initio computations.

\subsection{Conclusion}

This thesis presents a thorough study of the synthesis and characterisation of the chemical, structural, and physical properties of a novel class of hybrid material based on atomic layers of a metal oxide separated by organic amine spacer molecules. The hybrid materials can be produced via evaporation of solvent, chimie douce, and the so-called 'melt' methods. For the synthesis of tungsten oxide and manganese tungstate hybrids the evaporation of solvent method was favoured, although for cobalt, nickel and copper tungstate hybrids the other two methods are necessary due to the formation of ammonia complexes with the transition metal ions.

For the majority of the compounds studied (the cobalt, nickel and copper tungstate hybrids with 1,2-diaminoethane as the only exceptions), a series of intense $00 \ell$ lines was evident in powder X-ray diffraction patterns, indicative of the presence of layered structures. For the tungsten oxide-diaminoalkane hybrid series, the structure was determined to comprise of single atomic layers of corner-shared $\mathrm{WO}_{6}$ octahedra, with the organic molecules aligning perpendicular to the inorganic layers. For the manganese tungstate-diaminoalkane hybrid series, the organic molecules were found to lie at an angle of $56.5^{\circ}$ to the inorganic layer, while the inorganic layer itself is thought to be comprised of alternating 'stripes' of manganese and tungsten oxide in a bilayer formation. IR and Raman spectroscopy of these two systems indicate that within each series the compounds are isostructural, which assists greatly in comparing their physical properties.

$A b$ initio calculations were performed on the tungsten oxide and tungsten bronze series, which agreed extremely well with both experimental results and computational results obtained by other researchers. This was extended to the tungsten oxide hybrids and the optimal structure obtained. The density 
of states for the hybrids near the Fermi level was found to be independent of the interlayer spacing, and was related to the direct and indirect optical transitions observed in powder and thin-film UV-visible spectroscopy. Optical spectroscopy performed on tungsten oxide hybrids incorporating transition metal ions appeared to indicate strong interactions between the transition metal ions and the amine terminations of the organic molecules.

The transition metal tungstate hybrids also exhibit magnetism due to the presence of unpaired electrons. The manganese tungstate hybrid series with diaminoalkanes exhibited an antiferromagnetic transition at low temperatures $(T<12 \mathrm{~K})$ that decreased as the interlayer spacing increased. This corresponds to the reduced coupling expected as the layers are separated. The iron tungstate hybrid series shows all the features of a spin glass, in particular strong frequency-dependence of the AC susceptibility. A copper tungstate hybrid with 1,6-diaminohexane as the organic intercalate displayed a DC susceptibility curve that was described well by a spin-ladder model with very weak coupling along the ladder. A nickel tungstate hybrid, also with 1,6-diaminohexane as the organic spacer, displayed a ferromagnetic transition which was subsequently attributed to a spin-canted antiferromagnetic phase with a small canting angle of $0.46^{\circ}$.

These materials have been shown to offer a wide range of interesting structural, optical, electronic and magnetic behaviours that arise from the presence of essentially isolated inorganic layers. They represent a model system that is easily manipulated by the selection of the organic molecule to exploit the low-dimensional physics associated with the layered structures. The present work provides a 'first look' at this rich and fascinating system, and there is much that can yet be done to develop a full understanding of their properties, and to exploit these properties to expose new physics and applications. 


\section{Appendix A}

\section{Literature Examples of Hybrid Systems}

\begin{tabular}{|l|l|}
\hline Abbreviation & Name \\
\hline evap & evaporation \\
evap solv & evaporation of solvent \\
ppt & precipitate \\
cryst sol & crystallisation from solution \\
el-chem cryst grow & electrochemical crystal growth \\
SSTA & single-source thermal evaporation \\
DSTA & double-source thermal evaporation \\
\hline
\end{tabular}

Table A.1: Abbreviations of techniques used in this section 


\begin{tabular}{|c|c|c|}
\hline Abbreviation & Name & Chemical formula \\
\hline MAn $(\mathrm{n}=1,2, \ldots)$ & methyl/ethyl/...-amine & $\mathrm{H}_{2} \mathrm{~N}\left(\mathrm{CH}_{2}\right)_{n-1} \mathrm{CH}_{3}$ \\
\hline DAn $(\mathrm{n}=2,3,4, \ldots)$ & diamino-eth/prop/... -ane & $\mathrm{H}_{2} \mathrm{~N}\left(\mathrm{CH}_{2}\right)_{n} \mathrm{NH}_{2}$ \\
\hline bipy & bipyridine & $\left(\mathrm{NC}_{5} \mathrm{H}_{4}\right)_{2}$ \\
\hline Py & pyridine & $\mathrm{NC}_{5} \mathrm{H}_{5}$ \\
\hline $\mathrm{AEQT}$ & bis(aminoethyl)quaterthiophene & $\mathrm{H}_{2} \mathrm{~N}\left(\mathrm{CH}_{2}\right)_{2}\left(\mathrm{C}_{4} \mathrm{SH}_{2}\right)_{4} \mathrm{CH}_{2} \mathrm{NH}_{2}$ \\
\hline AETH & aminoethylthienylhexane & $\mathrm{H}_{2} \mathrm{~N}\left(\mathrm{CH}_{2}\right)_{2}\left(\mathrm{C}_{4} \mathrm{SH}_{2}\right)\left(\mathrm{CH}_{2}\right)_{6^{-}}$ \\
\hline EDT-TTF & ethylenedithiotetrathiafulvalene & \\
\hline tmeda & tetramethylethylenediamine & $\left(\mathrm{CH}_{3}\right)_{2} \mathrm{~N}\left(\mathrm{CH}_{2}\right)_{2} \mathrm{~N}\left(\mathrm{CH}_{3}\right)_{2}$ \\
\hline phen & phenethylamine & $\left(\mathrm{C}_{6} \mathrm{H}_{5}\right)\left(\mathrm{CH}_{2}\right)_{2} \mathrm{NH}_{2}$ \\
\hline 5FPEA & pentafluorophenethylammonium & $\left(\mathrm{C}_{6} \mathrm{~F}_{5}\right)\left(\mathrm{CH}_{2}\right)_{2} \mathrm{NH}_{3}$ \\
\hline NEA & 2-napththyleneethylammonium & $\left(\mathrm{C}_{10} \mathrm{H}_{7}\right)\left(\mathrm{CH}_{2}\right)_{2} \mathrm{NH}_{3}$ \\
\hline BAESBT & bis(ammoniumethylsulfanyl)- & $\mathrm{H}_{3} \mathrm{~N}\left(\mathrm{CH}_{2}\right)_{2} \mathrm{~S}_{(}\left(\mathrm{C}_{4} \mathrm{SH}_{2}\right)_{2^{-}}$ \\
\hline pip & piperazine & $\left(\mathrm{C}_{4} \mathrm{~N}_{2}\right) \mathrm{H}_{4}$ \\
\hline
\end{tabular}

Table A.2: Abbreviations of organic molecules used in this section. 


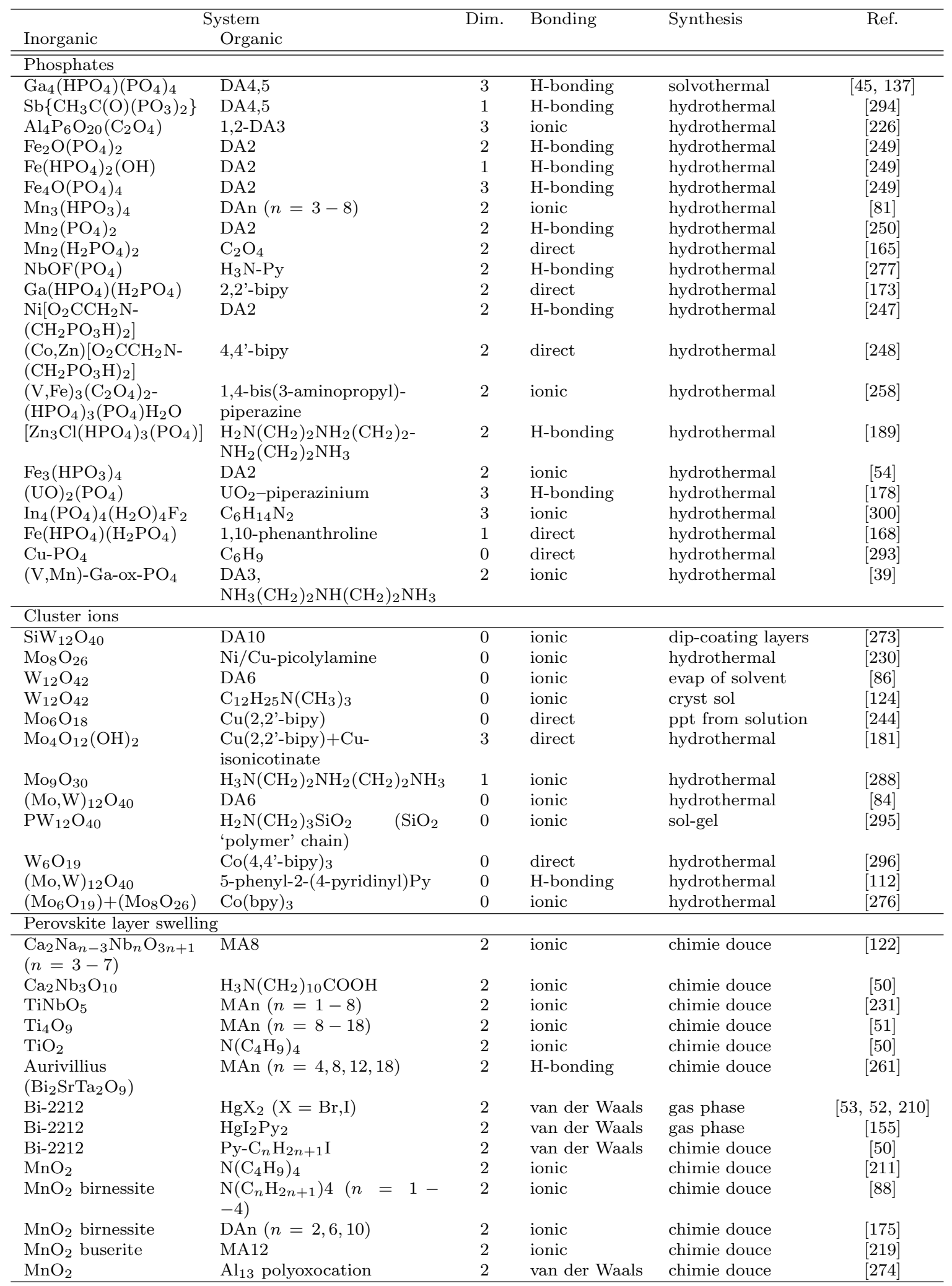




\begin{tabular}{|c|c|c|c|c|}
\hline Inorganic & $\begin{array}{l}\text { System } \\
\text { Organic }\end{array}$ & Bonding & Synthesis & Ref. \\
\hline \multicolumn{2}{|l|}{ LDHs/anionic clays: } & \multicolumn{3}{|l|}{$\overline{\text { Dim. }=2}$} \\
\hline Al-Si-O clay & poly-diallylammonium species & ionic & dip-coat layer & {$[270]$} \\
\hline Al-Si-O clay & biomolecules (DNA, vit. C, ...) & ionic & chimie douce & {$[50]$} \\
\hline $\begin{array}{l}\mathrm{Ni}_{0.7} \mathrm{M}_{0.3} \\
\mathrm{Co}, \mathrm{Mn})\end{array} \quad(\mathrm{M}=\mathrm{Fe}$, & polyacrylate & ionic & ppt from solution & [268] \\
\hline $\begin{array}{l}\mathrm{M}_{2} \mathrm{Al}(\mathrm{OH})_{6}(\mathrm{M}=\mathrm{Zn}, \\
\mathrm{Mg})\end{array}$ & M-oxalate $(\mathrm{M}=\mathrm{Co}, \mathrm{Cu}, \mathrm{Mn}, \mathrm{Ga})$ & ionic & hydrothermal & {$[22]$} \\
\hline $\mathrm{Zn}_{2.9} \mathrm{Al}_{2}(\mathrm{OH})_{9.8}$ & $\begin{array}{l}\operatorname{bipy}\left(\mathrm{COO}_{2}\right) \\
\operatorname{bipy}\left(\mathrm{COO}_{2}\right)+\mathrm{MoO}_{2}\end{array}$ & van der Waals & ppt from solution & {$[85]$} \\
\hline \multirow{2}{*}{$\begin{array}{l}\mathrm{M}_{2} \mathrm{Al}(\mathrm{OH})_{6} \quad(\mathrm{M}=\mathrm{Zn} \\
\mathrm{Mg}, \mathrm{Cu}) \\
\mathrm{Mg}-\mathrm{Al}-\mathrm{NO}_{3}\end{array}$} & {$\left[\mathrm{M}^{\prime} \mathrm{Cl}_{6}\right]^{3-}\left(\mathrm{M}^{\prime}=\mathrm{Ir}, \mathrm{Pt}, \mathrm{Ru}\right)$} & ionic & hydrothermal & {$[23]$} \\
\hline & $\mathrm{Eu}(\mathrm{EDTA})$ & ionic & ppt from solution & [168] \\
\hline \multicolumn{5}{|l|}{ Other $1 \mathrm{D}$ chains } \\
\hline $\mathrm{Cd}_{2}(\mathrm{SCN})_{6}$ & $\begin{array}{l}\text { (Me/cyclohexane })_{2-} \\
\text { imidazolium }\end{array}$ & ionic & cryst sol & {$[42]$} \\
\hline \multirow{3}{*}{$\begin{array}{l}\mathrm{CuCl}_{2} \\
\mathrm{CuX}(\mathrm{X}=\mathrm{Cl}, \mathrm{Br}, \mathrm{I}) \\
\mathrm{Pb}_{3} \mathrm{X}_{9}(\mathrm{X}=\mathrm{Cl}, \mathrm{Br}) \\
\text { (edge shared) }\end{array}$} & o-phenanthroline & \multirow{3}{*}{$\begin{array}{l}\text { direct } \\
\text { direct } \\
\text { ionic }\end{array}$} & \multirow{3}{*}{$\begin{array}{l}\text { hydrothermal } \\
\text { cryst sol } \\
\text { cryst sol }\end{array}$} & \multirow{2}{*}[256]{} \\
\hline & 4-acetylpyridine & & & \\
\hline & $\left(\mathrm{C}_{2} \mathrm{H}_{5}\right)_{2} \mathrm{NH}_{2}$ & & & $\begin{array}{l}{[37]} \\
{[282]}\end{array}$ \\
\hline $\mathrm{V}_{2} \mathrm{SeO}_{4}$ & 2,2'-bipy, 4,4'-bipy & direct & hydrothermal & {$[65]$} \\
\hline $\mathrm{Mo}_{4} \mathrm{O}_{13}$ & $\mathrm{Cu}(2,2$ '-bipy $)+$ terephthalate & direct & hydrothermal & [184] \\
\hline $\mathrm{Mo}_{3} \mathrm{O}_{10}$ & anilinium & ionic & $?$ & {$[157]$} \\
\hline $\mathrm{WO}_{2} \mathrm{~F}_{4}$ & $\mathrm{Cu}(\mathrm{Py})_{2}\left(\mathrm{H}_{2} \mathrm{O}\right)_{2}$ & direct & solvothermal & [110] \\
\hline $\mathrm{MoO}_{4}$ & Mn-o-phenanthroline & direct & hydrothermal & {$[297]$} \\
\hline$\left(\mathrm{MoO}_{3}\right)_{3} \mathrm{SO}_{4}$ & 2-methylpiperazine $\left(\mathrm{C}_{5} \mathrm{~N}_{2} \mathrm{H}_{1} 4\right)$ & H-bonding & hydrothermal & {$[107]$} \\
\hline $\mathrm{MO}_{5}(\mathrm{M}=\mathrm{Ce}, \mathrm{Sm})$ & $\begin{array}{l}\mathrm{HOOC}\left(\mathrm{CH}_{2}\right)_{2} \mathrm{COOH} \text { (succinic } \\
\text { acid) }\end{array}$ & direct & hydrothermal & {$[242]$} \\
\hline $\mathrm{Cu}_{2}\left(\mathrm{IO}_{3}\right)_{3}$ & 2,2'-bipy & direct & hydrothermal & [169] \\
\hline $\mathrm{Zn}_{3}(\mathrm{OH})_{2}$ & $\mathrm{OOC}\left(\mathrm{C}_{6} \mathrm{H}_{4}\right) \mathrm{COO}$ & direct & hydrothermal & [179] \\
\hline Other 2D layers & & & & \\
\hline $\mathrm{V}_{6} \mathrm{O}_{14}$ & DA4 & H-bonding & hydrothermal & [239] \\
\hline$(\mathrm{Pb}, \mathrm{Ag}) \mathrm{I}_{3}$ & EDT-TTF-I 2 & van der Waals & el-chem crys grow & {$[73]$} \\
\hline (tmeda) $\mathrm{Cu}\left[\mathrm{Hg}(\mathrm{CN})_{2}\right]_{2}[\mathrm{Hg}$ & $\left.\mathrm{gCl}_{4}\right]$ & direct & ppt from solution & {$[75]$} \\
\hline $\begin{array}{l}(\mathrm{ZnO})_{2-}^{-} \\
\left(\mathrm{UO}_{2}\right)_{3}(\text { acetic })_{2}\end{array}$ & nicotinic acid & direct & hydrothermal & {$[43]$} \\
\hline $\mathrm{Co}-\mathrm{W}(\mathrm{CN})_{8}$ & cyanopyridine & direct & ppt from solution & {$[7]$} \\
\hline $\mathrm{Cr}\left[\left(\mathrm{H}_{3} \mathrm{~N}\left(\mathrm{CH}_{2}\right)_{2} \mathrm{PO}_{3}\right)(\mathrm{Cl})\right.$ & $\left.\left(\mathrm{H}_{2} \mathrm{O}\right)\right]$ & direct & ppt from solution & {$[21]$} \\
\hline $\mathrm{SnI}_{3}$ (c.f. $\mathrm{MoO}_{3}$ ) & MA12 & H-bonding & evap of solvent & [289] \\
\hline $\mathrm{Co}_{5}(\mathrm{OH})_{8}$ & cyclohexanedicarboxylate & direct & hydrothermal & {$[153]$} \\
\hline $\mathrm{V}_{4} \mathrm{O}_{12}$ & $\mathrm{M}\left(2,2^{\prime}\right.$-bipy $)(\mathrm{M}=\mathrm{Cu}, \mathrm{Zn})$ & direct & hydrothermal & [109] \\
\hline $\mathrm{MoO}_{4}$ & $\mathrm{Cu}\left(\mathrm{n}, \mathrm{m}^{\prime}\right.$-bipy $)(n, m=3,4)$ & direct & hydrothermal & [229] \\
\hline $\mathrm{Ag}_{2} \mathrm{SbS}_{3}, \mathrm{Ag}_{5} \mathrm{Sb}_{3} \mathrm{~S}_{8}$ & $\mathrm{DA} 2$ & H-bonding & solvothermal & {$[263]$} \\
\hline $\mathrm{V}_{3} \mathrm{O}_{7}, \mathrm{~V}_{4} \mathrm{O}_{1} 0$ & MA1 & ionic & hydrothermal & {$[41]$} \\
\hline $\mathrm{V}_{10} \mathrm{O}_{26}$ & $\mathrm{Co}(\text { phenanthroline })_{3}$ & ionic & hydrothermal & {$[182]$} \\
\hline $\mathrm{Fe}_{1.5} \mathrm{~F}_{3} \mathrm{SO}_{4}$ & DA6 & H-bonding & solvothermal & {$[228]$} \\
\hline $\mathrm{V}_{x} \mathrm{O}_{y}$ & conducting polymers & ionic & hydrothermal & {$[98]$} \\
\hline$\left(\mathrm{UO}_{2}\right)\left(\mathrm{AsO}_{4}\right)$ & triethylenediammonium & H-bonding & hydrothermal & [178] \\
\hline$\left(\mathrm{UO}_{2}\right)_{2}\left(\mathrm{AsO}_{4}\right)\left(\mathrm{AsO}_{3} \mathrm{OH}\right)$ & $\mathrm{NH}\left(\mathrm{C}_{2} \mathrm{H}_{5}\right)_{3}$ & H-bonding & hydrothermal & {$[178]$} \\
\hline $\mathrm{V}_{2} \mathrm{O}_{6}, \mathrm{~V}_{4} \mathrm{O}_{12}$ & $\mathrm{Co}(4,4$ '-bipy $)$ & direct & hydrothermal & [135] \\
\hline $\mathrm{U}_{2} \mathrm{~F}_{10}$ & $\mathrm{~N}\left(\mathrm{CH}_{2} \mathrm{CH}_{2} \mathrm{NH}_{3}\right)_{3}$ & ionic & hydrothermal & {$[272]$} \\
\hline Other 3D r & & & & \\
\hline $\mathrm{ZrGe}_{3} \mathrm{O}_{9}$ & DA2 & ionic & hydro/solvotherm & [176] \\
\hline
\end{tabular}

Table A.3: Examples of organic-inorganic hybrids of various dimensionality. 


\begin{tabular}{|c|c|c|c|c|c|}
\hline Inorganic & $\begin{array}{l}\text { System } \\
\text { Organic }\end{array}$ & Dim. & Bonding & Synthesis & Ref. \\
\hline$\overline{\mathrm{Sn}_{n} \mathrm{I}_{3 n+1}, n=1-5}$ & DA4 & 2 & H-bonding & cryst sol & [200] \\
\hline $\mathrm{SnI}_{3}$ & $\mathrm{NH}_{2} \mathrm{CH}=\mathrm{NH}_{2}$ & 3 & H-bonding & cryst sol & [203] \\
\hline $\mathrm{MI}_{5}(\mathrm{M}=\mathrm{Sn}, \mathrm{Pb})$ & $\mathrm{NH}_{2} \mathrm{C}(\mathrm{I})=\mathrm{NH}_{2}$ & 1 & H-bonding & cryst sol & {$[275]$} \\
\hline $\mathrm{SnI}_{3}$ & MA1 & 3 & H-bonding & cryst sol & [201] \\
\hline $\mathrm{MI}_{4}(\mathrm{M}=\mathrm{Ge}, \mathrm{Sn}, \mathrm{Pb})$ & MA4 & 2 & H-bonding & cryst sol & {$[195]$} \\
\hline $\mathrm{M}_{\frac{2}{3}} \mathrm{I}_{4}(\mathrm{M}=\mathrm{Bi}, \mathrm{Sb})$ & AEQT dye & 2 & H-bonding & cryst sol & {$[197,46]$} \\
\hline $\mathrm{PbI}_{4}^{3}$ & phen, MAn $(n=4,6,10)$ & 2 & H-bonding & DSTA & {$[78]$} \\
\hline $\mathrm{MX}_{4}$ various & phen, MA4 & 2 & H-bonding & SSTA & [205] \\
\hline $\mathrm{PbX}_{4}(\mathrm{X}=\mathrm{Br}, \mathrm{I})$ & AETH & 2 & H-bonding & SSTA & {$[47]$} \\
\hline $\begin{array}{l}\mathrm{M}_{n} \mathrm{I}_{3 n+1}(\mathrm{M}=\mathrm{Pb}, \mathrm{Sn} \\
n=1,2, \infty)\end{array}$ & DA4, phen & 2 & H-bonding & 2 step dip & {$[171]$} \\
\hline $\mathrm{PbX}_{3}(\mathrm{X}=\mathrm{Cl}, \mathrm{Br}, \mathrm{I})$ & MA1 & 3 & H-bonding & cryst sol & {$[186,279]$} \\
\hline $\mathrm{EuI}_{4}$ & MA4 & 2 & H-bonding & solid state & {$[202]$} \\
\hline $\mathrm{CuX}_{4}(\mathrm{X}=\mathrm{Cl}, \mathrm{Br})$ & $\mathrm{H}_{3} \mathrm{~N}$-pyridinium/morpholinium & 2 & H-bonding & cryst sol & {$[283]$} \\
\hline $\mathrm{CuX}_{4}(\mathrm{X}=\mathrm{Cl}, \mathrm{Br})$ & $\mathrm{N}_{3} \mathrm{~N}$-picolinium & 2 & H-bonding & cryst sol & [180] \\
\hline $\mathrm{MnCl}_{4}$ & MA2, MA9 & 2 & H-bonding & solid state & [287] \\
\hline $\mathrm{SnI}_{4}\langle 330\rangle$ & DA5 & 2 & H-bonding & cryst sol & [103] \\
\hline $\mathrm{CuX}_{4}(\mathrm{X}=\mathrm{Cl}, \mathrm{Br})$ & DA 4,5 & 2 & H-bonding & cryst sol & {$[90]$} \\
\hline $\begin{array}{l}\mathrm{MCl}_{4}(\mathrm{M}=\mathrm{Ni}, \mathrm{Pd}, \mathrm{Cu} \\
\mathrm{Cd}, \mathrm{Mn})\end{array}$ & DA2 & 2 & H-bonding & cryst sol & {$[246]$} \\
\hline $\mathrm{SnI}_{4}$ & 5FPEA, NEA & 2 & H-bonding & cryst sol & {$[290,204]$} \\
\hline $\mathrm{SnI}_{4}$ & (CH3)3N(CH2)2NH3 & 2 & H-bonding & evap solv & [292] \\
\hline $\mathrm{SnI}_{4}$ & 2-Xphen $(\mathrm{X}=\mathrm{F}, \mathrm{Cl}, \mathrm{Br})$ & 2 & H-bonding & cryst sol & [291] \\
\hline $\mathrm{PbI}_{4}$ & BAESBT & 2 & H-bonding & hydrothermal & [299] \\
\hline $\mathrm{PbX}_{4}(\mathrm{X}=\mathrm{Cl}, \mathrm{Br})$ & $\begin{array}{l}\text { piperazinium } \\
(\mathrm{Cl}, \mathrm{Br})\end{array}$ & 2 & H-bonding & cryst sol & {$[59]$} \\
\hline $\mathrm{BiI}_{5}$ & AETH, DA12, DA6 & 1 & H-bonding & cryst sol & [198] \\
\hline $\mathrm{PbCl}_{4}$ & $\begin{array}{l}\mathrm{H}_{2} \mathrm{~N}\left(\mathrm{CH}_{2}\right)_{3} \mathrm{SiO}_{2} \quad\left(\mathrm{SiO}_{2} \text { 'poly- }\right. \\
\text { mer' chain) }\end{array}$ & 2 & H-bonding & ppt from sol & {$[44]$} \\
\hline $\mathrm{PbI}_{5}$ & BAESBT & 1 & H-bonding & cryst sol & [298] \\
\hline $\mathrm{CuCl}_{4}$ & MA1 & 2 & H-bonding & cryst sol & [216] \\
\hline $\mathrm{PbX}_{4}(\mathrm{X}=\mathrm{Br}, \mathrm{I})$ & $\mathrm{HO}\left(\mathrm{CH}_{2}\right)_{2} \mathrm{NH}_{3}$ & 2 & H-bonding & ppt from sol & [193] \\
\hline
\end{tabular}

Table A.4: Examples of metal halide organic-inorganic hybrids. 
188APPENDIX A. LITERATURE EXAMPLES OF HYBRID SYSTEMS 


\section{Appendix B}

\section{VASP Input Files}

These are the actual input files for an ab initio relaxation, using VASP, of the W-DA4 system using a single unit cell.

\section{POSCAR}

The atomic positions are given as follows, in the format:

- System: - a comment line;

- Unit cell multiplication factor;

- Unit cell basis vectors (each as $(x, y, z))$;

- The number of atoms of each species as listed in the POTCAR file;

- Selective dynamics - optional. This allows one to vary or fix the position of certain atoms, if desired;

- Direct - direct, as opposed to reciprocal, co-ordinate system;

- Atomic positions, as fractions of the unit cell basis vectors. If Selective dynamics is selected, each co-ordinate must be specified as to whether it is allowed to vary (' $T$ ') or remain fixed (' $F$ '). 


\begin{tabular}{|c|c|c|c|c|c|c|c|}
\hline \multicolumn{8}{|c|}{$\begin{array}{l}\text { System: W04-DA4 single cell, terminal } \\
\quad 3.937256000000000\end{array}$} \\
\hline \multicolumn{2}{|c|}{1.0017983395056560} & \multicolumn{2}{|c|}{-0.0003952000493081} & \multicolumn{3}{|c|}{0.0000834421981564} & \\
\hline \multicolumn{2}{|c|}{-0.0011295670591459} & \multicolumn{2}{|c|}{2.8639602762709550} & \multicolumn{3}{|c|}{0.0012995157463463} & \\
\hline \multicolumn{2}{|l|}{0.0000833769576506} & \multicolumn{2}{|c|}{0.0004582493508579} & \multicolumn{3}{|c|}{1.0009946034456180} & \\
\hline $\begin{array}{lll}2 & 14 & 1\end{array}$ & & & & & & & \\
\hline \multicolumn{8}{|l|}{ Selective dynamics } \\
\hline \multicolumn{8}{|l|}{ Direct } \\
\hline 0.6243605412157436 & & 323433567902825 & 0.45 & 53656839381 & $\mathrm{~T}$ & $\mathrm{~T}$ & $\mathrm{~T}$ \\
\hline 0.3764915036686958 & & 638525836721527 & 0.500 & 3575134924105 & $\mathrm{~T}$ & $\mathrm{~T}$ & $\mathrm{~T}$ \\
\hline 0.6021454597081402 & & 552109454410044 & 0.498 & 3663555382591 & $\mathrm{~T}$ & $\mathrm{~T}$ & $\mathrm{~T}$ \\
\hline 0.3972100340530247 & & 405334336655287 & 0.495 & 3042004666772 & $\mathrm{~T}$ & $\mathrm{~T}$ & 1 \\
\hline 0.4414292644498232 & 0.2 & 185203690990178 & 0.496 & 5748819565263 & $\mathrm{~T}$ & $\mathrm{~T}$ & $\mathrm{~T}$ \\
\hline 0.5594312623738347 & 0.7 & 778810794278641 & 0.495 & 3641643899593 & 1 & $\mathrm{~T}$ & $\mathrm{~T}$ \\
\hline 0.2820933524270117 & 0.2 & 052294135958732 & 0.280 & 0348900693947 & $\mathrm{~T}$ & $\mathrm{~T}$ & $\mathrm{~T}$ \\
\hline 0.2797722622929228 & 0.2 & 051479976316344 & 0.712 & 3877832720140 & 1 & $\mathrm{~T}$ & $\mathrm{~T}$ \\
\hline 0.6145554510235272 & & 501346846160700 & 0.497 & 8301846778663 & $\mathrm{~T}$ & $\mathrm{~T}$ & $\mathrm{~T}$ \\
\hline 0.7893609199975202 & 0.3 & 307991520295701 & 0.720 & 0110169246656 & 1 & $\mathrm{~T}$ & $\mathrm{~T}$ \\
\hline 0.7869051395585771 & & 317253904940998 & 0.272 & 043638308098 & 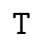 & $\mathrm{T}$ & $\mathrm{T}$ \\
\hline 0.7217668433961961 & 0.7 & 918226361883853 & $0.71<$ & 516189173681 & 1 & $\mathrm{~T}$ & $\mathrm{~T}$ \\
\hline 0.7178693865818460 & & 919064901466228 & 0.282 & 3761575541894 & & $\mathrm{~T}$ & $\mathrm{~T}$ \\
\hline 0.3854043422815749 & $0 . \varepsilon$ & 459564696080051 & 0.501 & 584611095003 & 1 & $\mathrm{~T}$ & $\mathrm{~T}$ \\
\hline 0.2108396333215268 & & 651005523005935 & 0.277 & 976877106563 & 1 & $\mathrm{~T}$ & $\mathrm{~T}$ \\
\hline 0.2141559619677125 & & 649098133017333 & 0.725 & 112501595278 & 1 & $\mathrm{~T}$ & $\mathrm{~T}$ \\
\hline 0.2284041321530026 & & 391139444652197 & 0.27 & 909630245840 & $\mathrm{~T}$ & $\mathrm{~T}$ & $\mathrm{~T}$ \\
\hline 0.2324753124973142 & & 386388273506938 & 0.72 & 680240923074 & 1 & $\mathrm{~T}$ & $\mathrm{~T}$ \\
\hline 0.7708912020194205 & & 5664681526170645 & $0.71 \mathrm{~s}$ & 644893021613 & $\mathrm{~T}$ & $\mathrm{~T}$ & $\mathrm{~T}$ \\
\hline 0.7671703562760541 & & 5566623986051553 & 0.27 & 720837994066 & 1 & $\mathrm{~T}$ & 1 \\
\hline 0.0000000000000000 & & 000000000000000 & 0.000 & 0000000000000 & F & F & $F$ \\
\hline 0.5000000000000000 & & 000000000000000 & 0.000 & 0000000000000 & $\mathrm{~F}$ & $\mathrm{~F}$ & I \\
\hline 0.0000000000000000 & & 0000000000000000 & 0.500 & 0000000000000 & $\mathrm{~F}$ & F & $F$ \\
\hline 0.0137196548325491 & & 1679299773996945 & 0.994 & 3843582116898 & $\mathrm{~T}$ & $\mathrm{~T}$ & $\mathrm{~T}$ \\
\hline 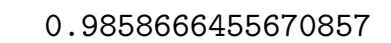 & & 9 & & . & $\mathrm{T}$ & $\mathrm{T}$ & \\
\hline
\end{tabular}

\section{KPOINTS}

The KPOINTS file specifies the size of the k-point mesh VASP is to use. VASP creates a system file, IBZKPT, which includes the list of all k-points. This can be done manually but normally it is completely sufficient to just use KPOINTS with automatic mesh generation, as follows:

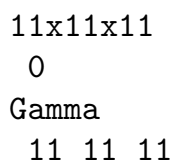


where

- the first line is a comment line;

- the second line is normally the number of k-points, a value of 0 triggers automatic k-point mesh generation;

- Gamma includes the $\Gamma$-point, the alternative is Monkhorst for MonkhorstPack k-point mesh. For odd numbers of k-points along each side the $\Gamma$-point is included in the Monkhorst-Pack mesh anyway;

- finally, the number of k-point divisions along each unit vector basis direction.

\section{POTCAR}

POTCAR files for each element are supplied with VASP, a POTCAR file for each run is constructed by concatenating the appropriate individual element files in the order that the positions are given in the POSCAR file.

A POTCAR file takes the following format (given for oxygen):

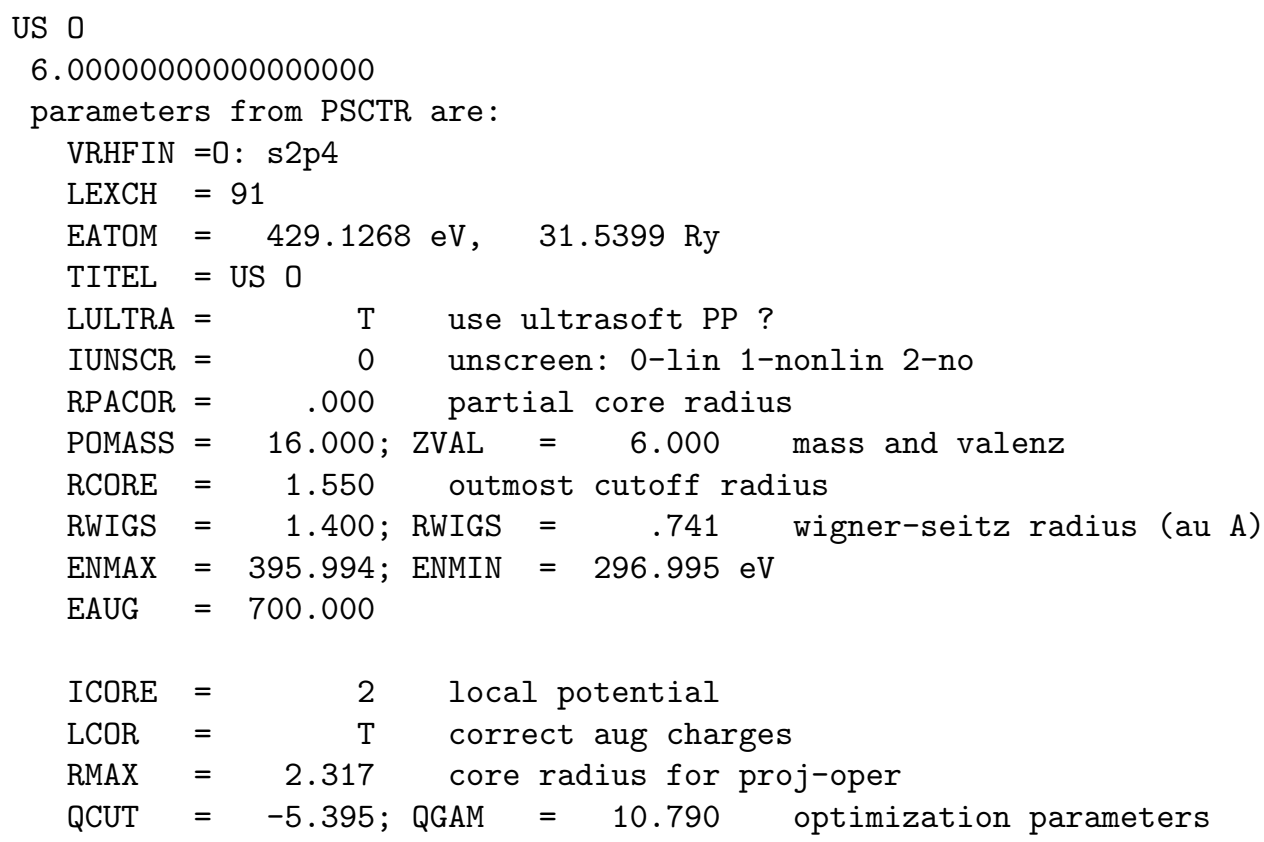




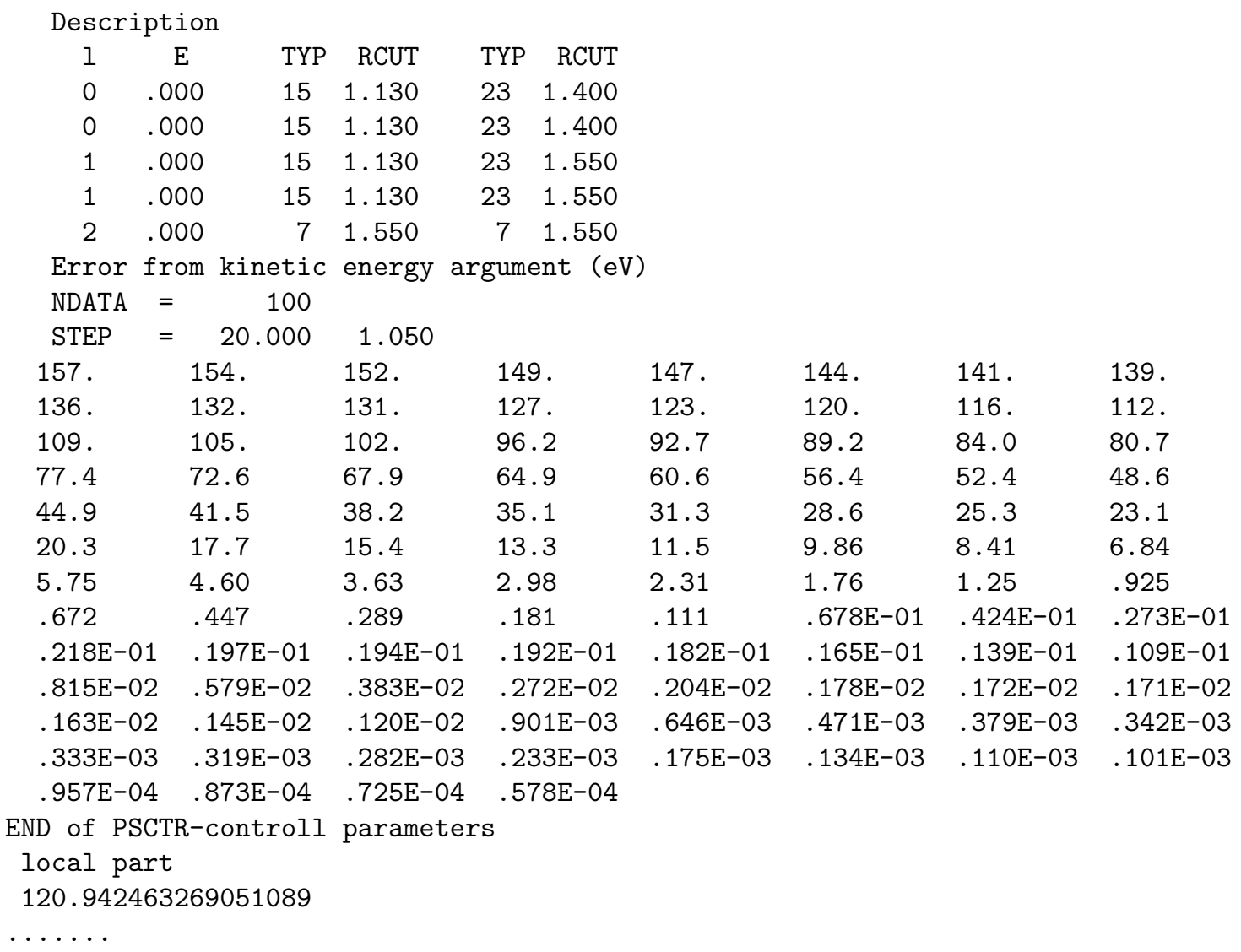

followed by the wavefunctions themselves, in both reciprocal and real-space co-ordinates. 


\section{INCAR}

The INCAR input file for the initial relaxation of a system in VASP would typically include the following parameters:

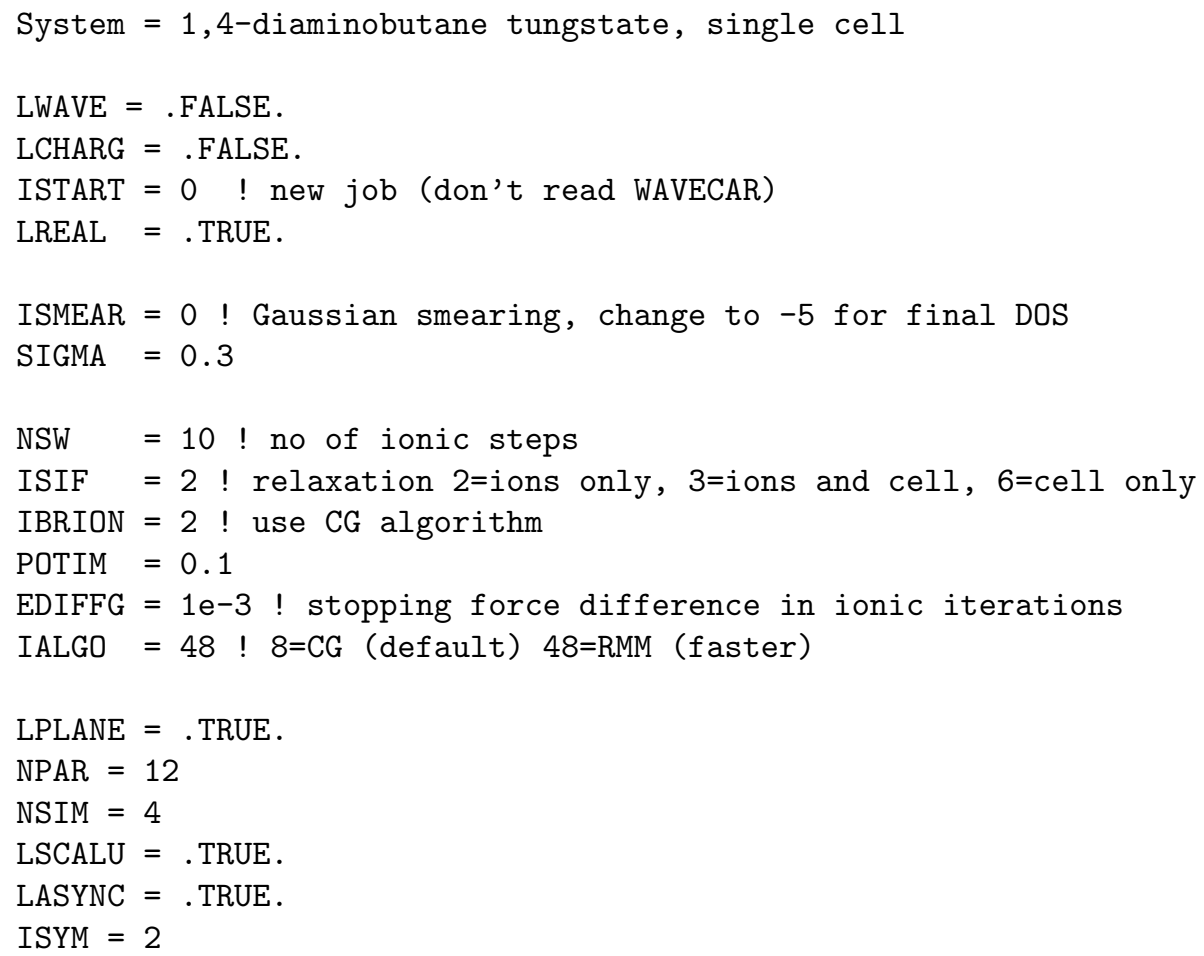

In the case above, the WAVECAR and CHGCAR files (wavefunctions and charge density) are very large and it is chosen not to write them to disk, and real space co-ordinates are used as the cell is too large to use reciprocal space. Gaussian smearing (commonly used for insulating and semiconducting systems) with a smearing parameter $\sigma$ of 0.3 is used. (For conducting systems, i.e. the tungsten bronzes, Fermi smearing (ISMEAR $=-1$ ) is used with $\sigma=0.15$.) Only the ions are relaxed, up to a maximum of 10 steps, using the conjugate-gradient algorithm. The electrons are relaxed using the residual minimisation method and are considered relaxed when the energy difference between electronic iterations is less than $1 \times 10^{-3} \mathrm{eV}$. The final six parameters are necessary for parallelisation, in this case on 12 processors.

While ISIF $=3$ is the most intuitive parameter to use as it allows both the ions and the lattice to relax, there are cases where it can cause the cal- 
culations to diverge. This was a problem particularly when calculating the hybrid systems and so relaxations using the above INCAR file with alternating ISIF $=2$ and ISIF $=6$ were performed, until each had converged.

Once relaxed, the following parameters would be changed to obtain an accurate ground state energy and partial density-of-states:

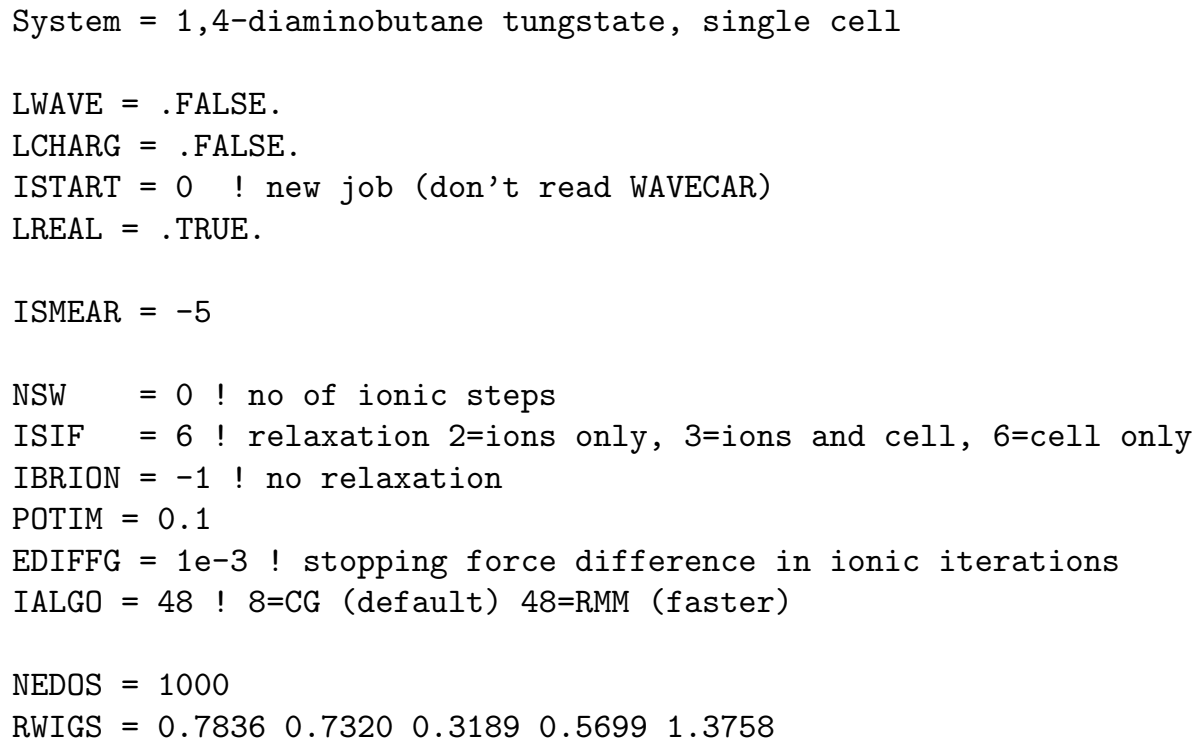

The changes from the INCAR files used for relaxation are summarised as follows:

- Smearing is changed from Fermi to tetrahedral to allow an accurate ground state energy to be calculated;

- NSW is set to zero and IBRION is set to -1 so no relaxation steps are permitted (as the tetrahedral smearing can sometimes cause divergent behaviour);

- The number of points in the density-of-states curve, and the WignerSeitz radius for each atomic species is listed (once again in the order the elements appear in the POTCAR file);

- Density-of-states calculations cannot be performed in parallelisation mode, so these parameters are removed. 


\section{Output}

Several files are created by VASP:

- CONTCAR gives the revised atomic positions and is of the same format as POSCAR, enabling the user to simply copy overwrite POSCAR with CONTCAR and continue the relaxation if required;

- WAVECAR, if written, contains the complete wavefunctions. This is useful to save time in repeated relaxation steps, however the WAVECAR files are very large;

- CHGCAR, if written, gives the charge density, with a more concise version in the $\mathrm{CHG}$ file;

- DOSCAR, if written, the density-of-states;

- OUTCAR gives the full details for each electronic and ionic iteration;

- OSZICAR gives the energy after each electronic and ionic step, similar to what one sees printed to the screen during a calculation. A sample OSZICAR file for a converged system is as follows:

\begin{tabular}{|c|c|c|c|c|c|c|c|}
\hline & $\mathrm{N}$ & $\mathrm{E}$ & $\mathrm{dE}$ & d eps & $\mathrm{ncg}$ & $\mathrm{rms}$ & $\operatorname{rms}(c)$ \\
\hline RMM : & 1 & $0.756552945980 \mathrm{E}+03$ & $0.75655 \mathrm{E}+03$ & $-0.22119 \mathrm{E}+04$ & 31968 & $0.941 \mathrm{E}+02$ & \\
\hline RMM : & 2 & $0.136813827545 \mathrm{E}+03$ & $-0.61974 \mathrm{E}+03$ & $-0.63448 E+03$ & 31968 & $0.288 \mathrm{E}+02$ & \\
\hline RMM : & 3 & $-0.310527341651 E+02$ & $-0.16787 E+03$ & $-0.16882 \mathrm{E}+03$ & 31968 & $0.201 \mathrm{E}+02$ & \\
\hline RMM : & 4 & $-0.110542140654 \mathrm{E}+03$ & $-0.79489 E+02$ & $-0.56728 \mathrm{E}+02$ & 31968 & $0.149 \mathrm{E}+02$ & \\
\hline RMM : & 5 & $-0.139088062063 E+03$ & $-0.28546 \mathrm{E}+02$ & $-0.25431 \mathrm{E}+02$ & 31968 & $0.107 \mathrm{E}+02$ & \\
\hline RMM : & 6 & $-0.153035069861 E+03$ & $-0.13947 \mathrm{E}+02$ & $-0.12814 E+02$ & 31968 & $0.647 \mathrm{E}+01$ & \\
\hline RMM : & 7 & $-0.159853589416 E+03$ & $-0.68185 E+01$ & $-0.63856 \mathrm{E}+01$ & 31968 & $0.495 \mathrm{E}+01$ & \\
\hline RMM : & 8 & $-0.163401649797 \mathrm{E}+03$ & $-0.35481 E+01$ & $-0.33365 \mathrm{E}+01$ & 31968 & $0.306 \mathrm{E}+01$ & \\
\hline RMM : & 9 & $-0.165222476168 \mathrm{E}+03$ & $-0.18208 E+01$ & $-0.17247 E+01$ & 31968 & $0.244 \mathrm{E}+01$ & \\
\hline RMM : & 10 & $-0.167318388587 \mathrm{E}+03$ & $-0.20959 E+01$ & $-0.20444 E+01$ & 97467 & $0.150 \mathrm{E}+01$ & \\
\hline RMM : & 11 & $-0.167332341079 \mathrm{E}+03$ & $-0.13952 \mathrm{E}-01$ & $-0.42603 \mathrm{E}-01$ & 91969 & $0.118 \mathrm{E}+00$ & \\
\hline RMM : & 12 & $-0.167333158382 \mathrm{E}+03$ & $-0.81730 \mathrm{E}-03$ & $-0.12712 \mathrm{E}-02$ & 74467 & $0.549 \mathrm{E}-01$ & $0.246 \mathrm{E}+01$ \\
\hline RMM : & 13 & $-0.179212345695 E+03$ & $-0.11879 E+02$ & $-0.27802 \mathrm{E}+02$ & 80148 & $0.647 \mathrm{E}+01$ & $0.364 E+01$ \\
\hline RMM : & 14 & $-0.150249746063 E+03$ & $0.28963 E+02$ & $-0.91688 \mathrm{E}+01$ & 72649 & $0.433 \mathrm{E}+01$ & $0.783 E+00$ \\
\hline RMM : & 15 & $-0.149080415141 E+03$ & $0.11693 \mathrm{E}+01$ & $-0.10802 \mathrm{E}+01$ & 80089 & $0.163 E+01$ & $0.345 \mathrm{E}+00$ \\
\hline RMM : & 16 & $-0.148865177182 \mathrm{E}+03$ & $0.21524 \mathrm{E}+00$ & $-0.12453 \mathrm{E}+00$ & 78017 & $0.621 \mathrm{E}+00$ & $0.148 \mathrm{E}+00$ \\
\hline RMM : & 17 & $-0.148828453652 \mathrm{E}+03$ & $0.36724 \mathrm{E}-01$ & $-0.33512 E-01$ & 83633 & $0.224 \mathrm{E}+00$ & $0.839 E-01$ \\
\hline RMM : & 18 & $-0.148819768147 \mathrm{E}+03$ & $0.86855 \mathrm{E}-02$ & $-0.46802 \mathrm{E}-02$ & 79536 & $0.113 \mathrm{E}+00$ & $0.293 E-01$ \\
\hline RMM : & 19 & $-0.148819684280 E+03$ & $0.83867 \mathrm{E}-04$ & $-0.13404 \mathrm{E}-02$ & 81100 & $0.487 \mathrm{E}-01$ & $0.206 \mathrm{E}-01$ \\
\hline RMM : & 20 & $-0.148818886306 \mathrm{E}+03$ & $0.79797 \mathrm{E}-03$ & $-0.25664 \mathrm{E}-03$ & 78255 & $0.235 \mathrm{E}-01$ & $0.436 \mathrm{E}-02$ \\
\hline RMM : & 21 & $-0.148818936651 E+03$ & $-0.50344 E-04$ & $-0.58843 \mathrm{E}-04$ & 64549 & $0.105 \mathrm{E}-01$ & \\
\hline
\end{tabular}

More details can be found in the VASP guide, Ref. [302]. 


\section{Bibliography}

[1] K. S. Ahn, Y. C. Nah, Y. E. Sung, K. Y. Cho, S. S. Shin and J. K. Park, Appl. Phys. Lett. 81 (2002) 3930.

[2] D. Altermatt and I. D. Brown, Acta Cryst. B 41 (1985) 240.

[3] M. Amberg, J. R. Günter, H. Schmalle and G. Blasse, J. Solid State Chem. 77 (1988) 162.

[4] M. H. Anderson, J. R. Ensher, M. R. Matthews, C. E. Wieman and E. A. Cornell, Science 269 (1995) 198.

[5] P. Anderson, The Theory of Superconductivity in the High- $T_{C}$ Cuprates (Princeton Unviersity Press, Princeton NJ, 1997).

[6] H. Arend, W. Huber, F. H. Mischgofsky and G. K. R. van Leeuwen, J. Cryst. Growth 43 (1978) 213.

[7] Y. Arimoto, S. I. Ohkoshi, Z. J. Zhong, H. Seino, Y. Mizobe and K. Hashimoto, J. Am. Chem. Soc. 125 (2003) 9240.

[8] E. M. Arnett, F. M. Jones, M. Taagepera, W. G. Henderson, J. L. Beauchamp, D. Holtz and R. W. Taft, J. Am. Chem. Soc. 94 (1972) 4724 .

[9] N. W. Ashcroft and N. D. Mermin, Solid State Physics (Saunders College Publishing, Philadelphia, 1976).

[10] D. H. Aue, H. M. Webb and M. T. Bowers, J. Am. Chem. Soc. 94 (1972) 4726. 
[11] P. Avouris, Chem. Phys. 281 (2002) 429.

[12] G. Aylward and T. Findlay, SI Chemical Data (Jacaranda Wiley Ltd., Brisbane, 1994).

[13] S. Ayyappan, G. N. Subbanna and C. N. R. Rao, Chem. Eur. J. 1 (1995) 165.

[14] J. C. Bailar, H. J. Emeleus, R. Nyholm and A. F. Trotman-Dickenson (Eds.). Comprehensive Inorganic Chemistry, vol. 4, chap. 50: Tungsten Bronzes, Vanadium Bronzes and Related Compounds (Pergamon, Oxford, 1973).

[15] J. C. Bailar, H. J. Emeleus, R. Nyholm and A. F. TrotmanDickenson (Eds.). Comprehensive Inorganic Chemistry, vol. 3, chap. 36: Chromium, Molybdenum and Tungsten, p. 623 (Pergamon, Oxford, 1973).

[16] C. Balázsi and J. Pfeifer, Solid State Ionics 124 (1999) 73.

[17] C. Balázsi and J. Pfeifer, Sol. En. Mat. Sol. Cells 76 (2003) 577.

[18] M. E. Baldwin, Spectrochim. Acta 18 (1962) 1455.

[19] K. Bange, Sol. En. Mat. Sol. Cells 58 (1999) 1.

[20] T. Barnes and J. Riera, Phys. Rev. B 50 (1994) 6817.

[21] E. M. Bauer, C. Bellitto, M. Colapietro, G. Portalone and G. Righini, Inorg. Chem. 42 (2003) 6345.

[22] P. Beaudot, M. E. D. Roy and J. P. Besse, Chem. Mater. 16 (2004) 935.

[23] P. Beaudot, M. E. D. Roy and J. P. Besse, J. Solid State Chem. 177 (2004) 2691.

[24] C. Bellitto, F. Federici, M. Colapietro, G. Portalone and D. Caschera, Inorg. Chem. 41 (2002) 709. 
[25] C. Bernhard, J. L. Tallon, C. Niedermayer, T. Blasius, A. Golnik, E.Brücher, R. K. Kremer, D. R. Noakes, C. E. Stronach and E. J. Ansaldo, Phys. Rev. B 59 (1999) 14099.

[26] A. Bessière, J. C. Badot, M. C. Certiat, J. Ligave, V. Lucas and N. Baffier, Electrochim. Acta 46 (2001) 2251.

[27] D. Bitauld, unpublished (internal report).

[28] F. Bloch, Z. Physik. 52 (1928) 555.

[29] J. Booth, T. Ekström, E. Iguchi and R. J. D. Tilley, J. Solid State Chem. 41 (1982) 293.

[30] J. I. Brauman, J. M. Riveros and L. K. Blair, J. Am. Chem. Soc. 93 (1971) 3914.

[31] E. O. Brimm, J. C. Brantley, J. H. Lorenz and M. H. Jellinek, J. Am. Chem. Soc. 73 (1951) 5427.

[32] B. W. Brown and E. Banks, Phys. Rev. 84 (1951) 609.

[33] B. W. Brown and E. Banks, J. Am. Chem. Soc. 76 (1954) 963.

[34] I. D. Brown and D. Altermatt, Acta Cryst. B 41 (1985) 244.

[35] D. W. Bullett, J. Phys. C 16 (1983) 2197.

[36] A. Butera, A. Fainstein, E. Winkler and J. Tallon, Phys. Rev. B 63 (2001) 054442.

[37] E. Cariati, D. Roberto, R. Ugo, P. Ford, S. Galli and A. Sironi, Chem. Mater 14 (2002) 5116.

[38] B. L. Chamberland, Inorg. Chem. 8 (1969) 1183.

[39] W. M. Chang and S. L. Wang, Chem. Mater. 17 (2005) 74.

[40] A. Chemseddine, R. Morineau and J. Livage, Solid State Ionics 9-10 (1983) 357. 
[41] R. Chen, P. Y. Zavalij, M. S. Whittingham, J. E. Greedan, N. P. Raju and M. Bieringer, J. Mater. Chem. 9 (1999) 93.

[42] W. Chen, F. Liu and X. You, J. Solid State Chem. 167 (2002) 119.

[43] W. Chen, H. M. Yan, J. Y. Wang, Z. Y. Liu, J. J. Xu, M. Yang and J. S. Chen, J. Am. Chem. Soc. 125 (2003) 9266.

[44] Z. Y. Cheng, B. X. Gao, M. L. Pang, S. Y. Wang, Y. C. Han and J. Lin, Chem. Mater. 15 (2003) 4705.

[45] A. M. Chippindale and A. D. Law, J. Solid State Chem. 142 (1999) 236.

[46] K. Chondroudis, D. B. Mitzi and P. Brock, Chem. Mater. 11 (1999) 3028.

[47] K. Chondroudis, D. B. Mitzi and P. Brock, Chem. Mater. 12 (2000) 169.

[48] S. V. Chong, B. Ingham and J. L. Tallon, Curr. Appl. Phys. 4 (2004) 197.

[49] S. V. Chong, B. Ingham and J. L. Tallon, Mat. Res. Soc. Symp. Proc. 847 (2005) E13.14.

[50] J. H. Choy, J. Phys. Chem. Solids 65 (2004) 373.

[51] J. H. Choy, Y. S. Han, N. G. Park, H. Kim and S. W. Kim, Synth. Met. 71 (1995) 2053.

[52] J. H. Choy, S. J. Hwang and N. G. Park, J. Am. Chem. Soc. 119 (1997) 1624.

[53] J. H. Choy, N. G. Park, S. J. Hwang and D. H. Kim, J. Am. Chem. Soc. 116 (1994) 11564.

[54] U. C. Chung, J. L. Mesa, J. L. Pizarro, L. Lezama, J. S. Garitaonandia, J. P. Chapman and M. I. Arriortua, J. Solid State Chem. 177 (2004) 2705 . 
[55] A. Clearfield, A. Moini and P. R. Rudolf, Inorg. Chem. 24 (1985) 4606.

[56] J. M. Clemente-Juan, E. Coronado, A. Forment-Aliaga, J. R. GalanMascaros, C. Gimenez-Saiz and C. J. Gomez-Garcia, Inorg. Chem. 43 (2004) 2689.

[57] F. Corà and C. R. A. Catlow, Phys. Stat. Sol. B 217 (2000) 577.

[58] F. Corà, M. G. Stachiotti and C. R. A. Catlow, J. Phys. Chem. B 101 (1997) 3945.

[59] A. B. Corradi, A. M. Ferrari, L. Righi and P. Sgarabotto, Inorg. Chem. 40 (2001) 218.

[60] F. A. Cotton and R. M. Wing, Inorg. Chem. 4 (1965) 867.

[61] N. B. Culthop, L. H. Daly and S. E. Wiberley, Introduction to Infrared and Raman Spectroscopy. 2nd ed. (Academic Press, New York, 1975).

[62] E. Dagotto, Rep. Prog. Phys. 62 (1999) 1525.

[63] E. Dagotto and T. M. Rice, Science 271 (1996) 618.

[64] E. Dagotto, J. Riera and D. Scalapino, Phys. Rev. B 45 (1992) 5744.

[65] Z. Dai, Q. Shi, G. Li, D. Zhang, W. Fu, H. Jin, W. Xu and S. Feng, Inorg. Chem. 42 (2003) 7396.

[66] M. F. Daniel, B. Desbat, J. C. Lassegues, B. Gerand and M. Figlarz, J. Solid State Chem. 67 (1987) 235.

[67] W. C. Dautremont-Smith, M. Green and K. S. Kang, Electrochim. Acta 22 (1977) 751.

[68] D. Davazoglou and A. Donnadieu, Thin Solid Films 147 (1987) 131.

[69] R. F. de Farias, Int. J. Inorg. Mater. 3 (2001) 303.

[70] R. F. de Farias and C. Airoldi, J. Phys. Chem. Solids 64 (2003) 2199. 
[71] L. J. de Jongh and A. R. Miedema, Adv. Phys. 23 (1974) 1.

[72] L. J. de Jongh, W. D. van Amstel and A. R. Miedema, Physica 58 (1972) 277.

[73] T. Devic, M. Evain, Y. Moelo, E. Canadell, P. Auban-Senzier, M. Fourmigue and P. Batail, J. Am. Chem. Soc. 125 (2003) 3295.

[74] R. Dingle, M. E. Lines and S. L. Holt, Phys. Rev. 187 (1969) 643.

[75] N. D. Draper, R. J. Batchelor, B. C. Sih, Z. G. Ye and D. B. Leznoff, Chem. Mater. 15 (2003) 1612.

[76] D. M. Edwards, Adv. Phys. 51 (2002) 1259.

[77] A. V. Ellis, J. D’Arcy-Gall, K. Vijayamohanan, R. Goswami, P. G. Ganesan, C. Ryu and G. Ramanath, Thermochim. Acta 426 (2005) 207.

[78] M. Era, T. Hattori, T. Taira and T. Tsutsui, Chem. Mater. 9 (1997) 8.

[79] M. Era, S. Morimoto, T. Tsutsui and S. Saito, Appl. Phys. Lett. 65 (1994) 676.

[80] I. Felner, U. Asaf, M. Weger, S. Reich and G. Leitus, Physica B 311 (2002) 191.

[81] S. Fernandez, J. L. Pizarro, J. L. Mesa, L. Lezama, M. I. Arriortua, R. Olazcuaga and T. Rojo, Inorg. Chem. 40 (2001) 3476.

[82] M. Figlarz, Prog. Solid State Chem. 19 (1989) 1.

[83] M. E. Fisher, Am. J. Phys. 32 (1964) 343.

[84] J. C. P. Gabriel, R. Nagarajan, S. Natarajan, A. K. Cheetham and C. N. R. Rao, J. Solid State Chem. 129 (197) 257.

[85] S. Gago, M. Pillinger, A. A. Valente, T. M. Santos, J. Rocha and I. S. Goncalves, Inorg. Chem. 43 (2004) 5422. 
[86] G. Gainsford, N. Robinson and J. Tallon, Acta Cryst. E 58 (2002) m521.

[87] G. J. Gainsford, S. V. Chong, B. Ingham and J. L. Tallon, Acta Cryst. E 58 (2002) 93.

[88] Q. Gao, O. Giraldo, W. Tong and S. L. Suib, Chem. Mater. 13 (2001) 778.

[89] W. R. Gardner and G. C. Danielson, Phys. Rev. 93 (1954) 46.

[90] J. K. Garland, K. Emerson and M. R. Presspich, Acta Cryst. C 46 (1990) 1603.

[91] C. Genin, A. Driouiche, B. Gerand and M. Figlarz, Solid State Ionics 53 (1992) 315.

[92] A. Georg, W. Graf and V. Wittwer, Sol. En. Mat. Sol. Cells 51 (1998) 353.

[93] K. Gesheva, A. Szekeres and T. Ivanova, Sol. En. Mat. Sol. Cells 76 (2003) 563.

[94] O. Glemser and C. Naumann, Z. anorg. Chem. 265 (1951) 288.

[95] O. Glemser and H. Sauer, Z. anorg. Chem. 252 (1943) 144.

[96] R. B. Goldner, T. E. Haas, G. Seward, K. K. Wong, P. Norton, G. Foley, G. Berera, G. Wei, S. Schulz and R. Chapman, Solid State Ionics 2830 (1998) 1715.

[97] V. M. Goldschmidt, Naturwiss. 14 (1926) 477.

[98] P. Gomez-Romero, Adv. Mater. 13 (2001) 163.

[99] J. Gopalakrishnan, N. S. P. Bhuvanesh and A. R. Raju, Chem. Mater. 6 (1994) 373.

[100] C. G. Granqvist, Handbook of Inorganic Electrochromic Materials (Elsevier, Amsterdam, 1995). 
[101] C. G. Granqvist, Electrochim. Acta 44 (1999) 3005.

[102] C. Grenthe and M. Sundberg, J. Solid State Chem. 167 (2002) 402.

[103] J. Guan, Z. Tang and A. M. Guloy, Chem. Commun. p. 1833.

[104] J. R. Günter and E. Dubler, J. Solid State Chem. 65 (1986) 118.

[105] J. R. Günter, H. W. Schmalle and E. Dubler, Solid State Ionics 43 (1990) 85.

[106] J. D. Guo, K. P. Reis and M. S. Whittingham, Solid State Ionics 53 (1992) 315.

[107] J. R. Gutnick, E. A. Muller, A. N. Sarjeant and A. J. Norquist, Inorg. Chem. 43 (2004) 6528.

[108] G. Guzman, B. Yebka, J. Livage and C. Julien, Solid State Ionics 86-88 (1996) 407.

[109] P. J. Hagrman and J. Zubieta, Inorg. Chem. 40 (2001) 2800.

[110] S. Halasyamani, K. R. Heier, C. L. Stern and K. R. Poeppelmeier, Acta Cryst. C 53 (1997) 1240.

[111] M. A. Halcrow, Dalton Trans. p. 4375.

[112] Z. Han, Y. Zhao, J. Peng, H. Ma, Q. Liu, E. Wang and N. Hu, J. Solid State Chem. 177 (2004) 4325.

[113] S. K. Haydon and D. A. Jefferson, J. Solid State Chem. 168 (2002) 306.

[114] A. Hjelm, C. G. Granqvist and J. M. Wills, Phys. Rev. B 54 (1996) 2436.

[115] B. S. Hobbs and A. C. C. Tseung, J. Electrochem. Soc. 119 (1972) 580.

[116] P. Hohenberg and W. Kohn, Phys. Rev. 136 (1964) 864B. 
[117] C. Hornick, P. Rabu and M. Drillon, Polyhedron 19 (2000) 259.

[118] R. Hott, R. Kleiner, T. Wolf and G. Zwicknagl, cond-mat/0408212 (2004).

[119] Z. L. Huang, M. Drillon, N. Masciocchi, A. Sironi, J. T. Zhao, P. Rabu and P. Panissod, Chem. Mater. 12 (2000) 2805.

[120] B. Ingham, S. V. Chong and J. L. Tallon, Mat. Res. Soc. Symp. Proc. 775 (2003) 165.

[121] B. Ingham, S. V. Chong and J. L. Tallon, Curr. Appl. Phys. 4 (2004) 202.

[122] A. J. Jacobson, J. W. Johnson and J. L. Lewandowski, Inorg. Chem. 24 (1985) 3727.

[123] H. A. Jahn and E. Teller, Proc. Roy. Soc. London A 161 (1937) 220.

[124] G. G. Janauer, A. Dobley, J. Guo, P. Y. Zavalij and M. Whittingham, Chem. Mater. 8 (1996) 2096.

[125] J. W. Johnson, A. J. Jacobson, S. M. Rich and J. F. Brody, J. Am. Chem. Soc. 103 (1981) 5246.

[126] D. C. Johnston, M. Troyer, S. Miyahara, D. Lidsky, K. Ueda, M. Azuma, Z. Hiroi, M. Takano, M. Isobe, Y. Ueda, M. Korotin, V. I. Anisimov, A. V. Mahajan and L. L. Miller, cond-mat/0001147 (2000).

[127] P. Judeinstein and J. Livage, Mater. Sci. Engineering B3 (1989) 129.

[128] P. Judeinstein, R. Morineau and J. Livage, Solid State Ionics 51 (1992) 239.

[129] C. R. Kagan, T. L. Breen and L. L. Kosbar, Appl. Phys. Lett. 79 (2001) 3536.

[130] C. R. Kagan, D. B. Mitzi and C. D. Dimitrakopolous, Science 286 (1999) 945. 
[131] A. I. Kahn and D. O'Hare, J. Mater. Chem. 12 (2002) 3191.

[132] H. Kamal, A. A. Akl and K. Abdel-Hady, Physica B 349 (2004) 192.

[133] R. O. Keeling Jr., Acta Cryst. 10 (1957) 209.

[134] D. L. Kepert, Prog. Inorg. Chem. 4 (1962) 199.

[135] M. I. Khan, E. Yohannes, R. C. Nome, S. Ayesh, V. O. Golub, C. J. O'Connor and R. J. Doedens, Chem. Mater. 16 (2004) 5273.

[136] O. Y. Khyzhun, Y. M. Solonin and V. D. Dobrovolsky, J. Alloys Compounds $\mathbf{3 2 0}$ (2001) 1.

[137] J. L. Kissick, A. R. Cowley and A. M. Chippindale, J. Solid State Chem. 167 (2002) 17.

[138] C. Kittel, Introduction to Solid State Physics, 7th ed. (John Wiley \& Sons, Inc., New York, 1996).

[139] H. Klauk, Phys. World 1 (2000) 18.

[140] W. Kohn and L. J. Sham, Phys. Rev. 140 (1965) 1133A.

[141] J. Kondo, Prog. Theor. Phys. 32 (1964) 37.

[142] J. Kondo, Solid State Phys. 23 (1969) 1.

[143] U. Kortz, N. K. Al-Kassem, M. G. Savelieff, N. A. A. Kadi and M. Sadakane, Inorg. Chem. 40 (2001) 4742.

[144] U. Kortz, S. Nellutla, A. C. Stowe, N. S. Dalal, U. Rauwald, W. Danquah and D. Ravot, Inorg. Chem. 43 (2004) 2308.

[145] B. Krebs, Acta Cryst. B 28 (1972) 2222.

[146] G. Kresse, Ph.D. thesis (Technische Univerität Wien) (1993).

[147] G. Kresse and J. Furthmüller, Comput. Mater. Sci. 6 (1996) 15.

[148] G. Kresse and J. Furthmüller, Phys. Rev. B 53 (1996) 11169. 
[149] G. Kresse and J. Hafner, Phys. Rev. B 47 (1993) RC558.

[150] G. Kresse and J. Hafner, J. Phys.: Condens. Matter 6 (1994) 8245.

[151] S. Krustev, K. Ivanov and D. Klissurski, J. Alloys Comp. 182 (1992) 189.

[152] M. Kudo, H. Ohkawa, W. Sugimoto, N. Kumada, Z. Liu, O. Terasaki and Y. Sugahara, Inorg. Chem. 42 (2003) 4479.

[153] M. Kurmoo, H. Kumagai, S. M. Hughes and C. J. Kepert, Inorg. Chem. 42 (2003) 6709.

[154] R. M. Kusters, J. Singleton, D. A. Keen, R. McGreevy and W. Hayes, Physica B 155 (1989) 362.

[155] S. J. Kwon and J. H. Choy, Inorg. Chem. 42 (2003) 8134.

[156] C. M. Lampert, Sol. En. Mat. Sol. Cells 52 (1998) 207.

[157] W. Lasocha, J. Jansen and H. Schenk, J. Solid State Chem. 117 (1995) 103.

[158] G. Lautenschläger, H. Weitzel, T. Vogt, R. Hock, A. Boehm, M. Bonnet and H. Füss, Phys. Rev. B 48 (1993) 6087.

[159] S. H. Lee, H. M. Cheong, C. E. Tracy, A. Mascarenhas, D. K. Benson and S. K. Deb, Electrochim. Acta 44 (1999) 3111.

[160] S. H. Lee, H. M. Cheong, C. E. Tracy, A. Mascarenhas, A. W. Czanderna and S. K. Deb, Appl. Phys. Lett. 75 (1999) 1541.

[161] S. H. Lee, H. M. Cheong, C. E. Tracy, A. Mascarenhas, J. R. Pitts, G. Jorgensen and S. K. Deb, Appl. Phys. Lett. 76 (2000) 3908.

[162] S. H. Lee, H. M. Cheong, J. G. Zhang, A. Mascarenhas, D. K. Benson and S. K. Deb, Appl. Phys. Lett. 74 (1999) 242.

[163] I. Lefkowitz, M. B. Dowell and M. A. Shields, J. Solid State Chem. 15 (1975) 24. 
[164] G. Leftheriotis, S. Papaefthimiou and P. Yianoulis, Sol. En. Mater. Sol. Cells 83 (2004) 115.

[165] Z. A. D. Lethbridge, M. J. Smith, S. K. Tiwary, A. Harrison and P. Lightfoot, Inorg. Chem. 43 (2004) 11.

[166] A. B. P. Lever, Inorganic Electronic Spectroscopy, Studies in Physical and Theoretical Chemistry, vol. 33, 2nd ed. (Elsevier Science Publishers, Amsterdam, 1984).

[167] Y. Levi, O. Millo, A. Sharoni, Y. Tsabba, G. Leitus and S. Reich, Europhys. Lett. 51 (2000) 564.

[168] C. Li, G. Wang, D. G. Evans and X. Duan, J. Solid State Chem. 177 (2004) 4569.

[169] G. Li, Z. Shi, X. Liu, Z. Dai, L. Gao and S. Feng, Inorg. Chem. 43 (2004) 8224.

[170] K. C. Li and C. Y. Wang, Tungsten. 2nd ed. (Reinhold Publishing Corporation, New York, 1947).

[171] K. Liang, D. B. Mitzi and M. T. Prikas, Chem. Mater. 10 (1998) 403.

[172] A. A. Likalter, Physica B 315 (2002) 252.

[173] Z. E. Lin, J. Zhang, Y. Q. Sun and G. Y. Yang, Inorg. Chem. 43 (2004) 797.

[174] D. R. Linde (Ed.), CRC Handbook of Chemistry and Physics, 81st ed. (CRC Press, Boco Raton, 2000).

[175] Z. Liu, K. Ooi, H. Kanoh, W. Tang and T. Tomida, Langmuir 16 (2000) 4154.

[176] Z. Liu, L. Weng, Z. Chen and D. Zhao, Inorg. Chem. 42 (2003) 5960.

[177] J. Livage and G. Guzman, Solid State Ionics 84 (1996) 205. 
[178] A. J. Locock and P. C. Burns, J. Solid State Chem. 177 (2004) 2675.

[179] T. Loiseau, H. Muguerra, G. Férey, M. Haouas and F. Taulelle, J. Solid State Chem. 178 (2005) 621.

[180] G. S. Long, M. Wei and R. D. Willett, J. Am. Chem. Soc. 110 (1988) 8639.

[181] J. Lu, E. Shen, M. Yuan, Y. Li, E. Wang, C. Hu, L. Xu and J. Peng, Inorg. Chem. 42 (2003) 6956.

[182] Y. Lu, E. Wang, J. Chen, Y. Qi, C. Hu, L. Xu and J. Peng, J. Solid State Chem. 177 (2004) 946.

[183] Y. Lu, Y. Xu, E. Wang, Y. Li, L. Wang, C. Hu and L. Xu, J. Solid State Chem. 177 (2004) 2210.

[184] J. Lue, Y. Li, E. Shen, M. Yuan, E. Wang, C. Hu and L. Xu, J. Solid State Chem. 177 (2004) 1771.

[185] J. W. Lynn, B. Keimer, C. Ulrich, C. Bernhard and J. L. Tallon, Phys. Rev. B 61 (2000) R14964.

[186] H. Machiyama, Y. Kurihara and T. Azetsu, J. Korean Phys. Soc. 32 (1998) 156.

[187] A. Magneli, Ark. Kemi 1 (1949) 223.

[188] A. Magneli, Ark. Kemi 1 (1950) 513.

[189] S. Mandal, G. Kavitha, C. Narayana and S. Natarajan, J. Solid State Chem. 177 (2004) 2198.

[190] J. D. Martin, R. F. Hess and P. D. Boyle, Inorg. Chem. 43 (2004) 3242.

[191] N. D. Mathur, F. M. Grosche, S. R. Julian, I. R. Walker, D. M. Freye, R. K. W. Haselwimmer and G. G. Lonzarich, Nature 394 (1998) 39. 
[192] M. McElfresh, Fundamentals of Magnetism and Magnetic Measurements, published by Quantum Design, available online at http://www.qdusa.com/resources/tdrequestsmain.html.

[193] N. Mercier, S. Poiroux, A. Riou and P. Batail, Inorg. Chem. 43 (2004) 8361.

[194] A. J. Millis, P. B. Littlewood and B. I. Shraiman, Phys. Rev. Lett. 74 (1995) 5144.

[195] D. B. Mitzi, Chem. Mater. 8 (1996) 791.

[196] D. B. Mitzi, Prog. Inorg. Chem. 48 (1999) 1.

[197] D. B. Mitzi, Inorg. Chem. 39 (2000) 6107.

[198] D. B. Mitzi and P. Brock, Inorg. Chem. 40 (2001) 2096.

[199] D. B. Mitzi, C. D. Dimitrakopoulos, J. Rosner, D. R. Medeiros, Z. Xu and C. Noyan, Adv. Mater. 14 (2002) 1772.

[200] D. B. Mitzi, C. A. Field, W. T. A. Harrison and A. M. Guloy, Nature 369 (1994) 467.

[201] D. B. Mitzi, C. A. Field, Z. Schlesinger and R. B. Laibowitz, J. Solid State Chem. 114 (1995) 159.

[202] D. B. Mitzi and K. Liang, Chem. Mater. 9 (1997) 2990.

[203] D. B. Mitzi and K. Liang, J. Solid State Chem. 134 (1997) 376.

[204] D. B. Mitzi, D. R. Medeiros and P. R. L. Malenfant, Inorg. Chem. 41 (2002) 2134.

[205] D. B. Mitzi, M. T. Prikas and K. Chondroudis, Chem. Mater. 11 (1999) 542 .

[206] M. F. Mostafa and R. D. Willett, Phys. Rev. B 4 (1971) 2213. 
[207] B. Mourey, M. Hareng, B. Dumont, J. Desseine and M. Figlarz, Eurodisplay Proc. p. 223.

[208] J. A. Mydosh, Spin Glasses: An Experimental Introduction (Taylor \& Francis Ltd., London, 1993).

[209] J. Nagai, Electrochim. Acta 46 (2001) 2049.

[210] K. Ohhashi, T. Horiuchi, S. Nasu and E. Sugimata, J. Phys. Chem. 65 (2004) 603.

[211] Y. Omomo, T. Sasaki, L. Wang and M. Watanabe, J. Am. Chem. Soc. 125 (2003) 3568.

[212] B. Orel, U. O. Krašovec, U. L. Štangar and P. Judeinstein, J. Sol-Gel Sci. Tech. 11 (1998) 87.

[213] W. Orellana, A. J. R. da Silva and A. Fazzio, Phys. Rev. Lett. 87 (2001) 155901.

[214] H. Ott, H. Rudinger, Z. Fisk and J. L. Smith, Phys. Rev. Lett. 50 (1983) 1595.

[215] L. Ottaviano, F. Bussolotti, L. Lozzi, M. Passacantando, S. L. Rosa and S. Santucci, Thin Solid Films 436 (2003) 9.

[216] I. Pabst, H. Füss and J. W. Bats, Acta Cryst. C 43 (1987) 413.

[217] C. Panagopoulos, A. P. Petrovic, A. D. Hillier, J. L. Tallon, C. A. Scott and B. D. Rainford, Phys. Rev. B 69 (2004) 144510.

[218] H. Park, R. Lam, J. Greedan and J. Barbier, Chem. Mater. 15 (2003) 1703.

[219] E. Paterson, Am. Mineral. 66 (1981) 424.

[220] J. P. Perdew, K. Burke and M. Ernzerhof, Phys. Rev. Lett. 77 (1996) 3865 . 
[221] L. Pitaevskii and S. Stringari, Bose-Einstein Condensation, International Series of Monographs on Physics (Oxford University Press, 2003).

[222] V. L. Pokrovsky, J. Mag. Mag. Mater. 200 (1999) 515.

[223] M. Požek, A. Dulčić, D. Paar, A. Hamzić, M. Basletić, E. Tafra, G. V. M. Williams and S. Krämer, Phys. Rev. B 65 (2002) 174514.

[224] W. Qu, W. Wlodarski and J. U. Meyer, Sens. Actuators B 64 (2000) 76.

[225] F. D. Quarto, A. D. Paola and C. Sunseri, Electrochim. Acta 26 (1981) 1177.

[226] N. Rajic, N. Logar, G. Mali and V. Kaucic, Chem. Mater. 15 (2003) 1734.

[227] A. P. Ramirez, J. Phys.: Condens. Matter 9 (1997) 8171.

[228] C. N. R. Rao, E. V. Sampathkumaran, R. Nagarajan, G. Paul, J. N. Behera and A. Choudhury, Chem. Mater. 16 (2004) 1441.

[229] R. S. Rarig Jr., R. Lam, P. Zavalij, J. K. Ngala, R. L. LaDuca Jr., J. E. Greedan and J. Zubieta, Inorg. Chem. 41 (2002) 2124.

[230] R. S. Rarig Jr. and J. Zubieta, J. Solid State Chem. 167 (2002) 370.

[231] H. Rebbah, M. Borel and B. Raveua, Mat. Res. Bull. 15 (1980) 317.

[232] H. Remy and G. Laves, Ber. Deut. Ges. 66 (1933) 401.

[233] G. D. Rieck, Tungsten and its Compounds (Pergamon, Oxford, 1967).

[234] R. S. Roth and J. L. Waring, J. Res. Natl. Bur. Stand. A 70 (1966) 281.

[235] A. Sabatini and S. Califano, Spectrochim. Acta 16 (1960) 677. 
[236] T. Sasagawa, P. K. Mang, O. P. Vajk, A. Kapitulnik and M. Greven, Phys. Rev. B 66 (2002) 184512.

[237] P. Schiffer, A. P. Ramirez, W. Bao and S. W. Cheong, Phys. Rev. Lett. 75 (1995) 3336.

[238] C. W. Searle and S. T. Wang, Can. J. Phys. 48 (1970) 2023.

[239] F. Sediri, N. Etteyeb, N. Steunou, C. Guyard-Duhayon, J. Maquet, N. Gharbi and J. Livage, J. Solid State Chem. 167 (2002) 407.

[240] L. Segal, Appl. Spec. 17 (1963) 21.

[241] L. Segal and F. V. Eggerton, Appl. Spec. 15 (1961) 112.

[242] A. Seguatni, M. Fakhfakh, M. Vauley and N. Jouini, J. Solid State Chem. 177 (2004) 3402.

[243] H. R. Shanks, Solid State Comm. 15 (1974) 753.

[244] V. Shivaiah, M. Nagaraju and S. K. Das, Inorg. Chem. 42 (2003) 6604.

[245] R. M. Silverstein, G. C. Basser and T. C. Morrill, Spectrometric Identification of Organic Compounds, 5th ed. (Wiley, New York, 1991).

[246] S. Skaarup and R. W. Berg, J. Solid State Chem. 26 (1978) 59.

[247] J. L. Song, J. G. Mao, Y. Q. Sun, H. Y. Zeng, R. K. Kremer and A. Clearfield, J. Solid State Chem. 177 (2004) 633.

[248] J. L. Song, H. H. Zhao, J. G. Mao and K. R. Dunbar, Chem. Mater. 16 (2004) 1884.

[249] Y. Song, P. Zavalij, N. A. Chernova, M. Suzuki and M. S. Whittingham, J. Solid State Chem. 175 (2003) 63.

[250] Y. Song, P. Y. Zavalij, N. A. Chernova and M. S. Whittingham, Chem. Mater. 15 (2003) 4968. 
[251] C. M. Soukoulis, K. Levin and G. S. Grest, Phys. Rev. Lett. 48 (1982) 1756.

[252] F. Steglich, J. Aarts, C. D. Bredl, W. Lieke, D. Meschede, W. Franz and H. Schäfer, Phys. Rev. Lett. 43 (1979) 1892.

[253] G. R. Stewart, Z. Fisk, J. O. Willis and J. L. Smith, Phys. Rev. Lett. 52 (1984) 679.

[254] U. Stollenwerk, Prax. Natur. (Chemie) 26 (1977) 34.

[255] M. E. Straumanis, J. Am. Chem. Soc. 71 (1949) 679.

[256] M. Sun, N. Xu, Y. W. Cao, J. N. Yao and E. G. Wang, J. Mater. Res. 15 (2000) 927.

[257] J. T. Szymanski and A. C. Roberts, Can. Mineral. 22 (1984) 681.

[258] M. F. Tang and K. H. Lii, J. Solid State Chem. 177 (2004) 1912.

[259] M. Tinkham, Introduction to Superconductivity, 2nd ed. (McGraw-Hill, 1996). Chapter 9.

[260] T. Tsumura and M. Inagaki, Solid State Ionics 104 (1997) 183.

[261] Y. Tsunoda, W. Sugimoto and Y. Sugahara, Chem. Mater. 15 (2003) 632.

[262] R. Ueda and J. Kobayashi, Phys. Rev. 91 (1953) 1565.

[263] P. Valequeiro, A. M. Chippindale, A. R. Cowley and A. V. Powell, Inorg. Chem. 42 (2003) 7846.

[264] W. D. van Amstel and L. J. de Jongh, Solid State Comm. 11 (1972) 1423.

[265] N. van der Post, F. L. Mettes, J. A. Mydosh, J. M. van Ruitenbeek and I. K. Yanson, Phys. Rev. B 53 (1996) R476. 
[266] L. G. van Uittert, R. C. Sherwood, H. J. Williams, J. J. Rubin and W. A. Bonner, J. Phys. Chem. Solids 25 (1964) 1447.

[267] D. Vanderbilt, Phys. Rev. B 41 (1990) 7892.

[268] C. Vaysse, L. Guerlou-Demourgues, E. Duguet and C. Delmas, Inorg. Chem. 42 (2003) 4559.

[269] K. von Klitzing, G. Dorda and M. Pepper, Phys. Rev. Lett. 45 (1980) 494.

[270] P. Y. Vuillaume, K. Glinel, A. M. Jonas and A. Laschewsky, Chem. Mater. 15 (2003) 3625.

[271] A. D. Walkingshaw, N. A. Spaldin and E. Artacho, Phys. Rev. B. 70 (2004) 165110.

[272] C. M. Wang, C. H. Liao, H. M. Lin and K. H. Lii, Inorg. Chem. 43 (2004) 8239.

[273] K. Z. Wang and L. H. Gao, Mat. Res. Bull. 37 (2002) 2447.

[274] L. Wang, Y. Ebina, K. Takada, K. Kurashima and T. Sasaki, Adv. Mater. 16 (2004) 1412.

[275] S. Wang, D. B. Mitzi, C. A. Field and A. M. Guloy, J. Am. Chem. Soc. 117 (1995) 5297.

[276] W. Wang, L. Xu, Y. Wei, F. Li, G. Gao and E. Wang, J. Solid State Chem. 178 (2005) 608.

[277] X. Wang, L. Liu and A. J. Jacobson, J. Solid State Chem. 177 (2004) 194.

[278] B. C. Watson, V. N. Kotov, M. W. Meisel, D. W. Hall, G. E. Granroth, W. T. Montfooij, S. E. Nagler, D. A. Jensen, R. Backov, M. A. Petruska, G. E. Fanucci and D. R. Talham, Phys. Rev. Lett. 86 (2001) 5168 . 
[279] D. Weber, Z. Naturforsch. 33 (1978) 1443.

[280] C. T. White and T. N. Todorov, Nature 393 (1998) 240.

[281] R. D. Willett, C. Galeriu, C. P. Landee, M. M. Turnbull and B. Twamley, Inorg. Chem. 43 (2004) 3804.

[282] R. D. Willett and K. R. Maxcy, Inorg. Chem. 41 (2002) 7024.

[283] R. D. Willett, H. Place and M. Middleton, J. Am. Chem. Soc. 110 (1988) 8639.

[284] G. V. M. Williams, A. C. McLaughlin, J. P. Attfield, S. Krämer and H. K. Lee, cond-mat/0108521 (2001).

[285] F. Wohler, Ann. Chim. Phys. 29 (1823) 43.

[286] W. P. Wolf, Braz. J. Phys. 30 (2000) 794.

[287] E. Wortham, A. Zorko, D. Arcon and A. Lappas, Physica B 318 (2002) 387.

[288] L. Xu, C. Qin, X. Wang, Y. Wei and E. Wang, Inorg. Chem. 42 (2003) 7342.

[289] Z. Xu and D. B. Mitzi, Inorg. Chem. 42 (2003) 6589.

[290] Z. Xu and D. B. Mitzi, Chem. Mater. 15 (2003) 3632.

[291] Z. Xu, D. B. Mitzi, C. D. Dimitrakopoulos and K. R. Maxcy, Inorg. Chem. 42 (2003) 2031.

[292] Z. Xu, D. B. Mitzi and D. R. Medeiros, Inorg. Chem. 42 (2003) 1400.

[293] H. C. Yao, Y. Z. Li, S. Gao, Y. Song and L. M. Zheng, J. Solid State Chem. 177 (2004) 4557.

[294] X. Y. Yi and L. M. Zheng, J. Solid State Chem. 168 (2002) 263.

[295] S. K. Yun and J. Maier, Chem. Mater. 11 (1999) 1644. 
[296] L. Zhang, Y. Wei, C. Wang, H. Guo and P. Wang, J. Solid State Chem. 177 (2004) 3433.

[297] Q. Zhang, C. Lu, W. Yang, S. Chen, Y. Yu, X. He, Y. Yan, J. Liu, X. Xu, C. Xia, X. Wu and L. Chen, J. Solid State Chem. 177 (2004) 2862.

[298] X. H. Zhu, N. Mercier, M. Allain, P. Frere, P. Blanchard, J. Roncali and A. Riou, J. Solid State Chem. 177 (2004) 1067.

[299] X. H. Zhu, N. Mercier, P. Frere, P. Blanchard, J. Roncali, M. Allain, C. Pasquier and A. Riou, Inorg. Chem. 42 (2003) 5330.

[300] Z.Yi, Y. Yang, K. Huang, G. Li, C. Chen, W. Wang, Y. Liu and W. Pang, J. Solid State Chem. 177 (2004) 4073.

[301] http://neon.otago.ac.nz/chemistry/chemsearch/micro.html.

[302] VASP guide available from http://cms.mpi.univie.ac.at/vasp/. 